Portland State University

PDXScholar

Summer 8-12-2013

\title{
Ecomorphology and Mating Behavior of Two Species of Night-stalking Tiger Beetles, Omus audouini and O. dejeanii
}

Robert Kent Richardson

Portland State University

Follow this and additional works at: https://pdxscholar.library.pdx.edu/open_access_etds

Part of the Other Animal Sciences Commons, and the Terrestrial and Aquatic Ecology Commons Let us know how access to this document benefits you.

\section{Recommended Citation}

Richardson, Robert Kent, "Ecomorphology and Mating Behavior of Two Species of Night-stalking Tiger Beetles, Omus audouini and O. dejeanii" (2013). Dissertations and Theses. Paper 1021.

https://doi.org/10.15760/etd.1021

This Dissertation is brought to you for free and open access. It has been accepted for inclusion in Dissertations and Theses by an authorized administrator of PDXScholar. Please contact us if we can make this document more accessible: pdxscholar@pdx.edu. 
Ecomorphology and Mating Behavior of Two Species of Night-stalking Tiger Beetles, Omus audouini and O. dejeanii

by

Robert Kent Richardson

A dissertation submitted in partial fulfillment of the requirements for the degree of

Doctor of Philosophy

in

Biology

Dissertation Committee:

Luis Ruedas, Chair

Catherine deRivera

Deborah Duffield

Michael Murphy

Randy Zelick

Portland State University

2013 
(C) 2013 Robert Kent Richardson 


\section{$\underline{\text { Abstract }}$}

Night-stalking tiger beetles (Cicindelinae: Omus) are among the least studied members of the highly diverse Carabid sub-family Cicindelinae, the tiger beetles. Despite populations of Omus being common in the forest floor habitats of the west coast of North America and their conspicuous predatory role within terrestrial arthropod communities, little is known about the biology and ecology of Omus.

Field studies showed that two species of Omus existed in the forested areas of Powell Butte Nature Park, Portland, Oregon, USA: Omus audouini and O. dejeanii. The co-occurrence of sympatric, and likely syntopic, species allowed for a comparative approach in examining and analyzing previously unknown or unaddressed aspects of the biology of Omus. Both morphometric and behavioral analysis was used to address specific questions regarding niche partitioning and mating behaviors in the genus.

On the basis of the competitive exclusion principle, I predicted that these closely related species with similar ecological requirements would experience selective pressure to minimize niche overlap and competitive pressures through morphological character displacement. In particular, the mandibles of male tiger beetles serve a dual role: one as tools for feeding — including prey capture and prey processing — and another role as secondary sexual organs whereby the males use their mandibles to grasp the female and maintain amplexus. A geometric morphometric approach was used to evaluate and compare shape differences between the two species as well as identify trends of sexual dimorphism and species differences in context of prey base.

Tiger beetles obligatorily engage in male-superior mounted mating behavior. Body size was used to first address trends of female-biased sexual size dimorphism 
within the Carabid subfamily Cicindelinae. Female tiger beetles may be expected to experience proportionally greater stress during mating among larger bodied than smaller bodied species and selection would favor increasingly pronounced female-biased sexual size dimorphism among larger-bodied species.

The mating duration of Omus was anecdotally reported as an order of magnitude greater than any other tiger beetle but has never been experimentally confirmed. I performed a series of pairings under laboratory setting to (1) establish a baseline of mating duration for the two species and test the effects of (2) time of day mating was initiated, (3) food deprivation and (4) operational sex ratio on mating duration.

Morphometric analysis suggested niche partitioning existed between the two species due to an average body size scaling factor of x 1.3 and an average mandible length scaling factor of x1.5, i.e. "Hutchinsonian Ratios"— an observed minimum scaling threshold of niche differentiation seen in several natural predator populations. Similar minimum values were not seen between the sexes of either species suggesting an absence of sexual niche dimorphism. Geometric morphometric analysis of the mandibles revealed two distinct regions subject to selective adaptation: the distal region of the mandible (including the apical incisor) was consistently sexually dimorphic between the examined species while the proximal region involving the terebral teeth showed interspecific differences independent of sex and likely associated with prey processing, further supporting the hypothesis of niche partitioning between the two species but not necessarily between the sexes.

The magnitude of sexual size dimorphism was found to be constant within Cicindelinae regardless of species body size. Behavioral analysis of mating established 
that $O$. audouini and $O$. dejeanii have average $( \pm \mathrm{SD})$ mating durations of $10.6( \pm 1.8)$ and $29.4( \pm 5.6)$ hours, respectively. Time of initiation of mating (whether morning or evening), food deprivation and operational sex ratio did not have any statistically significant effect on mating duration for either species.

The absence of effect operational sex ratios on mating duration by suggests that mate guarding may not be a universal factor for all tiger beetles and, instead, syncopulatory courtship, as opposed to pre- or post-copulatory courtship, as a female-choice reproductive mechanism may serve as a better explanation for the mating behaviors seen in Omus. 


\section{Dedication}

This dissertation is dedicated to:

...The countless animals sacrificed intentionally or incidentally during my research. You will be neither taken for granted nor forgotten.

...S. Alys (Jones) LaRoe. Your experiences will be neither taken for granted nor forgotten. 


\section{$\underline{\text { Acknowledgments }}$}

Professionally, I must thank Portland Parks and Recreation for permitting me to do research at Powell Butte Nature Park, Oregon with special thanks to M. Hughes, PPR east-side ecologist. Additionally, I thank Dr. C. B. Knisley for his help with tiger beetle biology and valuable suggestions as well as Drs. M. L. Zelditch and D. L. Swiderski for their patience and willingness to explain the fundamentals of geometric morphometrics. Also, in recognition that field-work is never as simple as expected, no matter how well planned, infinite appreciation must be given to J. Jorgenson and M. Mattsson for their invaluable assistance in the forests of Powell Butte. Special gratitude is extended towards R. Treece and her co-workers at the Office of Graduate Studies here at Portland State University for helping me navigate the bureaucracy and follow university standards. I recognize and am grateful for the alumni contributors and faculty distributors of the annual Forbes-Lea Endowment for providing partial funding of this project. I am also indebted to my committee and all of the other members of the faculty who have put up with my questions and granted me advice-I am your intellectual progeny.

Personally, endless gratitude and appreciation must be bestowed on J. Boone, L. Henderson, E. Michel-Marks and L. Tuor and everyone else currently working in or who has passed through the Biology Prep Room and Biology Office at PSU. Both O. Kulander and E. Hart deserve special mention for being my sisters in science in the Ruedas lab as well as my dear friends. Additionally The Bug Chicks, J. Honaker and K. Reddick, have earned a special place in my heart for reminding me that arthropods can still be neat. 
Transcending the professional and personal, I must thank all of my teachers (even if you did not know you were my teacher) and am even more indebted to all who have been my students. A special recognition must be made to the plant genus Camellia. I couldn't have done this without you.

Finally, an enormous expression of acceptance and adoration to all my friends and loved ones, essentially anyone I've (1) hugged, (2) complained to or (3) gotten drunk with since starting PSU. 
$\underline{\text { Table of Contents }}$

Abstract $\quad$ i

Dedication iv

Acknowledgments $\quad \mathrm{V}$

List of Tables viii

List of Figures $\quad \mathrm{X}$

Chapter 1: Introduction 1

$\begin{array}{ll}\text { Chapter 2: Ecomorphology } & 47\end{array}$

Chapter 3: Ethogram and descriptions of behaviors 83

Chapter 4: Mating, Sexual size dimorphism and mating duration 109

$\begin{array}{ll}\text { Chapter 5: Conclusions } & 134\end{array}$

$\begin{array}{ll}\text { Tables } & 143\end{array}$

$\begin{array}{ll}\text { Figures } & 179\end{array}$

$\begin{array}{ll}\text { Literature cited } & 200\end{array}$

Appendix A: Gross morphometric data as CSV file 215

Appendix B: Data and computer files for geometric morphometric analysis of mandibles including tps-format of raw coordinate data and NTS-format of sliders or semi-landmark 220

Appendix C: Data used for mating duration analyses in CSV file format 263

Appendix D: Laboratory husbandry of wild-caught Omus audouini and $O$. dejeanii 266

Appendix E: Microdissection of Omus aedagus 268 


\section{List of Tables}

Table 1.1: Global species biodiversity estimates and percentage of that diversity due to insects.

Table 1.2: Stages in which reproductive success can be manipulated and examples of mechanisms.

Table 1.3: Carabid beetles known to engage in dorso-ventral mating.

Table 2.1: Descriptions of morphometric features and abbreviations use in analysis.

Table 2.2: Summary statistics for individual characters.

Table 2.3: Body length and mandible ratios for species and sexes.

Table 2.4: PC scores (eigenvectors) and eigenvalues of PCA of overall body morphology for both species of Omus.

Table 2.5: PC scores (eigenvectors) and eigenvalues of PCA of overall body morphology for individual species of Omus.

Table 2.6: Reduced major axis linear regression results for allometry of antenna length and mandible length relative to body length.

Table 2.7: Landmarks defined for geometric morphometric analysis of mandibles.

Table 2.8: Singular values (SV) and percent explained for relative warps (RW).

Table 2.9: Results of regression analyses for scaling.

Table 2.10: Results of sexual size dimorphism (SSD) for O. audouini and O. dejeanii.

Table 2.11: Hotelling-Lawley/Hotelling's $\mathrm{T}^{2}$ test for sexual shape dimorphism.

Table 2.12: Summary of proximal and distal terebral teeth angle measurements and curve of the incisor.

Table 2.13: Results of Tukey HSD multiple comparisons of means ( $\alpha=$ 0.05 ) for terebral tooth angles and outer curvature of incisor. 
Table 3.2: Duration of male thrusting episodes during amplexus.

Table 3.3: Features speculated to confer defensive advantage for Omus.

Table 4.1: Body length results of two species of Omus and tiger beetle species used for evaluating trends of sexual size dimorphism.

Table 4.2: Results and summary statistics for $O$. audouini and $O$. dejeanii.

Table 4.3: Mean mating duration for $O$. audouini and $O$. dejeanii for time of initiation experiments (in minutes and hours).

Table 4.4: Regression model details for mating duration for O. audouini.

Table 4.5: Analysis of variance table for best-fit multiple regression model of mating duration in hours for $O$. audouini.

Table 4.6: Pearson correlation coefficients between individual predictor variables and mating duration in hours for O. audouini and O. dejeanii.

Table 4.7: Attempt to determine whether mating duration can be presented as a function of mass as inferred by the $3 / 4$ scaling law.

Table 4.8: Details of pairings for 2012 food deprivation experiments.

Table 4.9: Best-fit multiple regression model details for mating duration for food deprivation experiment.

Table 4.10: Details of pairings for 2012 operational sex ratio experiments. 175

Table 4.11: Results of effects of operational sex ratio on mating duration. 176

Table 4.12: Individuals collected over three years: catch totals and sex ratios.

Table 4.13: $\chi^{2}$-test for equal proportions of sexes over three years. 


\section{List of Figures}

Figure 1.1: Two shapes where the greatest width is the same but points used to define greatest width are not homologous.

Figure 1.2: Rensche's rule of among species trends in sexual size dimorphism, paraphrased as male body size shows greater variation than female body size.

Figure 1.3: Hypothetical allometric scaling of sexual size dimorphism.

Figure 1.4: Cicindeline phylogeny (from Maddison et al. 1999 \& Vogler et al. 2005)

Figure 1.5 Powell Butte Nature Park, Portland, Oregon. 45²9'14.5'N

$122^{\circ} 30^{\prime} 06.5^{\prime \prime} \mathrm{W}$. Contours at ca. 15 meters, summit (marked as $\otimes$ ) ca. 190 m.

Figure 1.6 The author at Powell Butte, checking pitfall traps, 27 June 2012.

Figure 2.1: Morphometric features measured for gross anatomical analysis.

Figure 2.2: Univariate results of overall morphometric analysis of gross anatomy.

Figure 2.3: Results of the Principal Component Analysis (PCA) of gross anatomy for two species of Omus.

Figure 2.4: Results of the Principal Component Analysis (PCA) of gross anatomy for two species of Omus.

Figure 2.5: Linear measurements taken of left mandible and pronotum and elytra.

Figure 2.6: Left mandible of Omus dejeanii showing general mandible structures.

Figure 2.7: Geometric morphometric landmarks used in the mandibular analysis.

Figure 2.8: Percent variation among individuals explained by number of relative warps. 
Figure 2.9: Relative warps analysis showing RW1 and RW2. 193

Figure 2.10: Thin-plate spline visualization of travel along RW1 ("sex"). 194

Figure 2.11: Thin-plate spline visualization of travel along RW2

("species"). 195

Figure 3.1: Screen-captures of post-copulatory intersexual cannibalism in Omus dejeanii and photograph of male remains.

Figure 4.1: Trend of sexual size dimorphism across eight species of tiger beetles, reduced major axis regression of $\ln$ (male body length) onto $\ln$ (female body length).

Figure 4.2: Box and whisker plot showing distribution of mating duration observations for O. audouini and O. dejeanii for time of initiation experiment (2011).

Figure 4.3: Mean mating duration (in minutes) for $O$. audouini and $O$. dejeanii under differing operational sex ratios. 


\section{Chapter 1: Introduction}

\section{The importance of food and sex, and what we can learn from insects}

Food, both the acquisition of food and the avoidance to becoming food, and sex are the predominant concerns of most known forms of animal life. Food is necessary for maintaining the individual organism and successful reproduction, the maintainance of the genetic lineage, is a consequence of selection of features that contribute to an individual's fitness. These necessities result in intense evolutionary selective pressures that shape all levels of the organism from sub-cellular to morphological to behavioral.

Recent estimates of species diversity suggest that $70-76 \%$ of all described animal species are some kind of insect (Bailie et al. 2004, Chapman 2009, Vie et al. 2009; Table 1.1). Within that diversity is seen nearly every form of trophic interaction, diet (insects, as a group, are capable of nutritionally exploiting nearly every known form of organic substance, Triplehorn \& Johnson 2005), and reproductive strategy known for animals, in addition to many novel forms known only for insects (see Thornhill \& Alcock 1983 for the seminal review on insect reproduction). As the dominant form of animal life, in terms of both taxonomic and ecological diversity, insects are invaluable for understanding ecological and evolutionary trends across nearly all fields of biological study.

\section{Review of the concepts of niches, resources and resolving competition}

\section{A. Niche, resources and competition: a general review of relevant topics}

The discussion of the needs and available resources for an animal necessarily brings up the concept of the niche. Unfortunately, "niche" in itself is not without variable 
interpretations (see Vandermeer 1972 for a review and history of the concepts). Early usage largely applied to the Grinnellian niche, using the term to broadly refer to a range of habitat conditions including geographic, biotic, and abiotic factors used to explain the limits imposed on an organism's distribution (Grinnell 1917).

Current, casual use of the term ecological niche in contrast typically references the Eltonian niche concept, where a niche is defined by the organism's relationship to other members of the local community, “...its relations to food and enemies" (Elton 1927), i.e., the role, or "job" of the organism within the community, e.g., is the organism a primary predator, or a decomposer? The Eltonian niche concept focusses almost entirely on biotic factors affecting the organism, specifically its role in “...the transfer of organic matter..." through the ecosystem (Elton \& Miller 1954)

Hutchinson (1957) proposed an alternative to the popular Eltonian niche concept. Hutchinson envisioned the environment as an $n$-dimensional Cartesian coordinate system with each axis representing an independent environmental factor relevant to the survival of the organism in question, e.g., humidity, temperature range, caloric (or prey) availability. The niche of the organism is defined by the specific (hyper)volume within that $n$-dimensional space that delimits the range of environmental conditions, both biotic and abiotic, that would allow a population to survive indefinitely (Hutchinson 1957).

Unsurprisingly given the diversity of life, the needs of individuals of different species may overlap within a given singular geographic areas, resulting in a competition for resources. As such, resource competition between or among species (interspecific), as opposed to competition within a population of a single species (intraspecific), may exist as a trophically horizontal (i.e. intraguild) or even trophically independent 
relationship (Hutchinson 1959, Schoener 1974, Polis et al. 1989).

At times, the resource in question may be the life of an organism. For animals, food acquisition is very often a predator-prey (or parasite-host) relationship — excepting detritivores - in contrast to the other kingdoms of life, which may involve primary production and depend on abiotic sources of energy. The evolution of a diet requires adaptations relating to locating, ingesting, and digesting the food. This involves adaptation of the neurological and sensory systems so the animal is sensitive to cues relating to the food source, anatomical modifications allowing the food to be handled and ingested (ranging from simply placing the mouthparts against a liquid food source and creating a slight suction, to the weapons and behaviors necessary to hunt and subdue prey animals in violent combat), as well as physiological adaptations to maximize nutrient extraction within evolutionary constraints, e.g., animals remain incapable of digesting cellulose without microbial symbionts.

\section{B. Ecological and (co-)evolutionary resolutions to maintaining populations}

While the competitive exclusion principle states that complete competitors cannot co-exist (Gause 1934, Hardin 1960), nature rarely functions within the absolutes of mathematical models, the models themselves being dependent on a number of assumptions that may or may not be valid. Similarly, predators are rarely $100 \%$ efficient or solely dependent on a single prey species. Consequently, as already suggested, populations may persist in a location despite competition or predation due to a combination of non-evolutionary mechanisms relying on the population ecology of the organisms as well as, over generations, adaptive evolutionary mechanisms. 
Given these challenges, species must respond evolutionarily or face extinction. Population displacement but not necessarily co-evolution between competitors is generally the expected outcome except in communities with low species-diversity (Connell 1980). In contrast, predator-prey relationships have the strong potential to elicit a co-evolutionary response due to the obligatory interdependency of the interaction (predators are obligated to pursue the prey while prey are obligated to respond to the predation, each generating strong selective pressure on the other; Connell 1980). Few organisms quietly acquiesce to being eaten (excepting certain plants, fungi, and parasites that capitalize on ingestion for propagule dispersal) and most resist predation through a variety of means including chemical, behavioral and anatomical defenses. This may result in a trophically vertical "evolutionary arms race" of predator and prey "countering" one another as a novel adaptation conferring a temporary advantage becomes established throughout a population (the "Red Queen" hypothesis of Van Valen 1973).

An observed trend ascribed as a potential (and partial) mechanism for niche partitioning is character displacement: populations of ecologically similar sympatric species often show accentuated differences relative to allopatric populations, the competition among ecologically similar organisms generating selective pressure to differentiate (Brown \& Wilson 1956, Dayan \& Simberloff 2005).

This phenomenon of character displacement has been identified across numerous systems (reviewed in Schoener 1974, and Dayan \& Simberloff 2005), such as body size in snails correlating with food particle size (Fenchel 1975) and mandible gape size in tiger beetles relating to prey size (Pearson \& Juliano 1991) but, perhaps, most famously illustrated in the diversity of the many species of Geospiza, Darwin's finches, in the 
Galapagos Islands (Lack 1947, Grant 1986). While the finches exemplify resource partitioning through morphological variation (beak size and shape associated with diet), resources within the overarching environment may be divided through differences in nonanatomical characters such as activity cycle (temporal partitioning), or spatial segregation (microhabitat partitioning; Schoener 1974, Dayan \& Simberloff 2005).

By itself, absolute body size is a valuable character related to resource usage and relative body sizes of sympatric, potentially competing species is often used to indicate niche partitioning. Hutchinson (1959) proposed that differences in body size along a "critical" threshold factor of ca. x1.3 (e.g. $10 \mathrm{~mm}, 13 \mathrm{~mm}, 17 \mathrm{~mm}$, etc...) allowed resource partitioning and coexistance among otherwise potential competitors. These "Hutchinsonian ratios" are often used as evidence of adaptation and specialization in response to past competition pressure and popularized to the point of being regarded as a general ecological rule but, upon examination, are not without exceptions (Simberloff \& Boecklen 1981). Niche partitioning solely through body size differentiation along Hutchinsonian ratios assumes that resource use is correlated with body size, this may be both predicted and has been observed to be a phenomenon restricted to predators and predominately under the circumstances of broad prey size-class distributions (Wilson 1975).

Character divergence among competing predators is often assumed based on partitioning among available prey size classes with each predator specializing on its optimal prey size. Early attempts at addressing character divergence due solely to preysize class predicted the phenomenon to be rare in nature and only a few confirmed examples were known (Wilson 1975). The reasoning for this prediction was attributed to 
the rapidly increasing disadvantages of a predator attempting to take a prey-item above that predator's optimal size-class in conjunction with the relatively few disadvantages existing for a predator taking prey of a sub-optimal size class, i.e., larger animals have an easier time consuming prey classes unavailable to smaller predators in addition to being able to access the prey size classes used by the smaller predators (Wilson 1975). The preceeding statement may be regarded, in part, to conflict with Optimal Foraging Theory (OFT). OFT supposes that, in the presence of a range of food options, an animal will pursue the foraging options that maximizes net benefit to the individual (MacArthur \& Pianka 1966). Maximizing individual benefit involves avoiding options with undue costs such as excessive handling time (manipulating sub-optimally sized prey or processing to bypass inedible tissues) or undue personal risk (venoms and claws contribute towards making an animal a sub-optimal foraging decision) with however there is the caveat that diet specificity may become relaxed as the density of optimal prey decreases (Pyke 1984), thus allowing for situations where large-bodied predator may pursue a range of sub-optimally sized prey while smaller predators remain unable to tackle prey-classes above a size threshold. Importantly, Wilson (1975) assumes a situation where body size alone determines prey-handling ability. Body-size, by itself, may not entirely delimit prey-handling ability. This has been described in communities of sympatric tiger beetles: the mandible length rather than solely body size defines mouth gape that, in turn, determines prey base (Pearson \& Mury 1979, Pearson \& Juliano 1991). Rather than Huthinsonian ratios occurring among body sizes, scaling ratios of x1.2-1.3 were found among the mandible lengths (Pearson \& Mury 1979, Pearson \& Juliano 1991). 


\section{Sex as a resource}

In contrast to many other resources, the opportunity to successfully reproduce is largely an intraspecific competition — hybrid matings between different species are assumed here to rarely outperform same species matings. Additionally, reproduction carries selective pressures that may be at odds with the survival of the individual but contributes to the fitness of the individual by increasing the probability of viable offspring, e.g., sexual selection (Darwin 1859, Gould 1974). The conflict between the survival of the individual and the persistence of the genome of the individual through generations is further exacerbated by the intrinsic difference in parental investment between the sexes: all other things being equal, eggs are more costly than sperm (Parker et al. 1972, Trivers 1972). Unequal investment results in different selective pressures being exerted on the sexes, generally modeled such that the sex with the lower parental investment attempts to maximize the number of partners while the sex with the higher parental investment attempts to maximize the quality of partners (Bateman 1948). We will return to and expand upon this topic during the review of insect reproductive behaviors (Chapter 1, section D.).

\section{A review of general concepts in ecomorphology and morphometrics A. Ecomorphology}

Shape and size define the morphology of an organism and, consequently, define the potential niche-space available to the organism (i.e. "fundamental niche" sensu Hutchinson 1957; Futuyma \& Moreno 1988, Wainwright \& Reilly 1994). Regarding the body as a set of tools allowing the organism to interact with its environment, differences 
in morphology translate into different "tools" that, in turn, imply different resource use. However, morphological differences between sexes or among species demand investments of time and energy to allow differential developmental processes as well as the genes to coordinate these developmental events. Consequently, shape and size are under genetic and developmental constraints in addition to the constraints imposed by the necessary function of a given morphology.

Shape, in regards to both the shape of specific organs as well as the general form of the body, is a character that may be measured as distinct and independent from size. While shape may be constrained by limits imposed by size, shape and size constitute two separate features that may be analyzed separately. Changes in shape depend on localized, differential developmental patterns, i.e., certain areas either grow faster or for a longer time than other regions. The functionality of a given organ, in terms of its applied use and efficiency towards that application, is largely but not entirely delimited by its shape: an appendage used for even occasional swimming requires a flattened profile, a cylindrical cross-section simply will not do for a paddle. Likewise, an organ used for piercing materials simply cannot function without a pointed end and enough structural rigidity to pass through the material to be penetrated.

Size, the other factor defining morphology, also imposes functional limitations, particularly through the relationships among linear dimension, volume, and surface area where each increases at distinct rates. Consequently a structure may be prohibitively massive and unwieldy or unacceptably fragile (or too small or gracile) to perform above or below a certain mass threshold. Additionally, constraints may impose a specific size range, genitals in animals with internal insemination being a quintessential example for 
very narrow size tolerances. Furthermore, the non-linear relationship of surface area to volume may also impose limitations in the case of tissues dependent on effective diffusion (e.g. highly metabolically active tissues requiring a high surface area-to-volume ratio or organs used for filtration or gas exchange) or organs sensitive to desiccation or heat loss (e.g. exposed mucous membranes of desert animals requiring a low surface area-to-volume ratio).

While morphology largely delimits current ecology, morphology is not necessarily an adaptive result of an organism's ecology. Genetic, developmental and physical constraints (often, but not necessarily appropriately referred to as phylogenetic constraints, see Losos \& Miles 1994) impose themselves on an organism (Gould \& Lewontin 1979). Consequently, synapomorphic traits stand the possibility of being independent of local selective pressures while homoplasic traits suggest true adaptations in response to their habitat. As the "tool" defines the range of allowable resources, the tool-resource relationship may be the result of a pre-existing and relatively invariant morphology rather than the morphology being an adaptation to an available resource, e.g., did a beetle evolve a flattened body shape to exploit unexploited crevices or did the already flat beetle opportunistically capitalize on narrow crevices as a habitat across multiple geographic areas? In such cases, a robustly supported phylogeny showing ancestral and derived states within the lineage is invaluable towards answering the question of whether traits represent exaptations or are true adaptations.

Sexual dimorphism has been a persistent point of interest among biologists and may be considered separate from other ecomorphological topics. Sexual dimorphism arises out of evolutionary pressures unique to the particular sex rather than environmental 
pressures driving ecomorphological adaptations for the species. Differences in size and shape between the sexes of a species are widespread throughout both plant and animal kingdoms (e.g. Ruedas et al 1994, Blanckenhorn 2005, Blanckenhorn et al. 2007a \& b, Fairbairn 1997, Lloyd \& Webb 1977, Shine 1989). The necessity of differences between features directly involved in reproduction (i.e. sexual organs such as genitals and gonads) is largely self-evident and invites relatively little inquiry. More compelling questions are inspired by those features not directly involved in copulation but that regardless, still exhibit sexual dimorphism.

\section{B. Morphometrics and the recent "revolution"}

Morphometrics is the analysis of size and shape. When applied to biological systems, morphology contributes to our understanding of the development, physiology, ecology, and evolution of organisms. The application of robust mathematical principles to questions of biological forms and the physical consequences of shape was largely set forth in D'arcy Wentworth Thompson's 1917 On Growth and Form, explicitly stating that “...no organic forms exist save such as are in conformity with physical and mathematical laws" (Thompson 1917). Since 1917, advances in processing ability has allowed a wide variety of multivariate analytical methods to be developed allowing a researcher to address increasingly complex questions.

Traditional morphometric analysis generally relies on basic Euclidian geometric principles using linear distances and angles to describe morphology, including both size and shape (Bookstein 1978). Typically, distances between landmarks are measured from specimens or images of specimens. Non-landmark methods based on matching a 
mathematical function (e.g. elliptical Fourier analysis) exist for forms lacking definable, discrete landmarks such as curves or outlines (Rohlf \& Archie 1984, Rohlf 1990).

The development of increased processing ability and statistical techniques enabled analysis of large, multivariate datasets, allowing the researcher to not only identify trends but also estimate the importance of any given measurement (particularly for landmark-based analyses) in relation to the overall analysis (e.g. eigenvectors of principal component analysis) and minimize redundant or non-influential variables for the analysis (Rohlf 1990).

Landmark-based geometric morphometrics involves methods to statistically describe and compare the shape of a structure by resolving it into a series of discrete landmarks described by Cartesian coordinates (Adams et al. 2004, Slice 2007, Zelditch et al. 2004). Geometric transformations are then used to align, scale and superimpose the configurations to remove the non-shape factors of size, location and rotation in a process known as General Procrustes Superimposition (GPS, or General Procrustes Alignment, GPA) (Rohlf 1999). Once scaled and superimposed, multivariate statistical techniques can be used to test for differences among individuals and classes and regression of shape variables on other variables. Additionally, the differences in shape may be graphically visualized with a thin-plate spline, a mathematical function used to represent shape change as a deformed grid (Bookstein 1989). While a full explanation of the theory and application of geometric morphometrics exceeds the bounds of this dissertation, a more detailed exposition on the specific methods of landmark-based geometric morphometrics relevant to this study (including sliding landmarks) is presented in chapter three to preface the morphometric analysis of the Omus mandibles. 
Arthropods are particularly well suited to morphometric analysis because their rigid exoskeleton allows a researcher to avoid the trouble of soft tissue distortion inherent with other animals (Daly 1985). Insects undergoing complete metamorphosis (holometaboly) are even more conducive to study because there is the single transition into maturity so that an adult form is fixed and will not grow or change short of traumatic injury (Triplehorn \& Johnson 2007). However no method is perfect and the joints of arthropods are composed of unsclerotized cuticle and flexible to allow movement so an error term, however slight, is unavoidably introduced for every compressible element (joint) present between landmarks.

\section{Review of insect mating behaviors}

\section{A. General reproductive anatomy for sexual animals}

Sexual reproduction involves the fusion of gametes that then develop into discrete individuals. As such, essential reproductive anatomy involves organs that (1) produce gametes and then (2) allow passage of gametes to allow for fusion between (or among) gametes. Gamete passage may be either unidirectional, as in the case of external fertilization where eggs and sperm are cast into the environment, or bidirectional, as in the case of internal fertilization where sperm is allowed to enter the female tract and then fertilized eggs (or offspring in some stage of development) to leave the tract. The basic organs of reproduction, gamete-producing organs and gamete-transmitting ducts, are homologous and arise from the same embryonic germ cells that differentiate during the course of development (Kaulenas 1992). 


\section{Evolutionary pressures shaping insect mating behaviors}

At the heart of animal sexual reproductive strategies is not only the competition for mates (intrasexual competition) but the competition between mates (intersexual competition) arising from the conflicting agendas between females and males. With the development of anisogamy, where the sexes produce differently sized gametes, there is a difference in the degree of parental investment for successful reproduction (Eberhard 1996, Birkhead 2000, Simmons 2001). Darwin (1859) explicitly differentiated between natural selection and sexual selection, indicating that natural selection acts to increase the viability of the individual while sexual selection increases the reproductive success of the individual (Simmons 2001). Due to the different degrees of investment between the sexes - males producing metabolically inexpensive sperm and females producing costly eggs to use the simplest case - there are different selective pressures regarding what counts as beneficial for each sex. All other things being equal, males benefit most by maximizing the number of mates (their time spent mating being the valuable investment) while females benefit through maximizing the quality of mate and, supposedly, the subsequent quality of the offspring (the production and development of their gametes being the valuable investment) (Birkhead 2000). Consequently, males most often tend to compete with other males for access to females (classical sexual selection sensu Darwin 1859) while, in turn, females impose a suite of demands and minimum criteria prior to allowing successful mating with a given male (female choice). Sexual selection as a general-case intrasexual competition for mates is also known to exist as female-female competition in systems where the males heavily invest in the reproductive effort such as the male pregnancy of Pipefish (Syngnathidae) (Berglund et al. 1986) or the sex role 
reversal seen in the Eurasian Dotterel, Charadrius morinellus that includes female lekking and exclusively male parental care (Owens et al. 1994).

The operational sex ratio (OSR) of the population, the proportion of sexually receptive males to females (Emlen \& Oring 1977), becomes increasingly male-biased as females are mated and, temporarily at least, become non-receptive (Johnstone et al. 1996). This drive towards a male-biased OSR predicts that the unmated females, can afford to be extremely choosy while males grow more opportunistic. However, the time investment to find alternative mates is more costly for males than for females and male choice may be expected to be a factor not only of parental investment but also mate "processing time", the amount of time is takes to encounter and evaluate a potential mate (Johnstone et al. 1996).

The role of the male in classical sexual selection has been widely assumed and investigated in a general sense but recent years have appended sexual selection theory to reframe it from a competition between males for access to the female to one where the males are competing, specifically, for access to the female's gametes (Eberhard 1994 \& 1996, Birkhead 2000). This shift in focus from the whole organism to the fate of the gametes predicts the existence of both overt and cryptic mechanisms whereby the competition between males and between the sexes continues beyond the act of copulation and includes other mechanisms such as indirect mate guarding (plugs), sperm competition by precedence, and female control of sperm usage (Eberhard 1996, Birkhead 2000, Simmons 2001; Table 1.2).

\section{Male-male competition, sperm competition and mate guarding}


The existence of sexually dimorphic "bizarre" structures have historically both puzzled and inspired researchers (Gould 1974). The tails of peafowl (Genus Pavo), the horns of stag beetles (Family Lucanidae) and the exceptionally disproportionate antlers of the extinct Irish Elk (Megaloceros giganteus) were obviously handicaps to the bearers but the assumed economy of nature insisted that the benefit must, somehow, outweigh the cost of development, maintenance and general burden of these features. The advent of a cogent theory of sexual selection gave a mechanism to these features that reconciled their cost to the individual by contributing to the bearer's reproductive success. In addition to allowing for direct competition with other males (e.g. horns or claws for combat), there is often a genetic correlation between the presence and development of these traits in males, and preference for the traits by females (Simmons 2001). Thus, the selection pressure is ultimately for the appearance of quality in hopes that the signal remains true and correlates with the actual quality of the mate (an honest signal). Ultimately, the consequence is that traits may experience exaggerated development until the viability cost finally outweighs reproductive benefit, imposing a limit on the magnitude of that trait (Fisher 1915, Simmons 2001).

In addition to combat, males may compete against other males through less direct means and still gain reproductive advantages. Phenotypic polymorphisms, whether morphological and behavioral, are various forms within the population, each capitalizing on its own behavioral competitive strategy ranging from direct, overt competition and territory defense to more subtle means such as "sneaker" males (Gross 1996; In the marine isopod, Paracerceis sculpta: Shuster 1987, Shuster \& Wade 1991; In the sandpiper, Philomachus pugnax: Lank et al. 1995). 
However, less elaborate interactions are assumed to be more common among animals. Post-copulatory mate guarding (as opposed to pre-copulatory mate guarding where the male guards an immature female, Thornhill \& Alcock 1983) is a common form of male-male competition where the male remains near or in contact with the female post-insemination to physically prevent subsequent mating and insure his own paternity (Parker 1974, Alcock 1994). This not only implies promiscuity as an innate component of the mating system - why guard the female if she will not mate again? - but also infers the existence of sperm competition and, specifically, last-male advantage where the last male to mate fertilizes the majority (if not the entirety) of the brood (Parker 1974, Alcock 1994).

\section{Mate choice and courtship}

The idea that males would compete with other males for access to mating opportunities was established along with the idea of natural selection. It was not until quite a bit later that the idea of female choice was considered and explored - the possibility that the females themselves could generate selective pressure by showing preference for one mate over another and, at its strongest, may even abort an otherwise successful reproductive event in the case of an undesirable mate. Female choice in mating occurs whenever a female exhibits a different response to mating efforts by conspecific males resulting in a change in the reproductive success, or fitness, of that male (Eberhard 1996). Under Bateman's principle, females generally benefit most strongly through qualitative selection of mates as contrasted by male strategies, which often emphasize the overall quantity of mates (Bateman 1948, Thornhill \& Alcock 1983). 
Consequently, it may be assumed that females of a sexually reproducing species possess mechanisms of at least moderate efficacy that allow for differential access to her gametes by potential mates.

Under conditions of shared (care for the offspring is split equally between the sexes) or reversed parental care (males predominately care for the offspring), the direction of choice is also split or reversed. Females may display ornamentation to attract males and, in shared care reproductive systems, mutual choice mechanisms may develop where both sexes display and exhibit choice. The argument that female ornamentation is an artifact of genetic correlation and the females are displaying a trait that is primarily under female-choice selective pressures may be countered with the observation of female crypsis - the de-emphasizing in females of traits shared by both sexes. The mostly shared genome of the sexes means a degree of genetic correlation is unavoidable however hormone-mediated developmental processes are responsible for the degree of expression for any given trait. As such, the genes for the ornament may be shared however the expression of that trait is notoriously plastic and strongly subject to the needs of the sex (Amundsen 2000).

Regardless, both sexes of animals have been documented to have the ability to influence their reproductive success throughout the mating effort, starting with mate location all the way to raising the offspring (Table 1.2). Those behaviors that influence reproductive success beyond the act of mating may be termed "cryptic"-cryptic in the sense that the actual results of the mating, the success of the offspring, are hidden from one partner regardless of whether the short-term act of mating was accomplished (Thornhill 1983, Eberhard 1996). 


\section{Courtship as a choice mechanism}

Courtship signals made by males towards females imply that females have some innate degree of control over the success of the reproductive attempt (Eberhard 1996). These may be signals of (supposed) quality and health (vigorous displays, nuptial gifts), species identity (males are typically less particular on this than females, Thornhill \& Alcock 1983), or may be an exploited "sensory trap" where the male mimics non-sexual stimuli pre-existing in the female that facilitates mating, e.g., male bees and moths producing a "flowery" or prey-plant odor to attract females (Christy 1995, Eberhard 1996). Sensory traps, of course, may be applied to non-reproductive ends as in the case of female fireflies of the genus Photuris mimicking the courtship pattern of light flashes of other firefly genera that are then eaten by the Photuris (Christy 1995).

The timing of the courtship behavior correlates to events during mating where the female has the option to not cooperate with the male's efforts and undermine his reproductive success either through direct refusal to mate or through "cryptic" means where the fate of the offspring outside of the male's control (Thornhill 1983, Eberhard 1994 \& 1996, Birkhead 1998). Hence, there is pre-copulatory courtship where the male vies for initial contact with the female and syn-copulatory courtship where the male attempts to induce the female to allow intromission and insemination. This may be extended to post-copulatory events, particularly where the female are able to lower the proportion of undesired sperm by mating with other males ("swamping"), eject the sperm outright or abort the brood (Thornhill 1983, Eberhard 1996). Among insects, syncopulatory courtship is believed to be common and takes the form of stereotypic 
behaviors not directly associated with the genitals or sperm transmission, e.g., the male tapping and stroking the female with his antennae or legs (Eberhard 1996).

4. Genital morphology as a response to female choice or, why "lock-and-key" is wrong

Male insect genitals are noted for their extreme morphological diversity and specificity to the point of being used as critical characters in taxonomic identity and definition of species (e.g. Horn 1930). The vast diversity of form in the male genitalia and ease of observation due to the external or eversible nature of the organ naturally lends itself to study. The internal nature of female genital and lack of rigid structures makes female genitalic morphology a challenging study and the details of how the female reproductive tract responds to coitus remain poorly understood.

Prior speculation proposed a "genital lock and key" explanation for the observed genitial diversity seen among insects. The belief was that the complex morphology and microanatomical structures are the result of selection for an interspecific reproductive barrier (see Shapiro \& Porter 1989 for a review). However, alternate evidence fails to support this perspective (Eberhard 1985). While interspecific hybridization may indeed be prevented thought mechanical incompatibility preventing intromission or even traumatic injury and fatality upon insertion, e.g., Sota \& Kubota 1998, this appears to be an incidental rather than intended consequence of the mismatched genitalia (Eberhard 1985). While death does indeed impose a strong selective pressure, partner fatality due to mismatched genitalia predominately affects the female. In contrast, the male generally does not invoke any noted selective consequences for his attempt with the exception of a single failed mating attempt out of several. Furthermore, Shapiro \& Porter (1989) note 
that there is little correlation between genital morphology and hybridization success among insects. Rather than mechanical exclusion of heterospecifics, current hypotheses regard genital morphology as the result of cryptic female choice mechanism towards insuring species identity through tactile cues rather than selection for mechanical incompatibility (Eberhard 1985).

\section{Tiger beetles}

\section{A. Tiger beetles: general introduction}

Beetles in general serve as excellent models for research because of their taxonomic, evolutionary, ecological, and physiological diversity. Among the beetles, Order Coleoptera, Carabidae is one of the most diverse of the families and has historically enjoyed a large degree of popularity among researchers because of their ease of capture, relative ubiquity across habitats, vast diversity of form and ecological significance. Carabid beetles are predominately medium-to-large bodied forest floor predators with a cosmopolitan distribution occupying a gradient of habitat specialization ranging from highly specific to generalist (Brouat et al. 2003, Lovei \& Sunderland 1996, Thiele 1977). While many species are capable of flight as sexually mature adults, they are generally reticent to take wing (Triplehorn \& Johnson 2005) or, occasionally, altogether incapable of flight as in the case of apterous (wingless) or brachypterous (reduced wings) species (often bearing fused elytrae), both forms having evolved multiple times (Lovei \& Sunderland 1996; e.g. beetles of the genera Carabus, Pterostichus, or Scaphinotus). The sub-adult forms of carabids (larva and pupa) are, without exception, wingless and of extremely limited vagility (Triplehorn \& Johnson 
2007). This combination of wide distribution with limited dispersal ability intimately ties beetles to their local environment, making them exceptional models for fine- and largescale examinations of evolutionary adaptation, population structure, ecological disturbance, and habitat fragmentation studies (e.g., Brouat et al. 2004, Drees et al. 2008, Driscoll et al. 2010, Garnier et al. 2004, Keller et al. 2004).

Tiger beetles (Carabidae: Cicindelinae) are a notable subfamily within Carabidae and form a highly speciose group of nearly 30 genera containing over 2,300 species with a nearly worldwide distribution across a wide range of habitats (Cassola \& Pearson 2000, Pearson \& Vogler 2001). The majority of the diversity of the subfamily resides in the genus Cicindela sensu lato, a complex of over 800 recognized species that are generally endemic to relatively open areas such as scrub forests, grasslands, sandy areas, and salt flats. However several of the minor genera retain the ancestral forest floor habitat for Carabidae (Pearson \& Vogler 2001). Evidence of the monophyly of Cicindelinae and their placement within the family Carabidae has been well supported through repeated studies using both nuclear and mitochondrial loci (Maddison et al. 1999, Maddison et al. 2009, Shull et al. 2001, Vogler \& Pearson 1996). In addition to being morphologically distinctive and often visually striking, tiger beetles have been proven useful as models in research on behavior, conservation, ecology, evolution, and physiology (e.g. Dreisig 1980, Freitag 1974, Fielding \& Knisley 1995, Knisley \& Haines 2010, Knisley \& Juliano 1988, May et al. 1986, Pearson \& Cassola 2005, Pearson \& Cassola 2007, Schultz et al. 1992).

\section{B. Tiger beetle taxonomy}


The organization of genera and species within Cicindelinae is broadly supported through published studies, however the bulk of interest in tiger beetle systematics stems from a narrow community and, hence, attention is given to the more prominent taxa and general structure of the subfamily leaving the placement of minor taxa poorly resolved. To date, little molecular work has been done to validate proposed species identities among the basal lineages or to resolve relations among and within those groups. The currently accepted structure of Cicindelinae places Cicindela sensu stricto, the most speciose genera, as the most derived with several genera associated with Cicindela sensu lato (e.g. Odontocheila, Oxycheila) as sister taxa. A number of genera have as yet unresolved polytomic relationships (Vogler \& Pearson 1996, Pearson \& Vogler 2001, Barraclough \& Vogler 2002). Basal to Cicindela sensu lato fall a handful of predominately North and South American genera with relatively low extant diversity: Megacephala (ca. 60 pantropical species but includes the North American Tetracha), Amblycheila ( $<10$ species, restricted to Western North America and Northern Mexico), Pinochile (monotypic, found in Chile and Argentina), and Omus ( $<15$ species, exclusive to the west coast of North America) (Pearson \& Vogler 2001).

\section{Life cycle and ecology of tiger beetles}

As with all coleoptera, tiger beetles are holometabolous, meaning that they undergo a single, complete metamorphosis as they transition from an immature larva to a sexually mature adult via a quiescent pupal stage, each life stage having unique needs in terms of diet or habitat requirements. This is in contrast to other developmental strategies that carry few differences in the requirements between immature and adult forms such as 
hemimetabolous insects that gradually transition from immature larva (or, more appropriately, nymph) to sexually mature adult through a series of instars, or ametabolous insects where the immature forms are not notably different from the mature forms except for body size and genital development. The evolution of discrete life-history stages, each with its own niche, is believed to minimize intraspecific, intergenerational competition, with evidence suggesting the larval form being a modified free-living form of an embryonic developmental stage present in hemimetabolous and ametabolus insects rather than a novel stage (Truman \& Riddiford 1999).

The life cycle begins with a fertilized egg. Eggs are laid singly in individually prepared sites, rather than en masse, and generally on the order of 10-50 eggs per day depending on the species (Knisley \& Schultz 1997, Hoback et al. 2000, Pearson \& Vogler 2001). The oviposition site is critical for egg and larval success. Individual species have their own preference in oviposition site selection, based on salinity, exposure, soil particulate size, and even slope (Leffler 1979, Knisley \& Schultz 1997, Hoback et al. 2000, Cornelisse \& Hafernik 2009, Willis 1967). Oviposition site is of particular importance not only for egg development (moisture apparently being the dominant factor in determining eclosion/hatching, Knisley \& Schultz 1997) but also for the ecology of the larva. Upon eclosion, tiger beetle larva dig burrows into the substrate and act as ambush predators pending metamorphosis, waiting at the mouth of the burrow entrance to capture passing prey items, striking at and consuming nearly any suitably sized arthropod (Willis 1967). Not only are the larvae largely unsclerotized (Knisley \& Schultz 1997) making them prone to desiccation and environmental dangers of injury, predation, infection or parasitism, they are also heavily adapted to a burrowing - rather 
than a cursorial— lifestyle (Pearson \& Vogler 2001) and consequently have little ability to relocate given unsuitable environmental conditions.

Larvae must eat enough to pass through three instars (larval stages) prior to pupating into an adult form, a process that may take from a single season to three years depending on conditions and species (Knisley \& Schultz 1997, Pearson \& Vogler 2001). Pupation generally takes 2-4 weeks after which the adult emerges (Knisley \& Schultz 1997). Adult life spans vary greatly among species and may range from a few weeks after emergence to a year or more, over-wintering between seasons (Knisely \& Schultz 1997, Pearson \& Vogler 2001).

Adults are predominately cursorial and visual hunters and, when able, use flight more as a defensive escape mechanism than for prey pursuit (Person \& Vogler 2001). Most species (the Cicindela species complex) are endemic to exposed habitats such as salt flats, sandy riverbanks, and grasslands, but forest-dwelling and even arboreal species are known (Pearson \& Vogler 2001). Tiger beetles, both larvae and adults, are presumed to be indiscriminately predatory on a wide range of invertebrate prey and known to pursue, subdue, and consume prey based largely on prey size rather than prey taxa (Pearson 1980, Pearson \& Juliano 1991).

Little work has been done identifying predators of larval forms. This is likely due to the difficulty of identifying beetle larva, let alone semi-digested specimens, from stomach contents, as well as the challenges of witnessing the events happening within a burrow. Regardless, ants have been observed digging out and consuming tiger beetle larvae and likely act as prominent predators (Willis 1967). Birds (specifically flickers, Colaptes auratus, see Mury Meyer 1981), several beetles, and particularly parasitoid 
wasps, are also known to be prominent sources of larval mortality (Knisley \& Schultz 1997, Pearson \& Vogler 2001).

The dominant predators of adult tiger beetles have been reasonably well studied, with robber flies and birds targeting tiger beetles in flight, and lizards, amphibians, lycosid spiders (wolf spiders), and insectivorous mammals taking beetles from the ground (Knisley \& Schultz 1997, Pearson \& Vogler 2001). Shrews are specifically known to readily consume Omus and other carabids (Maser \& Hooven 1974), to the point of being a nuisance to pitfall trapping efforts, effectively reducing a week of captures to a few fragments of exoskeleton (van den Berghe 1990, R.K.R. pers. observ.). Moles may also act as predators and adult tiger beetle remains are known from their stomach contents (Scheffer 1911), but the literature has little to say about any possible relationship between moles, or other burrowing mammals, as predators of tiger beetles in either adult or larval forms.

\section{Reproductive behaviors of tiger beetles}

Tiger beetles are opportuistically promiscuous, do not provide parental care past the time of oviposition, and are not known to form pair-bonds after mating (Fielding \& Knisley 1995, Rodriguez 1998, Pearson \& Vogler 2001). Conspicuous pre-copulatory courtship, such as visual displays or ritualized contact prior to mounting, appears to be absent among tiger beetles and copulation efforts are initiated when a male contacts a potential mate and immediately attempts to position himself for coitus (Kraus \& Lederhouse 1983, Shivashankar \& Pearson 1994, Fielding \& Knisley 1995, Rodriguez 1998). A male will attempt to dorsally mount a female and grasp her waist with his 
mandibles, to which the female often responds with a pre-copulatory struggle. This struggle has been interpreted by some authors as a female-choice mechanism whereby the female is testing the quality of the male and only those males who maintain position qualify as suitable mates (Pearson \& Vogler 2001). If the male maintains position, he then everts and inserts his aedagus and the pair engage in coitus with the males generally remaining mounted post-coitus (or between intromission events) as a form of contact mate guarding (Kraus \& Lederhouse 1983, Shivashankar \& Pearson 1994, Fielding \& Knisley 1995, Rodriguez 1998). Behaviors during coitus and non-coital mounting are variable among species and may include the male performing stereotypic thrusting motions with his abdomen or stroking the female with his antennae or his hind legs and are likely forms of coital courtship (Rodriguez 1998). Mate guarding durations are also variable, both among species (Kraus \& Lederhouse 1983, Shivashankar \& Pearson 1994) and even among populations (Rodriguez 1998), and may range from less than an hour (Shivashankar \& Pearson 1994, Rodriguez 1998) to upwards of 16 hours (Pratt 1939) (Table 1.3). Post-mating behaviors, such as serial mating attempts (repeated mating with the same individual) or prior-mate avoidance have not been addressed in the literature. After mating, the female will seek a suitable habitat for ovipositing, typically in a soil type conducive to the construction and maintenance of the larval burrow, individually depositing from only a few to a dozen or more eggs into the substrate (Willis 1967, Pearson \& Vogler 2001).

\section{Night stalking tiger beetles, genus Omus Eschscholtz}

Night stalking tiger beetles (Cicindelinae: Omus Eschscholtz) are endemic 
members of the forest floor community along the North American west coast and are regarded as the least studied genus of tiger beetle (Pearson \& Vogler 2001, Pearson et al. 2006). The basal position of Omus within the greater cicindeline phylogeny is strongly supported through molecular analysis (e.g. Vogler \& Welsh 1997, Maddison et al. 1999, Shull et al. 2001, Galian et al. 2002, Vogler et al. 2005; Figure 1.4), chromosomal traits (Galian et al. 2002), and morphological features such as male genital anatomy and gross morphology (e.g. Horn 1930, Cazier 1942, Leffler 1979). The genus contains at least five recognized species, all of which are exclusive to the forests west of the Cascade Mountain range, and the sole representative of Cicindelinae within the coastal forests of West Coast North America (Freitag 1999, Pearson et al. 2006). Unfortunately, the Omus of Canada are poorly represented in taxonomic treatments, and the forests of British Columbia and Victoria, as well as the Cascade mountain range in general, likely harbor multiple cryptic or altogether undescribed species considering the extremely limited ranges of species such as $O$. cazierii and $O$. submetallicus. At the time of this writing, there are no known reports of Omus endemic to Mexico.

While the typical tiger beetle is imagined as a medium-sized, aggressively flying, and flamboyantly colored beetle, Omus are larger than and morphologically dissimilar from the majority of cicindelines, possessing fused elytra preventing flight and uniformly black cuticles (certain populations express a "bronzy" tone, Leffler et al. 1986). Unfortunately, the biology of Omus remains virtually unknown and limited to speculation and a handful of short, anecdotal reports (e.g. LaBonte \& Johnson 1988, Maser 1977a, Maser 1977b, Maser \& Beer 1971, Pratt 1939, van den Berghe 1990). Omus are believed to have a multi-year life cycle, the larvae spending one or two seasons in the ground 
accumulating enough food to grow and successfully molt into an adult form. Adults may survive only for the single year but may also overwinter and emerge the following spring (Leffler 1979). As the common name of "night stalking tiger beetle" implies, Omus are assumed to be largely crepuscular/nocturnal or at least negatively phototaxic (Pearson \& Vogler 2001, Pearson et al. 2006), however this has been disputed and Omus are known to be active during daytime in both natural and laboratory settings (Maser \& Beer 1971, van den Berghe 1990, R.K.R. pers. observ.).

As with other tiger beetles, Omus are primarily predaceous in both larval and adult form; however their natural prey base is uncertain. There is speculation that native millipedes may form a significant prey base for adult Omus dejeanii (specifically Harpaphae spp. ca. 20-40mm in length, LaBonte \& Johnson 1988). Little work has been done to verify even the most basic details of their life history such as diet and daily activity patterns (e.g. is the majority of the stalking done by night stalking tiger beetles truly done at night?).

\section{Audouin's night stalking tiger beetle, Omus audouini, and the Greater (or Dejean's) night stalking tiger beetle, Omus dejeanii.}

The species Omus audouini and $O$. dejeanii constitute two of three recognized species of Omus occurring in the Pacific Northwest; the third species, O. cazierii, is suspected to be restricted exclusively to the forests of Mt. Ashland and immediate surrounding areas in southern Oregon (van den Berghe 1994).

The species contained within the genus Omus have undergone many taxonomic revisions. Originally described in 1838 by Reiche, $O$. audouini has been variously split 
and consolidated from a variant of $O$. californicus (Horn 1930), into over a dozen species (detailed in Horn 1930, Cazier 1942, and Freitag 1999), before reaching its current status as a single, distinct species (Cazier 1942, Leffler 1979, Freitag 1999). The range and habitat of $O$. audouini includes forests and adjacent meadows west of the Cascade Mountains from northern California, USA, to southern British Columbia, Canada (Pearson et al. 2006). These beetles are considered medium-sized members of cicindelinae (14-18 $\mathrm{mm}$ in length, Pearson et al. 2006), commonly found in suitable habitats throughout the Pacific Northwest, easily captured with unbaited pitfall traps (van den Berghe 1990), and often occuring sympatrically, possibly even syntopically, with the less common O. dejeanii (van den Berghe 1990, Freitag 1999).

Shortly after its initial description in 1838 by Reiche, $O$. dejeanii was briefly split into two separate species, O. robustus Casey (a Washington species occurring north of the Columbia River) and O. foveatus Casey (an Oregon species occurring south of the Columbia River) before being returned to a single species (Horn 1930, Freitag 1999). This beetle is the largest of the Omus species (18-21 mm in length, Pearson et al. 2006) and shares a similar geographic range as O. audouini. However, O. dejeanii is preferentially found in heavy forest with full canopy cover (van den Berghe 1990), although some sources report this beetle as also occurring along coastal beaches among driftwood (Pearson et al. 2000). As with O. audouini, O. dejeanii are readily captured in unbaited pitfall traps placed in suitable habitats (van den Berghe 1990).

The general lack of information on behavioral ecology and life history of Omus makes speculation difficult regarding the potential interactions between $O$. audouini and O. dejeanii. The larger $O$. dejeanii will readily attack and consume $O$. audouini when 
contained together as in a pitfall trap (van den Berghe 1990, R.K.R. pers. observ.), but it is important to note that pitfall traps artificially force contact among species and may not represent natural patterns of interaction. Omus audouini will occasionally be found in the thickly forested habitat of $O$. dejeanii, but the reverse is rarely true. As such, it is difficult to judge the degree of natural contact between the two beetles and whether there exists direct competition for resources (oviposition sites or prey), regular intraguild predation of $O$. dejeanii on $O$. audouini, competitive exclusion of $O$. audouini from forests by $O$. dejeanii or, possibly, little to no regular contact and the presence of one has little influence on the other.

\section{Why Omus and why mandibles?}

\section{A. Context: Relevant articles and prior studies}

The basal position of Omus within the tiger beetle phylogeny (Vogler \& Welsh 1997), their relative ease of capture via pitfall trapping (van den Berghe 1990), the general paucity of knowledge of their natural history and their endemism to the North American west-coast forest floor invertebrate communities, all justify attention and validate their study. However, certain articles, particularly when considered together, highlight recurrent themes in tiger beetle research (both specific to Omus, as well as general to cicindelines as a whole) that identify Omus as a uniquely suitable study subject towards addressing specific knowledge gaps.

- Pratt, R. Y. (1939). The mandibles of Omus dejeani Riche as secondary sexual organs (Coleoptera, Cicindelidae.). Pan-Pacific Entomologist, 15: 95-96. 
While tiger beetles had been known to engage in coitus with the female mounted and seized by the male, Pratt (1939) represents the first explicit description of mating Omus dejeanii and specifically identifies the male mandibles as secondary sexual organs. The article is a short ( $<300$ words), anecdotal description of an unplanned observation by Pratt of an amplexed pair of $O$. dejeanii (=dejeani) in Whidby Island, Washington, during the spring of 1938. He describes the habitat ("under a piece of wood"), notes details of their positions ("Only the posterior pair of legs of the male were resting on the ground") and the time of discovery (02 May 1938 at 5:49pm). Pratt indicated that he placed a covered box over the pair and were found still amplexed as of 10:02 am the following morning (03 May 1938) and noted the supposed duration of that mating episode as being in excess of sixteen hours. He admits to being uncertain whether the male remained in contact with the female throughout the entire duration but noted that the male initially would not release the female despite perturbation (he flipped the pair on their backs and the female righted them) but readily decoupled upon disturbance at 10:02am.

Aside from the pure utility of documenting natural observations, the real significance of this paper rests in 1) the acceptance and inclusion in nearly all subsequent research on tiger beetle mating behaviors, and 2) the complete lack of any follow up research on the mating behaviors of Omus. While Omus are widely recognized as having extended mating times compared to other tiger beetles, there has never been a direct examination of this behavior and, as such, we have no evidence whether the findings of 
Pratt (1939) were typical or anomalous. There is irony in that the final words of Pratt's article are, "Further research along this line would be interesting."

- Freitag, R. (1974). Selection for a non-genitalic mating structure in female tiger beetles of the genus Cicindela (Coleoptera: Cicindelidae). Canadian Entomologist, 106: $561-568$.

Freitag's 1974 article examined multiple tiger beetle species (including geographic variants) and identified a female-specific sulcus (“coupling sulcus”) that corresponded with the placement of the male mandibles during mating for many tiger beetle species. Located on the female mesepisternum (lateral "waist" region of the exoskeleton), he noted consistent, species-specific variation in the depth and shape of the coupling sulci and hypothesized that selective pressures shaped these sulci as a form of rapid species recognition/“lock and key” mechanism in the physiologically stressful environments favored by diurnal tiger beetles, particularly those with several phenotypically similar sympatric species.

Unfortunately, Freitag did not test whether the sulci matched the dentition of conspecific male mandibles or even whether the coupling sulci shape improved the mechanical fit or, conversely, lessened the grip in the case of a poor (i.e. interspecific) match. As such, it may be speculated that the coupling sulci could be either or both of the following: (1) "grip assistance" for conspecific mates, or (2) species recognition cue independent of mechanical fit. Of particular note, Freitag explicitly stated that the basal tiger beetle genera lack the coupling sulci ("Female and male mesepisterna are alike in 
the primitive genera Amblycheila, Megacephala, and Omus", Freitag 1974). He justified this assertion with the assumption that the nocturnal/crepuscular habit of the basal genera render them less agile than more derived genera and as such, were not subjected to the strong selective pressure to generate a mandibular fit mechanism as the structure, “...has not developed in the females since it affords no advantage to them" (Freitag 1974).

- Pearson, D. L. \& Mury, E. J. (1979) Character divergence and convergence among tiger beetles (Coleoptera: Cicindelidae). Ecology, 60: 557-566.

As addressed earlier, character divergence based purely on differences among prey-size classes is predicted to be rare in nature, with only a few confirmed examples primarily restricted to top predators able to access a broad distribution of prey size classes (Wilson 1975; see Section B.4). Pearson \& Mury (1979) found that approximately Hutchinsonian ratios (scaling factors of ca. 1.3X) could be applied to the specific character of mandible length for certain communities of sympatric tiger beetles despite similarities in body sizes.

A series of controlled experiments demonstrated that, while the lower prey size limit was similar among the tiger beetle species examined, median (assumed to represent preferred) prey size correlated strongly and significantly with mandible length $\left(\mathrm{r}_{\mathrm{s}}=0.97\right.$, $\mathrm{p}<0.01$; Pearson \& Mury 1979). They extended their observations to multiple habitats (grassland, permanent pond edge, temporary pond edge, and desert playa/dry lakebed) and found that habitats with narrow distributions of prey sizes but high prey density (permanent pond edges) contained tiger beetle communities with a near continuous, 
narrow gradient of mandible lengths $(<0.5 \mathrm{~mm}$ difference among 11 species, most mandible lengths between $2.0-2.5 \mathrm{~mm}$; a single species had a median mandible length of ca. $1.35 \mathrm{~mm})$. In contrast, habitats with broad prey size class distributions despite low prey density (grasslands) contained tiger beetle communities with species clustered in discrete, non-continuous mandible lengths occurring at a Hutchinsonian ratio of roughly 1.5X (specifically, ca. $1.4 \mathrm{~mm}$, ca. $2.2 \mathrm{~mm}$ and ca. $3.3 \mathrm{~mm}$ ).

This study demonstrated evidence of both prey size-class based character convergence and divergence as dependent on ecological conditions, specifically, prey availability in both size and density. Tiger beetle mandible lengths converged under conditions of narrow prey size diversity and high prey density, the surplus of prey presumably minimizing competitive pressures (hence selective pressures) for food while broad prey size diversity with low prey density generated selective pressures towards character divergence to take place resulting in modest prey size-class specialization and decreased interspecific competition for already limited food. Presumably, the energetic cost of pursuing prey of a sub-optimal size class prevents the largest beetles from outcompeting and displacing smaller beetles (Pearson \& Mury 1979).

Pearson \& Mury (1979) highlighted the importance of a single anatomical organ: the mandibles of the tiger beetle. As the primary tool the beetle has to manipulate its environment, mandibles show evolutionary phenotypic plasticity allowing the tool to be modified depending on habitat conditions relating to available prey and the possibility of competitors. Unfortunately, there was no mention as to whether they tested for the presence of sexual dimorphism in either body length, mandible length, or mandible shape. In addition, they proposed that the cost of pursuing sub-optimal prey sizes 
prevented automatic dominance of the habitat by beetles wielding large mandibles. However, they did not mention the possible effect of habitat complexity on hunting efficiency. While relatively open environments (e.g. pond edges) grant little in the way of cover, grasslands are highly complex, both horizontally and vertically, and smaller body sizes and smaller mandibles may afford an as yet unrecognized advantage, e.g., prey nestled between stalks of grass may be protected from beetles with large mandibles but vulnerable to more modestly proportioned alternative predators.

- Kraus, B. \& Lederhouse, R. C. (1983). Contact guarding during courtship in the tiger beetle Cicindela marutha Dow (Coleoptera: Cicindelidae). American Midland Naturalist, 110: 208-211.

The general behavior of extended amplexus (contact enduring beyond what is required simply for insemination) with the female mounted and grasped by the male was known to be ubiquitous, and, in fact, definitively characteristic, for tiger beetles (Willis 1967, Kraus \& Lederhouse 1983) however the function or otherwise adaptive significance of this behavior had received little to no direct attention. Kraus \& Lederhouse (1983) speculated that the "riding" behavior constituted a form of contact mate guarding, a strategy where the male maintains contact with the female to prevent sperm displacement or dilution by competing males (Parker 1970).

Examining a population of Cicindela marutha, a species of tiger beetle endemic to stream banks, salt flats, and fields of the North American southwest (Pearson et al. 2006), Kraus \& Lederhouse observed beetles mating under natural conditions for 30- 
minute periods (total observation time $=699 \mathrm{~min}, \mathrm{n}=23$ pairs). They discovered that actual copulation (genital contact/intromission) accounted for less than $3 \%$ of the total observation time, the remainder of the time saw the male riding the female but without genital contact, meaning the riding behavior was not directly involved in sperm transfer. They also reported witnessing single males interfering with the paired beetles ("attacking" by running headlong into the pairs), a behavior assumed to be attempts at displacing the mounted male; no "attack" succeeded in displacing a mounted male.

In addition, over $95 \%$ of the observed pairs remained together throughout the 30 minute observation period, suggesting that $C$. marutha normally remains amplexed for a time greater than 30-minutes. Unfortunately, investigating the duration of amplexus was not part of the study and, as such, the average and range of variation of the riding behavior was left unknown. However, subsequent studies have reported riding durations for other Cicindela species ranging from 6-70 minutes (Shivashankar \& Pearson 1994, Fielding \& Knisley 1995).

The combination of 1) the minimal time spent engaged in coitus relative to the total time spent riding and 2) the witnessing of active attempted interference by unpaired males supports the supposition that the riding behavior may indeed be an evolutionarily selected response to intrasexual competition and qualify as a form of mate guarding for C. marutha. However, subsequent studies have shown that the subtleties of mating behavior differ among tiger beetle species (e.g. Shivashankar \& Pearson 1994, Fielding \& Knisley 1995, Rodriguez 1998) even if the male ubiquitously rides the female dorsally. Additionally, there are known variations in operational sex ratios, population density, and habitat complexity among the Cicindelinae, all of which may influence the strength of 
intrasexual competition, hence the value of mate guarding. Regardless, the riding behavior of all tiger beetles has been universally interpreted as a form of mate guarding over alternative explanatory hypotheses.

- Kritsky, G. \& Simon, S. (1995). Mandibular sexual dimorphism in Cicindela Linnaeus (Coleoptera: Cicindelidae). Coleopterists Bulletin, 49: 143-148.

Kritsky \& Simon (1995) represents the first formal study to address the presence and nature of sexual dimorphism within tiger beetles. By examining five species of Cicindela (each with a different type of female coupling sulcus, Freitag 1974), Kritsky \& Simon (1995) noted consistent female-biased sexual size dimorphism (based on mean body size) for all five species but variation among the species depending on whether the mandibles were longer, shorter, or similarly sized between the sexes. Four of the five species showed males having proportionally longer mandibles relative to overall body length independent of whether the mandibles were of an absolutely greater length.

A subsequent examination of only the mandibles of 31 species of Cicindela led Kristy \& Simon to define three categories of sexual dimorphism. All mandibles of the examined species share a similar morphology of a single, pronounced apical tooth subtended by three simple incisors. The identified forms of mandibular sexual dimorphism were qualitatively presented as (1) "female robust" - the females' mandibles have enlarged incisors relative to the males', (2) "dimorphic incisor"- the middle incisor of males' mandibles is modestly reduced relative to females' mandibles; and (3) 'male 
feeble" - the middle incisor of the males' mandibles is very reduced relative to females' mandibles.

Unfortunately, neither analysis involved a statistical test for significance, and only

the values of mean body length, mean mandible length, and mandible length as percent of body length were presented, without any measurement of variance. Consequently, the article was more qualitative than quantitative in nature and, lacking basic statistical analysis, unable to present anything in the way of significant trends or supportable differences.

\section{B. Study site: Powell Butte Nature Park}

\section{The park as an environment}

Field studies at Powell Butte Nature Park, Portland, Oregon, found two species of Omus: O. audouini and $O$. dejeanii. Assuming similar activity cycles, this represents a situation valuable for research, allowing a comparative study of not only sympatric but also syntopic conspecifics. Powell Butte Nature Park is a city run nature park ca. $13 \mathrm{~km}$ east of the downtown area of Portland, Oregon. The park was opened to the public in 1990 and includes 246 hectares (608 acres) of forest and meadowland over an extinct volcanic cindercone (http://www.portlandonline.com/parks/, accessed 21 Mar 2013). While the meadowland at the top of the butte had been cleared and established as a fruit orchard until 1948, there is neither mention nor evidence of similar ecological disruption occurring on the slopes and base of the butte, which remains heavily forested and includes mature Douglas-fir (Pseudotsuga menziesii) and western redcedar (Thuja plicata) (R.K.R. pers. observ.). 
The forested areas of the park extend into the surrounding non-park property and create a contiguous forest landscape of ca. 172 hectares $\left(1,724,248 \mathrm{~m}^{2}\right.$ as measured using ImageJ from aerial maps provided by Google.com, http://www.google.com map of area, accessed 21 Mar 2013; Figures $1.4 \& 1.5$ ). While the canopy is largely composed of the aforementioned Douglas-fir (closer to the top of the butte and along ridges) and redcedar (lower on the slopes and near drainages), the understory is dominated mainly by native bigleaf maples (Acer macrophyllum), vine maple (A. circinatum), red alder (Alnus rubra), and the non-native, naturalized common hawthorn (Crataegus monogyna). The understory ranges from thick to sparse and is typical of Oregon forests, with sword fern (Polystichum munitum), oregon-grape (Mahonia sp.) and salal (Gaultheria shallon) covering low-growing herbaceous plants and mosses, or forest duff and coarse woody debris. Additionally, several areas harbor groves of the invasive, non-native stinging nettle (Urtica dioica); foxglove (Digitalis purpurea) is becoming a common sight along trails.

\section{Invertebrate biota of Powell Butte and study species}

No formal arthropod surveys have been published on the invertebrate diversity of Powell Butte Nature Park. Regardless, the dense, forested habitat may be assumed to harbor an abundance of taxa representative of Pacific Northwest forest floor environments, both generalists and an occasional specialist, as well as a number of introduced non-natives as a consequence of the park's history of disturbance and proximity to an established urban environment. In my study areas at the park I have personally observed several carabid beetles typical of Pacific Northwest forest floor 
environments including species of Harpalus, Scaphinotus, Carabus, the introduced Nebria brevicollis (Kavanaugh \& LaBonte 2008), as well as other insects (e.g. other beetles, ants, true bugs, etc...), and non-insects such as myriapoda (e.g. Harpaphe harpaulus, Scolopendra sp.), arachnids (including an opilionid Taracus sp.; identity of specimen confirmed by W. Shear, Sept 2010 pers. comm..), isopods (the native Ligidium gracile and the naturalized Armadillium vulgare) and a rich diversity of microarthropods spanning several arthropod classes and orders (e.g. mites, pseudoscorpions, collembola, small rove beetles, etc...). Notable terrestrial non-arthropod invertebrates were predominately native and non-native gastropods.

The only representatives of the carabid sub-family Cicindelinae, tiger beetles, confirmed in the forested areas of Powell Butte Nature Park were two species of the genus Omus: O. audouini Reiche and $O$. dejeanii (=dejeani) Reiche, the subjects of this dissertation.

\section{Specific aims}

The two themes of this dissertation are, broadly, study of the morphology of Omus, with specific emphasis on their mandibles, and their mating behavior, primarily the duration of the riding/mate guarding stage of copulation.

\section{Ecomorphology (Chapter 2)}

Niche partioning among sympatric species can be inferred by measuring the ratios of anatomical features relevant to resource use. Using linear measurements of several anatomical features, I will examine trends in morphology between the species and 
between the sexes and ask whether they follow Hutchinsonian ratios in either body size or mandible length (as per Pearson \& Mury 1979).

Considering the importance of mandibles as organs under both natural selection for feeding and sexual selection as secondary sex organs in the males, I will specifically examine the morphology of the mandibles. To date, no study has examined morphological differences among sympatric species at the species- and sex-level or addressed how differences in mandibular shape may relate to natural prey base. With the advent of geometric morphometric techniques, morphometricians are able to examine, test and characterize differences in shape among groups in a way previously unapproachable.

Using a sample drawn from two species of sympatric Omus, I will analyze the presence and type of existing shape differences between the species and sexes. Specifically, I will ask whether there is statistically significant sexual shape dimorphism for $O$. audouini and $O$. dejeanii and determine if the character of sexual dimorphism is consistent between the species. I will also examine mandibular shape differences between the two species, seeking evidence regarding either differences or overlap of prey base for these two species of Omus.

\section{Ethogram of relevant reproductive behaviors and related observations (Chapter 3)}

While experimental practices are considered the benchmark and standard against which all research is measured, the value of non-experimental research cannot be understated, particularly when considering a poorly studied system. Observational and 
descriptive studies establish facts from which future questions may be drawn and experiments designed.

This chapter will include narrative descriptions and ethograms of courtship and mating behaviors seen in the two species being examined that were then used to design

the behavioral experiments described in Chapter 4. Similar treatments have been given to other genera of tiger beetles with patterns and variations in behavior among species and genera being identified (e.g. Rodriguez 1998, Fielding \& Knisley 1995). The addition of Omus will serve as a valuable contribution to the existing catalog of behaviors for future comparative studies.

Anecdotal descriptions will also be given related to pre-copulatory behavior (specifically the pre-copulatory struggle), same-sex pairings, and details of two witnessed events of post-copulatory intersexual cannibalism in $O$. dejeanii, an occurrence previously unreported for tiger beetles.

\section{Mating (Chapter 4)}

Female biased sexual size dimorphism is predicted to decrease in magnitude as body size increases among closely related taxa (Fairbairn 1997, Fairbairn et al. 2007). However the female must bear the weight of the male in species that obligatorily mate by the male mounting the female, producing stress for the female as well as making both individuals more vulnerable to predation and environmental stressors (Rowe 1994). This situation would presumably produce selective pressure to increase the magnitude of sexual size dimorphism, minimizing the relative size of the male, for taxa that either (1) are larger bodied or (2) engage in mating for longer durations. I hypothesize that Omus, 
being one of the larger bodied and longer mating genera of tiger beetles, will have greater sexual size dimorphism than reported for other species of tiger beetles.

Studies of mating behavior in several species of tiger beetles (all Cicindela spp.) have identified a combination of population- (operational sex-ratio and intrasexual harassment) and habitat-related (distance from suitable oviposition site) predictors governing mating duration (Shivashankar \& Pearson 1994). Predicted factors were found to more strongly influence mating duration for certain species but less so in others, suggesting that evolutionary and environmental factors influence mating durations in tiger beetles.

Despite the initial report of an amplexus exceeding 16-hours (Pratt 1939), the issue of mate guarding has not been revisited for $O$. dejeanii or for that matter, any other species of Omus. A 16-hour amplexus is an order of magnitude greater than durations reported for other tiger beetle genera, both basal and derived phylogenetically

(Shivashankar \& Pearson 1994, Fielding \& Knisley 1995, Rodriguez 1998; also see Table $1.3)$.

(1) Time of initiation of mating

With the transition between night and day there comes a dramatic change in light, humidity, and temperature, as well as a changing biotic community including a shift between visually-oriented diurnal hunters may be attracted to the increased visual profile of the mating pair versus crepuscular and nocturnal hunters that rely less on vision and more on olfactory, tactile, and auditory sensory modalities. As their common name suggests, night-stalking tiger beetles 
are presumed to have nocturnal or at least crepuscular activity patterns. Their uniformly dark coloration, in stark contrast to the patterns and hues seen in other cicindeline genera, and forest habitat suggests a limited ability to tolerate environmental stressors such as light, heat and desiccation. I hypothesize that light alone may serve as a cue and mating initiated near the onset of darkness (i.e. evening) will result in longer mating durations than those initiated shortly after the onset of light (i.e. morning). Alternatively, if there is no difference in mating duration, some other cue besides light releases mating termination behavior such as actual environmental stress (heat or disturbance) or a more cryptic cue such as communication between the participants.

\section{(2) Food deprivation}

Predacious carabid beetles, including tiger beetles, are notoriously voracious eaters, capable of consuming nearly their body weight in food daily (Thiele 1977). While a few hours of fasting may not be particularly stressful (e.g. as present in Cicindela sensu lato), mating behaviors that regularly exceed the better part of a day (or night) as expected in Omus ( $>16$ hours, Pratt 1939) may be physiologically stressful. Females, while likely handicapped, are able to forage the males are entirely precluded from eating of drinking. It is assumed that (i) mating is not a wholly fixed action pattern behavioral response and that at least one, if not both, of the participants have some degree of control over the mating duration; and (ii) the actual act of insemination requires only a small proportion of the total time observed in the mating behavior; and that (iii) insemination occurs 
at some point before the ultimate termination of contact between the partners. If these assumptions are valid, I hypothesize that food-deprived beetles will terminate mating behaviors earlier than non-food deprived beetles, disengaging to seek food upon successful insemination rather than risk personal handicap or death from starvation, added to losing the possibility of future mating opportunities.

In addition to the hypotheses proposed above, associations will be sought between mating duration and several morphological and temporal variables. Morphological features, specifically those known to be under sexual selection (e.g. female waist and male mandibles, Freitag 1974) as well as general features such as body length, will be examined as possible correlates for mating duration, with the assumption that males may invest more effort, and longer durations, in guarding larger bodied females (more fecund) than smaller bodied females in addition to a possible correlation between and anatomical features known to be under sexual selection, specifically the male mandibles and female waist.

Finally, considering the poorly known state of Omus ecology and the experimental nature of the husbandry practices for the wild-caught specimens maintained in the laboratory, time based factors of approximate age of the beetle and the duration in captivity will be addressed. Age of the beetles will be estimated by counting from the arbitrarily chosen first day of June until the day of the experiment while time in captivity will be measured in days since capture and transfer to laboratory care. 


\section{(3) Operational sex ratio}

The behavior of mate guarding explicitly assumes strong competition among males and predicts stronger expression of that behavior under male-biased sex ratios than seen in equal or female-biased sex ratios. I hypothesize that matings arranged with a male-biased operational sex ratio will endure longer than matings occurring with an equal or female-biased operational sex ratio. Because the mating behavior of tiger beetles is regarded as a form of mate guarding with copulatory (intromission and insemination) and non-copulatory stages (contact to exclude competing males) (Kraus \& Lederhouse 1983), a male-biased operational sex ratio is expected to increase the duration of the mating behavior (Parker 1970, 1974, Alcock 1994). Additionally, a female-biased sex ratio may result in shorter mating durations due to the lack of male intrasexual competition and potential for additional mating opportunities. 


\section{Chapter 2: Ecomorphology}

\section{Niches and Morphology}

\section{A. Niche Partitioning Through Character Differentiation}

Hutchinson (1957) differentiated between two subsets of the niche hypervolume: the potential conditions that could indefinitely sustain a population ("fundamental" niche, containing the entire hypervolume) and a subset within the hypervolume as the "realized" niche - those conditions (including biogeographic location) where a sustained population is actually found.

Assuming finite access to resources among species, overlapping needs often results in one species outcompeting and displacing another (although complete extirpation appears to be rare: Connell 1983 and Schoener 1983). Overlapping realized niches might develop into adjacent realized niches as populations partition the environmental resources (Schoener 1974), particularly within low diversity communities (Connell 1980). Importantly, the functional niche may remain unaffected and resource partitioning among competitors due to non-heritable mechanisms, such as learned behaviors, differential gene expression during development or competitive exclusion. Alternatively, the functional niche of the population may be affected through heritable changes in phenotype — evolutionary adaptation — and cause narrowing of the species' niche and ecological specialization (Futuyma \& Moreno 1988). This ecolological mutual exclusion may be paraphrased in "Gause's axiom": no two species can occupy the same ecological space (Gause 1934, Vandermeer 1972).

Extending beyond competition for food, resources may be any factor essential to the biology of the organism in question, including nutrition, mate-display sites, 
ovipositioning sites, time of day (Kronfeld-Schor \& Dayan 2003), micro-habitats serving as refugia against environmental physiological stressors or predation, or even overlapping communication frequencies (in bats: Aldridge \& Rautenbach 1987, Kingston \& Rossiter 2004, Siemers \& Schnitzler 2004; in cicadas: Sueur 2002).

The presence of competition within a niche can be inferred by comparing characters related to exploiting the resouce in question, e.g., ovipositor structure for brood sites or mouthparts for food sources. Hutchinson (1959) proposed that differences in body size along a "critical" threshold factor of ca. x1.3 (e.g. $10 \mathrm{~mm}, 13 \mathrm{~mm}, 17 \mathrm{~mm}$, etc...) allowed resource partitioning and coexistance among otherwise potential competitors. These "Hutchinsonian ratios" are often used as evidence of adaptation and specialization in response to past competition pressure and popularized to the point of being regarded as a general ecological rule but, upon examination, are not without exceptions (Simberloff \& Boecklen 1981). Niche partitioning solely through body size differentiation along Hutchinsonian ratios assumes that resource use is correlated with body size, and has been widely observed among predators under the circumstances of broad prey size-class distributions (Wilson 1975). Additionally, character that do not scale isometrically with body size and form an allometric relationship can identify traits that are under special selective pressures or restrictions (Gould 1966, 1974) giving further evidence to hypothese related to character divergance.

\section{B. External anatomy of insects}

1. Insects in general 
As arthropods, insects bear an exoskeleton that is periodically molted between developmental or growth stages. In contrast to the soft superficial tissue layers of most vertebrates and non-arthropod invertebrates, the exoskeleton forms discrete, rigid structures well suited to morphological analysis. The exoskeleton, also termed the cuticle, is composed of layers of polysaccharide (chitin) embedded within a protein matrix (Triplehorn \& Johnson 2005). The precise chemical composition of the cuticle varies with the species, the individual and even the condition of the individual and carries complex hydrocarbon compounds used in recognition and signaling (Singer 1998). The cuticle is resistant but flexible unless treated; the majority of the larval (immature) cuticle is unsclerotized making the early stages of development particularly prone to parasitism and dessication. Insect development includes processes to differentially harden, or sclerotize - a process not unlike tanning leather, specific regions of the cuticle and form plates generally called sclerites. Sclerites forming the dorsal aspects of the exoskeleton are specifically tergites while those plates forming the ventral aspect of the insect are sternites. Surfaces in between sclerites, such as joints, remain unsclerotized and flexible for movement or expansion. Additional compounds such as waxes may be secreted to prevent dessication or abrasion. Perforating the lateral walls of the thorax and abdomen are a series of minute holes called spiracles that allow for gas exchange in the absence of lungs. Far from being an insensible "suit of armor" the insect exoskeleton is saturated with sensory organs including external setae, chemoreceptors and internal strain receptors allowing subtle detection of the mechanical and chemical environment (Klowden 2007).

The class insecta shows vast diversity of form but maintains a basic, wellconserved adult body plan consisting of (1) an anteriorially located head, (2) a thorax and 
(3) a posteriorally located abdomen. The head bears feeding appendages - often highly specialized — and sensory organs such as compound eyes, light-detecting occili, setae and antennae. Internally, the head contains the brain and the anterior part of the alimentary canal. The thorax bears legs and, when present, wings. Interally, the thorax contains the heart, the midgut and conducts the verntral nerve cord from the brain to the abdomen. Superficially, the abdomen is relatively simple compared to the head and thorax and bears few external structures aside from repeating, sclerotized plates separated by flexible, non-sclerotized cuticle allowing for flexibility and expansion. Internally, the abdomen contains the reproductive organs, terminal portions of the alimentary canal and the excretory system.

As adults, all insects bear three pairs of legs: forelegs, midlegs and hindlegs. Certain taxa show reduction of the legs and other external features particulary among endoparasitic species (e.g. twist-winged parasites, Order Strepsiptera). The legs are multi-segmented structures that follow a similar pattern but may be highly adapted and specialized as with the raptoral forelegs of mantids (Order Mantodea), waterscorpions (Hemiptera: Nepidae) and ambush bugs (Hemiptera: Reduviidae) (Triplehorn \& Johnson 2005). The legs extend from the ventral surface of the thorax and consist of four distinct structures (described proximally to distally): (1) a small, proximally located coxa, (2) a small trochanter, (3) the femur - the first long segment, (4) the tibia - the second long segment, (5) a series of small segments that together form the tarsus that terminates into (6) a pretarsus consisting of claws, pads or modifided setae (Triplehorn \& Johnson 2005).

\section{Beetle body plan}


Beetles (Order Coleoptera) are a highly diverse group of insects with many specific adaptations allowing them to exploit a wide variety of resources. Regardless, all beetles share certain taxonomically defining anatomical features. On the head, all beetles bear mandibulate mouthparts consisting of a pair of hardened, chewing appendages. Mandibles bracket the oral cavity and articulate along a lateral-medial axis, allowing food to be processed for ingestion. Many beetles, particularly predaceous types, use extra-oral digestion using the mandibles to grind food (the "oral mill") while secreting digestive enzymes and then drawing the fluid into the oral cavity rather than ingesting solid, particulate food (Cohen 1995).

Beetles possess two pairs of wings, the forewings and the hindwings. The forewings are hardened into protective sheaths called elytra that cover the hindwings. Among certain taxa (e.g., several members in the Family Carabidae), the elytra have fused and the hindwings reduced (brachypterous) or entirely lost (apterous) rendering those beetles incapable of flight (Thiele 1977, Lovei \& Sunderland 1996). The two elytra, fused or unfused, align parallel to each other and form a conspicuous elytral suture running down the center of the dorsum of the abdomen. This medial elytral suture serves as a useful diagnostic differentiating Coleoptera from Hemiptera (true bugs), which possess a similar hardened wing case but overlap rather than run parallel.

\section{Tiger beetle body plan}

Tiger beetles are all morphologically similar; the bodyplan being distinctive and well conserved throughout the subfamily. The individual body segments are well defined. The head bears large compound eyes, conspicuous, sickle-shaped mandibles and 
long, filiform (thread-like) antennae. Most tiger beetles are capable of flight however they primarily cursorial hunters, chasing prey on long, thin legs. Basal members such as Omus and Amblycheila have fused elytra and reduced or absent hindwings making them incapable of flight. A single, large sclerite, the pronotum, covers the majority of the dorsal surface of the thorax.

The cuticle of tiger beetles is a complex multilayered organ of not only chitin and protein but also waxes and pigment molecules. The microanatomy and composition of the cuticle determines the coloration of the beetle and many species are showy and brightly colored with contrasting patterns and metallic tones. Disorganized arrangement of layers and pigment compounds results in dark, uniform coloration seen in genera like Omus and Amblycheila.

\section{Morphometric analysis of linear dimensions: comparison of species and sexes A. Introduction}

Morphology is the consequence of two characters: shape and size. Drawing from Gould (1966), allometry is the examination of proportional differences in shape relative to changes in the magnitude of overall size: as overall size changes (by physiological development of an individual or evolutionarily among taxa), anatomical proportions may or may not remain constant. A fundamental assumption governing modern theories of evolution is that biological similarity is a function of evolutionary relatedness: the more closely related are two species, the more similarities they will likely share. As such, congeneric species living in sympatry are expected to have shared needs and will likely experience competition unless adapted to differentially exploit available resources, i.e., 
“Gause's axiom": no two species can occupy the same ecological space (Vandermeer 1972). Therefore, sympatric congeners are expected to show differences precisely to minimize the competition predicted due to their shared evolutionary history, the differences possibly even exacerbated where there is high probability of contact as a form of character displacement (Brown \& Wilson 1956, Dayan \& Simberloff 2005). Allometric trends among taxa (or developmental stages within a single species) and simple morphological differences among closely related species can inform us of differences in ecological resource use as well as genetic constraints limiting morphological differentiation. Hindleg muscle mass, direct measurements of grip strength and talon curvature among raptors relates to prey base and hunting behaviors (Ward et al. 2002, Fowler et al. 2009); surface area and lenses of the compound eyes of Carabid beetles have been related to preferred habitats and activity cycles (Talarico et al. 2007).

The often sympatric (occurring in the same habitat), and likely syntopic (sharing similar activity pattern), tiger beetles Omus audouini and $O$. dejeanii are representatives of a genus of alleged low taxonomic diversity (ca. five recognized species) and assumed to have relatively non-specific predatory habits (van den Berghe 1990, Pearson \& Vogler 2001, Pearson et al. 2006). While overall body size differs between these two species, body size alone is rarely justification for evidence of niche partitioning, excepting interactions among top predators within an ecosystem (Wilson 1975). More likely, allometric or other subtle differences in anatomy in conjunction with body size determine differences in ecological resource use. 
I sought to determine whether these two beetle species $O$. audouini and $O$.

dejeanii exhibit differences in overall anatomical proportions, implying novel adaptations resulting in niche partitioning, or Hutchinsonian ratios in body size and mandible length. Both body length and mandible length have been associated with prey handling ability among predaceous species, specificially tiger beetles (Pearson \& Mury 1979) and regarded as valuable characters to infer resource use, specifically prey base.

Considering the evidence of sexual size dimorphism occurring within the Cicindelinae, proportional differences between the sexes as well as species were compared using a combination of features known to be under sexual selection (mandibles, waist width, etc...) and characters not under the influence of sexual selection but maybe subject to other selective pressures specific to resource use and habitat (leg length, body width, etc...).

\section{B. Materials and methods for allometric comparison of species and sexes}

To address questions of ecomorphological differences between species and trends of sexual dimorphism within and between species, I used a dataset of 233 observations collected over two years (2009 \& 2010) representing four classes: Omus audouini female $(\mathrm{n}=70)$, O. audouini male $(\mathrm{n}=70)$, O. dejeanii female $(\mathrm{n}=43)$, and $O$. dejeanii $(\mathrm{n}=50)$. I measured for 11 morphological features related to overall body morphology on all specimens. The gross morphological characters are illustrated in Figure 2.1 and described in Table 2.1. All distances were direct linear (Euclidian) measurements between landmarks rather than following the curvature of the body. All measurements were taken by hand with a pair of either SPI 6"/150 mm dial calipers (model \#31-415-3, 
dial graduations: 0.1 mm; Swiss Precision Instruments, Inc., P.O. Box 3135, Garden Grove, CA 92842, USA; www.swissprec.com) or Mitutoyo 4"/100 mm dial calipers (model\# 505-636-50; dial graduations: 0.05 mm; Mitutoyo America Corporation, 965 Corporate Blvd., Aurora, IL 60502, USA; www.mitutoyo.com). Specimens were measured while viewed under a dissecting microscope as needed. Due to flexion and compression at points of articulation, body length was reported to the nearest $0.5 \mathrm{~mm}$, antenna length was rounded up to the nearest $0.2 \mathrm{~mm}$ and femur length was measured to the nearest $0.1 \mathrm{~mm}$. While the tibia was a single structure without a joint, the distal margin of the tibia has prominent setae that may have added to the imprecision of this measurement and was measured to the nearest $0.1 \mathrm{~mm}$. All other measurements (waist width, mandible length and head width) did not involve jointed or otherwise confounding features and were taken to the nearest $0.05 \mathrm{~mm}$. Only specimens able to provide the full suite of measurements were included for morphological analysis. Principal component analysis of the resulting measurements (R, "prcomp") was done to reduce the number of variables, maximize individual variation, and visually identify trends (Bookstein et al. 1985, Zelditch et al. 2004, Slice 2007) within and between the species. A Ryan-EinotGabriel-Welsch multiple range Q-test for variance (option means/REGWQ in SAS' PROC GLM, a multi-class ANOVA; Day \& Quinn 1989) was used as a univariate test for significant differences among groups. Analyses were performed using R, version 2.15.2 and SAS ${ }^{\circledR}$ statistical software, version 4.2.

\section{Results for comparison of species and sexes}


Summary statistics for individual characers are given in Table 2.2. A REGWQ multirange test for significant differences among the invidiual characters (Figure 2.2) shows $O$. dejeanii are significantly larger than $O$. audouini in all characters. Females of both species are significantly different $(\alpha=0.05)$, specifically larger-female biased sexual size dimorphism, than males for most characters. There was no sexual dimorphism in absolute length of antennae for either species and, while $O$. audouini show sexual dimorphism in mandible length, these was no sexual dimorphism in mandible length for $O$. dejeanii.

Reduced major axis regression of $\ln$ (antenna length) onto $\ln$ (body length) produced a significant $(\mathrm{p}$-value $<0.02)$ but non-linear $\left(\mathrm{r}^{2}<0.50\right)$ relationship for all categories (Table 2.6A). Reduced major axis regression of $\ln$ (mandible length) onto $\ln$ (body length) was also significant ( $\mathrm{p}$-value $<0.001)$ and non-linear $\left(\mathrm{r}^{2}<0.40\right)$ for all categories. Mandibles for male O. audouini scaled hyperallometrically $(\beta=1.44)$ indicating that mandible length increases proportionally faster than body length but not linearly $\left(r^{2}=0.29\right)$.

The body lengths and mandible lengths for the sexes within each species were averaged and the proportional differences were compared. The body lengths between the two species $(O$. audouini/O. dejeanii) were x1.3 while mandible lengths were x1.5 (Table 2.3 A), both values meeting or exceeding Hutchinsonian ratios predicting niche partitioning among sympatric species. Evidence for sexual niche partitioning was less convincing than interspecific partitioning as proportional body length and mandible length for $O$. audouini $(\mathrm{M} / \mathrm{F})$ was 1.1 for both and $O$. dejeanii $(\mathrm{M} / \mathrm{F}) 1.1$ for body length and 1.0 for mandible length (Table 2.3 B \& C). 
The principal component analysis including both species clearly separates individuals belonging to the four a priori designated categories into distinct groupings (Figure 2.3). Table 2.4 gives the character eigenvectors and principle component eigenvalues for the species comparison PCA. The eigenvectors of the first principal components axis (PC1) are all of the same sign and similar magnitude, indicating that PC1 may be regarded as a general "size" axis: increasing values along PC1 reflect a generally consistent increase in overall size and clearly separates the two species.

The principal component analyses of the individual species separate the sexes into distinct groupings with similar Eigenvectors as seen in the species comparison PCA (Figure 2.4, Table 2.5). The exception between the species is the value of mandible length in determining sex: positive travel along PC1 decreases all body measurements except mandible length for $O$. audouini while all positive travel along PC1 decreases all body measurements including mandible length.

\section{Discussion for comparison of species and sexes}

Average body length between the two species showed $O$. dejeanii was 1.3 times larger than $O$. audouini while average mandible length for $O$. dejeanii was 1.5 times larger than $O$. audouini. Both body size and mandible length are associated with prey base through prey handling ability. The scaling factor of $\geq x 1.3$ supports the notion that these two species exploit different prey bases but does not exclude the possibility of occasional resource compatition through prey overlap or intraguild predation. Niche partitioning between sexes is known for certain animal species exhibiting pronounced dimorphism, however, it is believed that the resource differentiation (trophic 
dimorphism) developed secondarily to pre-existing sexually dimorphic traits rather than intrinsic radical differences in the needs of the sexes preceding sexual dimorphism (Fairbairn 1997). However, there was little evidence to suggest sexual niche partitioning between the sexes for either species; body length and mandible length ratios were $\leq 1.1$ between the sexes.

Female-biased sexual dimorphism was present for almost all characters and can be interpreted as a consequence of increased overall body size between the sexes. Three exceptions to female biased sexual sized imorphism in specific characters were (1) the antenna of both species, which did not show significant differences in length, (2) the mandibles of O. audouini, which were significantly male-baised in length and (3) the mandibles of O. dejeanii, which were not significantly sexually dimorphic in length (Figure 2.2).

The lack of sexual dimorphsm in antenna length despite body size differences suggests a functional constraint on the absolute length of the antenna in Omus rather than a simple relationship scaling with body size or discretely with sex. All Cicindelines have simple filiform (threadlike) antennae made of eleven segments and are not reported to rely on pheromones for intersexual attraction or communication. It is not known how the specific anatomy of the antenna is different between the sexes, whether the male antennae are proportionally more slender than female antennae or if they are the same length and width. If the antennae are the same in both length and width for both sexes, it suggests an anatomical constraint related to the functioning of the organ, possibly a limit to the number and diameter of neurons or density of receptors able to fit in an antenna. 
If the male antennae are more slender than female antennae, then absolute length is the important factor rather than a functional constraint of anatomy. The length of the antenna could possibly under sexual selection related to syn-copulatory courtship behaviors reported among beetles in genereal including several genera of tiger beetles: the mounted male strokes and taps the head and antenna of the female with his own antennae (Willis 1967, Thornhill \& Alcock 1983, Eberhard 1994, Fielding \& Knisley 1995, Rodriguez 1998). As such, antennae of specific minimum length would be necessary to adequately contact and communicate with the female while mounted.

Principal componant analysis describes these two species of beetle as essentially scaled versions of the same general form with general, archetypal female and male forms. When the species are examined individually, the sexes differentate in similar ways both both species, mainly by a similar size factors. An exception is in the trait of mandible length, where male $O$. audouini decrease in all body characters except mandible length which increases relative to female $O$. audouini. This relationship is not seen in $O$. dejeanii: males decrease in all characters relative to females.

Broadly, a female Omus will have a larger body (larger thorax and elytra along both length and width), but proportionally shorter antennae and mandibles, in contrast to a typical male form that would present a smaller body but proportionally enlarged antennae and mandibles.

Broad morphological and allometric trends have been valuable in determining how an animal may use available resources. Here, it has been demonstrated that body size alone is suggestive of niche partitioning between the species, likely through exploitation of prey along a size gradient. We also see evidence suggestive of a 
previously unaddressed sexually selected character of the length of the antennae. While these results alone are not robust enough to be the definitive answer, they identify potentially fruitful directions in integrated behavioral, fine-scale morphological and natural observational studies to reveal how these animals relate to their environment.

\section{III: Geometric morphometric analysis of mandibles}

\section{A. Introduction}

Insect mouthparts show great diversity in form and function. Coleoptera, while limited to mandibulate mouthparts, exhibit occasionally extreme morphological adaptations due to feeding behaviors, ecologies and sexual selection. The diversity of diet among beetles has necessitated the need to accommodate a variety of foods in terms of nutritional value and hardness. In addition to microstructural adaptations including the incorporation of metals, predominately zinc, and crosslinking of cuticular proteins to dramatically increase the hardness of the mandibles (Cribb et al. 2008), morphological adaptations are well know and account for the variety of shearing, piercing and crushing motions required to process food (Acorn \& Ball 1991). More than simply tools for ingestion, the mandibles of beetles are essential in allowing the animal to manipulate its environment through various activities including, excavating substrate (e.g. bark beetles, particularly of the genus $I p s$ ), defense against predators and grooming. Mandibles extend to further functions and can even serve as secondary sexual organs under strong sexual selection involved with female choice as well as weapons in male-male competition (Emlen et al. 2005). 
The carabid beetle subfamily Cicindelinae, the tiger beetles, are predaceous both as larvae and adults, and universally exhibit long, sickle-shaped mandibles (Pearson \& Vogler 2001) that they use to capture and process prey items in preparation for extra-oral digestion (Evans 1965). As organs of prey capture and defense, the adults' mandibles serve to capture, pierce, shear, and masticate invertebrates of nearly the same body size, and likewise effectively discourage unwanted contact by other animals (R.K.R. pers. observ.).

As secondary sexual organs, males use their mandibles to grasp females around the "waist," the constriction between the thorax and abdomen. The mandibles contact the mesepisterna, a pair of plates that form the ventro-lateral aspect of the waist. Once mounted, males maintain extended paired contact presumed to be a form of mate guarding both during coitus and non-coital periods (Pratt 1939, Freitag 1974, Kraus \& Lederhouse 1983). The relationship between male mandibles and female waist has been strongly shaped by sexual selection such that females of several cicindeline genera (e.g. Cincindela sensu lato) possess species-specific coupling sulci on their mesepisternum that accept the tooth apices of the males' mandibles (Freitag 1974).

Male-biased sexual size dimorphism of the mandibles, either in proportion to body size or in absolute length, is known in many species of cicindelines as measured by mandibular chord length, i.e., the direct Euclidian distance from the point of articulation to the distal point of the apical tooth of the mandible (Pearson 1980, Kritksy \& Simon 1995). Despite the crucial role of these organs in the ecology and life history of tiger beetles, intersexual mandibular shape differences, or sexual shape dimorphism (as opposed to only size) have remained largely unexplored. 
Having to serve the dual roles of feeding organs and secondary sexual organs, tiger beetle mandibles need to be either morphologically generalized enough to adequately function in both capacities or be specialized such that different regions of the mandibles are optimized for specific roles or functions. To further complicate matters, the additional role of secondary sexual organ is exclusive to the male. Thus, development must modify a pre-existing genetic template along a sex-defined trajectory rather than a standard, multi-use tool available to both sexes.

For males, there is the need for a compromise between a minimum structural strength insuring adequate structural integrity for prey capture and processing, and the specific length and curvature required to adequately secure the female waist during reproduction. Furthermore, since prey-capture and processing ability would be largely influenced by the size and shape of the mandibles, mandibular morphology also sets limits on the potential prey base (functional niche, sensu Hutchinson 1957). Consequently, any variation in shape may have dire consequences in terms of performance - either in feeding or mating - and, hence, fitness. Conceivibly, sexual shape dimorphism in anatomy such as the mandibles may generate selective pressure that result in the divergence of prey base between the sexes.

Mandibular morphology, in terms of length and bite gape, has been associated with the ecological niche because of the tight, size-based linkage to prey base among sympatric species of tiger beetles (Pearson \& Mury 1979). 
All other things being equal, an arthropod's resistance to penetrating, shearing, and crushing forces would increase with body size as the cuticle would also increase. While larger invertebrates become more nutritionally "profitable" because of their greater mass, they also become more difficult to process for ingestion and require alternative strategies to exploit. Despite the importance of the mandibles in mating and prey acquisition behaviors, there has been no treatment on how the shape of the mandible, rather than mere linear size, may change among species to account for these mechanical challenges.

Mandibular sexual dimorphism in tiger beetles, as opposed to mandibular morphological diversity among species, was briefly addressed by Kritsky \& Simon (1995) but aside from length and qualitative terms, the issue of precisely how mandible shape differs between the sexes, not to mention among species, has not been approached. I hypothesized not only that males and females will have differently shaped mandibles but also, due to the similar mating behaviors, that these shape differences would be consistent across species.

Night stalking tiger beetles, Omus, represent evolutionarily basal members of the tiger beetles (Vogler \& Pearson 1997). In addition to being relatively large bodied, they are flightless, reasonably easy to maintain in a laboratory setting, and are readily collected using pitfall traps - all characters enhancing their suitability for study. Despite these advantages, Omus remain among the least studied genera of tiger beetles. As such, the present study serves the dual purpose of examining patterns of geometric morphology of a predaceous terrestrial beetle species' mandibles, as well as advancing our knowledge of the genus Omus. 
Two species of sympatric, and likely syntopic, night stalking tiger beetle, $O$. audouini and $O$. dejeanii, were used to address the issue of intersexual and interspecific differences in mandibles by comparing among four a priori groups: $O$. audouini female, O. audouini male, $O$. dejeanii female, and $O$. dejeanii male. Shape differences were described, quantified and tested for significance. Specifically, I tested the hypothesis of mandibular isomorphy, i.e., that the mandibles are the same shape and only size differences are present among the groups with significant differences identifying morphological features due to sexual selection or ecological segregation. The alternative hypothesis is that identifiable shape differences exist that are specific to the demands of that category, either by sex (sexual selection) or by species (natural selection adaptation for prey).

\section{B. Materials and methods}

\section{Specimen information}

Night stalking tiger beetles (Omus) are endemic to the west coast of North America west of the Cascade mountain range, and form a complex of at least five recognized species with several regional varieties (Freitag 1999). Omus are flightless and found primarily in densely sheltered forest and meadow habitats, in contrast to the more derived cicindeline genera which, characteristically, are aggressive fliers found in exposed habitats such as sandy areas, ashflows, stream banks, and salt flats (Knisley \& Schultz 1997, Pearson \& Vogler 2001). The two species of this study, O. audouini and O. dejeanii, are exclusive to Washington and Oregon and are often found sympatrically 
and likely syntopically as well. However, O. dejeanii appears to be more restricted to deep forest habitats than O. audouini (Maser 1977a \& b; van den Berghe 1990).

I examined a total of 110 individual specimens: O. audouini $=66$ (female $=34$, male $=32)$ and $O$. dejeanii $=44($ female $=22$, male $=22)$. All specimens were collected by pitfall trapping during the spring and summer months of 2010 and 2012 from the forested areas of Powell Butte Nature Park (ca. 45²9'14.5” N, 122³0'06.5” W), a cityrun park ca. $13 \mathrm{~km}$ east of downtown Portland, Oregon, USA (Figure 1.5). Specimens were stored in $70 \%$ alcohol (either ethanol or isopropyl) pending examination.

\section{Linear measurements}

I used linear measurements to provide an estimate of specimen body size and mandible length. Size measurements (as length as well as higher dimensional measures such as area and volume) are valuable for determining whether shape variation scales with the size of the individual (or organ). In that manner, shape variation is explicable as a consequence of growth. Alternatively, shape may be decoupled from simple growth and illustrate shape differences as consequences of natural or sexual selective pressures (e.g. Gould 1966 \& 1974, Andersson 1994).

In addition to body size, mandible length is among the more common and valuable measurements used in studies of tiger beetle ecology because of its relationship with the ecological niche (more specifically prey base) among sympatrically occurring species (Pearson \& Mury 1979).

Direct body length (linear distance from caudal to cephalic ends) was an inconsistent measure because of the compression of joints and occasional damaged 
specimen. Instead of body length, the summed lengths of the pronotum and elytra was used (Figure 2.5), giving greater repeatability to measurements and, also, could be used on broken specimens.

Mandible length was measured using the digital images of disarticulated mandibles produced during the geometric morphometric analysis using the software ImageJ (Rasband, W.S., ImageJ, U. S. National Institutes of Health, Bethesda, Maryland, USA, http://imagej.nih.gov/ij/, 1997-2012) and size calibrated with a $5 \mathrm{~mm}$ scale situated on the same focal plane. Mandible length was defined as the linear distance between the distal point of the apical tooth and the point immediately distal to the articulating hinge along the lateral margin of the mandible (landmarks \#1 and \#8; Table 2.7, Figures 2.5, $2.6 \& 2.7)$.

\section{Methods of geometric morphometrics}

Fundamentally, the methods of landmark-based geometric morphometrics reduce a specimen into a series of discrete landmarks represented by Cartesian coordinates. The coordinates themselves may be generally thought of as the corners of a polygon which may be compared to other specimens, also reduced to a series of landmarks representing a polygon. However, the use of the term "polygon" is only an analogy because, using landmarks rather than lines, we lack information regarding the spaces between the landmarks. As such, it is more correct and less misleading to properly refer to the data as an arrangement of landmarks.

Morphology refers to both the component of size and that of shape. The advantage of geometric morphometrics lies in the technique's ability to directly compare 
shapes, independent of non-shape components. Non-shape components include size (the other component of morphology) as well as the non-morphological components of position and rotation, artifacts introduced by converting the specimen to Cartesian coordinates. To remove the component of size, the arrangement is scaled to unit centroid size (divided by the square root of the sum of differences among the landmarks, Equation 2.1).

$$
C S=\sqrt{\sum_{i=1}^{n}\left[\left(x_{i}-\bar{x}\right)^{2}+\left(y_{i}-\bar{y}\right)^{2}\right]}
$$

Where:

$$
\begin{aligned}
& \mathrm{x}_{\mathrm{i}}=\mathrm{x} \text {-coordinate of landmark } i, \bar{x}=\mathrm{x} \text {-coordinate for configuration centroid } \\
& \mathrm{y}_{\mathrm{i}}=\mathrm{y} \text {-coordinate of landmark } i, \bar{y}=\mathrm{y} \text {-coordinate for configuration centroid }
\end{aligned}
$$

The centroid size (CS) itself may be used as a general index of size for comparing among individuals and useful for allometric regression analyses. Centroid size is a preferable measurement of "size" compared to standard geometric area calculations because area depends on the area enclosed by a shape; however, as mentioned, we only have information regarding points, not necessarily the space between the points. Centroid size is preferable becuase it measures the mean distances of the points from a central (average) point and thus avoids the issue of assuming unknown information.

Once the arrangement is scaled to unit centroid size, standard methods of geometric translation are used to centrally align and rotate the configurations to minimize positional differences among corresponding landmarks. This process of scaling, aligning, 
and rotating an arrangement of landmarks is the General Procrustes Alignment (GPA; also General Procrustes Superimposition, GPS) method of superimposition, one of several proposed methods of minimizing differences among landmark arrangements for subsequent comparison (Adams et al. 2004, Zelditch et al. 2004, Slice 2007; see references for alternative alignment/superimposition methods).

The resulting aligned coordinate arrangements may then be tested for statistical differences through specialized multivariate analysis of variance (MANOVA) analogs. A noteworthy consequence of the superimposition is a loss of several degrees of freedom. In the case of two-dimensional configurations, degrees of freedom are lost from scaling, alignment along each axis, and rotation, resulting in $2 \mathrm{~K}-4$ degrees of freedom where $\mathrm{K}$ is the number of discrete landmarks for an analysis in two dimensions. Due to this factor, the more common statistical tests - typically based on degrees of freedom equal to n- 1 are inappropriate for direct coordinate data. Goodall's F-test (a MANOVA) and Hotelling's t-test are specifically adapted for GPA coordinate data accounting for the decreased degrees of freedom and can be used directly on the superimposed coordinate to test for significant differences among mean shapes of groups. Discussions of shape space (e.g. Bookstein 1986, Rohlf 1999, Slice 2001), consequences of projecting specimen positions within non-Euclidian shape space on a tangential Euclidian plane, and use of Procrustes distance among specimens for analysis will not be addressed here.

Beyond specifically extracting non-shape factors from shape for morphological analysis, an additional strength of geometric morphometric techniques is that it allows changes in shape to be visualized across the entire form using a mathematical thin-plate spline function (Bookstein 1989, Zelditch et al. 2004). Thin-plate splines are used to 
represent differences among the landmark arrangements for each specimen, modeling the change as a deformation of an otherwise orthogonal grid. The "reference" form, represented by the undeformed, orthogonal grid, may be a specific designated individual to which all others are compared or, more commonly, the undeformed grid represents an artificially constructed "consensus" arrangement - an average of all of the specimens included in the study. An advantage of using the thin-plate spline function to model shape change is that it allows inferences regarding the shape changes happening between the landmarks in contrast to the methods previously introduced where the space between the landmarks was wholly unknown.

The deformations shown by the thin-plate spline function may be either uniform (or "affine") and represent a global shape change (either shearing or a non-scaled compression/dilation) or may be nonuniform ("nonaffine") where the deformation is localized to a specific region of the larger configuration. Partial Warp Scores (PWS) are coefficients of the thin-plate splines and can be used as the functional dataset for describing the nonuniform components of deformation.

A Relative Warp Analysis (RWA), analogous to a principal components analysis (PCA) of the PWS (Rohlf \& Bookstein 2003, Adams et al. 2004, Slice 2007), can be performed to decompose the set of partial warp scores into a series of independent deformations (Principal Warps) accounting for the observed morphological variation. As with a PCA, the axes of a RWA describe the magnitude of the independent deformation. These deformations can be graphically represented through thin-plate splines (as wireframe figures) showing the changes involved in transitioning from a reference configuration to a target configuration. Typically, an artificially constructed average 
"consensus" configuration of the groups is used as the default reference (represented as the origin of the RWA plot) with the target configuration being an individual specimen or arbitrary position on the RWA (e.g. the centroid of an a priori group). Unlike the superimposed shape coordinates, the RWA scores do not have decreased degrees of freedom and can be analyzed using conventional statistical tests.

In contrast to discrete landmarks, regions lacking consistently identifiable points (e.g., a curve) may be described though sliding/semi-landmarks. These may be thought of as a relaxation of the requirement of one-to-one homologous correspondence for discrete landmarks but carry a necessary relaxation of inferred biological significance of any single point along the curve. In the case of a curve, a series of semi-landmarks are placed along the perimeter and a mathematical function is fitted to that configuration. When the resulting configurations are subjected to superimposition, the rigidity of location of the semi-landmarks relative to other landmarks is relaxed allowing those semi-landmarks to "slide" along the curve as needed to facilitate a superimposition of minimum difference with the other individuals. Once superimposed, differences between shapes may be described in terms of landmark displacements and visualized as deformations from a reference configuration to a target configuration.

\section{Geometric morphometric analysis of mandibles}

The left mandibles were removed from alcohol-preserved specimens and dorsal aspects digitally photographed with a Leica MZ12.5 stereomicroscope using Leica IM50 imaging software (version 5, release 247). A 5mm scale (Minitool, ST-027; Minitool, Inc., 634 University Ave, Los Gatos, CA 95032) was included in all photographs for 
calibration. All specimens were imaged in a standard orientation and only mandibles in good condition and without excessive signs of wear or damage were used. The incisor was broken from the mandible of a single specimen during the disarticulation process. The break was clean enough that the two pieces could be photographed and the entire mandible digitally reconstructed by matching the ends of the pieces.

The initial datafile generation, landmark placement, and general Procrustes alignment were performed using the tps suite of software (tpsDig2, Rohlf 2010a; tpsrelw, Rohlf 2010b; tpsutil, Rohlf 2012; available at http://life.bio.sunysb.edu/morph/). Eight landmarks were used to define the apices and sulci of the medial margin of the mandible anterior to the molar complex and the point anterior to the articulating hinge on the lateral margin of the mandible (Figures 2.6 \& 2.7; landmarks defined in Table 2.7, terminology follows Ball et al. 2011 when able).

Ten sliding landmarks (also known as semi-landmarks) were used to define the outer (lateral) curve of the mandible. The sliding landmarks were defined by placing 2030 points along the margin of the curve using landmarks 1 and 8 as the proximal and distal end points. The curve was then resampled to 12 points evenly distributed along the path. The landmark data file was edited in the program tpsUtil to remove the curve end points, as they were redundant with landmarks 1 and 8 , resulting in a total of ten semilandmarks. The curve was then converted to landmarks and a slider file generated in tpsUtil (Rohlf 2012). The resulting configurations were subjected to Generalized Procrustes Alignment (GPA) to remove non-shape characters of position, rotation, and scaling (Rohlf 1999). In addition to aligning landmark configurations for comparison, a consequence of GPA is the calculation of the centroid sizes of the individual 
configurations. The centroid size of a configuration is the square root of the sum of squared distances of the landmarks from their centroid and serves as a convenient and accepted measure of the size of a shape in geometric morphometric analyses (Bookstein 1996, Slice et al. 1996, Frost et al. 2003)

\section{Shape analysis of mandibles, angular measurements and curvature}

Differences in mandibular shape among the aligned coordinates were visualized using a thin-plate spline function and the shape differences were compared to an averaged form, the "consensus shape" (Bookstein 1989). Relative warps analysis (tpsRelw v1.49, Rohlf 2010b), a procedure analogous to a PCA of the aligned coordinates but using variables weighted by bending energy of the thin-plate spline function (Zelditch et al. 2004), was used to explore grouping patterns of the individuals in relation to the described shapes (Rohlf \& Marcus 1993).

Thin-plate spline visualization can reveal localized shape variation but does not provide quantification of specific details. I measured the angles of the terebral teeth (i.e. the two "inner" teeth between the proximal "molar" complex and the distally located apical incisor; see Figures $2.6 \& 2.7$ ) and the curvature of the apical incisor, the features best represented by the landmarks used in the geometric morphometric analysis. I used the Procrustes aligned landmark coordinate data from the geometric morphometric analysis to calculate and so was able to compare measurements independently of scale.

The angles of the terebral teeth relative to the point of articulation were measured using the distal base of tooth as the vertex and the tip of the tooth and the point of articulation as the endpoints. Using the coordinate data for the three points as three 
points of a triangle (landmarks 1, 5, 4 for the proximal terebral tooth and 1, 7, 6 for the distal terebral tooth; Fig 2.7), the geometric distance formula, i.e.

$d=\sqrt{\left(x_{2}-x_{1}\right)^{2}+\left(y_{2}-y_{1}\right)^{2}}$, was used to calculate the length of the sides, and the trigonometric law of cosines - relating the lengths of two sides of a triangle to the cosine of an angle - used to calculate the desired angle.

The curvature of the apical incisor was described with the coordinates used to define landmarks \#9-13 (corresponding to the outer, or lateral, curvature of the tooth) from the geometric morphometric analysis. Using the curve fitting function in ImageJ the landmarks were used as points that were fitted to a 2 nd degree polynomial in the form of:

(Eq 2.2) $\quad \mathrm{y}=\mathrm{A}+\mathrm{Bx}+\mathrm{Cx}^{2}$

The constants $A$ and $B$ relate purely to the location of the parabola relative to the origin of the graph and, as such, do not describe the curvature. The constant $C$ defines the acuteness of curvature in the parabola such that higher values result in a more acute curve and lower values describe a broader curve and may act as a measure to quantify the approximate degree of curvature in the incisor.

\section{Regression analysis to detect scaling}

Natural log transformed mandible length $(\ln \mathrm{MN})$, mandibular centroid size (ln MNcs), and pronotum-elytra length as an index of body size (ln PEL), were each used as independent variables in a regression analysis on the shape variables derived from the 
GPA using tpsregr v1.38 (Rohlf 2011). Regression analysis using shape variables derived from the GPA yield coefficients representing changes in shape as correlated with the independent variable and the resulting vectors of change are referred to as the "regressions" (Frost et al. 2003). The sums of squares (squared Procrustes chord residual distances) among these residual values measure the residual shape variation seen among the individual configurations once adjusted for the independent variable. The regressions of shape on the independent variables may be expressed as the fraction of shape variance explained by these factors (Frost et al. 2003).

A priori knowledge of the shape differences among the four categories informed me that they were significantly different and discontinuous. As such, a combined analysis of all the groups was deemed inappropriate and groups were analyzed separately for effects of body size, mandibular length, and mandibular centroid size on mandibular shape.

\section{Tests for differences among groups}

Sexual size dimorphism in body size and mandible length within each species was tested using a Welch's t-test for comparing samples of unequal sizes and unequal variances (Welch 1947). Among-group differences in angular measurements for the proximal and distal terebral teeth and mandibular curvature were tested using Tukey's $\operatorname{HSD}(\alpha=0.05)$. All variables were tested for normality with a Shapiro-Wilk test for normal distribution $(\alpha=0.05)$ and transformed as needed prior to statistical testing. Univariate summary statistics, tests for normality, t-tests and Tukey HSD were run using R v2.15.0 (R Development Core Team 2011) through RStudio V0.96.225 (RStudio 2012) 
Sexual shape dimorphism of the mandibles within each species was tested using Hotelling's $\mathrm{T}^{2}$ test of multivariate data $(\alpha=0.05)$ on the partial warps and uniform shape components extracted from the aligned coordinates using tpsRegr v1.38 (Rohlf 2011). The independent variable of sex was arbitrarily assigned the numerical value of -1 for females and 1 for males as suggested in the tpsRegr software documentation (Rohlf 2011).

\section{Results}

\section{Shape analysis of mandibles}

The first two relative warps (RW1 \& RW2) explained nearly $80 \%$ of the observed variation among individuals when the groups were analyzed together (inter-catagorical; Figure 2.8, Table 2.8). By contrast, analyzing the groups separately (intra-catagorical) required a minimum of five RWs to describe $\geq 80 \%$ of the variation (Figure 2.8) meaning that the variables used for this study have little ability to detect trends within the groups but adequately differentiate among the groups. Visually, individuals in the intercategorical analysis form well-defined clusters when plotted on the first two RWs, representing each of the four categories, with RW1 differentiating between the sexes and RW2 separating the species (Figure 2.9). Thin-plate spline visualizations show that travel along RW1 (Figure 2.10) primarily affects the distal region of the mandible, specifically the length and curvature of the apical incisor, with the tooth becoming shorter and more curved in females and longer and less curved in males. Travel along the species differentiating axis, RW2 (Figure 2.11), affects the area of the base of the tooth and mandible, and the angle of the terebral teeth: $O$. audouini have more slender mandibular 
bases, and their terebral teeth, particularly the proximal tooth, are directed more anteriorly relative to $O$. dejeanii, while $O$. dejeanii have more robust mandibular bases with terebral teeth oriented more medially relative to $O$. audouini.

\section{Regression analysis to detect scaling}

None of the natural log-transformed independent variables (ln MN, ln MNcs, and ln PEL) were able to explain more than ca. $7.6 \%$ of the observed shape variation within each group (Table 2.9). Mandible length explained a mean $4.44 \%$ of the observed variation, mandibular centroid size explained a mean $4.56 \%$ of the observed variation and body size was able to explain a mean of $5.54 \%$ of the observed variation. A notable trend is the consistently, and dramatically, lower explanatory values seen in male $O$. audouini with all independent variables relative to the other three groups analyzed.

\section{Tests for sexual dimorphism}

Results of sexual size dimorphism in body size (PEL), mandible length (MN) and mandible centroid size (MNcs) are given in Table 2.10. Both species exhibit femalebiased sexual size dimorphism in body size, with $O$. audouini females $c a .6 \%$ larger than males and $O$. dejeanii females ca. $8 \%$ larger than males. Male $O$. audouini had significantly longer (as measured in millimeters by the linear distance from the articulating hinge to the tip of the apical incisor) and larger mandibles (as measured by centroid size in units of millimeters) than females (14\% larger and 10\% larger, respectively). The larger species, $O$. dejeanii, do not have a statistically significant 
degree of sexual size dimorphism in their mandibles by either length $(t=0.3686$, d.f. $=$ 41.186, $\mathrm{p}$-value $=0.7143)$, or centroid size $(\mathrm{t}=1.3916$, d.f. $=40.488$, $\mathrm{p}$-value $=0.1716)$.

The results of Hotelling's $\mathrm{T}^{2}$ tests confirm that both species show statistically significant sexual shape dimorphism in their mandibles (Table 2.11); however, the magnitude of sexual shape dimorphism is much greater in O. audouini than O. dejeanii, as shown in the relative warp analysis (Figure 2.9).

\section{Angles of terebral teeth and mandibular curvature}

Summary statistics of the angles of the terebral teeth and curve of the incisor are given in Table 2.12 and results of Tukey HSD to determine differences among groups are given in Table 2.13. The angle of the proximal terebral tooth is different between the species and there is sexual dimorphism seen in $O$. dejeanii but not in $O$. audouini. The average angle of proximal tooth for $O$. audouini is $111.25^{\circ}, 5.25^{\circ}$ greater than female $O$. dejeanii and $8.65^{\circ}$ greater than male $O$. dejeanii. The angle of the distal terebral tooth did not show any consistent differences between species or between sexes within the same species with only female $O$. dejeanii and male $O$. audouini being significantly different from each other.

The curvature of the apical incisor, as measured by the C-coefficient of the second-degree polynomial, was significantly sexually dimorphic for both species: females had higher C-coefficient values indicating a narrower curve than males (Table 2.13C). There was no significant difference between females of the two species but males such that $O$. audouini males had the lowest $\mathrm{C}$-coefficient and therefore the straightest incisors. 


\section{Discussion}

Relative warps analysis and multivariate statistical tests identified significant and consistent trends in mandibular shape with respect to sex in the two species of Omus. The dominant axis of shape change (RW1, sex) approximated the unique adaptations these tiger beetles have in relation to their mating behavior, wherein males use their mandibles to grasp the females around the waist. The presence of this behavior throughout the tiger beetle subfamily, suggests consistent trends across species - and even genera - in that sexual shape dimorphism of the mandibles will largely be confined to the length and curvature of the apical incisor and, modestly, the distal border of the anterior terebral tooth, as an accommodation for accepting the female body. A subsequent study looking for possible correlations and covariations among body size, waist morphology, and mandibular morphology, may serve to explain factors related to differing magnitudes of mandibular shape dimorphism among taxa.

In contrast to sex, species level shape variation affected the mandibular base and, in particular, the two terebral teeth of the mandibles, and may be inferred to be related to differences in the prey bases between the two species. We speculate that the relatively anteriorly-oriented terebral teeth of $O$. audouini are adapted for scissor-like shearing and cutting of weakly-sclerotized arthropods (e.g. insect larva, centipedes) and possibly small-to-medium sized non-arthropod invertebrates (e.g. small slugs) while the more robust and relatively medially-oriented terebral teeth of $O$. dejeanii are adapted for crushing and processing the thicker cuticles of more mechanically durable prey such as 
fully-sclerotized adult insects and small-to-medium sized millipedes. Much remains to be known at this time regarding the natural prey base of Omus.

My experience has shown that laboratory-maintained individuals readily accepted lean ground beef and a wide variety of arthropods. Both O. audouini and O. dejeanii consumed live store-bought crickets of nearly equal body length but often had difficulty in capturing them due to the crickets' rapid, saltatory escapes. Terrestrial arthropods such as small- and medium-sized carabid beetles (e.g. Pterostichus sp., Zacotus matthewsii and the non-native Nebria brevicolis) and millipedes (Harpaphe sp.) obtained as by-catch of pitfall trapping were also accepted and noted to have been consumed within pitfall traps. Omus dejeanii were also known to successfully consume $O$. audouini when captured in the same pitfall trap, suggesting the possibility of intraguild predation (Polis et al. 1989) as a potentially significant factor in the ecology of syntopic Omus. Additionally, female $O$. dejeanii cannibalized male $O$. dejeanii on two out of seven observations with pairs kept in $16 \mathrm{oz}$ enclosures for $>24$ hours (See Chapter 5). Cannibalism was likely due to the combination of lack of food and the close quarters of the enclosure. Previously, $O$. dejeanii were speculated to be specialty predators of native millipedes, e.g. Harpaphe haydeniana, on account of frequently observed associations under natural conditions as well as the willingness of $O$. dejeanii to accept and successfully consume Harpaphe (22-40 $\mathrm{mm}$ in length) in the laboratory (LaBonte \& Johnson 1988). We were not able to consistently replicate these results based on four observations of pairing a single $O$. dejeanii (two females and two males) with a single Harpaphe in a $16 \mathrm{oz}$ enclosure for two hours. Only one $O$. dejeanii (a female) penetrated the cuticle and at least partially consumed an adult millipede within the two-hour 
observation period. However, I used larger, mature millipedes $(>50 \mathrm{~mm})$ relative to LaBonte \& Johnson (1988), possibly accounting for the different results. It is entirely possible that the beetles specialize on millipedes of certain sizes.

The partitioning of morphological variation into separate apical incisoral and basilar/terebral regions further suggests the relative flexibility of the role of the mandible in prey capture and processing. While successful mating certainly generates a strong selective pressure, the need to eat is near-constant and starvation may preclude reproduction. From a functional morphology perspective (e.g. Koehl 1996), the localized morphology I speculate to be associated with prey-base (described by RW2 and affecting the basilar-terebral region of the mandible) will have a relatively narrow performance optimum as the opposing teeth need to be precisely complementary to produce the necessary shearing and penetrating forces for prey processing. In contrast, the apical incisor will have a much broader functional optimum and likely used primarily for prey capture - a function indistinguishable from the one used by males in mating - and enjoying much greater morphological variation before an appreciable loss of efficacy.

While none of the $\ln$-transformed independent variables could strongly account for mandibular shape variation ( $<8 \%$ explained), male $O$. audouini had lower explanatory values in all three independent variables, suggesting a modest but consistent sexually selected constraint on allowable morphological variation within the species above and beyond the functional constraints imposed by prey acquisition. This trend was not evident in $O$. dejeanii and may identify a mechanical constraint imposed by prey base.

If $O$. dejeanii are adapted to exploit larger and more durable prey relative to $O$. audouini, their diet would require a greater investment in the mechanical durability of the 
mandibles: not only for processing at the basilar/terebral region (the "nutcracker") but in using the apical incisors to penetrate weak points of prey cuticle and dismembering prey (the "can-opener," see LaBonte \& Johnson 1988 for an account of feeding behaviors in $O$. dejeanii) in addition to initially ensnaring the prey. By contrast, assuming that $O$. audouini are adapted to exploit softer prey, the apical incisor on male $O$. audouini mandibles would have a primary functional application of ensnaring small prey that already fit within the gape of the mandibles with secondary function of penetrating soft structures. Being free from the functional necessities of heavy "can-opener" work, the mandibles of male $O$. audouini were free to elongate and better fit the female without sacrificing the utility of their mandibles as feeding organs. Mandibular deformities of genetic, developmental or traumatic origin have received brief notice in the literature (Pearson \& Vogler 2001, Richardson 2010) but there is no work that cataloges the range of known variations, their causes or their consequences.

The results obtained in the foregoing analyses not only inform us about the possible feeding behaviors and sexually dimorphic accommodations in Omus, but also identify localized functional differences within an organ (the mandible) relating to the multiple functions required of the same that may be applicable to a range of taxa. Morphology has been used to justify niche space inferences for many organisms from the application of Hutchinsonian ratios to identify potential resource partitioning within a community (e.g. Schoener 1974, Lawton \& Strong 1981, Toft 1985), to relative warp analysis on skulls of extinct organisms to determine dietary habits (Figueirido et al. 2009). Recognition of anatomical loci of variability invites further investigation regarding the developmental mechanisms governing the observed variability, 
confirmation of the correlation between morphology and prey base, and the biomechanical consequences and trade-offs of the observed morphologies. 


\section{Chapter 3: Ethogram and Description of Behaviors}

While experimental practices are considered the benchmark standard against which all other science is measured, the value of non-experimental research, work undertaken outside the framework of an experimental system, cannot be understated. This is particularly applicable when considering a poorly studied system. Observational and descriptive studies establish facts from which future questions can be drawn, experiments designed, and hypotheses tested. Behavioral studies often result in anecdotal or non-quantifiable observations that may not directly apply to immediate research questions but still may be useful by suggesting directions for future research. This chapter constitutes a collection of observational and descriptive contributions to the natural history and behavior of tiger beetles (Carabidae: Cicindelinae) in the genus Omus, which constituted the central focus of the work outlined this dissertation.

I preface this chapter by stating that these observations were drawn from contrived, laboratory circumstances, incidental observations, and small sample sizes. It is not known how the experimental enclosures or husbandry practices may alter the behavior of the beetles. The natural forest floor is a highly textured patchwork of many microhabitats and represents a level of complexity irreproducible in a laboratory. Bare soil, leaf mould, and woody debris (both fine and coarse), form a substrate up to several centimeters in depth that is embedded with fallen logs, tree roots and rocks. The floor is further overlain with a variable understory of vegetation and canopy ranging from complete exposure to complete coverage. While the substrate in the beetle enclosures consisted of native materials taken from the collecting sites, the depth of leaf litter was 
limited to a thin surface covering in order to allow for adequate specimen monitoring as part of other studies.

I will initially present an ethogram of typical mating behavior as directly sampled from, and informed by, my personal experiences from reviewing a video library representing over 1,100 hours of video recorded during the summer months of 2011 and 2012. I will then offer a re-interpretation of "female pre-copulatory struggle" as a test of male fitness to a general anti-predator defensive behavior present in both sexes. The chapter will conclude with a report of of sexual cannibalism observed in lab during the 2012 season where a female attacked and consumed a male post-mating on two separate occasions.

\section{Narrative description and ethogram of mating behaviors for two species of Omus A. Introduction: Narrative description and ethogram of mating behaviors for two species of Omus}

Ethograms are essential tools for behavioral analyses. Serving as a catalogue of observable behaviors relevant to a specific context, the information provided in and by an ethogram may then be used to standardize subsequent studies relating to time-budgets, behavioral correlations, and inferences regarding a possible explanation of a behavior, in terms of both ultimate (e.g. evolutionarily) and proximate (e.g. physiological) causes.

Behaviors pre-, syn-, and post-copulation are valuable towards inferring the presence, type, and magnitude of sexual competition, both between and within sexes. Extended or elaborate pre-copulatory behaviors suggest strong female control over the initiation of copulation, such as the female testing the mate for suitability through 
recognition (e.g. species-specific gestures) or accomplishment (e.g. pre-nuptial gifts) cues. The model of differential investment between the sexes relative to parental investment predicts and explains the general indiscretion seen in males regarding mating opportunities and the relative rarity of male-choice mating systems (Bateman 1948, Parker et al. 1972, Trivers 1972). In contrast, syn- and post-copulatory behaviors could suggest either strong male-male competition (as with mate guarding) or, if identifiable as courtship, a degree of control by the female over the latter stages of the reproductive effort (see Table 1.2; Thornhill 1983, Alcock 1994, Eberhard 1985 \& 1996, Birkhead 2010).

A generalized three-stage sequence of mating behaviors has been proposed for tiger beetles (Freitag et al. 1980, Fielding \& Knisley 1995) modeled as (1) initial mount with near-simultaneous intromission, (2) partial or full withdrawal of aedagus while the male remained mounted (presumably to clear any pre-existing seminal products from recent matings out of the spermathecal tract) and (3) a relatively brief reinsertion of aedagus and subsequent dismount of male from female (demonstrated to involve a repositioning of the aedagus within the female genital tract and deposition of seminal products by the current male) (Freitag et al. 1980). However, behavioral variations among and even within species show that this three-stage model cannot be applied to all species (Fielding \& Knisley 1995, Rodriguez 1998). As such, details of mating behaviors from additional species are necessary to define whether a broadly applicable sequential model can even be constructed or whether mating behaviors among tiger beetles are qualitatively different, implying significantly different ecological and populational pressures. 
The specific behaviors and their occurrence during the mating sequence may suggest otherwise cryptic aspects of the reproductive biology of these (and possibly other) beetles. Pre-, syn-, and post-copulatory actions are expected to correlate with stages where one sex has greater or lesser control over the success of the reproductive effort resulting in female-choice or male-choice situations or may be the result of intrasexual competition, as is potentially the case with mate-guarding, or an interplay of one or more of these situations. Varying population densities, operational sex ratios, environmental factors, and anatomical adaptations, all affect the resultant behavior.

As basal members of the cicindeline lineage of carabids, Omus retain many ancestral character states seen in sister carabid taxa, including: forest floor habitat, dark coloration, and non-sexually dimorphic mesepisterna (Freitag 1974, Maddison et al. 1999). As such, the genus Omus stands in a crucial point of transition between ancestral and the more diverse, derived taxa such as Cicindela sensu lato.

\section{B. Materials and methods: Narrative description and ethogram of mating behaviors for} two species of Omus

Three videos of successful Omus audouini matings and three videos of successful O. dejeanii matings recorded over the summer months of 2011 were selected for review. These matings were recorded initially to determine whether there was a difference in mate guarding duration as a function of time of initiation (matings begun in the morning versus matings begun in the evening, see Chapter 4). There was no statistically significant difference in mate guarding duration between the two treatments and the videos were assumed equivalent in terms of representing stereotypical mating behavior 
for Omus audouini and $O$. dejeanii at least in terms of content, if not in frequency of behaviors.

Initial review of the videos revealed that the diversity of stereotypical behaviors occurring during the early stages of mating were similar throughout the entire mating process with the exception of the multiple types of male thrusting (see Fielding $\&$ Knisley 1995 and Rodriguez 1998 for details on interspecific differences in male genital thrusting/genitalic movements). These episodes of male thrusting were brief and occured interspersed between long periods of relative inactivity. The first 10-minutes of mating was reviewed for each video to develop a general catalog (ethogram) of observed behaviors for both females and males of both behavioral states (behaviors continuing for an identifiable period of time, e.g. "the animal is seated") and behavioral events (behaviors that are identifiable as instantaneous or equivalent to instantaneous in time, e.g. "the animal sits") (Altmann 1974).

Videos were then forwarded to one hour after initiation of mating and scanned until at least three distinct episodes of male thrusting (behavioral state) was observed per video. At least one episode was observed before the halfway point between beginning and end of mating, one episode was observed between the halfway point and the end of mating and the third observation period was at an arbitrarily chosen time between the other two observation periods creating a midpoint observation. Male behaviors were described for these episodes to catalog and characterize the types of thrusting (behavioral events). Video was reviewed in both real time and in fastplay (generally x8-16 normal speed) as needed since certain behaviors unnoticeable at normal speeds become evident when viewed accelerated (e.g. male thrusting). 
Oviposition behavior of tiger beetles is highly substrate specific to accommodate the burrowing behavior of the larvae (Willis 1967, Leffler 1979, Knisley 1987, Shivashankar \& Pearson 1994, Hoback et al. 2000, Cornelisse \& Hafernik 2009) and may be expected to influence post-copulatory behavior of the female. Considering the possibility of female control over sperm use, an experimental manipulation was done to evaluate whether female Omus are capable of ejecting speminal products. In 2011, four pairs of $O$. audouini were mated in "minimal environment" containers lined only with wetted paper towel and a few pieces of Douglas-fir cone scale rather than their regular enclosures of native soil and forest debris. The absence of suitable substrate for oviposition was hypothesized to eliciting post-copulatory speminal product ejection - the female would abort the fertilization process rather than invest in eggs that would be destined to fail in an unsuitable environment.

\section{Results: Narrative description and ethogram of mating behaviors for two species of Omus}

An ethogram of behavioral states and events observed for mating Omus is provided in Table 3.1. Stereotypic overt mating behaviors were readily discernible, and all behaviors listed in the ethogram, both states and events, were identified in two videos, the third video serving to confirm the recurrent nature of the behaviors. Four behavioral states were identified and indicated with two-letter codes while seven distinct and discrete behavioral events were defined and represented by three-letter codes. One of the behavioral states, "both still" (BS), may actually represent two different cryptic physiological states: one of a short term "pause" (duration $<5$ minutes) and the other 
being a longer rest period (duration $>30$ minutes) possibly involving metabolic depression or a "sleep" state. Unfortunately video alone did not provide evidence to definitively differentiate between these states; they are thus reported as a single category.

\section{Description of general mount posture}

No pre-contact/pre-copulatory courtship behaviors were observed or obviously discernable. Pre-copulatory struggle by the female was seen as a frequent response to male contact in both species. This struggle only rarely prevented males from mounting the female. Upon contact with the female, the male attempted to reach a mounted position, orienting himself as needed using his forelegs and mandibles. The male would then grasp the female around the waist with his own mandibles and attempt to insert his intromittent organ in the female. Occasionally the female would struggle at the attempt of intromission, the struggle apparently interfering with the male's attempt to insert his aedagus. When the female responded with a struggle, the male would begin to vigorously stroke and drum the head and antennae of the female with his own antennae as well as, less obviously, use his own hind legs to stroke the hind legs of the female. A few moments of stroking by the male would result in an eventual cessation of female struggle and subsequent intromission.

\section{Syn-copulatory behaviors}

Once mounted and inserted, the male's legs were held outstretched or allowed to drag (tarsal pads of first legs only used for initial mounting effort - once successfully mounted, forelegs are generally held outstretched). The male's position is maintained by 
at least three points of anterior contact formed by the left and right mandibles against the lateral areas of the female's mesepisternum and the male's labrum contacting the dorsal surface of the female's waist. Additionally, the inserted genitalia provided another point of contact and mechanical stabilization for maintaining male position. In contrast to the mating behavior reported for other tiger beetles (Cicindela marutha: Kraus \& Lederhouse 1983, Cicindela dorsalis and C. puritana: Fielding \& Knisley 1995, Pseudoxychila bipustulata and P. tarsalis: Rodriguez 1998), Omus were observed to maintain genital contact with at least partial insertion throughout the duration of the mount.

The mounted female is mobile, successfully ambulating around the enclosure despite the encumbrance of the male, using her antennae and mandibles to contact and explore the environment. While a mounted female is likely be handicapped in her effort to hunt, mounted females were observed to ingest items such as hydration gel and chew on substrate while mounted. Females of other tiger beetle species have been reported to successfully hunt, eat, and drink while mounted (Willis 1967), and there is no reason to think the same is not the case of Omus. The male does not directly assist in female movement and, at times, acts as a handicap. If the male's legs get caught on substrate, the male may kick away to dislodge himself or may not, relying of the female to drag him away from the encumbrance. Otherwise, the male keeps his legs held aloft or allowed to passively drag along the substrate.

While mounted, the male's antennae are held laterally or slightly forward in a relaxed state (as opposed to actively addressing the environment) unless being used to stroke or drum the female. Syn-copulatory courtship behaviors of the male, predominately stroking and drumming the head and antennae of the female with his own 
antennae, were not unlike those reported in Rodriguez (1998) and Fielding \& Knisley (1995). Intromission behaviors (thrusting) were stereotypic and distinctive between the two species with $O$. audouini males engaging in long bouts of slow thrusts (each insertion/withdrawal cycle taking several seconds to complete). By contrast, $O$. dejeanii males generally engaged in short bursts of rapid thrusts, each cycle taking a second or so to complete.

\section{Copulatory thrusts}

For O. audouini:

Three different types of thrusts were observed in intromission of $O$. audouini and detailed as behavioral events (Table 3.1). Thrusts usually occurred in bouts of a single type performed one after another with little to no pause in between. An episode of male thrusting for $O$. audouini would typically last an average of 72.3 minutes $(\mathrm{SD}=30.5$ minutes, $\mathrm{n}=4)$ with a mean rate of 1.5 thrusts/min $(\mathrm{SD}$ $=0.3 \mathrm{t} / \mathrm{m})($ Table $3.2 \mathrm{~A})$.

For O. dejeanii:

Four different types of thrusts were observed for intromission in $O$. dejeanii and detailed as behavioral events (Table 3.1). In contrast to O. audouini, the copulatory behavior of $O$. dejeanii was more complex and not conducive to simple thrust counts. An episode of male thrusting for $O$. dejeanii would typically last an average of 110.5 minutes $(\mathrm{SD}=38.6 \mathrm{~min}, \mathrm{n}=4$; Table $3.2 \mathrm{~B})$ with multiple short pauses and differences in thrust types and rates (Table 3.1). 


\section{Female syn-copulatory struggle and discontinuation of mating:}

Females struggled occasionally (syn-copulatory struggle) while the male was mounted; however, this never resulted in the male being dismounted ( $\mathrm{n}>40$ mating observations over two years). At times, the male would begin thrusting and the female would engage in a syn-copulatory struggle. The male would then rapidly stroke the head and antennae of the female with his own antennae, whereupon the female would generally cease struggling. This male behavior is similar to that seen during initial intromission efforts, which also results in female struggle. This cycle of beginning thrusting-copulatory struggle-antenna stroking would occasionally be repeated several times before a male would engage in thrusting without interruption, leading me to hypothesize that the antennal stroking is an essential form of syn-copulatory courtship and necessary to stimulate the female into allowing thrusts from a mounted male.

Discontinuation of intromission and subsequent dismount was preceded by a female struggle, or may occur without any overt behaviors from the female: the male simply discontinuing contact. Serial mounting, where the male would remount the same female, was a frequent event and could occur anywhere within a few seconds or up to an hour or more after dismount, suggesting that there is no physiological refractory period to the mating behavior. Whether there is an ejaculatory refractory period remains unknown.

\section{Post-copulatory behavior: spermatophore ejection}

All four pairs successfully mated within the minimal environment of wet paper towel rather than native soil. After ca. 19 hours, the experiment was discontinued, and 
the subjects separated and returned to their regular enclosures. The minimal environment enclosures were then inspected for evidence of spermatophores. Two of the enclosures were found to contain small, cream-colored, gelatinous products similar to spermatophores described for Pseudxyochila sp. (Rodriguez 1998) suggesting that female O. audouini-and likely other Omus species — have the ability to eject seminal products post-copulation. Simple inspection of the substance under a dissection microscope was unable to confirm the presence of actively motile sperm or cellular structure. It is unknown whether the substance was a copulatory plug, a sperm-containing structure where the sperm had either departed or were unresolvable with the visualization methods used or a non-gametic seminal product related to either the sustaining of the sperm or, possibly, nutrient supplement for the female (a post-copulatory nuptial gift). In the case of a post-copulatory nuptial gift, it is unknown whether the standard practice would have the female eject and ingest the product or is directly absorbed into the body. Furthermore, the seminal product may contain compounds that either inhibits future mating beyond simply blocking the genital tract (an anti-aphrodesiac, see Birkhead 1995) or hormonally assists fertilization, both situations committing the female to commit to the current brood despite unsuitable conditions (i.e. inappropriate substrate).

\section{Same-sex interactions in Omus audouini}

Male-male interactions: In the absence of a female, males would regularly attempt to mount one another and maintain position for a few seconds to, in a single pair of O. audouini, 1.6 hours. Occasionally, the mounted male would have his aedagus extended and buried in the substrate, the contact possibly providing the 
stimulus to remain mounted. Same-sex mating efforts have been observed in many animal taxa and can be attributed to reasons of mate misidentification, endogenous reward (i.e. sexual pleasure), social bonding (Bagemihl 1999) and social dominance (Chiroro et al. 2004, Chapleau et al. 2010). Additionally, males of the animal kingdom are notoriously promiscuous and, if not implicated in parental care, generally benefit more from taking advantage of as many mating opportunities as possible rather than insuring any single mating event is successful (Bateman 1948, Parker et al. 1972, Trivers 1972). Social structure and enduring bonds have not been observed within the subfamily Cicindelinae-all members acting as non-social predators without parental care. Additionally, fullsequence mating-type behaviors performed to completion in the absence of a partner or partner-surrogate (i.e. sexual autostimulation or "masturbatory" behaviors) have been neither observed nor described for tiger beetles. In light of the lack of social structure and absence of what could be termed masturbatory behaviors within the taxa, the single observation of enduring male-male mating effort is most likely attributable to mate-misrecognition.

In the presence of a female, males also would mount one another; however this behavior would usually be rapidly discontinued, and generally occurred when a male encountered another male while in pursuit of a female. This suggests the male had been primed with a possible fixed action pattern related to mating, rather than a general response to conspecifics. Additionally, the willingness to commit to an extended mating attempt with another male invites questions regarding mechanisms of mate recognition. While the role of cuticular 
hydrocarbons as contact pheromones in insects is well known in general (e.g. Singer 1998), there is little literature and little evidence relating to the reliance on chemical cues for recognition or communication among tiger beetles. Alternative hypotheses involve tactile cues such as the shape of the female waist as contributing to mate recognition (Freitag 1974, Kritsky \& Simon 1995, Kritsky \& Reidel 1996). However the ancestral state of the mesepisterna in Omus defies using it as the most obvious species- and mate-recognition cue. At present, I do not know of any studies related to "indiscriminate" mating attempts for male tiger beetles that specifically address how often a male of one species may attempt to mate with a "non-reproductive" option such as a same sex conspecific or a heterospecific. Such research would be valuable towards detemining whether recognition cues for males are predominately morphological, chemical or behavioral.

Female-female interactions: While aggression was noted between females, mating-style mounting attempts were not observed for any female-female pairings. Nor did I witness any behaviors suggesting a committed attempt of intrasexual cannibalism between females. Female-female interactions were either "neutral"—where the beetle didn't react to the female that was noticeably different from any other part of the environment; "investigative"—where one female would pause and tap the other with antennae or mandibles but not engage in any subsequent behaviors or "aggressive" — the female either gaping her mandibles or 
actively biting the other female, with one or both beetles subsequently retreating from the contact.

\section{Discussion}

The three-stage mating sequence of initial insertion-withdrawal-final insertion and dismount does not appear applicable to these species of Omus. Rather, Omus mating is better modeled as a four-stage behavior of: (1) Initial mounting, (2) Courtship with intromission, (3) A variable number of episodes of syn-copulatory courtship/thrusting/male quiescence and (4) Final withdrawal coupled with an immediate dismount.

Research on water striders (Hemiptera: Gerridae), which also engage in malemounted mated for extended periods (7-145 min depending on species, Fairbairn 1990), shows both members of the pair suffer handicaps in mounted reproductive postures even if the female is not near her load-bearing limit (Fairbairn 1993, Rowe 1994). The apparent freedom of movement for the mounted female therefore may be relative and actually represent a handicapped state in terms of agility and speed. There are no studies comparing the physiological costs or foraging handicaps of mounted versus non-mounted female tiger beetles.

I once attempted to manually separate a pair of $O$. audouini (not included in the dataset) within one minute after mounting. With effort, I was able to pry the male mandibles away from the female. However, the male did not withdraw his aedagus despite attempts to gently pull the beetles apart. Further force was not used out of fear of injuring the subjects. On release, the male immediately replaced his mandibles around 
the female's waist and mating resumed without note. The reluctance of the male to withdraw once in place and the apparent ineffectiveness of the female pre-copulatory struggle (and even syn-copulatory struggle) suggests that the actual mounting of the female by the male and time of eventual dismount is likely male controlled. However this does not preclude the possibility of female controlled stages of the reproductive effort occurring after initial mounting, such as genitalic movements, sperm placement by the male within the female genital tract, and the eventual retention or ejection of the spermatophore by the female. Spermatophore ejection, seen here in Omus audouini, has been observed and reported in Pseudoxychelia (Rodriguez 1998) as well as in other carabid beetles (Takami 2007). Similar behvior has not been explicitly noted for many tiger beetle species and may be an ancestral character retained in basal lineages but lost in derived taxa.

Mating behaviors in Omus superficially appear to be predominately male choice however this does not discount the presence of simultaneous female choice mechanisms occurring during or after copulation. It appears that males exercise control over the initial mounting and duration of contact as evidenced by the difficulty in sepeerating a mating couple, either by female struggle or by an external agent. In turn, the lengthy mating duration as addressed in Chapter 4 allows hypothesizing about the presence of female choice via syn-copulatory courtship. The repeated initial male thrust-female struggle-male antennal stroking-female tolerance-male thrusting sequence strongly suggests female control over aspects of the insemination process. The violent struggle can be predicted to prevent the male from effectively accessing regions of the female genital tract, which, in turn, determines the placement of the sperm. Another 
consideration is that the struggling of the female precludes insemination by the male. It may, therefore, be hypothesized that the extended mating duration of Omus is a product of selection for courtship in the face of strong female control over the fate of the sperm.

\section{Pre-copulatory struggle, a proposed reinterpetation}

The three vital evolutionary pressures of food acquisition, predator defense, and reproduction, intersect in the mating behaviors of tiger beetles (Carabidae: Cicindelinae). The generalities of tiger beetle reproductive behavior appear consistent among the tiger beetle species so far examined, although few species have been studied in detail in this regard. In brief, males pursue and attempt to mount a female without overt pre-contact courtship. The male grasps the female around the waist with his mandibles, to which the female often responds by engaging in a pre-copulatory struggle ("PCS"). If the male maintains position, he then everts and inserts his aedagus and the pair engages in coitus, with the males generally remaining mounted post-coital (or between intromission events) as a form of contact mate guarding (Kraus \& Lederhouse 1983, Shivashankar \& Pearson 1994, Fielding \& Knisley 1995, Rodriguez 1998).

Through the course of mating, male beetles engage in hunting and seizing behavior not unlike, if not indistinguishable from, prey seeking and capture, while females are subjected to spontaneous episodes of unannounced contact and attempted seizure. The phenomenon of PCS has been characterized as a female choice mechanism, either a test of mate identity as in the case of species bearing coupling sulci or as a test of male "quality" such that "unfit" males will be unseated and copulation prevented (Pearson \& Vogler 2001). Both hypotheses presuppose that, by the moment of contact, 
the male has communicated his intent to mate and the female has differentiated the male from any other type of environmental interaction, such as contact with a potential predator or similar threat. The purpose of this study was to assess whether PCS could be interpreted in the context of anti-predatory defense behaviors.

During the course of observing same-sex conspecific behaviors, males of both species would attempt to mount conspecific males. Heterospecific pairings were not attempted because of the potential of predation on $O$. audounini by the larger $O$. dejeanii, a frequent event in pitfall trapping of live specimens.

While the struggle behavior is assumed to be present in females due to their highly invested reproductive role, the same assumption could not be made of males since they lack the potential for egg production and its associated costs (Bateman 1948, Trivers 1972). If PCS was primarily a test of mate quality, male-male struggle should not be expected. If, in contrast, the observed pre-copulatory struggle is a general anti-predator response, struggle events - or behaviors indistinguishable from PCS - readily observable in both sexes upon initial contact and mount.

Out of ten male-male pairings of $O$. audouini, all had at least one mounting attempt within five minutes of initial introduction. Six initial mounting attempts (60\%) resulted in a violent response indistinguishable from what has been described for female pre-copulatory struggle. In comparison, ten female-male pairings arranged under similar conditions resulted in seven violent responses to initial mounting (70\%).

These preliminary observationssuggest that struggle behavior may be a general response to being seized independent of the sex of the beetle being seized. Male $O$. audouini appeared to habituate more rapidly to repeated contact (mounting efforts) and 
responded less frequently with a struggle. In contrast, females continued to respond with violent behavior as a response to contact from the male.

A predator may be prey for another predator, and even apex predators are susceptible to cannibalism (Fox 1975) and intraguild predation (Polis et al 1989). Avoiding predation is arguably more immediately decisive for the fitness of an animal than finding food or mates: it is easier to recover from hunger than from dismemberment and, thus, better to face temporary uncertainty of reproduction than certainty of sudden, irreversible death. Antipredator mechanisms range from passive (e.g., armor) to active (e.g., retaliatory attack), and these features may function by themselves or in combination to benefit the animal. Many antipredator mechanisms may be considered multi-use and contribute to a broader suite of applications including defense, hunting, intrasexual competition, and environmental manipulation (for example, scorpion claws, male primate canine teeth, and lagomorph hindclaws, are all very effective at discouraging unwanted contact while serving alternate functions).

Rather than a novel development for testing suitor quality, pre-copulatory struggle in females of the genus Omus may be interpreted as an anti-predator response present in both sexes but further extended to serve a secondary purpose of testing mate quality. Conceievably, in the absence of pre-mounting courtship signals, the female is erring on the side of caution pending positive identification of the intent of the contact through courtship signals.

Tiger beetles are endowed with a suite of defenses to survive an attack (Pearson \& Vogler 2001, Pearson 1990; also Table 3.3) but whether any given character was initially adapted towards defense rather than secondarily selected for defense remains to be seen. 


\section{Post-copulatory intersexual cannibalism in Omus dejeanii}

\section{A. Introduction: Post-copulatory intersexual cannibalism in Omus dejeanii}

Cannibalism, defined here as intraspecific predation, has been documented across many taxa and appears to be at least a somewhat regular occurrence within natural animal populations (Fox 1975, Elgar \& Crespi 1992). Often noted among generalist omnivores as a result of excessive food deprivation or increased population density (Fox 1975, Elgar \& Crespi 1992), there are potential selective advantages to the genetic maintenance of the willingness to consume one's own kind (Fox 1975, Buskirk et al. 1984).

Cannibalism is known to occur within, as well as among, different life stages, including adults consuming eggs, juveniles, or other adults, juveniles consuming eggs or other juveniles (Elgar \& Crespi 1992), and even, rarely, filial cannibalism wherein adults consume their own young (Bartlett 1987, Stevens 1992) and matriphagy/patriphagy where young consume their own parents (Toyama 1999, Kim et al. 2000, Suzuki et al. 2005, Tizo-Pedroso \& Del-Claro 2005). In known cases of sexual cannibalism, the female may consume the male pre-, syn-, or post-insemination/copulation. Preinsemination sexual cannibalism is clearly not associated with male reproductive success and instead better viewed as either a foraging option by the female or a failure of courtship by the male (resulting in mistaken identity by the female or a rather severe example of female choice mate rejection) (Elgar 1992). Syn- and post-copulatory sexual cannibalism may be argued as possibly advantageous for the male, contributing to his own fitness by contributing to the fertility of the female through nutrient provisioning 
pursuant to the parental investment hypothesis (Thornhill 1976, Buskirk et al. 1984, Arnqvist \& Henriksson 1997).

During a series of behavioral observations examining the effects of food deprivation and operational sex ratio on the mating duration of two species of night stalking tiger beetles (genus Omus), two instances of successful post-copulatory sexual cannibalism by a single female were observed and recorded on video. These events resulted in the fatality, dismemberment, and consumption of the male. Considering the value of well-detailed accounts, even anecdotal and unplanned behaviors, towards identifying directions of research and formulating future studies, these two events are detailed herein.

B. Materials \& methods: Post-copulatory intersexual cannibalism in Omus dejeanii

Several pairs of wild-caught Omus audouini and O. dejeanii were recorded for a study of mating behavior during the 2012 season. Food availability and operational sex ratio were manipulated to examine their effects on mating duration. Upon conclusion of the observation periods, I noted that a female used in two separate experiments had cannibalized the male on both occasions. Videos were reviewed and the events related to these episodes were documented. Direct body length could not be determined for all participants because the males had been dismembered and mutilated. Instead, the combined lengths of the pronotum and elytra measured separately are given as a body size index (BSI) for comparison. All BSI are given for participants as well as the proportion of cannibalizing female BSI to cannibalized male BSI. 
C. Results \& discussion: Post-copulatory intersexual cannibalism in Omus dejeanii

\section{Narrative/description of events}

Out of eight recorded experiments involving mating O. dejeanii during the 2012 season, two matings resulted in male fatalities and cannibalism by a single female. A third mating involving a different female $O$. dejeanii showed behaviors suggestive of attempts at cannibalism of the male; however the experiment was concluded prior to any male fatality. Only a single pair of $O$. audouini showed any females briefly demonstrating behaviors suggesting a possible attempt to cannibalize the male, but these were largely indistinguishable from behaviors seen in simple, defensive aggression. All females had been food deprived for a minimum of 40 hours before attacking the male and, due to the objectives of the experiment (comparing effects of food deprivation and operational sex ratio on mate guarding duration), at times the female had been deprived of food for as long as 84 hours ( 3.5 days) prior to attempting to cannibalize the male. Similar behaviors by the males directed toward the females were not observed. The behaviors of the two events resulting in male fatality are presented in detail since one occurrence was under specifically manipulated conditions of food deprivation while the other occurred under a female-biased operational sex ratio (two females and a single male) and the male was specifically targeted by one female while the other female was not similarly attacked. Screen shots of the progression of events for the food deprivation experiment are provided in Figure 3.1. Similar pictorial treatment for the operational sex ratio experiment was regarded as redundant and not included.

\section{Food deprivation experiment observation}


Date of experiment: 09 July 2012

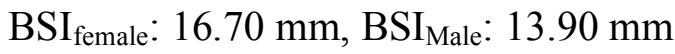

BSI Proportion: Cannibalizing female/Cannibalized male $=1.20$

Two beetles, one male and one female, were contained within an enclosure to test the effects of female food deprivation on mating duration. The female had been allowed to feed ad libitum for one hour, then food deprived for 24 hours, while the male had been allowed to feed ad libitum one hour prior to introduction. The male mounted the female after 21 seconds of introduction to the enclosure and maintained amplexus continuously for over 35 hours. The pair ended amplexus at 35:53:59 upon dismount by the male without observable struggle by the female. Several brief $(<10$ seconds) bouts of reciprocal pursuit occurred where the two beetles contacted each other and the male attempted to mount the female while the female attempted to seize the male, resulting in a chase around the circular enclosure. Otherwise, the subjects remained motionless, ambulated around the enclosure, or chewed on substrate or water gel. At 36:17:03 the female seized the male around the waist and maintained contact either in that position or with the male's remains until ca 41:47:54 (5.5 hours), at which point the male had been fully dismembered and consumed. The female spent much of the pre-dismemberment time slowly chewing at the waist-joint of the male. There was some evidence of a wet fluid - possibly digestive fluids - being secreted from the oral region of the female throughout the event. Alternatively, the observed fluid could be the male's own hemolymph on account of the traumatic injury being inflicted. Pre-dismemberment, the male ambulated around enclosure and occasionally struggled. At ca. 37 hours, male was seen still struggling but was more carried by the female in her mandibles than self- 
ambulating with the female. At 37:34:20, the female was seen to press the abdomen of the male against the substrate and at 37:34:55, male became sharply bent at the waist joint, indicating possible partial disarticulation. Full disarticulation was noted at 37:47:27, when the male abdomen became visibly separated from the remainder of body. The female consumed the contents of the head and thorax of the male first, then the contents of the abdomen, ultimately ingesting most of the soft tissues of the male. The female abandoned the male remains at 41:49:12. Upon examination, the male cuticle was noted to be largely broken into pieces with few external structures left intact (Figure 3.1).

\section{Female-biased operational sex ratio experiment observation}

Date of experiment: 14 July 2012

BSI $_{\text {Female A }}: 15.75 \mathrm{~mm}, \mathrm{BSI}_{\mathrm{Female} \mathrm{B}}: 16.70 \mathrm{~mm}, \mathrm{BSI}_{\text {Male }}: 14.75 \mathrm{~mm}$

BSI proportions:

Cannibalizing female (female B) / Cannibalized male $=1.13$

Non-cannibalizing female (female A) / Cannibalized male $=1.07$

Cannibalizing female (female B) / Female $\mathrm{A}=1.06$

Three beetles, two females and one male, were contained in a single enclosure in order to test the effects of female-biased operational sex ratio on mating duration. All subjects had been allowed to feed ad libitum for one hour prior to introduction. The male mounted female B at 00:17:02 and dismounted at 00:18:55. The male remounted female $\mathrm{B}$ at 00:22:05 and persisted in continuous amplexus for ca. 28 hours. The male dismounted female B at 28:21:08. After 14:32:43 (timestamp 42:53:51), Female B seized the male. The male violently struggled in response to being held but was unable to 
remove the female. Female B maintained this contact, walking around while carrying the male. The elytra of the male became detached at 43:18:02. At 43:26:26 female B appeared to be aggressively masticating the male at the point of the waist joint between bouts of ambulating around the enclosure with the male held in her mandibles. The male's thorax became obviously separated from the abdomen at the point of the waist joint at 43:35:20 at which point female B began consuming the male. Female A was not observed to participate in either the killing of the male or in consuming his remains, possibly because female B did not abandon any part of the male remains until they had already been stripped of edible tissues. Neither was female A seen to try and steal or fight female B for access to the male's remains. Female B abandoned the male remains at 46:38:33 (total duration: 3:44:42). Upon examination, the male cuticle was broken into several pieces and widely dispersed throughout the enclosure.

In the time between the end of mating and the time that female B secured a persistent hold on the male, female B several times unsuccessfully attempted to grasp the male. Female A was contacted regularly by both the male and female B, however female B did not appear to attempt to seize and consume female A. Instead, the females either did not acknowledge each other (a neutral response such as walking over one another), or briefly explored each other with their antennae and mandibles before disengaging or biting at the other (possibly in defensive/warning gestures) but not pursuing or engaging in conspicuously predatory attempts.

\section{Discussion \& Conclusion}


Sexual cannibalism is expected when (1) the male has a relatively low probability of remating and (2) the nutrient contribution provided by the cannibalistic act significantly contributes to increased quality or number of offspring (Buskirk et al. 1984, Arnqvist \& Henriksson 1997). In many taxa demonstrating sexual cannibalism, the female consumes the male before insemination therefore invalidating the assumption of any reproductive advantage to the male (Arnqvist \& Henriksson 1997, Elgar 1992).

Cannibalism carries several costs, not only to the individual but also to the population if established as a regular behavior. Increasingly generalized foraging practices would involve a lowered standard regarding what is recognizable or acceptable as prey — a choice that may venture into unhealthy decisions such as noxious or pathogenic items or dangerous prey, essentially reversing previously selected cues relating to palatability (Elgar \& Crespi 1992). Cannibalism also carries a possible loss of personal fitness through a relaxation of kin-recognition and subsequent killing relatives, effectively decreasing the individual's genetic representation within the population (Elgar \& Crespi 1992). However, considering the presence of these costs and the persistence of cannibalism, it follows that there must be concurrent benefits of equal or greater magnitude — at least under certain conditions — that enable such behavior to persist (Elgar \& Crespi 1992).

For the events described above, the tiger beetles clearly engaged in postcopulatory sexual cannibalism, allowing for the potential that the behavior in this group of organisms may in fact be a form of parental investment, mainly directed by the female in light of the supposed lack of male complicity: he was actively pursued and captured rather than quietly acquiescing to consumption. Considering that these were the only two 
instances of sexual cannibalism witnessed among Omus in 53 recorded matings ( $\mathrm{n}=32$ pairs of Omus audouini, $\mathrm{n}=21$ pairs of $O$. dejeanii) over two years (2011-2012) and involved the same female, I suspect the frequency of sexual cannibalism under natural conditions is low.

A more likely explanation is that the observed behavior was an artifact of the laboratory circumstances of food deprivation and limited space. Tiger beetles, including Omus, are known to be promiscuous (several mating events in their lifetime) and to carry out multiple mating (several partners), which violates one of the two predictors of selection for sexual cannibalism as parental investment. In addition, cannibalism was not attempted until quite some time after the male had dismounted (23 minutes for the food deprivation experiment, over 14.5 hours for the operational sex ratio experiment). Under natural circumstances, the male and female would likely have been afforded enough space to distribute themselves within that time frame and avoid further contact. The experimental framework does not, however, preclude the possibility of females encountering other males and attempting to consume them as prey, nor does it address the preference for the female to attack the male while leaving the other female unmolested as seen in the operational sex ratio experiment. 


\section{Chapter 4: Mating: Sexual Size Dimorphism and Behavior}

\section{Mating positions and sexual size dimorphism in carabid beetles and Omus \\ A. Introduction}

The posture assumed between partners during coitus may be assumed to have consequences, both advantageous and disadvantageous. Depending on specific physical constraints, such as overall mass, shape of anatomy, and flexibility of joints, certain mating postures may not be possible for an organism (e.g. it is unlikely elephants would anatomically be able to engage in ventral-ventral coitus without traumatic injury), while other species may have greater freedom in coital positioning and enjoy more than one positional option (e.g. primates).

In addition to intra- and inter-sexual competition, where certain postures are likely to be more conducive to resisting displacement by competing mates or resisting females, coital position likely is also heavily influenced by the presence of pronounced sexual size dimorphism. A large dissimilarity in size between sexes may burden or benefit the mating pair depending on their relative positions. In extreme cases, one mate may risk crushing the partner during contact due to exceeding the load bearing limits (e.g. southern elephant seals, Mirounga leonine, where males may be seven times the mass of the females and occasionally injure their mates, Fairbairn 2007). Alternatively, an opposite situation may occur where the less massive partner allows easier, less stressful mating, as in the case of female-biased sexual size dimorphism coupled with male-superior mountings.

Male-superior ventro-dorsal mating (MSVD) posture, where the ventral surface of the male contacts the dorsal surface of the female while both individuals are facing in the 
same direction, is, if not the rule, very common among terrestrial animals with internal insemination.

All known tiger beetles engage in MSVD, where the male rests on the female, causing the female to bear most or, even, all of the weight of the male. Prior studies on water striders, which exhibit a similar mating posture and duration as tiger beetles (2-24 hours, Wilcox 1984), have identified significant disadvantages to entirely female supported MSVD — particularly in situations with extended amplexus or contact mate guarding - including increased physiological stress on the female and greater risk of predation to both partners (Fairbairn 1993, Rowe 1994; but see Wilcox 1984 where foraging by the female was enhanced by mate guarding).

Prior studies have established that carabid beetles generally show a modest degree of female-biased sexual size dimorphism that is approximately isometric with $\beta=0.97$, i.e. the magnitude of SSD is constant and independent of body size - females of both large and small species are ca 7-8\% larger than males, (Blanckenhorn et al. 2007b).

Among tiger beetles, there is variation in body size and mate guarding duration as well as differences in habitat type, ranging from forests (for the most basal genera, e.g., Picnochile, Omus), to the semi-arid and arid habitats of the more derived genera (e.g., Cicindela sensu lato). It may be hypothesized that, due to mass increasing as a cubic function of linear dimension, mounted mate guarding among larger-sized insect species (which also have longer mate guarding durations, i.e., Omus) would constitute a proportionally greater burden for the female relative to smaller species and select for greater female-biased SSD to decrease stress on the female. I hypothesized that tiger beetls of the genus Omus would show a greater magnitude of SSD than seen in tiger 
beetles of the genus Cicindela. Tiger beetles of the Cicindela complex are medium- to small- bodied, endemic to arid and semi-arid habitats and engage in mating behaviors generally for less than one hour (Table 1.3). In contrast, Omus are relatively large-bodied among tiger beetles, endemic to sheltered, forested habitats of the west coast of North America and engage in extended durations of mating at least an order of magnitude longer than reported for any of the genus - all features that would, presumably, generate selective pressure for greater proportional differences in the sexes (increasing femalebiased SSD).

\section{B. Materials and methods}

To test whether tiger beetles maintain or deviate from SSD isometry, I directly measured samples from two species of field-captured night stalking tiger beetles (Omus audouini and O. dejeanii; all specimens captured with pitfall traps at Powell Butte Nature Park, Portland, Oregon) and collected body length data of six species of North American Cicindela sensu stricto from published sources.

Body length was measured on 70 O. audouini females, 70 O. audouini males, 43 O. dejeanii females and $50 O$. dejeanii males. Body length was measured to the nearest $0.5 \mathrm{~mm}$ with dial calipers, and defined as the linear distance from the posterior tip of the right elytron to the most anterior medial prominence of the labrum. Equivalent body length data for six other tiger beetle species were drawn from Kritsky \& Simon (1995) and Kraus \& Lederhouse (1983).

These eight species of tiger beetles represent a gradient of body sizes from under $9 \mathrm{~mm}$ to over $20 \mathrm{~mm}$ in length, mating durations from less than 5 minutes to over 16 
hours, and categorically different habitats (river banks and salt flats for Cicindela spp; west coast forest floors for Omus spp) (Table 4.1).

The degree of SSD was quantified for each species as per the size dimorphism index (SDI) of Lovich \& Gibbons (1992), where SDI $=[($ female body size $) /($ male body size) - 1] for female biased SSD systems. The relationship of body size to SSD was found by reduced major axis regression of $\ln$ (male body length) onto $\ln$ (female body length) to determine the scaling factor, $\beta$. Statistical analysis was performed using $\mathrm{R}$ v2.15.2 (R Core Development Team 2011).

\section{Results}

Body length measurements for Omus are given in Table 4.1A. Combined body length data for all eight species and SDI is given in Table 4.1B. The tiger beetles examined here have a mean sexual size dimorphism of $8.7 \%$ ( \pm SD $2.2 \%$, range: $5.7 \%-$ $12.2 \%$ ), i.e., female tiger beetles are generally $8.7 \%$ larger than their males. Plotting and regressing the data reveals a nearly isometric relationship (Figure 4.1). The reduced major axis regression line equation yielded a scaling factor of $\beta=0.98(2.5-97.5 \% \mathrm{CI}$ : $0.89-1.07)$.

\section{Discussion}

Mating postures among tiger beetles are confined to the single posture, therefore imposing a consistent and predictable burden at each mating. Unexpectedly, we do not see a change in the magnitude of SSD across tiger beetle body sizes, mating duration or habitat type. Selection for body size has been modeled as an equilibrium between 
juvenile viability selection encouraging shorter development times to sexual maturity (resulting in smaller body sizes), and either fecundity selection for females or sexual selection for males, both encouraging larger body sizes maximizing offspring quality or direct intrasexual competitive ability, respectively (Blanckenhorn 2000). While it may be predicted that optimal body size is different for each sex, the degree of sexual size dimorphism is dependent on the genetic correlation between the sexes, constraining the degree of differences (Reeve \& Fairbairn 1996, Blanckenhorn 2000).

The isometry of sexual size dimorphism across a broad range of body sizes and mating durations for tiger beetles suggests powerful genetic constraints within Carabidae that restrict deviation from anything more than modest size differences between the sexes. Other insect families are known to show non-isometric scaling in the degree of sexual size dimorphism, both in accordance with, and in opposition to, Rensche's rule (Teder \& Tammaru 2005, Blankenhorn et al. 2007b). However, SSD patterns identified for insects are inconsistent and taxon specific, suggesting either an uneven distribution of selective advantages, or uneven distribution in genetic correlation between sex and body size despite selective advantages such as female load bearing ability (Blankenhorn et al. 2007a). A survey of mating positions among insects would constitute an invaluable framework in the path to uncovering the costs and benefits of any given posture.

\section{Mate guarding in Omus: effects of species, food, operational sex ratio \& time on mating duration}

Mate guarding is described as a strategy towards assuring paternity (Parker 1970 \& 1974, Alcock 1994) and has been documented and explored in numerous animal 
species. While the male gains an increased certainty of paternity, significant costs and consequences exist for both partners. Not only is there a time investment that detracts from seeking other, potentially more successful mates (Parker 1974), the mate guarding commitment may also increase physiological stress due to exposure, energy expenditure due to competition with other males and following the female, and risks from predation, since mate guarding handicaps defensive behaviors such as hiding (Cothran 2004, Fairbairn 1993, Rowe 1994, Saeki et al. 2005, Watson et al. 1998).

Tiger beetles (Carabidae: Cicindelinae) are specially adapted to male-superior dorso-ventral mating posture with the male maintaining position by grasping the female around the waist with his mandibles (Pratt 1939, Freitag 1974). The male mandibles contact the female mespisterna, a bilateral pair of plates formed from the exoskeleton. The mesepisterna form the ventro-lateral aspect of the tiger beetle "waist" - the constriction between the thoracic and abdominal segments. Not only do females of several genera exhibit specialized sulci on their mesepisterna to preferentially accept and match male mandibles (Freitag 1974) but male mandibles show sexual shape dimorphism (Kritsky \& Simon 1995, also Chapter 3) related to using the mandibles to grasp the female. Mounting behavior for tiger beetles has been interpreted as a form of contact mate guarding (Kraus \& Lederhouse 1983) and, depending on the species, its duration appears variably influenced by operational sex ratio and environmental factors such as substrate or distance from a suitable oviposition site (Shivashankar \& Pearson 1994), all being factors predicted to influence mating duration by the mate-guarding hypothesis (Alcock 1994, Parker 1970 \& 1974, Thornhill \& Alcock 1983). 
Mate guarding hasused to explain specific observations within a general class of behaviors known as "Post Insemination Associations" (PIAs, Alcock 1994), a type of behavior where mated pairs maintain some form of interaction beyond what is purely necessary for sperm transfer. Reproductive behaviors are assumed shaped to provide an overall increase reproductive success, i.e., selection to maximize fitness (Darwin 1859, Parker 1970). In the face of intra-sexual competition for receptive females, individual males have two stratagies for maximizing their own potential reproductive success: (1) males can invest in inseminating females before other males and (2) males can also attempt to prevent mated females from receiving sperm from other males-a strategy employable both pre- and post-insemination (Parker 1970, Alcock 1994).

Male genital morphology of several species (insect and otherwise) has been associated with the extraction of sperm from the female tract (Simmons 2001, Gallup et al. 2003) as a form of pre-insemination sperm competition. As post-copulatory sperm competition, males of many animal species are known to use several methods to "guard" a female from other males including following the female and chase away any other males that approach (non-contact mate guarding, sensu Alcock 1994) to mechanically occlude the female's genital tract with plugs forms of seminal products or even a detached portion of the male intromittant organ (Parker 1970, Thornhill \& Alcock 1983). A less extreme form of mechanical interference to subsequent mating by a female involves close, physical contact of the pair, usually in a posture similar to or even indistinguishable from actual coitus (Alcock 1994).

Mate guarding is not the only explanation for PIAs between mated pairs and is only expected under conditions of strong male-male competition and last-male sperm 
precedence- where the most recent male to mate dominates the paternity of the brood (Alcock 1994). Alternative circumstances may encourage PIAs, particularly postcopulatory/insemination courtship especially under situations where the female has control over the seminal products and their eventual fate, e.g., the ability to eject a spermatophore post-insemination but pre-fertilization (Alcock 1994, Eberhard 1996).

The genus Omus represents a largely unstudied, basal lineage of tiger beetles endemic to forest-floor environments on the west coast of North America (Pearson \& Vogler 2001, Pearson et al. 2006). These beetles retain multiple ancestral character states such as non-sexually dimorphic mesepisternae on which females lack coupling sulci (Freitag 1974), and inhabiting the forest-floor habitat of sister carabid subfamilies, versus sandy or ashy open substrates seen in more derived cicindeline taxa (Maddison et al. 1999). This basal position of Omus and retention of ancestral character states relative to the majority of the tiger beetles makes them particularly valuable in terms of tracing the evolution of mating behaviors and related morphological adaptations. Additionally, Omus are believed to have atypically long mating durations, with contact anecdotally reported to exceed 16 hours for $O$. dejeanii (Pratt 1939; also see Table 1.3). However these extended compulations have never been formally investigted nor experimentally confirmed for either $O$. dejeanii or any other species of Omus. As such, it remains unknown whether extended amplexus is (a) the rule for the species, (b) typical for the genus or (c) the report of extended mate-contact was an anomalous observation.

The presence of both $O$. audouini and $O$. dejeanii at Powell Butte Nature Park, Portland, Oregon, lends itself to a comparative study of the two species while controlling for geographic factors. In addition to the general intention of establishing average mating 
durations for $\mathrm{Omus,} \mathrm{I} \mathrm{specificially} \mathrm{addressed} \mathrm{three} \mathrm{factors} \mathrm{that} \mathrm{were} \mathrm{hypothesized} \mathrm{to}$ effect mating duration: (1) the time of day mating was initiated, (2) the degree of food deprivation for both sexes and (3) the effects of operational sex ratio.

(A) Time of initiation of mating: I hypothesize that light alone may serve as a cue and mating initiated near the onset of darkness (i.e. evening) will result in longer mating durations than those initiated shortly after the onset of light (i.e. morning). Alternatively, if there is no difference in mating duration, some other cue besides light releases mating termination behavior such as actual environmental stress (heat or disturbance) or a more cryptic cue such as communication between the participants.

(B) Food deprivation: Assuming that (1) mating is not a wholly fixed action pattern behavioral response and that at least one of the participants exercise control over the mating duration, (2) the actual act of insemination requires only a small proportion of the total mating time, and that (3) insemination occurs at some point before the ultimate termination of contact between the partners, I hypothesize that food-deprived beetles will terminate mating behaviors earlier than non-food deprived beetles. Considering the complex relationships among metabolism, morphology and behavior, several other variables will also be examined to account for body size, sexulally selected traits and possible effects of age and capitivity.

(C) Operational sex ratio (OSR): In accordance with the predictions of mate guarding, I hypothesize that matings arranged with a male-biased operational sex ratio will endure longer than matings occurring with an equal or female-biased operational sex ratio. 
Alternatively, if a male-biased OSR does not increase the duration of mating, than the role of extended mating in Omus may not be one of mate guarding.

\section{Collecting and care for 2011 and 2012}

Two species of night stalking tiger beetle, Omus audouini and $O$. dejeanii, were collected using dry pitfall traps and direct searching from 4 May to 30 June 2011 (Julian days 124-181) from forested areas in Powell Butte Nature Park, Portland, Oregon for use in initial baseline mating duration and time of initiation experiments. A total of $83 O$.

audouini (45 female, 38 male) and 22 O. dejeanii (12 female, 10 male) were live captured during the 2011 season.

Areas of Powell Butte Nature Park that yielded successful captures of these target species during 2011 were revisited for live-trapping from 25 June to 27 July 2012 (Julian days 176-208). A total of 113 O. audounini (65 female, 48 male) and 21 O. dejeanii (11 female, 10 male) were live captured during 2012 for use in feeding state and operational sex ratio experiments. See Appendix 1 for specific details regarding laboratory husbandry protocols.

\section{Baseline mating durations and effects of time of initiation}

A. Materials \& methods: Baseline mating durations and effects of time of initiation 1. Materials \& methods: baseline durations and time of initiation, experimental design

To test whether time of initiation affected mating duration, ten pairs of $O$. audouini and ten pairs of $O$. dejeanii were ramndomly selected from a sample of wildcaught individuals to create 20 pairings. All individuals had been maintained in the 
laboratory for a minimum of one week and were of apparent good health; specimens had no identifiable injuries, readily accepted food, and did not display any notably atypical behaviors.

To determine whether there was a circadian influence on mate guarding duration, five pairs from each species were paired at 08:00, one hour after the beginning of the light cycle, and five were paired at 18:00, two hours before the beginning of the dark cycle. Beetles scheduled to mate were fed approximately 24 hours prior to pairing, however it was not documented whether the food was immediately consumed, consumed at a later point, or left uneaten.

Mating enclosures were round, 16 oz delicatessen-style polypropylene containers with ca. 2-3 cm of native soil. A thin layer of native forest floor litter (mostly dried leaf fragments, Douglas-fir cone scales and twigs) was added to provide a degree of environmental structure while not allowing the beetles to be completely obscured. The enclosures were left uncovered during the recording to allow for greater video resolution. The female was introduced to the observation enclosure and allowed to acclimate for a minimum of $45 \mathrm{~min}$, after which time the male was introduced. Behavior was recorded via an IR enabled video camera and reviewed to determine duration of mate guarding. A parallel video recording of initial contact behaviors from the time of introduction to shortly after successful mounting were recorded using a high-definition digital video recorder to capture more subtle details not visible with the IR enabled camera (See Chapter 5 for mating behavior ethogram and evaluation of pre-copulatory struggle).

Body size in insects generally correlates with female fertility (Blanckenhorn 2000, Fairbairn 1997) whereas the mandibles and waists of tiger beetles are known to be 
under sexual selection (Freitag 1974, Kritsky \& Simon 1995). As such, body length (bl), mandible length $(m l)$, and waist width $(w w)$ were measured for females and males as described in Chapter 2 (Figure 2.2 and Table 2.4) to determine whether these morphological features influenced mating duration. After the behavioral experiments were concluded, beetles were euthanized by exposure to cold and stored in $70 \%$ isopropyl alcohol. Considering that younger beetles may mate for a longer or shorter period than older beetles, age of the beetle was approximated arbitrarily as the number of days from 01 June 2011 such that a mating arranged on 20 June 2011 was scored as 20 days.

\section{Materials \& methods: time of initiation experiment, data analysis}

All statistical analyses were undertaken using R (v2.13, R Core development Team 2011) through RStudio V0.96.225 (RStudio 2012). All variables were checked for normality (Shapiro-Wilk test for normality) and datasets being compared checked for homoscedasticity (F-ratio test for equal variance). Differences between: (a) morning and evening matings and (b) the two species were tested for using Welch's two sample t-test.

A multiple linear regression analysis was used to determine whether mating duration could be modeled as a function of a combination of morphological features or seasonality. Variance Inflation Index (VIF) of the predictor variables was used as a measure of possible multi-collinearity (i.e., non-independence among the predictor variables). Backwards selection was used to determine influential predictor variables and construct the best-supported linear model.

Backwards selection begins by constructing a proposed linear model with the single response variable and all of the proposed predictor variables. The coefficients for 
this initial model are estimated. Because the strength of a linear model is initially dependent on the certainty of the slope associated with a given variable being a non-zero value, the predictor variable with the highest $\mathrm{p}$-value above 0.05 is dropped. This process is repeated until there are no more predictor variables with a slope p-value exceeding 0.05 . Secondary criteria for determining the value of a regression model are the $\mathrm{R}^{2}$ estimate of fit and the F-test for whether the majority of variability in the response variable can be attributed to the predictor variables. If there are no predictor variables with a slope $\mathrm{p}$-value $>0.05$, the remaining variables are removed in order of highest $\mathrm{p}$ value one at a time to maximize the $\mathrm{R}^{2}$ and F-statistic.

\section{B. Results: Baseline durations and time of initiation experiment}

1. Results: Mating durations and tests for differences

All continuous variables for all categories of data (species and time) were normally distributed and of equal variance within species for these mating duration datasets. All analyses of mating duration were undertaken in units of hours. Results of a two-sample t-test showed that there was no significant difference $(\alpha=0.5)$ between morning and evening mating durations for either species $($ O. audouini: $\mathrm{t}=-0.94$, d.f. $=8$, $\mathrm{p}$-value $=0.37 ;$ O. dejeanii: $\mathrm{t}=-0.14$, d.f. $=8, \mathrm{p}$-value $=0.89)$, and therefore I pooled the treatments for subsequent analyses. Once pooled, a single pair (O. audouini af69/am86) had a mating duration greater than two standard deviations from the mean (mean $+\mathrm{SD}[2]$ $=11.4+2.9[2]=11.4+5.8=17.2$ hours), was regarded as an outlier and removed from the dataset for statistical tests. 
Full mating results and summary statistics for time of initiation mating durations and associated morphological measurements are given in Table 4.2 and represented in Figure 4.2. Under the described laboratory conditions, Omus audouini mated for an average of $10.6 \pm 1.7$ hours (mean $\pm \mathrm{SD}, \mathrm{n}=9$ observations; excluding the aforementioned outlier) while $O$. dejeanii averaged $29.4 \pm 5.6$ hours (mean $\pm \mathrm{SD}, \mathrm{n}=10$ observations) (Table 4. 3).

2. Results: Multiple regression analysis for baseline mating durations and effects of time of initiation

Multiple regression analysis derived a model for O. audouini where mating duration in hours (durh) was a statistically significant function of three predictor variables: female mandible length $(f m n)$, female waist width $(f w w)$, and male mandible length $(\mathrm{mmn}$ ), estimated as: $d u r h=13.23+11.76 \mathrm{fmn}+19.70 \mathrm{fww}-31.72 \mathrm{mmn}$ (adjusted $\mathrm{R}^{2}=0.87, \mathrm{~F}_{\text {-value }}, 5=19.37$, $\mathrm{p}$-value $\left.=0.004\right)$. Univariate regression analysis indicated that female mandible length $(d u r h \sim f m n)$ was the only significant single predictor of mating duration ( $\alpha=0.05$, Table 4.4).

Multiple regression analysis resulted in a null model for $O$. dejeanii; mating duration was not a statistically significant function of any of the proposed predictor variables $(\alpha=0.05)$. Additionally, none of the proposed predictor variables had a Pearson correlation coefficient greater than 0.23 with respect to mating duration (Table 4.6).

Considering that mating duration may be a function of metabolism and given that metabolism is a function of body mass (Gillooly et al. 2001, Farrell-Gray \& Gotelli 2005), I attempted to express mate guarding duration as a linear function of body mass 
according to a $3 / 4$-power scaling rule, assuming mass is proportional to body length cubed (i.e. $b l^{3}$ ). All resulting regression models were non-significant (Table 4.7).

\section{Effects of food deprivation on mating duration}

A. Materials \& methods: Effects of food deprivation on mating duration

1. Experimental design-Three states were arranged for a sample of wild-caught, laboratory maintained beetles during the summer months of 2012. Beetle were fed ad libitum for one hour then (1) immediately paired for mating (“01"), (2) fasted for ca. 24hours prior to mating ("24") or (3) fasted for ca. 48-hours prior to mating ("48”). This created nine potential experimental treatments. Each treatment (mate combinations of " 01 ", " 24 " and " 48 ") was performed and replicated for $O$. audouini due to the availability of wild-caught specimens. The number of successful captures limited the number of experimental pairings made with $O$. dejeanii to only a sampling of possible combinations (Table 4.8). Prior studies (see "time of initiation experiments" above) showed that $O$. audouini maintain contact mating for ca. 11 hours and $O$. dejeanii ca 30 hours. As such, O. audouini were video recorded for 24 hours and O. dejeanii for 48 hours to ensure full video capture.

Mating duration was defined as the time when the male achieved a stable and persistent mount on the female until the male was fully separated from the female (either spontaneously disengaging or as a consequence of female struggle). All beetles were only used once for any given experimental pairing. Since these animals were collected from the field in mid-season and tiger beetles observe a promiscuous, multiple partner mating system with presumably several reproductive events occurring throughout the 
season, the virginity of the subjects could not be assumed. However for those same reasons, it was assumed that virgin versus non-virgin status would not meaningfully influence the mating duration.

2. Data analysis - A multiple regression analysis similar to that described for the "time of initiation mating duration" experiments was used to construct a regression model to describe mating duration in hours for O. audouini (durh) as a function of one or more variables. Informed by prior results, I chose female mandible length $(f m l)$, female waist width $(f w w)$, male mandible length $(\mathrm{mmn})$ as well as the duration of food deprivation for the female $(f f d)$ and duration of food deprivation for the male $(m f d)$ as predictor variables. Considering that mating duration may be influenced by anatomically-based cryptic female choice for male genital morphology (Eberhard 1996), the male genital length from the proximal bend to the distal tip was also included (aed, see Appendix 2 for genital dissection protocols).

To evaluate possible effects due to captivity or seasonality (age), two additional variables were added for each sex. Captivity/acclimatization ( $f a c c$ and $m a c c$ for females and males, respectively) was measured by number of days the beetle was held in captivity since initial capture and an estimate of beetle age for each sex (fage and mage for females and males, respectively) was counted as the number days since the arbitrarily designated date of 01 July 2012, approximately the beginning of the collecting season.

Because of small sample size and previous null model results for the species, a similar multiple regression analysis was neither possible nor advisable for $O$. dejeanii. Instead, three models were compared in an attempt to model mating duration as a 
function of one or more variables. An initial model used the independent variables of duration of food deprivation for each sex. A second model used length of acclimatization/captivity for each sex. A third and final model used elytra length for each sex an index of body size ( $f e l$ and $m e l$ for female and male elytra length, respectively). Body size was considered possibly meaningful due to the potential for greater energy reserves and, hence, greater resistance to starvation associated with larger individuals. Length of the elytron, measured along the elytral suture, was used as an index of general body length and was chosen since direct measurements of body length were subject to inconsistent values due to flexion of the waist and neck joints. Additionally, female $O$. dejeanii occasionally partially consumed the male post-copulation (a rare event, see Chapter 5) such that only the elytra were available for consistent measurements within the species category.

\section{Results: Effects of food deprivation on mating duration}

Details of pairings and mating durations are given in Table 4.8. For Omus audouini, mating duration in hours was reduced to a non-statistically significant $(\mathrm{p}=$ $0.085, \alpha=0.05$ ) linear function of female age and male acclimatization/captivity (Table 4.9A). Recreating the model from the time of initiation experiment where mating duration was a function of female mandible length, female waist width and male mandible length resulted in a non-significant result $(\mathrm{p}=0.58)$. For $O$. dejeanii, neither food deprivation nor duration in captivity variables were able to construct a model of mating duration that was $p<0.19$. A statistically significant model was constructed for 
O. dejeanii where mating duration was a function of female elytra length and male elytra length ( $\mathrm{p}$-value $=0.05$, Table 4.9B).

\section{Effects of operational sex ratio on mating duration}

\section{A. Materials \& methods: Operational sex-ratio}

1. Experimental design-Three treatments were created to test the effects of operational sex ratio on mating duration: female-male-male (FMM), female-female-male (FFM) and female-male-other (FMX) where "other" was a non-Omus sympatric carabid beetle of approximately the same size scale as a control to test for the possibility of noncompetitive, "witness" effects (Scaphinotus marginatus for O. audouini and $S$. angusticollis for $O$. dejeanii) - i.e, mating duration may be affected by the presence of any beetle, not just a potential mate competitor.

Individuals were uniquely marked on their elytra using a xylene-based paint marker so they could be positively identified on video. To remove the possible effects of feeding state, all specimens were allowed to feed ad libitum for one hour prior to introduction. For female-male-male treatments, males were introduced into the female's home enclosure while females were introduced into the male's home enclosure for female-female-male treatments. Female-male-Scaphinotus arrangements had the male Omus and Scaphinotus introduced into the female enclosure.

Individuals were introduced into enclosure at the same time, within $2 \mathrm{~cm}$ of one another and at least $5 \mathrm{~cm}$ from the occupant (of the opposite sex to the introduced beetles). Prior studies (see "time of initiation experiments" above) showed that $O$. audouini maintain contact mating for ca. 11 hours and $O$. dejeanii ca 30 hours. As such, 
O. audouini were video recorded for 24 hours and $O$. dejeanii for 48 hours to ensure full video capture.

2. Data analysis-Mating duration by treatment within each species was tested for normality using the Shapiro-Wilk test for normality; data were transformed as needed. An ANOVA was used to compare the mating durations for each treatment (FFM, FMM, FMX) and compared to the mean mating times for the food deprivation series (FM, representing equal sex ratio). All statistical analyses were done using R (v2.13, R Core development Team 2011) through RStudio V0.96.225 (RStudio 2012).

Prior research has suggested that $O m u s$ sex ratios in natural populations are likely at or near 1:1 (Leffler \& Nelson 1986). A $\chi^{2}$ test was used to test the null hypothesis that the mean ratio of females-to-males was not significantly different among the field captured individuals. All beetles were captured using unbaited pitfall traps made out of plastic drinking cups and assumed to be neutral, neither attracting nor repelling the target beetles. Capture numbers includes individuals caught in dry traps as well as "wet" traps, i.e. traps with a solution of water and a few drops of unscented, biodegradable detergent. Capture numbers were tested under the assumption that there was no trap bias for either sex, i.e., both sexes were equally likely to be captured. Additionally, I assumed that captures were independent events and that previous captures were independent of subsequent captures. These numbers do not include speculative or inferred captures of individuals lost to intratrap predation — beetles eaten by other occupants of the trap such as other predatory arthropods or shrews as evidenced by specimen fragments (e.g. legs and disembodied fragments of cuticle). 


\section{B. Results: Effects of operational sex ratio on mating duration}

Details of pairings and mating durations are provided in Table 4.10. The mating durations for each species did not depart from normality, thus did not require transformations prior to testing $(O$. audouini: $\mathrm{n}=24, \mathrm{~W}=0.96, \mathrm{p}$-value $=0.46 ; O$. dejeanii: $\mathrm{n}=12, \mathrm{~W}=0.95, \mathrm{p}$-value $=0.63)$. No significant differences were detected among the treatment groups for either $O$. audouini $(\mathrm{F}-\mathrm{value}=0.65$, p-value $=0.43)$ or $O$. dejeanii $($ F-value $=0.23$, p-value $=0.64)($ Table 4.11, Figure 4.3 $)$.

Specimen capture results are given in Table 4.12. While O. audouini were, on average, present in slightly female-biased numbers, $O$. dejeanii sex-ratio was exactly equal when averaged across the three years. A $\chi^{2}$-test was highly insignificant for both species $(\alpha=0.05$, $p$-value $>0.50$; Table 4.13) indicating that the null hypothesis should not be rejected and the mean sex ratios of natural populations may be regarded as equal.

The behaviors observed among individuals during the experiment could be characterized as (1) neutral, (2) aggressive or (3) reproductive. Neutral responses were characterized by a beetle not responding to another beetle as different from any other part of the environment, often walking over the beetle or, at most, briefly examining the other beetle with antennae. Aggressive responses were displays of gaping mandibles and attempts to bite the other beetle, often in response to contact. Reproductive responses were attempts to mount another beetle and only seen in males. Under male-biased conditions, the unpaired male would frequently mount the mating pair foring a "triplex" of beetles. Triplexing would endure for a minute at most, with the top beetle extending his genitals and probing the caudal regions of the mating pair before disengaging. 
Female-female interactions were either neutral or aggressive, females either ignoring each other or biting and displaying gaped mandibles, regardless of whether they were mounted. Despite evidence that $O$. dejeanii will prey on Scaphinotus spp in captivity (R.K.R. pers. observ.), interactions between Omus and the witness Scaphinotus were neutral for all observations, one neither attacking nor mounting the other.

\section{Discussion}

None of the measured variables accounted for variation in mating duration for either species between the two years (i.e. time of initiation, 3/4-power scaling, food deprivation, morphological characters and time-based characters such as age or duration in captivity). Additionally, none of the experimental treatments resulted in statistically significant differences between or among the categories (time of initiation, food deprivation or operational sex ratio).

Pooling the 2011 time of initiation experimental observations (morning versus evening initiation of mating), it was established that $O$. audouini have a mean mating duration of $10.6 \pm 1.7$ hours while $O$. dejeanii have a mean mating duration of $29.4 \pm 5.6$ hours, nearly double the previously reported (and anecdotal) duration of ca. 16 hours (Pratt 1939).

The experimental results inconsistently identified morphological factors, age/temporal factors or none of the examined factors as having significant effects on mating duration. These discrepancies may be due to the complexity of the behavior, with a proper analysis likely requiring a greater sample size. Alternatively, these factors may legitimately not affect mating duration. Further, there may remain unknown aspects of 
the physiology and behavior of this basal group of tiger beetles that determines for how long these beetles copulate and maintain contact.

The mating behavior displayed by these tiger beetles, where males remain mounted on females for a time period seemingly beyond what would be required for sperm transfer, has been interpreted as a form of contact mate guarding (Kraus \& Lederhouse 1983). Mate guarding postulates that this behavior results from selective pressures stemming from strong intrasexual competition, for example species with lastmale sperm precedence and, particularly, male-biased operational sex ratios - the male able to inseminate closest to the time of egg release has the greatest genetic representation in the subsequent brood (Parker 1974, Alcock 1994).

Little to no work has been published regarding the population biology of Omus, either population densities or sex ratios of natural populations. A single study (Leffler \& Nelson 1986) as well as my own experience (Table $4.12 \& 4.13$ ) supports the proposition that both $O$. audouini and $O$. dejeanii exist in equal or near equal sex ratios in natural populations. Additionally, the greater complexity of the forest floor habitat relative to more exposed environments may buffer temporary sex ratio biases by making it more difficult for an individual to encounter another conspecific, regardless of sex. Given those circumstances, it may be possible that the long mating duration of Omus relative to other tiger beetle genera is due to factors other than mate guarding. As an alternative, it may be that mating in Omus is better explained as a manifestation of cryptic female choice and interpreted as a form of syn-copulatory courtship.

Observations of mating in Cicindela species have reported periods during which the male will remain mounted on the female post-intromission but without genital 
contact, an act strongly suggestive of mate guarding, or will repeatedly insert and then fully withdraw his aedeagus while mounted, presumably as a way to extract seminal products from previous partners before depositing his own as a form of direct sperm competition (Willis 1967, Freitag et al. 1980, Kraus \& Lederhouse 1983, Fielding \& Knisley 1995). This was not the case for Omus - the male intromittent organ appeared to remain inserted in the female throughout the duration of the mount for all observations of both $O$. audouini and $O$. dejeanii. Multiple thrust types occured for both species of Omus (see mating behavior ethogram, Chapter 3, Table 3.1) but none of them appeared to involve full withdrawal of the male organ. While the different thrust types may be involved in contacting different parts of the female genital tract and displace previously deposited seminal products, without withdrawal of the male organ there does not seem to be a mechanism to actually remove sperm from the female genital tract altogether.

The females of some species of tiger beetles - as well as those of other carabid beetle species - have demonstrated the ability to eject seminal products post-copulation (Rodriguez 1998 \& 1999, Takami 2007). In addition, the ability of the female to manipulate the presence and use of deposited seminal products is documented in many insect groups (Eberhard 1996, Simmons 2001, Klowden 2007). The combination of (1) extended mating contact, (2) persistent genital-genital contact (including at least partial intromission), (3) an absence of significant difference in duration due to varying sex ratios, and (4) the possibility of female ejection of unwanted sperm, allows us to reframe the behavior as an extended and subtle syn-copulatory courtship, potentially an extension of female choice sexual selection (Eberhard 1996). 
Unfortunately, detailed studies of mating behavior are largely lacking for the basal members of Cicindelinae and rare among closely related sister clades such as Rhysodinae, Scaritinae and Paussinae (the "CRSP quartet", Maddison et al 1999). It is unknown whether the character state of a relatively extended mating duration is a retained ancestral trait for Omus. Conceivably, the long mating duration could have been adapted to a shorter duration when tiger beetle habitats diversified from forests to those of stream banks and salt flats. Rather than the sheltered habitat of densely canopied forests and thick underbrush, the new, highly exposed and arid habitats brought not only an increased risk of visual detection by same-sex competitors but also an increased risk of exposure and predation.

Considering the innately different "agendas" of females and males (quality versus quantity of offspring, respectively, i.e. Bateman 1948, Parker et al. 1972, Trivers 1972, also Eberhard 1996, Birkhead 2000, Simmons 2001), it is likely that female-choice mechanisms such as the demands of courtship are commonplace. However there is a need to first verify whether female choice or male-male competition can be invoked as an explanation for any given behavior as well as partition the relative influence of each force in shaping and maintaining the behavior. The confirmation of one does not preclude the influence of the other since co-evolution is known among interdependent species (e.g. Roughgarden 1976, Connell 1980) and evolutionary "arms races" are not unheard of between sexes within a species (Eberhard 1996, Birkhead 2000, Simmons 2001). A combination of comparative studies of mating behaviors across multiple taxa accounting for ecological and evolutionary differences as well as continued examination of the genus Omus, including population densities and hypotheses of phylogenetic relationships, 
would be invaluable towards teasing apart the respective influence of female-choice relative to intrasexual (male-male) competition. 


\section{Chapter 5: Conclusions}

\section{Summary and recapitulation of chapter results}

I sought to address questions related to the ecomorphology and mating behaviors of two species of Omus. In Chapter 2, I examined the general morphology of Omus and, because of their use as feeding organs and secondary sexual organs, the specific morphology of the mandibles. Through univariate and multivariate analyses, I found that the two species examined, Omus audouini and $O$. dejeanii, are essentially isometrically scaled versions of each other and both species showing similar female-biased dimorphism in overall morphology. Two exceptions were the characters of mandible length and antennal length.

Mandible length was both proportionally and absolutely male-biased in $O$. audouini. Mandibles length in $O$. dejeanii was not sexually dimorphic in length on an absolute scale but, because body size was larger in females, the male mandibles were proportionally larger relative to body size. Additionally, there was no sexual dimorphism in the absolute length of antennae for either species, but accounting for larger body sizes in females, males of both species have proportionally larger antennae than females.

A comparative geometric morphometric analysis of mandibles between the two species and the sexes found significant and prominent morphological differences. The species-level differences were most noticable on the proximal region of the mandible and involved the robustness and angles of the terebral teeth. I found that $O$. audouini have relatively slender and anteriorly oriented teeth ("shearing" teeth) while $O$. dejeanii have more robust and medially oriented teeth ("cracking" teeth). I infer these differences to relate to the natural prey base of the two species and the "shearing" teeth of $O$. audouini 
are adapted for processing soft, weakly- or un-sclerotized prey items such as larva or soft-bodied arthropods of suitable size. In contrast, the "cracking" teeth of $O$. dejeanii may be an adaptation for more durable prey such as adult ground beetles, millipedes and, opportunistically, larger soft-bodied prey inaccessible to $O$. audouini.

Sex-level dimorphisms were similar in nature for both species and primarily involved the curvature of the apical tooth. Females of both species had relatively shorter and more curved mandibles while males had longer and straighter mandibles. The straighter apicle incisors are likely a result of sexual selection, the male mounting the female and grasping her around the waist.

Chapter 3 provided an ethogram of mating behaviors for the two species of Omus. I found that the mating behavior of Omus was distinct relative to non-Omus tiger beetles and also distinctive between the two Omus. Mating in Omus does not appear to involve pre-copulatory courtship and the male attempts to mount the female immediately on contact. Upon mounting, the male uses his antenna to vigoriously stroke the head and antenna of the female. He then extends and inserts his genitals and copulation takes place. While mounted, the males engges in bouts of thrusts between long periods of relative inactivity from the male while the female moves about engaging in typical behaviors.

The two species of Omus displayed different types of intromittant thrusts. Multiple thrust types are known in other genera of tiger beetle and are associated with sperm competition — where the male attempts to displace pre-existing seminal productsas well as intersexual competition such that the male is attempting to influence placement of his own seminal products within the female reproductive tract. 
Pre-copulatory struggle was seen in both sexes on being mounted by a conspecific male. This observation invites further investigation whether the pre-copulatory struggle is a female-choice mechanism or an anti-predator defense behavior present in both sexes that has secondarily been adopted by females to influence mate choice. Finally, two incidents of post-copulatory sexual cannibalism were documented by a single female $O$. dejeanii, a previously undocumented behavior for the genus. While likely an artifact of the laboratory conditions, cannibalism and intraguild predation within natural Omus communities may be a small but significant factor in their popoulation ecology.

Chapter 4 addressed sexual size dimorphism and the effects of experimental manipulations on mating behaviors in Omus. Tiger beetles were found to maintain isometric sexual size dimorphism, the females being on average $8 \%$ larger than the males across a broard range of body sizes, habitats and mating durations. Experimental manipulations examined the effects of time of day mating was initiated, food deprivation and operational sex ratio on mating duration. Aside from determining the baseline mating durations for the two species under laboratory conditions, manipulative experiments were inconclusive and inconsistent. The average mating duration (mean \pm SD) for $O$. audouini was $10.6 \pm 1.8$ hours ( $\mathrm{n}=9$ observations; excluding the a single outlier) and $O$. dejeanii was $29.4 \pm 5.6$ hours ( $=10$ observations). These durations were not consistently correlated with the examined gross morphological characteristics and neither did they respond to manipulations of time of initiation, food availability or operational sex ratio.

\section{Synthesis of results among chapters}


This research has contributed to our understanding of the ecology and behavior of two species of a largely unstudied genus. In addition to the conclusions drawn from within the individual chapters, these results may be further synthesized into inferences regarding previously unknown features of the natural history of Omus.

\section{A. Prey base}

The natural prey base of Omus has been a matter of speculation for some time (e.g. LaBonte \& Johnson 1988). As a genus, Omus is assumed to be a generalist predator of terrestrial invertebrates. However niche partition theory predicts that syntopic congenerics would adapt to decrease direct competition for resources, particularly if the original resource is limited. The genus of Omus has low recognized species diversity, particularly in comparison to other tiger beetle genera, and only rarely allows for comparisons among syntopic species.

Niche partitioning between Omus audouini and O. dejeanii is supported by the observed Hutchinsonian ratios in body sizes (x1.3) and mandible lengths (x1.5) as well as shape differences in mandibles. All currently described tiger beetles are predatory in addition to possessing distinctively large, curved mandibles that are used in both prey capture and, in males, as secondary sexual organs. An extended investigation regarding the specific geometry of the mandibles of Cicindelines in addition to field studies regarding their natural prey base will reveal whether mandibular morphology consistently correlates with and, hence, likely defines prey.

\section{B. Copulatory courtship and female-choice in mating}


The results cast doubts on whether the distinctive mating behavior of tiger beetles can be universally regarded as mate guarding as an extension of intrasexual (male-male) competition. Changes in the operational sex ratio did not affect Omus mating durations. With Omus, genital contact involved at least partial insertion of the aedagus into the female tract continuously throughout the duration of the mount for both $O$. audouini and O. dejeanii. Persistent genital contact has not been reported for other genera of tiger beetle but, to the contrary, periods of genital withdrawal while mounted have been described. Continuious genital contact, while blocking access to competitors, may also be seen as part of a communitation between the participants, i.e. a mechanism of syncopulatory courtship.

Considering that genital insertion is apparently not necessary to block the female tract from male competitors and the operational sex ratio did not result in significant differences among sex ratio treatments, we may hypothesize that the primary pressures shaping Omus mating behaviors is something other than males guarding an inseminated female from other males. Given the observations of female control over seminal products (as described in Chapter 3), extended mating duration with genital contact and no statistically significant effect on mating duration due to sex ratios, the mating behavior of Omus may be explicable as syn-copulatory courtship rather than post-insemination mate guarding. This hypothesis of syn-copulatory courtship is supported by the observed repeated occurrences of behaviors associated with general insect courtship such as tactile cues in the form of antenna stroking (e.g. Eberhard 1994) during mating, particularly in response to female struggle. Also, struggle behavior from mounted beetles, both males and females, was apparently repressed by antennal tactile cues from the mounting beetle, 
i.e., the male stroking and drumming the mounted beetle's head and antennae with his own antennae.

It is well supported that Omus represent basal members of Cicindelinae (Vogler \& Pearson 1996, Maddison et al. 1999). Unfortunately, without behavioral information relative to sister Carabid clades, these character states of extended mating duration and persistent genital contact may either be derived autapomorphies for Omus or ancestral and retained in Omus but lost in all other lineages. Additionally, other basal or forestdwelling genera of tiger beetles, e.g. Amblycheila and Picnochile of North and South America, have received little-to-no attention with the exception of Pseudoxchila, so information to be used in comparative studies remains too scarce to be definitive towards inferring the evolutionary history or potential selective advantages of the mating behaviors described for Omus.

Regardless of whether ancestral or derived, it is reasonable to speculate that the complex and sheltered habitat of the forest floor played no small role in shaping the current forms of mating behaviors. As the tiger beetles diversified out from forest floor habitats, they were subjected to more exposed circumstances; exposure to abiotic environmental factors, exposure to predators and exposure to competitors - both interspecific and intraspecific. As a consequence, extending mating durations with the associated handicaps of burdened movement and larger visual profile would be a detriment and selection would favor strategies with a lower time investment to exchange and protect gametic investment. In contrast, the forest floor offers a multitude of microhabitats and refugia that potentially could have allowed the development of the 
more involved mating behavior as seen in Omus, either as a novel development or an amplification of pre-existing behavioral patterns.

While habitat can be invoked as an explaination for the Omus-type mating behaviors, the question of what delimits the mating duration or explains the difference in duration between the two species is yet to be resolved. Mating duration could not be explained as a function of body size or food deprivation and neither time of initiation (testing for external light cues) nor intrasexual competition appeared to have a significant effect on duration for the examined species. There still remains the possibility of humidity and temperature as probably influencing factors, a decrease in humidity or increase in thermal stress likely to result in shorter mating durations.

Additionally, the differences between the two species invite a larger investigation as to whether habitat specificity relates to mating duration. While all known Omus are endemic to forests of the West-coast of North America, the specific habitat-types range from meadows bordering established forests (O. audouini), relatively dry oak-pine forests (O. submetallicus and O. cazerii) to dense coniferous forests with a heavy layer of leaf mould and woody debris as substrate (O. californicus and O. dejeanii) (Pearson et al. 2006). The genus also exists along elevations ranging from coastal (O. dejeanii has been reported along Pacific beach debris, Pearson et al. 2006) to high-elevation (O. cazierii known only around Mt. Ashland in southwest Oregon, USA and found at elevations >1000 m; van den Berghe 1994 and R.K.R. pers. observ.). An evaluation including several species within the genus would allow for examination of the effects of habitat preferences on mating duration with the prediction that more exposure-tolerant species (i.e. O. audouini, O. cazerii and O. submetallicus) will exhibit shorter mating durations as 
an adaptation to their environment while $O$. californicus and $O$. dejeanii, known to predominately occupy well-sheltered habitats, would show the longest mating durations.

\section{Further avenues of study and conclusion}

The research described, both performed and proposed has contributed to our understanding of not only the genus Omus but the to general fields of reproductive biology and ecomorphology. There still remain several avenues of research to complement and expand on the themes touched on in this dissertation.

The prey bases for natural populations of Omus still need to be determined and niche partitioning among sympatric species confirmed. As prominent predators of North American West-coast forest floors, an understanding of the community interactions and population dynamics of Omus would, in turn, assist in a more detailed understanding of the forest-floor community as a whole.

Related to mandibular mandibular anatomy, a quantification of mechanical costs and benefits of the mandibular architecture would be of value towards evolutionary trends in the diet and feeding apparatus of Coleoptera as well as other mandibulate insects. Specifically of interest would be the compromises made for shearing versus crushing forces in respect to the terebral tooth geometry. Additionally, developmental processes governing mandibular sexual differentiation would be of value in studies related to sex-linked gene expression and the physiology, organization and time of critical events of holometabolous insect metamorphosis, e.g., at what point do the mandibles begin to sexually differentiate during pupation and what mechanisms allocate resources to or from the mandibles? 
While Omus as a genus has low species representation, populations are common enough to merit attention purely from a community ecology perspective. Evolutionarily, they are uniquely placed at or near the base of a prominent, divergent and diverse lineage of beetles allowing for the tracing of character states. Unfortunately, much about Omus remains unknown. While not exhaustive, the body of work presented here serves as a valuable continuation of prior studies (e.g. Pratt 1939, Cazier 1942, Leffler 1979, van den Berghe 1990) that, hopefully, will in turn inspire future study of Night-stalking tiger beetles. 


\section{$\underline{\text { Tables }}$}

Table 1.1: Global species biodiversity estimates and percentage of that diversity due to insects.

\begin{tabular}{lccc} 
Taxa & Bailie et al (2004) & Chapman (2009) & Vie et al (2009) \\
\hline Chodates/vertebrates & 57739 & 64788 & 61259 \\
Invertebrates & 1190200 & 1359365 & 1232384 \\
TOTAL animal species & 1247939 & 1424153 & 1293643 \\
Insecta & 950000 & 1000000 & 950000 \\
$\%$ insects & $76 \%$ & $70 \%$ & $73 \%$
\end{tabular}


Table 1.2: Stages in which reproductive success can be manipulated and examples of mechanisms. Mechanisms that may be strongly female controlled are in bold.

Information assembled from: Brikhead 2000; Eberhard 1991, 1994, 1996; Thornhill \& Alcock 1983.

\begin{tabular}{|c|c|}
\hline Stage & Mechanism \\
\hline Mate encountering & Either partner may hide/avoid suitors \\
\hline Courtship & Either partner may reject mate advances \\
\hline \multirow[t]{2}{*}{ Copulation } & Either partner may break off coupling \\
\hline & $\begin{array}{l}\text { Male may bodily exclude other males (i.e. syn-copulatory mate } \\
\text { guarding) }\end{array}$ \\
\hline \multirow[t]{4}{*}{ Intromission } & $\begin{array}{l}\text { Females may sequester, exclude or eject sperm from genital } \\
\text { tract }\end{array}$ \\
\hline & $\begin{array}{l}\text { Male may exclude other sperm (e.g. plugs, post-copulatory mate } \\
\text { guarding) }\end{array}$ \\
\hline & Other males may extract existing sperm \\
\hline & Other males may swamp/dilute competitor sperm \\
\hline \multirow[t]{4}{*}{ Fertilization } & Non-receptive/un-prepared womb/ova \\
\hline & Implantation may not occur \\
\hline & Zygote may be destroyed \\
\hline & Egg/fetus may be aborted \\
\hline & Offspring or clutch may be destroyed (by male or female) \\
\hline Raising offspring & Abandonment or lack of care to offspring (by male or female) \\
\hline
\end{tabular}


Table 1.3: Carabid beetles known to engage in dorso-ventral mating. "Tiger?" indicates whether the beetle is classified as a tiger beetle. Contact duration includes pre-, syn- and post-copulatory stages of mating behavior when the male is in a mounted position on the female independent of genital contact.

\begin{tabular}{|c|c|c|c|c|}
\hline Genus & Species & Tiger? & Contact duration & Source \\
\hline Brachinus & pallidus & $\mathrm{n}$ & $>1$ hour $^{\mathrm{a}}$ & Erwin 1967 \\
\hline Carabus & multiple & $\mathrm{n}$ & $163 \min ^{\mathrm{b}}$ & $\begin{array}{l}\text { Takami 2002, } \\
\text { Takami \& Suzuki } 2005\end{array}$ \\
\hline Cicindela & multiple & $\mathrm{y}$ & $6-70 \mathrm{~min}$ & $\begin{array}{l}\text { Kraus \& Lederhouse (1983) } \\
\text { Shivashankar \& Pearson } \\
\text { (1994) } \\
\text { Fielding \& Knislev (1995) }\end{array}$ \\
\hline Pseudoxychila & tarsalis & $\mathrm{y}$ & $13 \min ^{\mathrm{c}}$ & $\begin{array}{l}\text { Fielding \& Knisley (1995) } \\
\text { Rodriguez (1998) }\end{array}$ \\
\hline Pseudoxychila & bipustulata & $\mathrm{y}$ & ca $290 \mathrm{~min}^{\mathrm{d}}$ & Rodriguez (1998) \\
\hline Oтия & dejeanii & $\mathrm{y}$ & $>16$ hours $^{\mathrm{e}}$ & Pratt (1939) \\
\hline Ohomopterus & multiple & $\mathrm{n}$ & $29-275 \mathrm{~min}$ & Takami \& Sota 2007 \\
\hline Zacotus & matthewsii & $\mathrm{n}$ & unknown & Personal observation by RKR \\
\hline
\end{tabular}

a "Actual intromission lasts only a few seconds, but the male remains mounted on the female for an hour or more if not disturbed." (Erwin 1967)

${ }^{\mathrm{b}}$ Takami (2002) reported a mean copulatory duration of 97 minutes and a mean postcopulatory contact duration of 66 minutes. Mean pre-copulatory contact was $<1$ minute. ${ }^{\mathrm{c}}$ Rodriguez (1998) $12.9 \pm 1.5,0.3-93.3 \mathrm{~min}, \mathrm{n}=153$.

${ }^{\mathrm{d}}$ Exact mean value not given

e "I do not know how long the pair had been mating when first found or how long they would have remained in that state if undisturbed, but the total observed period of mating was sixteen hours and thirteen minutes." (Pratt, 1939) 
Table 2.1: Descriptions of morphometric features and abbreviations use in analysis. All measurements were made in millimeters.

Body length (BL): $\quad$ Measured from anterior medial point of labrum to the distal medial point of the elytra

Antennal length (AN): $\quad$ Measured from the base of the scape to the tip of the distal segment

Waist width (WS): $\quad$ Measured as minimum distance between lateral points of the waist perpendicular to a ventral-dorsal plane

Pronotum length (PL): $\quad$ Measured as the maximum distance along the medial axis

Pronotum width (PW): $\quad$ Measured as the maximum distance found perpendicular to a ventral-dorsal plane

Elytra length (EL): $\quad$ Measured as the maximum distance along the medial axis (elytral suture)

Elytra width (EW): $\quad$ Measured as the maximum distance found perpendicular to a ventral-dorsal plane

Length of femur (FE): $\quad$ Length from proximal medial base of trochanter to femur distal medial point of femur

Length of tibia (TI): $\quad$ Maximum distance of the structure measured from proximal point along the lateral border to most distal point along the lateral border.

Length of mandible $\quad$ Measured as the chord distance from the lateral point of $(\mathrm{MN})$ : articulation to the distal tip of the left mandible Head width (HW): $\quad$ Measured as the maximum width of the head (eyes) found perpendicular to a ventral-dorsal plane 
Table 2.2: Summary statistics for individual characters. (A) O. audouini, female; (B) $O$. audouini, male, (C) O. dejeanii, female; (D) O. dejeanii, male.

(A) O. audouini, female; $\mathrm{n}=70$

\begin{tabular}{lrrc} 
Character & Median & Mean \pm SD & (Low-High) \\
\hline Body length: & 16.00 & $16.02 \pm 0.66$ & $(14.00-17.50)$ \\
Antenna length: & 8.60 & $8.58 \pm 0.36$ & $(7.80-9.40)$ \\
Waist width: & 3.15 & $3.16 \pm 0.12$ & $(2.90-3.50)$ \\
Pronotum length: & 3.65 & $3.65 \pm 0.13$ & $(3.35-4.05)$ \\
Pronotum width: & 4.30 & $4.30 \pm 0.16$ & $(3.75-4.55)$ \\
Elytra length: & 9.30 & $9.30 \pm 0.37$ & $(8.20-10.00)$ \\
Elytra width: & 6.00 & $5.94 \pm 0.40$ & $(3.35-6.45)$ \\
Length of femur: & 5.00 & $5.00 \pm 0.18$ & $(4.50-5.30)$ \\
Length of tibia: & 4.90 & $4.89 \pm 0.20$ & $(4.30-5.30)$ \\
Mandible length: & 2.80 & $2.79 \pm 0.10$ & $(2.60-3.15)$ \\
Head width: & 3.90 & $3.85 \pm 0.13$ & $(3.40-4.15)$
\end{tabular}

(B) O. audouini, male; $\mathrm{n}=70$

$\begin{array}{lrrc}\text { Character } & \text { Median } & \text { Mean } \pm \text { SD } & (\text { Low-High }) \\ \text { Body length: } & 14.90 & 14.85 \pm 0.60 & (13.40-16.10) \\ \text { Antenna length: } & 8.70 & 8.70 \pm 0.33 & (7.90-9.40) \\ \text { Waist width: } & 2.95 & 2.92 \pm 0.11 & (2.65-3.15) \\ \text { Pronotum length: } & 3.55 & 3.52 \pm 0.15 & (3.05-3.80) \\ \text { Pronotum width: } & 3.95 & 3.94 \pm 0.14 & (3.55-4.25) \\ \text { Elytra length: } & 8.55 & 8.56 \pm 0.32 & (7.95-9.25) \\ \text { Elytra width: } & 5.30 & 5.34 \pm 0.22 & (4.80-5.90) \\ \text { Length of femur: } & 4.75 & 4.77 \pm 0.17 & (4.40-5.20) \\ \text { Length of tibia: } & 4.80 & 4.79 \pm 0.19 & (4.30-5.20) \\ \text { Mandible length: } & 3.10 & 3.11 \pm 0.09 & (2.90-3.35) \\ \text { Head width: } & 3.60 & 3.61 \pm 0.11 & (3.40-3.95)\end{array}$

(C) O. dejeanii, female; $\mathrm{n}=43$

Character

Body length:

Antenna length:

Waist width:

Pronotum length:

Pronotum width:

Elytra length:

Elytra width:

Length of femur:

Length of tibia:

Mandible length:

Head width:
Median

20.30

10.90

3.80

4.50

6.50

11.45

8.00

6.30

6.40

4.30

5.25
Mean $\pm \mathrm{SD}$

$20.36 \pm 0.70$

$10.82 \pm 0.36$

$3.82 \pm 0.13$

$4.49 \pm 0.14$

$6.51 \pm 0.19$

$11.43 \pm 0.39$

$7.97 \pm 0.22$

$6.28 \pm 0.18$

$6.37 \pm 0.21$

$4.32 \pm 0.13$

$5.23 \pm 0.16$
(Low-High)

$(18.60-21.90)$

$(10.10-11.60)$

$(3.50-4.10)$

$(4.05-4.70)$

$(6.10-6.90)$

$(10.35-12.20)$

$(7.50-8.45)$

$(5.80-6.60)$

$(5.80-6.80)$

$(4.06-4.70)$

$(4.85-5.50)$ 
(Table 2.2 cont.)

(D) O. dejeanii, male; $\mathrm{n}=50$

Character

Median $\quad$ Mean \pm SD

(Low-High)

Body length:

18.65

$18.75 \pm 0.74$

$(17.20-21.20)$

Antenna length:

$10.85 \quad 10.80 \pm 0.36$

$(10.10-11.50)$

Waist width:

$3.53 \quad 3.54 \pm 0.09$

$(3.30-3.75)$

Pronotum length:

$4.40 \quad 4.36 \pm 0.15$

$(3.95-4.60)$

Pronotum width:

$5.90 \quad 5.88 \pm 0.21$

$(5.55-6.55)$

Elytra length:

10.55

$10.54 \pm 0.31$

$(10.00-11.50)$

Elytra width:

7.20

$7.19 \pm 0.22$

$(6.70-7.90)$

Length of femur:

Length of tibia:

Mandible length:

$5.90 \quad 5.90 \pm 0.17$

$(5.60-6.30)$

6.20

$6.17 \pm 0.19$

$(5.70-6.60)$

Head width:

4.30

$4.31 \pm 0.18$

$(4.00-4.80)$

4.78

$4.79 \pm 0.16$

$(4.35-5.20)$ 
Table 2.3: Body length and mandible ratios for species and sexes. (A) Species comparison, (B) O. audouini sex comparison, (C) O. dejeanii sex comparison. Body length $=\mathrm{BL}$, Mandible length $=\mathrm{MN}$.

(A) Species comparison Species Mean BL Mean MN

O. audouini 15.4 2.9

O. dejeanii 19.5

Ratio

(B) O. audouini sex comparison

\begin{tabular}{lcc} 
Sex & Mean BL & Mean MN \\
\hline Female & 16.0 & 2.79 \\
Male & 14.9 & 3.11 \\
Ratio & 1.1 & 1.1
\end{tabular}

(C) O. dejeanii sex comparison

\begin{tabular}{lcc} 
Sex & Mean BL & Mean MN \\
\hline Female & 20.3 & 4.32 \\
Male & 18.7 & 4.30 \\
\hline Ratio & 1.1 & 1.0
\end{tabular}


Table 2.4: PC scores (eigenvectors) and eigenvalues of PCA of overall body morphology for both species of Omus. See Figure 2.3 for corresponding plot of PCA.

$\begin{array}{lcr}\text { Feature } & \text { PC1 } & \text { PC2 } \\ \text { Body Length } & 0.30 & -0.19 \\ \text { Antenna Length } & 0.29 & 0.45 \\ \text { Waist Width } & 0.30 & -0.37 \\ \text { Pronotum Length } & 0.30 & 0.08 \\ \text { Pronotum Width } & 0.31 & -0.02 \\ \text { Elytra Length } & 0.30 & -0.34 \\ \text { Elytra Width } & 0.30 & -0.26 \\ \text { Femur Length } & 0.31 & -0.05 \\ \text { Tibia Length } & 0.30 & 0.19 \\ \text { Mandible Length } & 0.29 & 0.62 \\ \text { Head Width } & 0.31 & -0.07 \\ \text { SD } & 3.22 & 0.56 \\ \text { Eigenvalue } & 10.38 & 0.31 \\ \text { \% Var } & 94 \% & 3 \% \\ \text { Cum \% } & 94 \% & 97 \%\end{array}$


Table 2.5: PC scores (eigenvectors) and eigenvalues of PCA of overall body morphology for individual species of Omus. (A) Omus audouini, (B) Omus dejeanii. See Figure 2.4 for corresponding plot of PCA.

\begin{tabular}{lcr} 
(A) Omus audouini & \\
Feature & PC1 & PC2 \\
\hline Body Length & -0.32 & 0.00 \\
Antenna Length & -0.13 & 0.65 \\
Waist Width & -0.35 & -0.10 \\
Pronotum Length & -0.30 & 0.16 \\
Pronotum Width & -0.35 & -0.12 \\
Elytra Length & -0.35 & -0.07 \\
Elytra Width & -0.30 & -0.16 \\
Femur Length & -0.34 & 0.16 \\
Tibia Length & -0.26 & 0.32 \\
Mandible Length & 0.17 & 0.61 \\
Head Width & -0.35 & -0.04 \\
SD & 2.66 & 1.28 \\
Eigenvalue & 7.10 & 1.64 \\
\% Var & $65 \%$ & $15 \%$ \\
Cum \% & $65 \%$ & $79 \%$
\end{tabular}

(B) Omus dejeanii

\begin{tabular}{lcr} 
Feature & PC1 & PC2 \\
\hline Body Length & -0.32 & -0.14 \\
Antenna Length & -0.16 & 0.63 \\
Waist Width & -0.33 & -0.11 \\
Pronotum Length & -0.28 & 0.29 \\
Pronotum Width & -0.34 & -0.18 \\
Elytra Length & -0.34 & -0.15 \\
Elytra Width & -0.33 & -0.24 \\
Femur Length & -0.34 & -0.01 \\
Tibia Length & -0.29 & 0.18 \\
Mandible Length & -0.16 & 0.57 \\
Head Width & -0.35 & -0.12 \\
SD & 2.79 & 1.14 \\
Eigenvalue & 7.76 & 1.29 \\
\% Var & $71 \%$ & $12 \%$ \\
Cum \% & $71 \%$ & $82 \%$
\end{tabular}


Table 2.6: Reduced major axis linear regression results for allometry of antenna length and mandible length relative to body length. Regression formula is in the form of: $\mathrm{Y}=$ $\mathrm{X} \beta+a$, where $\beta=$ slope and $a=$ intercept. Slope and intercept are given with $2.5 \%-$ $97.5 \%$ confidence intervals. (A) Regression of $\ln$ (Antenna length) onto $\ln$ (Body length), (B) Regression of $\ln$ (Mandible length) onto $\ln$ (Body length).

(A) Regression of $\ln$ (Antenna length) onto $\ln$ (Body length)

$\begin{array}{lcccccc}\text { Species } & \mathrm{Sex} & \mathrm{N} & \mathrm{r}^{2} & \mathrm{p} \text {-value } & \beta(2.5 \%-97.5 \%) & a(2.5 \%-97.5 \%) \\ \text { O. audouini } & \mathrm{F} & 70 & 0.45 & <0.001 & 0.99(0.83-1.18) & 0.64(0.23-0.99) \\ \text { O. audouini } & \mathrm{M} & 70 & 0.34 & <0.001 & 1.08(0.89-1.31) & 0.36(-0.14-0.78) \\ \text { O. dejeanii } & \mathrm{F} & 43 & 0.12 & 0.022 & 1.03(0.77-1.38) & 0.56(-0.27-1.18) \\ \text { O. dejeanii } & \mathrm{M} & 50 & 0.11 & 0.017 & 1.16(0.88-1.52) & 0.18(-0.68-0.83)\end{array}$

(B) Regression of $\ln$ (Mandible length) onto $\ln$ (Body length).

\begin{tabular}{lcccccc} 
Species & Sex & $\mathrm{N}$ & $\mathrm{r}^{2}$ & $\mathrm{p}$-value & $\beta(2.5 \%-97.5 \%)$ & $a(2.5 \%-97.5 \%)$ \\
\hline O. audouini & $\mathrm{F}$ & 70 & 0.30 & $<0.001$ & $1.13(0.92-1.38)$ & $1.61(1.35-1.82)$ \\
O. audouini & $\mathrm{M}$ & 70 & 0.29 & $<0.001$ & $1.44(1.18-1.76)$ & $1.07(0.70-1.37)$ \\
O. dejeanii & $\mathrm{F}$ & 43 & 0.37 & $<0.001$ & $1.15(0.90-1.48)$ & $1.33(0.85-1.70)$ \\
O. dejeanii & $\mathrm{M}$ & 50 & 0.23 & $<0.001$ & $0.93(0.73-1.20)$ & $1.57(1.18-1.87)$
\end{tabular}


Table 2.7: Landmarks defined for geometric morphometric analysis of mandibles (also see Figure 2.7).

\begin{tabular}{l} 
Landmark \\
\hline 1 \\
2 \\
3 \\
4 \\
5 \\
6 \\
7 \\
8
\end{tabular}

\section{Definition}

Anterior base of articulating hinge

Cusp of retinacular tooth \#1 (anterior-superior cusp) of molar

Posterior base of first terebral tooth

Apex of first terebral tooth

Anterior base of first terebral tooth/posterior base of second terebral tooth

Apex of second terebral tooth

Anterior base of second terebral tooth/base of incisor

Apex of incisor 
Table 2.8: Singular values (SV) and percent explained for relative warps (RW).

\begin{tabular}{llcr} 
RW\# & SV & $\%$ & Cum \% \\
\hline 1 & 0.59 & $54.71 \%$ & $54.71 \%$ \\
2 & 0.40 & $24.55 \%$ & $79.27 \%$
\end{tabular}


Table 2.9: Results of regression analyses for scaling. Percent variation in shape left unexplained by independent variable $=\%$ unexp, percent variation in shape explained by independent variable $=1$ - $(\%$ unexp $)=\%$ expla.

\begin{tabular}{lcrr} 
MN & sum d $^{2}$ & \% unexp & \% expla \\
\hline af & 0.005008 & $92.42 \%$ & $7.58 \%$ \\
am & 0.001337 & $97.48 \%$ & $2.52 \%$ \\
df & 0.001043 & $96.66 \%$ & $3.34 \%$ \\
dm & 0.001123 & $95.70 \%$ & $4.30 \%$ \\
Mean & $\mathbf{0 . 0 0 2 1 2 8}$ & $\mathbf{9 5 . 5 6 \%}$ & $\mathbf{4 . 4 4 \%}$ \\
& & & \\
MNcs & sum d & $\%$ unexp & $\%$ expla \\
af & 0.004023 & $93.91 \%$ & $6.09 \%$ \\
am & 0.000498 & $99.06 \%$ & $0.94 \%$ \\
df & 0.001985 & $93.64 \%$ & $6.36 \%$ \\
dm & 0.001268 & $95.14 \%$ & $4.86 \%$ \\
Mean & $\mathbf{0 . 0 0 1 9 4 4}$ & $\mathbf{9 5 . 4 4 \%}$ & $\mathbf{4 . 5 6 \%}$ \\
& & & \\
PEL & sum d & $\%$ unexp & $\%$ expla \\
af & 0.005008 & $92.42 \%$ & $7.58 \%$ \\
am & 0.002102 & $96.04 \%$ & $3.96 \%$ \\
df & 0.001605 & $94.86 \%$ & $5.14 \%$ \\
dm & 0.001425 & $94.54 \%$ & $5.46 \%$ \\
Mean & $\mathbf{0 . 0 0 2 5 3 5}$ & $\mathbf{9 4 . 4 6 \%}$ & $\mathbf{5 . 5 4 \%}$
\end{tabular}


Table 2.10: Results of sexual size dimorphism (SSD) for (A) O. audouini and (B) $O$. dejeanii. Pronotum+elytra length (PEL) and mandible length (MN) are reported in millimeters while mandible centroid size (MNcs) is in $\mathrm{mm}^{2}$. Sexual size dimorphism (SSD) was determined by a Welch's t-test and significance based on $\alpha \leq 0.05$ : n.s. $=\alpha>$ $0.05, *=\alpha \leq 0.05, * *=\alpha \leq 0.01, * * *=\alpha \leq 0.001$.

\section{(A) O. audouini}

Female $(\mathrm{n}=34) \quad$ Male $(\mathrm{n}=32)$

Variable Mean \pm SD $($ Min - Max $) \quad$ Mean \pm SD $($ Min - Max $) \quad$ SSD

PEL $\quad 12.90 \pm 0.38(11.50-13.45)^{\mathrm{a}} \quad 12.15 \pm 0.38(11.15-13.05) \quad * *$

MN $\quad 2.73 \pm 0.07(2.56-2.86) \quad 3.11 \pm 0.09 \quad(2.90-3.27) \quad * * *$

MNcs $\quad 3.73 \pm 0.11 \quad(3.51-3.97) \quad 4.16 \pm 0.12 \quad(3.90-4.38) \quad * * *$

a excluding outlier (O. audouini female, ID \#36)

(B) O. dejeanii

\begin{tabular}{lrrrrr} 
& \multicolumn{2}{c}{ Female $(\mathrm{n}=22)$} & \multicolumn{2}{c}{ Male $(\mathrm{n}=22)$} & \\
Variable & Mean \pm SD & $($ Min - Max $)$ & \multicolumn{1}{c}{ Mean \pm SD $($ Min - Max $)$} & SSD \\
\hline PEL & $15.94 \pm 0.49$ & $(15.00-16.80)$ & $14.82 \pm 0.48(13.80-15.40)$ & $* * *$ \\
MN & $4.29 \pm 0.18$ & $(4.01-4.70)$ & $4.27 \pm 0.16$ & $(3.93-4.55)$ & n.s. \\
MNcs & $5.70 \pm 0.23(5.30-6.14)$ & $5.61 \pm 0.19$ & $(5.26-5.96)$ & n.s.
\end{tabular}


Table 2.11: Hotelling-Lawley/Hotelling's $\mathrm{T}^{2}$ test for sexual shape dimorphism.

\begin{tabular}{lcccc} 
Species & Value & $\mathrm{F}$ & d.f. & p-value \\
\hline O. audouini & 38.59 & 39.80 & 32,33 & $<0.0001$ \\
O. dejeanii & 35.06 & 12.05 & 32,11 & $<0.0001$
\end{tabular}


Table 2.12: Summary of proximal and distal terebral teeth angle measurements and curve of the incisor. Angle $\mathrm{P}=$ angle of proximal terebral tooth (angle formed by landmarks 1 , $5,4)$, Angle $\mathrm{D}=$ angle of distal terebral tooth (landmarks \# 1, 7,6), C = coefficient of 2nd degree polynomial to describe the curve of the incisor. See Figure 3.3.

$\begin{array}{lcccc}\text { SPSX } & \text { n } & \text { Angle P } & \text { Angle D } & C \\ \text { af } & 34 & 110.6 \pm 3.2 & 94.6 \pm 7.2 & 3.793 \pm 0.346 \\ \text { am } & 32 & 111.9 \pm 3.2 & 96.9 \pm 5.9 & 2.877 \pm 0.321 \\ \text { df } & 22 & 106.0 \pm 4.8 & 92.2 \pm 3.3 & 3.720 \pm 0.428 \\ \text { dm } & 22 & 102.6 \pm 3.4 & 94.6 \pm 3.7 & 3.297 \pm 0.359\end{array}$


Table 2.13: Results of Tukey HSD multiple comparisons of means $(\alpha=0.05)$ for (A \& B) Terebral tooth angles and (C) Outer curvature of incisor (See Figure 3.3). Comp = categories being compared $(\mathrm{af}=$ female $O$. audouini, $\mathrm{am}=$ male $O$. audouini, $\mathrm{df}=$ female $O$. dejeanii, $\mathrm{dm}=$ male $O$. dejeanii $),$ diff = range of confidence interval $(\mathrm{CI})$, lwr and upr $=$ lower and upper limits of CI. Differences between the compared categories are significant if CI does not include zero. n.s. $=$ not significant $(\mathrm{p}$-value $>0.05), *=\mathrm{p}$-value $\leq 0.05, * *=\mathrm{p}$-value $\leq 0.01, * * *=\mathrm{p}$-value $\leq 0.001$

\begin{tabular}{lrrrr} 
(A) Proximal angle & & & \\
Comparison & diff & \multicolumn{1}{l}{ lwr } & \multicolumn{1}{c}{ upr } & \multicolumn{1}{c}{ p adj } \\
am-af & 1.31 & -1.01 & 3.63 & 0.45 n.s. \\
df-af & -4.59 & -7.16 & -2.01 & $0.00 * * *$ \\
dm-af & -7.95 & -10.53 & -5.37 & $0.00 * * *$ \\
df-am & -5.90 & -8.51 & -3.29 & $0.00 * * *$ \\
dm-am & -9.26 & -11.87 & -6.65 & $0.00 * * *$ \\
dm-df & -3.36 & -6.20 & -0.52 & $0.01 *$
\end{tabular}

(B) Distal angle

Comparison diff

\begin{tabular}{lrrrl}
\hline am-af & 2.35 & -1.21 & 5.92 & 0.32 n.s. \\
df-af & -2.35 & -6.32 & 1.61 & 0.41 n.s. \\
dm-af & -0.01 & -3.97 & 3.96 & 1.00 n.s. \\
df-am & -4.71 & -8.72 & -0.70 & $0.01 * *$ \\
dm-am & -2.36 & -6.37 & 1.65 & 0.42 n.s. \\
dm-df & 2.35 & -2.02 & 6.71 & 0.50 n.s.
\end{tabular}

(C) Outer curvature of incisor ( $\mathrm{C}$ coefficient of 2 nd degree polynomial)

Comparison diff

lwr upr p adj

$\begin{array}{lrrrr}\text { am-af } & -0.92 & -1.15 & -0.69 & 0.00 * * * \\ \text { df-af } & -0.07 & -0.33 & 0.18 & 0.88 \text { n.s. } \\ \text { dm-af } & -0.50 & -0.75 & -0.24 & 0.00 * * * \\ \text { df-am } & 0.84 & 0.58 & 1.10 & 0.00 * * * \\ \text { dm-am } & 0.42 & 0.16 & 0.68 & 0.00 * * * \\ \text { dm-df } & -0.42 & -0.71 & -0.14 & 0.00 * * *\end{array}$


Table 3.1: Ethogram of reproductive behaviors for two species of Omus.

\section{Code Behavior name and description}

Behavioral states

Unmounted (UM):

Individuals are unmounted and not engaging in mating related behaviors.

Male passive (MP):

Male does not engage in any gross/overt movement but remains largely passive while mounted on the female; some minor movement of legs and antennae but mostly these are held out or allowed to drag along the substrate. The female is free to ambulate and engage in behaviors not unlike those while unmounted, e.g. grooming, exploring environment with antennae and mandibles, chewing substrate (possibly for water or ingestible organic matter not resolved on video), etc...

Both still (BS):

The male remains mounted on the female but neither beetle engages in any observable, overt movement for more than 30 -seconds. These quiescent periods may continue for upwards of an hour. There may be a difference between a short-term stillness (pause, no movement for between 30 -seconds to five minutes) and a long-term stillness (quiescence, no movement for $>30$ minutes).

Male thrusting (MT):

Male is mounted and begins an episode of thrusting his genitals. The specific types of thrusts observed are detailed as behavioral events. An episode of MT was defined as any identifiable series of male thrusts separated by any other series of thrusting by at least five minutes. See text for details on species differences in thrusting behaviors.

Behavioral events

Mounting position attempt (MPA):

Male attempts to mount another beetle. This involves rapidly attempting to reach a mounted position and proper orientation using adhesive tarsal pads on forelegs, as well as use of mandibles. Female often, but not always, responds to MPA with PCS.

Pre-copulatory struggle (PCS):

Upon male's attempt to mount and clasp, female struggles by rolling and thrashing. In $n$ observed matings, PCS was not seen to regularly undermine a male's attempt to mount. Post-copulatory attempts to remount a female were occasionally undermined by PCS but more often the female was able to simply. Run away from the male upon initial contact and before the male MPA. 
(Table 3.1 cont.)

Antennal stroking/drumming (ASD):

While mounted, the male uses his antennae to stroke/drum the antennae and head of female.

Intromission (INT):

Male extends and inserts copulatory organs. Intromission usually occurs within a few seconds of achieving a stable position on female (male mandibles firmly grasping female).

Male leg kicking/twitching (MLK):

While mounted, the male kicks/twitches his legs. Omus audouini were seen to kick with their back and middle pairs of legs and it was uncertain whether they were contacting any part of the female. O. dejeanii were observed to use their forelegs to stroke and drum against the thorax and leg of the mounted female.

Male thrust, type $n(\mathrm{MT} n)$ :

While mounted, male bends at neck and waist joints to move abdomen and inserted intromittent organ. Male intromittant organ was never seen to be completely withdrawn at any time during thrusting or throughout the normal course of amplexus.

\section{For O. audouini}

MT1: Slow direct insertion and slow withdrawal of male organ; there may be a difference in depth of insertion that was not evident on the video. MT2: Slow insertion while flexing abdomen to the left, presumably causing inserted male organ to flex to the right within the female genital tract.

MT3: Slow direct insertion and relatively quick withdrawal of male organ. For $O$. dejeanii

MT1: A brief series of about a dozen direct, rapid insertions and withdrawals, each taking about 1 second to complete.

MT2: When already deeply inserted, the male then performs a series of half a dozen or less short, direct thrusts

MT3: A slow, direct insertion and withdrawal, the entire cycle taking about 30 seconds to complete; often performed as a single motion or, if in series, with pauses in between.

MT4: Full insertion with an abdominal flexion or roll to the left side, performed singly or in series of about half a dozen; similar to male thrust type 2 for $O$. audouini. 
(Table 3.1 cont.)

Syn-copulatory struggle (SCS):

While mounted and in cotius with male, the female engages in struggle behavior. May range from minor shaking and twitching lasting a couple seconds to full rolling and thrashing. Usually preceding male thrusting or genital withdrawal and dismount by male. Often proceeded and suppressed by antennal stroking by male. 
Table 3.2: Duration of male thrusting episodes during amplexus for (A) O. audouini and (B) O. dejeanii. PAIR = the specific pair of beetles. "OB $\mathrm{n}$ " = the observation number where " 1 " indicates a thrusting even between an hour post-initial contact and approximately midway through the mating duration, "2" indicates an observation at approximately halfway through the mating duration and " 3 " indicates an observation after the midway observation and termination of mating. "DUR" = duration of the thrusting episode in minutes. "nTH" = the number of thrusts observed for that episode (for O. audouini only, see text for details), "SPM" = number of strokes per minutes for that observation (i.e. $=$ nTH/DUR).

\begin{tabular}{|c|c|c|c|c|}
\hline PAIR & $\mathrm{B} n$ & DUR & nTH & SPM \\
\hline AF62/AM91 & 1 & 105.57 & 154 & 1.46 \\
\hline AF62/AM91 & 2 & 37.30 & 37 & 0.99 \\
\hline AF62/AM91 & 3 & 58.73 & 81 & 1.38 \\
\hline Mean \pm SD & & $67.2 \pm 35.9$ & $90.7 \pm 59.1$ & $1.3 \pm 0.3$ \\
\hline AF66/AM84 & 1 & 103.42 & 158 & 1.53 \\
\hline AF66/AM84 & 2 & 90.03 & 149 & 1.65 \\
\hline AF66/AM84 & 3 & 84.73 & 129 & 1.52 \\
\hline Mean \pm SD & & $92.7 \pm 9.6$ & $145.3 \pm 14.8$ & $1.6 \pm 0.1$ \\
\hline AF67/AM92 & 1 & 21.88 & 21 & 0.96 \\
\hline AF67/AM92 & 2 & 25.47 & 47 & 1.85 \\
\hline AF67/AM92 & 3 & 70.57 & 109 & 1.54 \\
\hline Mean \pm SD & & $39.3 \pm 27.1$ & $59.0 \pm 45.2$ & $1.5 \pm 0.5$ \\
\hline AF77/AM82 & 1 & 108.80 & 198 & 1.82 \\
\hline AF77/AM82 & 2 & 86.95 & 138 & 1.59 \\
\hline AF77/AM82 & 3 & 74.47 & 145 & 1.95 \\
\hline Mean \pm SD & & $90.1 \pm 17.4$ & $160.3 \pm 32.8$ & $1.8 \pm 0.2$ \\
\hline Total mean \pm & SD & $72.3 \pm 30.5$ & $113.8 \pm 55.4$ & $1.5 \pm 0.3$ \\
\hline
\end{tabular}


Table 3.2 (cont.)

(B) Omus dejeanii

\begin{tabular}{lcc} 
PAIR & OB n & DUR \\
DF33/DM55 & 1 & 102.10 \\
DF33/DM55 & 2 & 124.80 \\
DF33/DM55 & 3 & 104.30 \\
\hline Mean \pm SD & & $110.4 \pm 12.5$ \\
& & \\
DF57/DM95 & 1 & 106.65 \\
DF57/DM95 & 2 & 78.48 \\
DF57/DM95 & 3 & 75.60 \\
\hline Mean \pm SD & & $86.9 \pm 17.2$ \\
& & 101.77 \\
DF59/DM24 & 1 & 80.67 \\
DF59/DM24 & 2 & 70.05 \\
DF59/DM24 & 3 & $84.2 \pm 16.2$ \\
\hline Mean \pm SD & & 197.23 \\
DF95/DM52 & 1 & 171.43 \\
DF95/DM52 & 2 & 113.17 \\
DF95/DM52 & 3 & $160.6 \pm 43.1$ \\
\hline Mean \pm SD & & $\mathbf{1 1 0 . 5} \pm \mathbf{3 8 . 6}$ \\
Total mean \pm SD &
\end{tabular}


Table 3.3: Features speculated to confer defensive advantage for Omus. Type: $\mathrm{A}=$ anatomical, $\mathrm{B}=$ behavioral.

$\begin{array}{lcll}\text { Defense } & \text { Type Defensive advantage } & \text { Alternate advantage } \\ \text { Cuticle color } & \text { A } & \text { Crypsis } & \text { Thermoregulation } \\ \text { Cuticle thickness } & \text { A } & \text { Armor } & \text { Water retention, durability } \\ \text { Gross morphology } & \text { A } & \text { Less purchase } & \text { Economy of development } \\ \text { Non-gregarious } & \text { B } & \text { Concealment, low-profile } & \text { Absence of social complexity } \\ \text { Immobility } & \text { B } & \text { Concealment } & \text { Energetic economy } \\ \text { Hiding in duff } & \text { B Avoidance, concealment } & \text { Thermoregulation/insulation } \\ \text { Running } & \text { B Evasion, escape } & \begin{array}{l}\text { Hunting behavior co-opted to } \\ \text { defense }\end{array} \\ \text { Biting } & \text { B } \text { Discouragement } & \begin{array}{l}\text { Hunting behavior co-opted to } \\ \text { defense }\end{array} \\ \text { Emesis } & \text { B } & \text { Pain inducement (enzymes) } & \text { Stress }\end{array}$


Table 4.1: Body length results of (A) two species of Omus and (B) tiger beetle species used for evaluating trends of sexual size dimorphism. Values are in $\mathrm{mm}$.

(A) Body length results of $O$. audouini and $O$. dejeanii.

Species, sex $\quad \mathrm{n} \quad$ Mean body length \pm SD (Min-Max)

O. audouini, female $70 \quad 16.20 \pm 0.69(14.0-17.5)$

O. audouini, male $70 \quad 15.05 \pm 0.65(13.5-16.6)$

O. dejeanii, female $43 \quad 20.55 \pm 0.70(19.0-22.0)$

O. dejeanii, male $\quad 50 \quad 18.93 \pm 0.72(17.5-21.5)$

(B) Body lengths of cicindelines used for determining among species sexual size dimorphism allometry. Sources of body lengths indicated by superscripts. $F=$ mean female body length in $\mathrm{mm}, \mathrm{M}=$ mean male body length in $\mathrm{mm}$. SDI = Size dimorphism index (Lovich \& Gibbons 1992).

\begin{tabular}{|c|c|c|c|c|c|}
\hline Species (abbreviation) & $\mathrm{F}$ & M & $\ln \mathrm{F}$ & $\ln \mathrm{M}$ & SDI \\
\hline Cicindela nigrocoerulea $(\mathrm{CINI})^{\mathrm{a}}$ & 12.83 & 12.14 & 2.552 & 2.497 & 0.057 \\
\hline Cicindela sexguttata (CISE) $^{\mathrm{a}}$ & 13.73 & 12.54 & 2.620 & 2.529 & 0.095 \\
\hline Cicindela abdominalis (CIAB) ${ }^{\mathrm{a}}$ & 9.79 & 8.79 & 2.281 & 2.174 & 0.114 \\
\hline 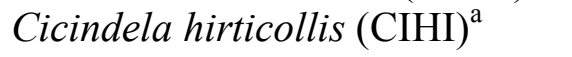 & 13.23 & 11.79 & 2.583 & 2.467 & 0.122 \\
\hline Cicindela cuprascens $(\mathrm{CICU})^{\mathrm{a}}$ & 12.21 & 11.42 & 2.502 & 2.435 & 0.069 \\
\hline Cicindela marutha (CIMA) ${ }^{\mathrm{b}}$ & 12.4 & 11.5 & 2.518 & 2.442 & 0.078 \\
\hline Omus audouini (OMAU) ${ }^{\mathrm{c}}$ & 16.2 & 15.1 & 2.785 & 2.711 & 0.076 \\
\hline Omus dejeanii (OMDE) ${ }^{\mathrm{c}}$ & 20.6 & 18.9 & 3.023 & 2.941 & 0.086 \\
\hline Mean SDI \pm SD & & & & & 0.087 \\
\hline
\end{tabular}

${ }^{\mathrm{a}}$ Kritsky and Simon, 1995

${ }^{\mathrm{b}}$ Kraus and Lederhouse, 1983

${ }^{\mathrm{c}}$ This study 
Table 4.2: Results and summary statistics for (A) O. audouini and (B) O. dejeanii. Pair is the specific pair of beetles with unique id code, date is date of pairing, day is number of days from 01 June 2011 as an arbitrary measure of seasonality, time is whether the mating was initiated at 08:00 (am) or at 18:00 (pm), durh is duration of mating in hours, bl $x$ is body length in millimeters, $\mathrm{mn} x$ is mandible length in millimeters and ww $x$ is waist width in millimeters where $x=f$ for female and $m$ for male.

(A) O. audouini

\begin{tabular}{|c|c|c|c|c|c|c|c|c|c|c|}
\hline pair & date & day & time & durh & blf & blm & $\mathrm{mnf}$ & $\mathrm{mnm}$ & wwf & wwm \\
\hline af77/am82 & 20-Jun-11 & 20 & $\mathrm{am}$ & 12.0 & 16.1 & 15.5 & 3.10 & 3.20 & 3.25 & 3.00 \\
\hline af78/am94 & 26-Jun-11 & 26 & $\mathrm{am}$ & 11.7 & 17.8 & 15.2 & 3.10 & 3.20 & 3.25 & 2.95 \\
\hline f62/am91 & 3-Jul-11 & 33 & $\mathrm{am}$ & 11.4 & 16.7 & 15.0 & 3.20 & 3.15 & 3.10 & 3.00 \\
\hline f73/am79 & 9-Jul-11 & 39 & $\mathrm{am}$ & 9.6 & 17.4 & 16.0 & 3.00 & 3.25 & 3.25 & 3.10 \\
\hline 774/am90 & 16-Jul-11 & 46 & $\mathrm{am}$ & 7.8 & 16.1 & 14.8 & 2.85 & 3.15 & 3.10 & 2.75 \\
\hline f67/am92 & 18-Jun-11 & 18 & $\mathrm{pm}$ & 8.9 & 15.5 & 14.7 & 2.80 & 3.10 & 3.10 & 2.85 \\
\hline af70/am88 & 24-Jun-11 & 24 & $\mathrm{pm}$ & 13.5 & 17.3 & 15.4 & 3.15 & 3.15 & 3.15 & 3.00 \\
\hline $66 / a m 84$ & 1-Jul-11 & 31 & $\mathrm{pm}$ & 10.5 & 16.4 & 14.4 & 3.00 & 3.15 & 3.15 & 2.90 \\
\hline f69/am86 & 7-Jul-11 & 37 & $\mathrm{pm}$ & 18.3 & 16.7 & 14.9 & 2.90 & 3.20 & 3.15 & 2.90 \\
\hline af71/am83 & 14-Jul-11 & 44 & $\mathrm{pm}$ & 10.1 & 18.1 & 15.0 & 3.10 & 3.30 & 3.30 & 2.95 \\
\hline Mean $^{\mathrm{b}}$ & & & & 11.4 & 16.8 & 15.1 & 3.02 & 3.19 & 3.18 & 2.94 \\
\hline $\mathrm{SD}^{\mathrm{b}}$ & & & & 2.9 & 0.8 & 0.5 & 0.13 & 0.06 & 0.08 & 0.10 \\
\hline Hean $^{\mathrm{c}}$ & & & & 10.6 & 16.8 & 15.1 & 3.03 & 3.18 & 3.18 & 2.94 \\
\hline $\mathrm{SD}^{\mathrm{c}}$ & & & & 1.7 & 0.9 & 0.5 & 0.14 & 0.06 & 0.08 & 0.10 \\
\hline
\end{tabular}

${ }^{a}$ This pair had a mating duration greater than two standard deviations: Mean $+\mathrm{SD}(2)=$ $11.4+2.9(2)=11.4+5.8=17.2$ hours. As such they were regarded as not representative of typical mating behavior regarded as an outlier and removed from the dataset for statistical tests. They were not outliers on any other variable.

${ }^{b}$ For dataset including pair af69/am86.

${ }^{\mathrm{c}}$ For dataset excluding outlier pair af69/am86.

(B) O. dejeanii

\begin{tabular}{lrccccccccc} 
pair & \multicolumn{1}{c}{ date } & day & time & durh & blf & blm & mnf & mnm & wwf & wwm \\
df33/dm55 & 23-Jun-11 & 23 & am & 26.0 & 22.6 & 19.3 & 4.80 & 4.40 & 4.05 & 3.60 \\
df59/dm24 & 29-Jun-11 & 29 & am & 34.3 & 22.1 & 19.7 & 4.60 & 4.35 & 4.00 & 3.65 \\
df56/dm98 & 6-Jul-11 & 36 & am & 20.8 & 21.3 & 20.1 & 4.50 & 4.55 & 3.95 & 3.65 \\
df95/dm52 & 12-Jul-11 & 42 & am & 39.7 & 21.0 & 19.1 & 4.70 & 4.50 & 3.90 & 3.50 \\
df45/dm150 & 19-Jul-11 & 49 & am & 25.1 & 20.3 & 18.0 & 4.40 & 4.35 & 3.75 & 3.45 \\
df101/dm96 & 21-Jun-11 & 21 & pm & 28.1 & 22.0 & 19.5 & 4.80 & 4.40 & 4.05 & 3.50 \\
df97/dm100 & 27-Jun-11 & 27 & pm & 26.4 & 21.7 & 18.6 & 4.40 & 4.40 & 3.90 & 3.55 \\
df57/dm95 & 4-Jul-11 & 34 & pm & 27.8 & 21.0 & 19.1 & 4.40 & 4.40 & 4.05 & 3.65 \\
df44/dm29 & 10-Jul-11 & 40 & pm & 30.9 & 21.5 & 19.1 & 4.60 & 4.40 & 3.95 & 3.70 \\
df99/dm97 & 16-Jul-11 & 46 & pm & 35.4 & 20.7 & 20.1 & 4.45 & 4.35 & 3.90 & 3.65 \\
\hline Mean & & & & 29.4 & 21.4 & 19.3 & 4.57 & 4.41 & 3.95 & 3.59 \\
SD & & & & 5.6 & 0.7 & 0.7 & 0.16 & 0.07 & 0.09 & 0.08
\end{tabular}


Table 4.3: Mean mating duration for $O$. audouini and $O$. dejeanii for time of initiation experiments (in minutes and hours).

\begin{tabular}{|c|c|c|c|}
\hline Species & $\mathrm{n}$ & Mean $\pm \mathrm{SD}(\min )$ & Mean \pm SD (hours) \\
\hline O. audouini & 9 & $637 \pm 105$ & $10.6 \pm 1.7$ \\
\hline O. dejeanii & 10 & $1766 \pm 338$ & $29.4 \pm 5.6$ \\
\hline
\end{tabular}


Table 4.4: Regression model details for mating duration for O. audouini. (A) Multiple regression model, (B) simple regression model of only female mandible length as the predictor variable.

(A) Best-fit multiple regression model of mating duration for O. audouini. Formula: $d u r h=13.23+11.76 f m n+19.70 f w w-31.72 m m n$, adjusted $\mathrm{R}^{2}=0.87$, F-value $_{3,5}=$ 19.37, p-value $=0.004)$.

\begin{tabular}{lrrrr} 
& Estimate & \multicolumn{1}{l}{ SE } & t-value & $\operatorname{Pr}(>|\mathrm{t}|)$ \\
\hline intercept & 13.23 & 11.93 & 1.11 & 0.32 \\
fmn & 11.76 & 1.77 & 6.66 & $<0.00$ \\
fww & 19.70 & 6.50 & 3.03 & 0.03 \\
mmn & -31.72 & 8.38 & -3.78 & 0.01
\end{tabular}

(B) Simple regression model of mating duration on female mandible length for $O$. audouini. Formula: durh $=-21.85+10.70 \mathrm{fmn}$, multiple $\mathrm{R}^{2}=0.69$, F-value ${ }_{1,7}=15.29$, $\mathrm{p}$ value $=0.006$ ).

\begin{tabular}{lrrrc} 
& Estimate & SE & t-value & $\operatorname{Pr}(>|t|)$ \\
\hline intercept & -21.85 & 8.31 & -2.63 & 0.03 \\
fmn & 10.70 & 2.74 & 3.91 & $<0.00$
\end{tabular}


Table 4.5: Analysis of variance table for best-fit multiple regression model of mating duration in hours for $O$. audouini.

\begin{tabular}{lcrrrc} 
Variable & d.f. & \multicolumn{1}{c}{ SS } & \multicolumn{1}{c}{ MSS } & F-value & $\operatorname{Pr}(>\mathrm{F})$ \\
\hline fmn & 1 & 16.61 & 16.61 & 43.29 & $<0.00$ \\
fww & 1 & 0.19 & 0.19 & 0.51 & 0.51 \\
mmn & 1 & 5.49 & 5.49 & 14.32 & 0.01 \\
Residuals & 5 & 1.92 & 0.38 & - & -
\end{tabular}


Table 4.6: Pearson correlation coefficients between individual predictor variables and mating duration in hours for O. audouini and O. dejeanii.

\begin{tabular}{llllllll} 
Species & day & $\mathrm{fbl}$ & $\mathrm{mbl}$ & $\mathrm{fmn}$ & $\mathrm{mmn}$ & fww & mww \\
\hline O. audouini & -0.52 & 0.37 & 0.33 & 0.83 & 0.04 & 0.23 & 0.60 \\
O. dejeanii & 0.27 & 0.12 & 0.16 & 0.23 & 0.18 & 0.00 & 0.00
\end{tabular}


Table 4.7: Attempt to determine whether mating duration can be presented as a function of mass as inferred by the $3 / 4$ scaling law. (A) O. audouini, (B) O. dejeanii. durh $=$ duration (in hours), $\mathrm{fbl}=$ female body length, $\mathrm{mbl}=$ male body length. All results were non-significant.

(A) O. audouini.

\begin{tabular}{lcccc} 
Model & Slope estimate & p-value & $\mathrm{R}^{2}$ & F-value $($ d.f. $=1,7)$ \\
\hline durh $\sim$ fbl & 0.73 & 0.33 & 0.13 & 1.08 \\
durh $\sim$ fbl $^{3}$ & $<0.01$ & 0.36 & 0.12 & 0.97 \\
durh $\sim \mathrm{mbl}^{3}$ & 1.20 & 0.39 & 0.11 & 0.86 \\
durh $\sim$ mbl $^{3}$ & $<0.01$ & 0.41 & 0.10 & 0.78
\end{tabular}

(B) O. dejeanii

\begin{tabular}{lcccc} 
Model & Slope estimate & p-value & $\mathrm{R}^{2}$ & F-value $($ d.f. $=1,8)$ \\
\hline durh $\sim \mathrm{fbl}$ & -0.97 & 0.74 & 0.02 & 0.12 \\
durh $\sim \mathrm{fbl}^{3}$ & $<-0.01$ & 0.73 & 0.02 & 0.13 \\
durh $\sim \mathrm{mbl}^{3}$ & 1.41 & 0.66 & 0.03 & 0.21 \\
durh $\sim \mathrm{mbl}^{3}$ & $<0.01$ & 0.68 & 0.02 & 0.18
\end{tabular}


Table 4.8: Details of pairings for 2012 food deprivation experiments. Species ("SP"): A $=O$. audouini, $\mathrm{D}=O$. dejeanii. Experiment type (TYPE): see text for explanations. Date and time of initiation of experiment/video file (DATE-TIME) = year month day-hour minute second. Mating duration in minutes $(\mathrm{MDm})=$ mating episode of maximum duration (in minutes). Mating duration in hours $(\mathrm{MDh})=$ mating episode of maximum duration (in hours).

$\begin{array}{lcccr}\text { SP } & \text { TYPE } & \text { DATE-TIME } & \text { MDm } & \text { MDh } \\ \text { A } & \text { F01/M01 } & 20120806-143111 & 597.4 & 10.0 \\ \text { A } & \text { F01/M01 } & 20120806-143111 & 712.4 & 11.9 \\ \text { A } & \text { F01/M01 } & 20120723-151431 & 751.4 & 12.5 \\ \text { A } & \text { F01/M24 } & 20120712-154133 & 526.9 & 8.8 \\ \text { A } & \text { F01/M24 } & 20120727-154707 & 748.6 & 12.5 \\ \text { A } & \text { F01/M48 } & 20120801-093305 & 502.5 & 8.4 \\ \text { A } & \text { F01/M48 } & 20120711-153011 & 510.3 & 8.5 \\ \text { A } & \text { F24/M01 } & 20120801-093305 & 495.6 & 8.3 \\ \text { A } & \text { F24/M01 } & 20120712-154133 & 941.4 & 15.7 \\ \text { A } & \text { F24/M24 } & 20120802-120647 & 305.4 & 5.1 \\ \text { A } & \text { F24/M24 } & 20120802-120647 & 350.2 & 5.8 \\ \text { A } & \text { F24/M48 } & 20120725-140810 & 494.6 & 8.2 \\ \text { A } & \text { F24/M48 } & 20120725-140810 & 712.0 & 11.9 \\ \text { A } & \text { F48/M01 } & 20120711-153011 & 637.7 & 10.6 \\ \text { A } & \text { F48/M01 } & 20120727-154707 & 770.3 & 12.8 \\ \text { A } & \text { F48/M24 } & 20120719-124128 & 469.0 & 7.8 \\ \text { A } & \text { F48/M24 } & 20120719-124128 & 706.0 & 11.8 \\ \text { A } & \text { F48/M48 } & 20120722-144858 & 628.2 & 10.5 \\ \text { A } & \text { F48/M48 } & 20120722-144858 & 727.8 & 12.1 \\ \text { D } & \text { F01/M01 } & 20120730-110139 & 1658.0 & 27.6 \\ \text { D } & \text { F01/M24 } & 20120709-140355 & 2409.5 & 40.2 \\ \text { D } & \text { F01/M48 } & 20120716-141416 & 1725.6 & 28.8 \\ \text { D } & \text { F24/M01 } & 20120709-140355 & 2153.6 & 35.9 \\ \text { D } & \text { F24/M24 } & 20120804-140413 & 1044.9 & 17.4 \\ \text { D } & \text { F48/M01 } & 20120716-141416 & 1693.1 & 28.2 \\ \text { D } & \text { F48/M48 } & 20120730-110139 & 1301.8 & 21.7\end{array}$


Table 4.9: Best-fit multiple regression model details for mating duration for food deprivation experiment. (A) O. audouini, (B) O. dejeanii.

(A) Omus audouini model: duration (in hours) $=21.61-0.19$ (female age -0.10 (male captivity), adjusted $\mathrm{R}^{2}=0.185, \mathrm{~F}$-value $2,15=2.93$, $\mathrm{p}$-value $\left.=0.085\right)$.

\begin{tabular}{lcccc} 
& Estimate & SE & t-value & $\operatorname{Pr}(>|t|)$ \\
\hline intercept & 21.62 & 4.77 & 4.53 & $<0.001$ \\
female age & -0.19 & 0.08 & -2.38 & 0.031 \\
male captivity & -0.10 & 0.07 & -1.45 & $0.168^{\mathrm{a}}$
\end{tabular}

${ }^{a}$ A model of mating duration as a function of female age, excluding male captivity, had a $\mathrm{p}$-value $=0.08$.

(B) Omus dejeanii model: duration (in hours) $=-370.09+25.75$ (female elytra) -9.81 (male elytra), adjusted $\mathrm{R}^{2}=0.679, \mathrm{~F}_{\text {-value }}, 4=7.33$, $\mathrm{p}$-value $=0.046$ ).

\begin{tabular}{lrrrr} 
& Estimate & \multicolumn{1}{c}{$\mathrm{SE}$} & $\mathrm{t}$-value & $\operatorname{Pr}(>|\mathrm{t}|)$ \\
intercept & -370.09 & 105.36 & -3.51 & 0.025 \\
female elytra & 25.75 & 8.06 & 3.19 & 0.033 \\
male elytra & 9.81 & 4.29 & 2.29 & 0.084
\end{tabular}


Table 4.10: Details of pairings for 2012 operational sex ratio experiments. Species (SP): $\mathrm{A}=$ O. audouini, $\mathrm{D}=O$. dejeanii. Experiment type (TYPE): see text for explanations. Date and time of initiation of experiment/video file $($ DATE-TIME $)=$ date $($ year month day) - time (hour minute second). Mating duration in minutes $(\mathrm{MDm})=$ mating episode of maximum duration (in minutes). Mating duration in hours (MDh) = mating episode of maximum duration (in hours).

\begin{tabular}{lcccrr} 
SP & EXP & TYPE & DATE-TIME & MDm & MDh \\
\hline A & osr & FFM & $20120726-151501$ & 442.8 & 7.4 \\
A & osr & FFM & $20120708-120053$ & 686.5 & 11.4 \\
A & osr & FMM & $20120726-151501$ & 384.9 & 6.4 \\
A & osr & FMM & $20120803-135354$ & 486.3 & 8.1 \\
A & osr & FMM & $20120708-120053$ & 1873.2 & 31.2 \\
A & osr & FMX & $20120803-135354$ & 1619.3 & 27.0 \\
D & osr & FFM & $20120714-141803$ & 1231.4 & 20.5 \\
D & osr & FMM & $20120714-141803$ & 1679.1 & 28.0 \\
D & osr & FMX & $20120720-143755$ & 707.1 & 11.8 \\
D & osr & FMX & $20120720-143755$ & 1612.9 & 26.9
\end{tabular}


Table 4.11: Results of effects of operational sex ratio on mating duration. (A) $O$. audouini, (B) Omus dejeanii, (C) Results of ANOVA testing whether any of the operational sex ratio treatments were significantly different from matings observed under equal sex ratios. $\mathrm{TX}=$ treatment type: $\mathrm{FM}=$ one female, one male; $\mathrm{FFM}=$ Two females, one male (female-biased sex ratio); FMM = One female, two males (male-biased sex ratio); FMX = One female, one male and one sympatric non-Omus carabid of approximately the same size (Scaphinotus marginatus for $O$. audouini and $S$. angusticollis for $O$. dejeanii).

\section{(A) Omus audouini}

\begin{tabular}{lrr} 
TX & N & Mean \pm SD \\
\hline FM & 18 & $611 \pm 164$ \\
FFM & 2 & $652 \pm 78$ \\
FMM & 3 & $505 \pm 160$ \\
FMX & 1 & 486
\end{tabular}

(B) Omus dejeanii

\begin{tabular}{lll} 
TX & N & Mean \pm SD \\
\hline FM & 7 & $1712 \pm 465$ \\
FFM & 1 & 1679 \\
FMM & 2 & $1746 \pm 180$ \\
FMX & 2 & $1422 \pm 270$
\end{tabular}

(C) Results of ANOVA testing for differences among treatments

Species d.f.

O. audouini

1,2

SS
MS

O. dejeanii

$1,1034766 \quad 34766$ F-stat P-value

$\begin{array}{ll}0.65 & 0.43\end{array}$

$\begin{array}{ll}0.23 & 0.64\end{array}$


Table 4.12: Individuals collected over three years, (A) catch totals and (B) sex ratios. AF $=O$. audouini, female; $\mathrm{AM}=O$. audouini, male; $\mathrm{DF}=O$. dejeanii, female; $\mathrm{DM}=O$. dejeanii, male.

(A) Year catch totals.

\begin{tabular}{lcccc} 
Year & AF & AM & DF & DM \\
\hline 2010 & 47 & 55 & 26 & 37 \\
2011 & 45 & 38 & 12 & 10 \\
2012 & 65 & 48 & 11 & 10 \\
\hline TOTAL & 157 & 141 & 49 & 57
\end{tabular}

(B) Year sex-ratios

\begin{tabular}{lcc} 
Year & $\mathrm{A}: \mathrm{F} / \mathrm{M}$ & $\mathrm{D}: \mathrm{F} / \mathrm{M}$ \\
\hline 2010 & 0.85 & 0.70 \\
2011 & 1.18 & 1.20 \\
2012 & 1.35 & 1.10 \\
\hline Mean & 1.13 & 1.00
\end{tabular}


Table 4.13: $\chi^{2}$-test for equal proportions of sexes over three years.

$\begin{array}{lccccc}\text { Species } & \text { Mean F } & \text { Mean M } & \chi^{2} & \text { d.f. } & \text { p-value } \\ \text { O. audouini } & 52.33 & 47.00 & 0.29 & 2 & 0.59 \\ \text { O. dejeanii } & 16.33 & 19.00 & 0.20 & 2 & 0.65\end{array}$


$\underline{\text { Figures }}$

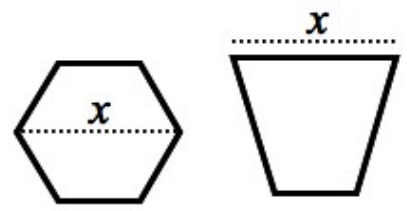

Figure 1.1: Two shapes where the greatest width is the same but points used to define greatest width are not homologous. 


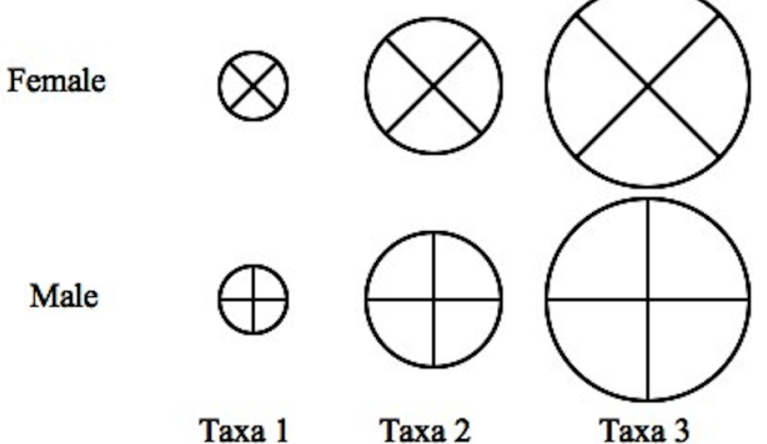

(A) Rensche's rule, SSD isometry: Either there is no sexual size dimorphism or the degree of SSD is constant among taxa independent of body size.

Female
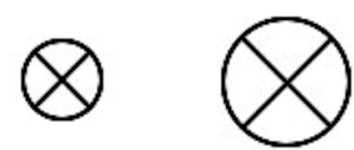

Male
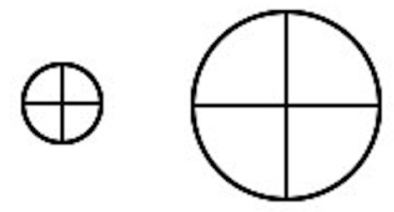

Taxa 1

Taxa 2

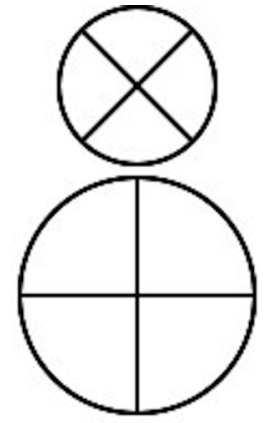

Taxa 3

(B) Rensche's rule, SSD, male biased: males become proportionally larger than females as general body size increases among related taxa.

Female
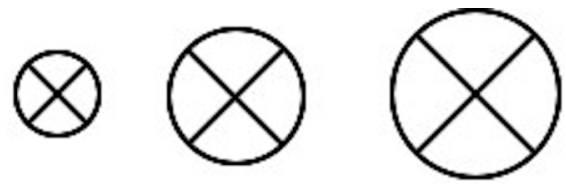

Male

$\oplus$

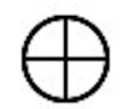

\section{Taxa 1 Taxa 2 Taxa 3}

(C) Rensche's rule: SSD, female biased: males become proportionally smaller than females as general body size decreases among related taxa.

Figure 1.2: Rensche's rule of among species trends in sexual size dimorphism, paraphrased as male body size shows greater variation than female body size (Blanckenhorn et al 2007). (A) SSD, isometry, (B) SSD, male-biased, (C) SSD, femalebiased. 


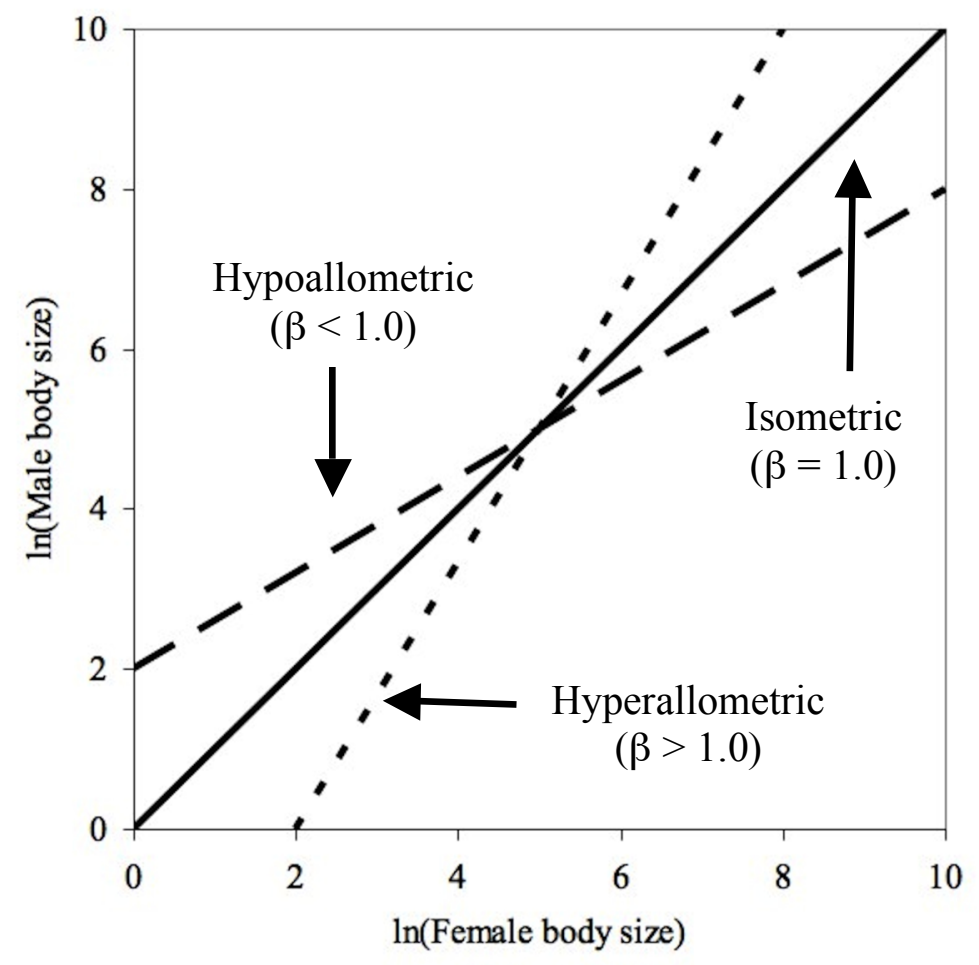

Figure 1.3: Hypothetical allometric scaling of sexual size dimorphism. Solid line represents isometry $(\beta=1.0)$, proportional size differences between females and males are constant across body sizes for interspecific size classes. Dashed line represents hypoallometry $(\beta<1.0)$, where there is greater variation seen in female body size than male body size across interspecific size classes. Dotted line represents hyperallometry ( $\beta$ $>1.0$ ), where there is greater variation seen in female body size than male body size across interspecific size classes. Figure derived from Fairbairn 1997. 


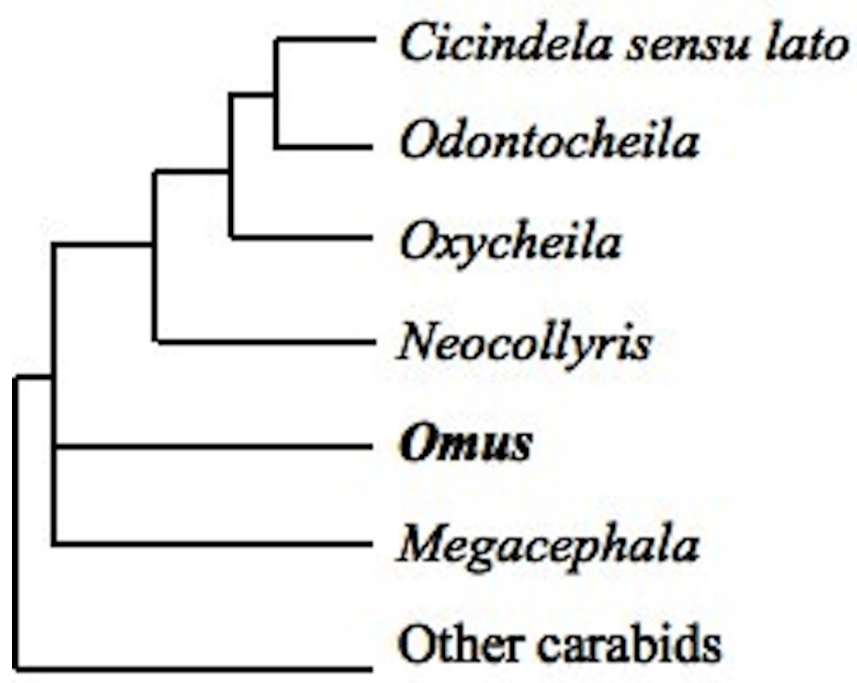

Figure 1.4: Cicindeline phylogeny (from Maddison et al. 1999 \& Vogler et al. 2005) 


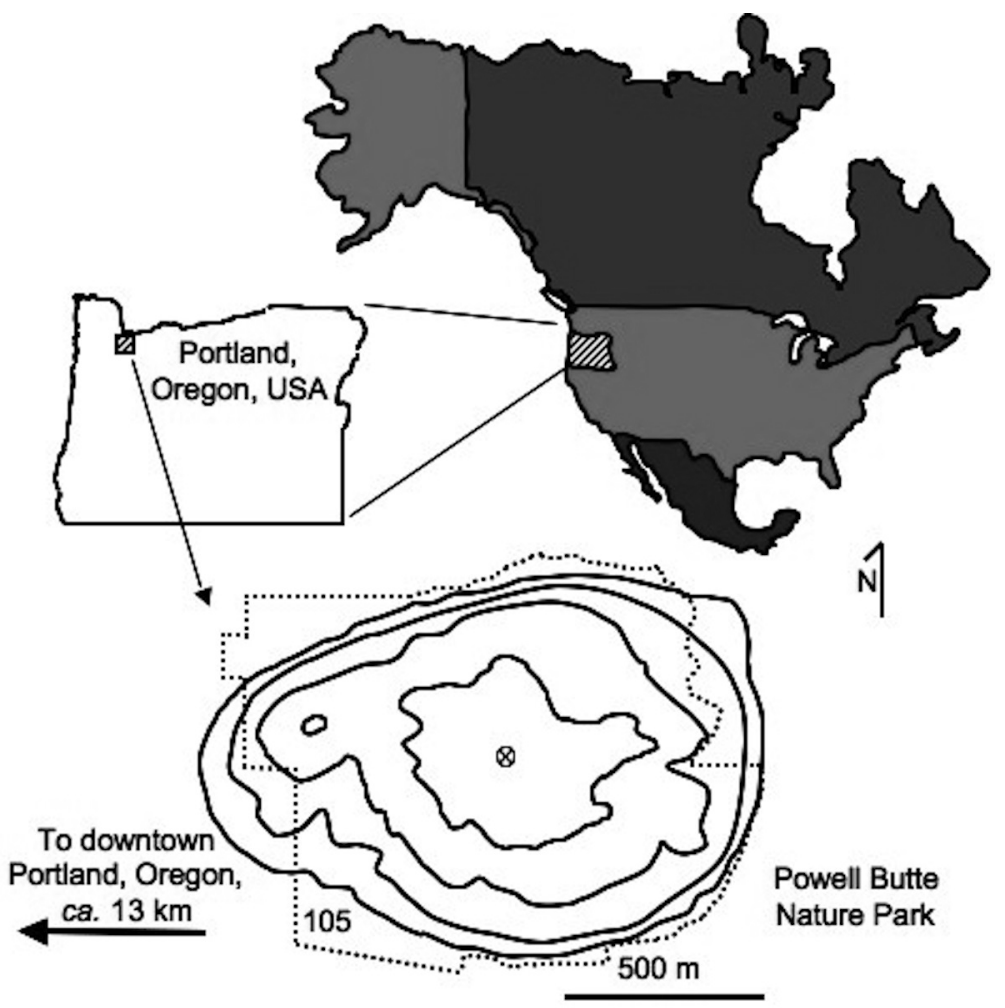

Figure 1.5 Powell Butte Nature Park, Portland, Oregon. 45 $29^{\prime} 14.5^{\prime \prime} \mathrm{N}$ $122^{\circ} 30^{\prime} 06.5^{\prime \prime} \mathrm{W}$. Contours at ca. 15 meters, summit (marked as $\left.\otimes\right)$ ca. $190 \mathrm{~m}$. 


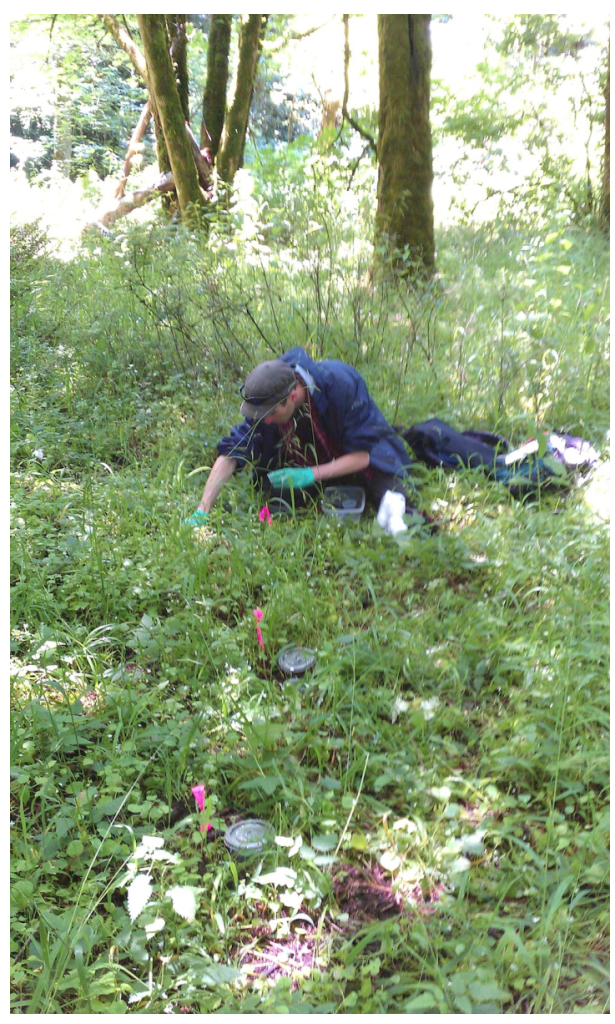

Figure 1.6 The author (R.K.R.) at Powell Butte, checking pitfall traps, 27 June 2012. 

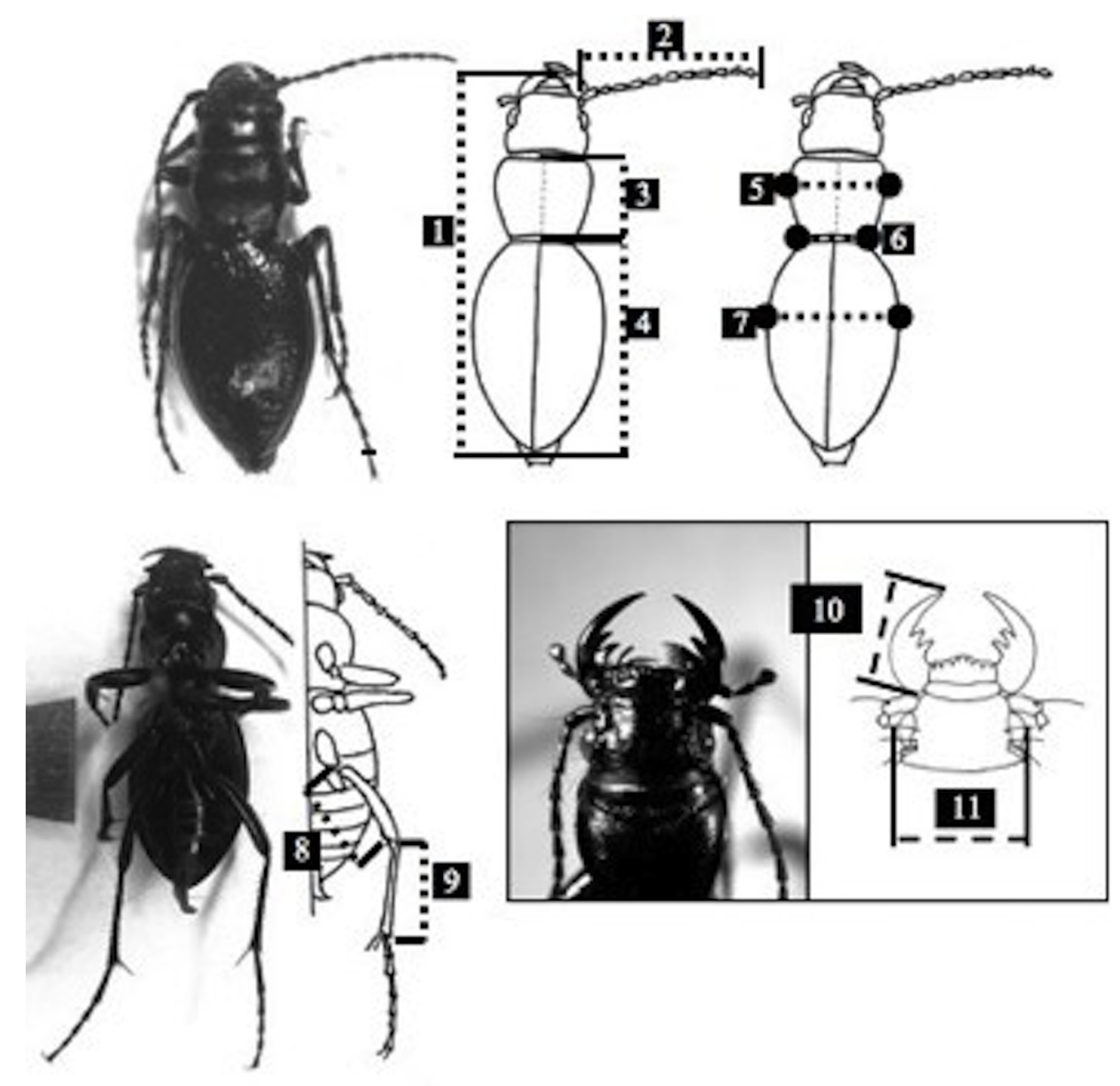

Figure 2.1: Morphometric features measured for gross anatomical analysis. Eleven linear measurements: 1. Body Length, 2. Antennal length (right), 3. Pronotum length, 4. Elytra length, 5. Pronotum width, 6. Waist width, 7. Elytra width, 8. Femur length, 9. Tibia length, 10. Mandible length, 11. Head width (inc. eyes). See Table 2.4 for definitions. 


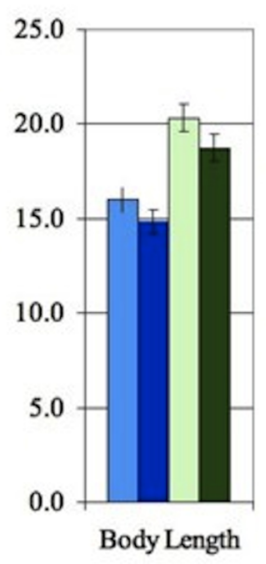

Values are reported in $\mathrm{mm}$

* $=$ n.s. $(\alpha=0.05)$

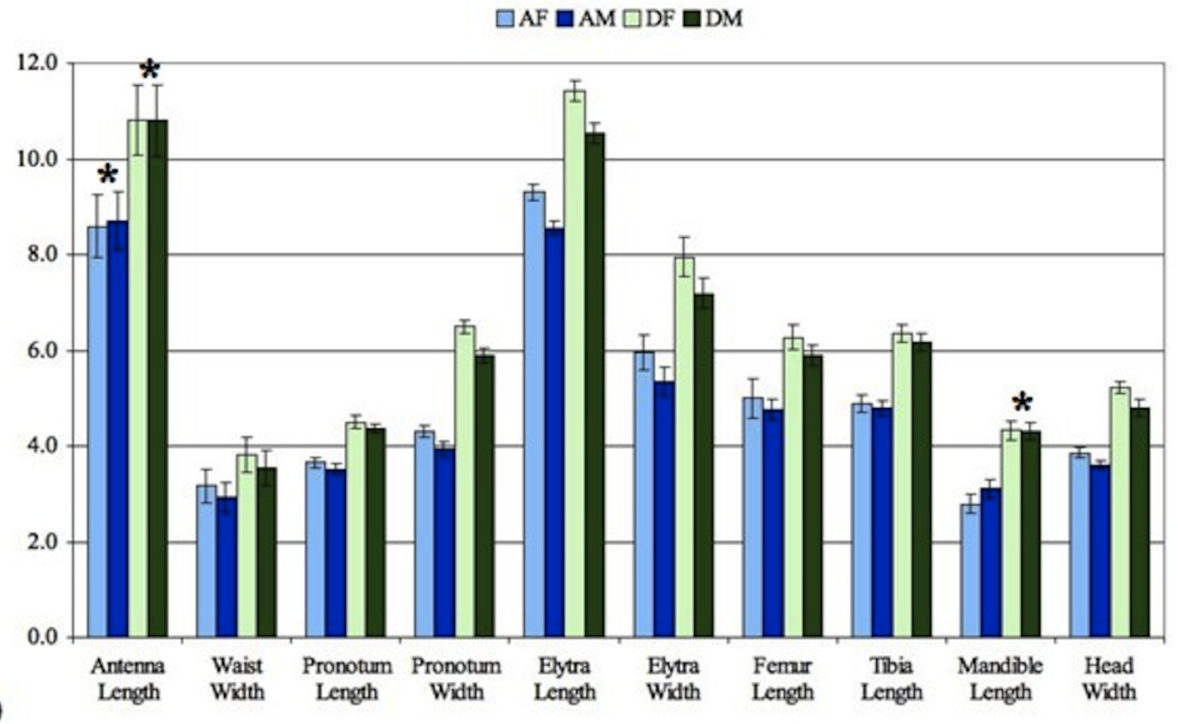

Figure 2.2: Univariate results of overall morphometric analysis of gross anatomy. Bars represent standard deviation. Characters indicated by an arrow and asterisk are not statistically different (REGWQ, $\mathrm{p}>0.05$ ). Also see Table 2.2 . 


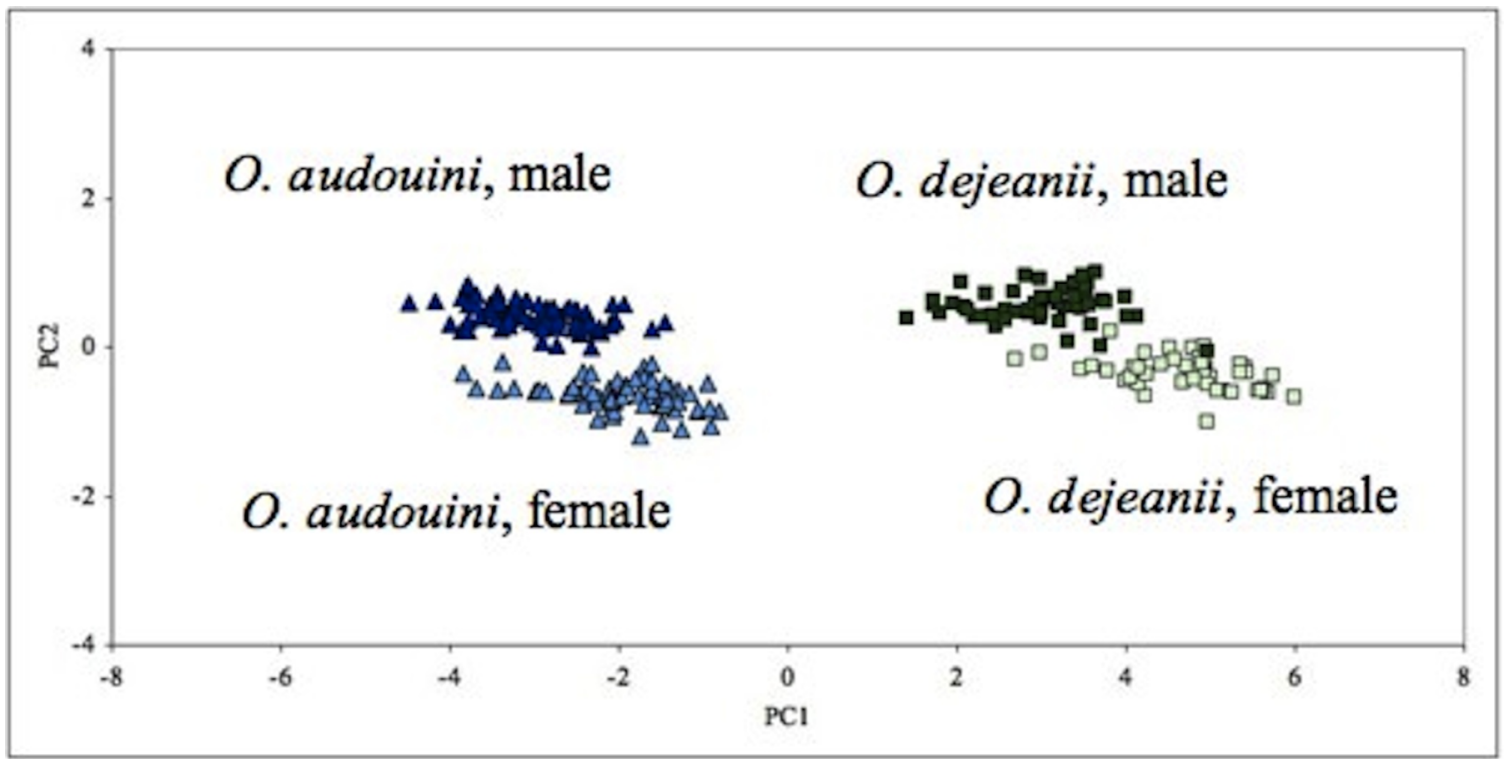

Figure 2.3: Results of the Principal Component Analysis (PCA) of gross anatomy for two species of Omus. See Table 2.5 for eigenvectors and eigenvalues of PCA. 


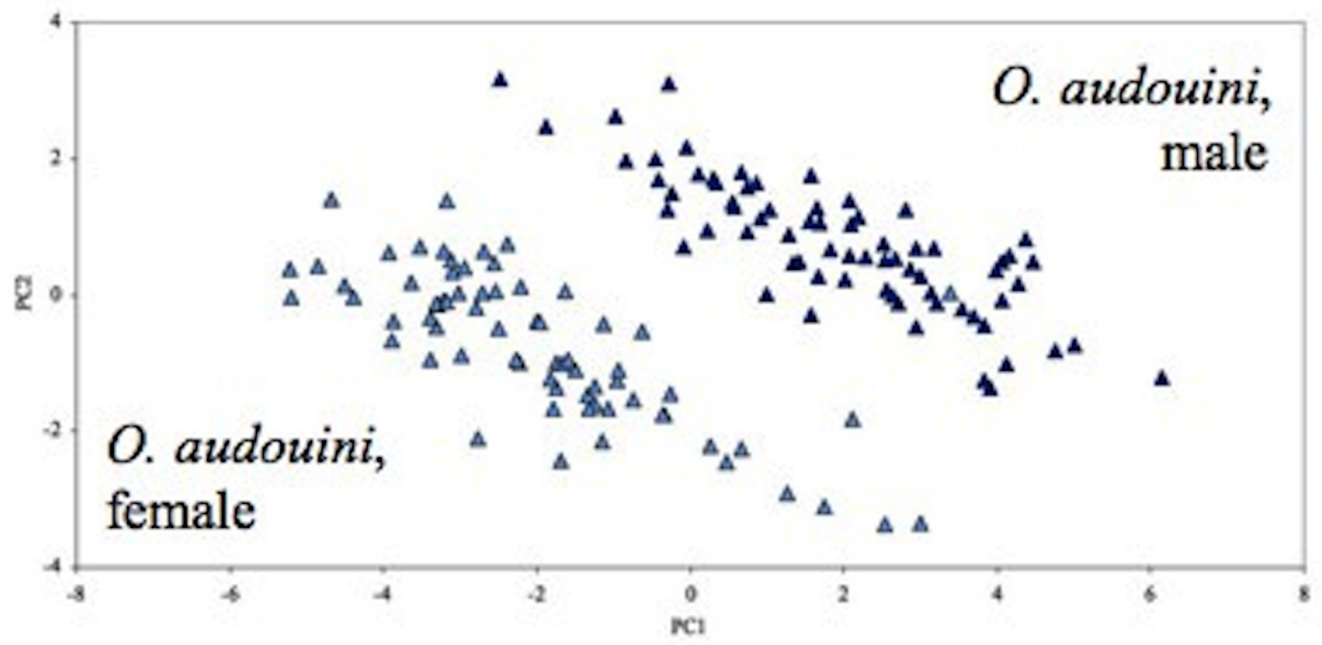

(A) PCA for Omus audouini

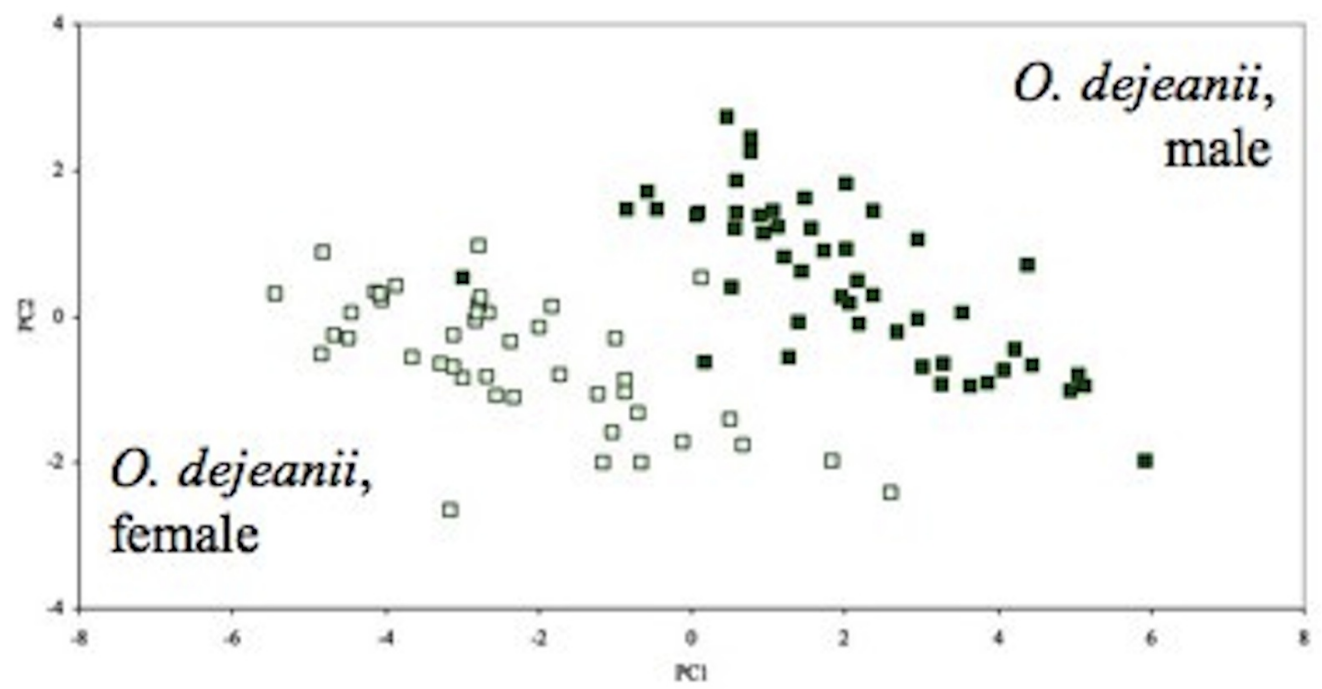

(B) PCA for Omus dejeanii.

Figure 2.4: Results of the Principal Component Analysis (PCA) of gross anatomy for two species of Omus. (A) O. audouini and (B) O. dejeanii. See Table 2.5 for eigenvectors and eigenvalues of PCA. 


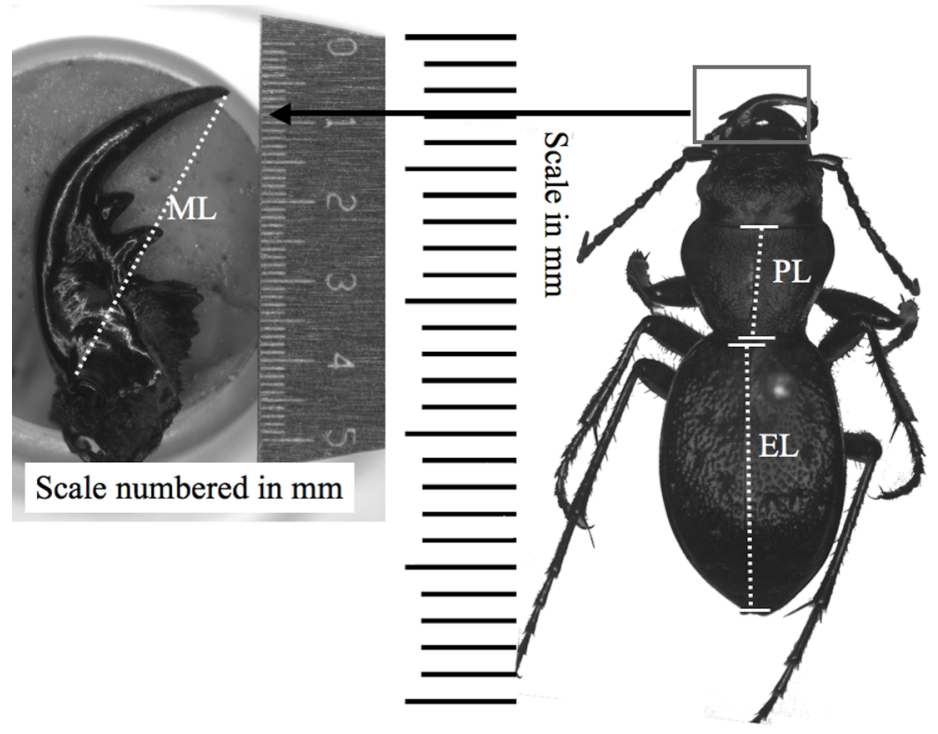

Figure 2.5: Linear measurements taken of left mandible (scale in millimeters) and pronotum (PL) and elytra (EL) (scale in millimeters). The sum of PL and EL was used as an alternative to overall body size for specimens (PEL). See text for details. 


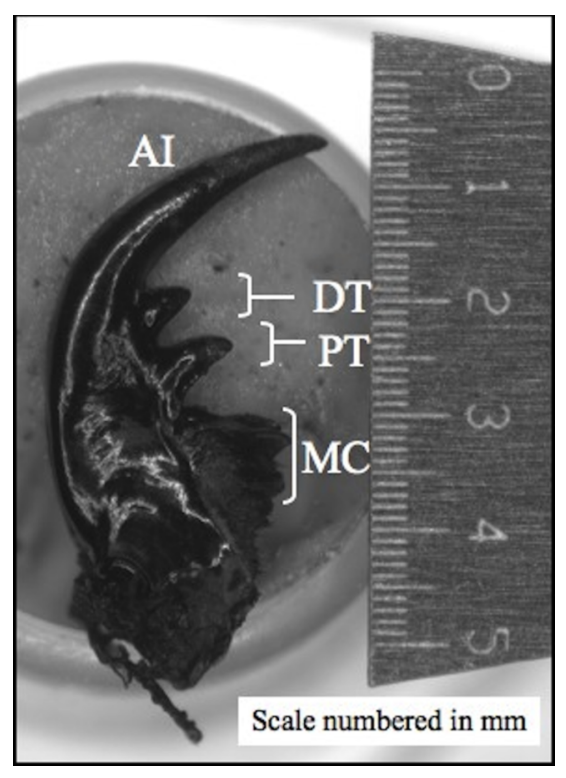

Figure 2.6: Left mandible of Omus dejeanii showing general mandible structures. AI = Apical incisor, $\mathrm{DT}=$ Distal terebral tooth, $\mathrm{PT}=$ Proximal terebral tooth, $\mathrm{MC}=$ Molar complex. 


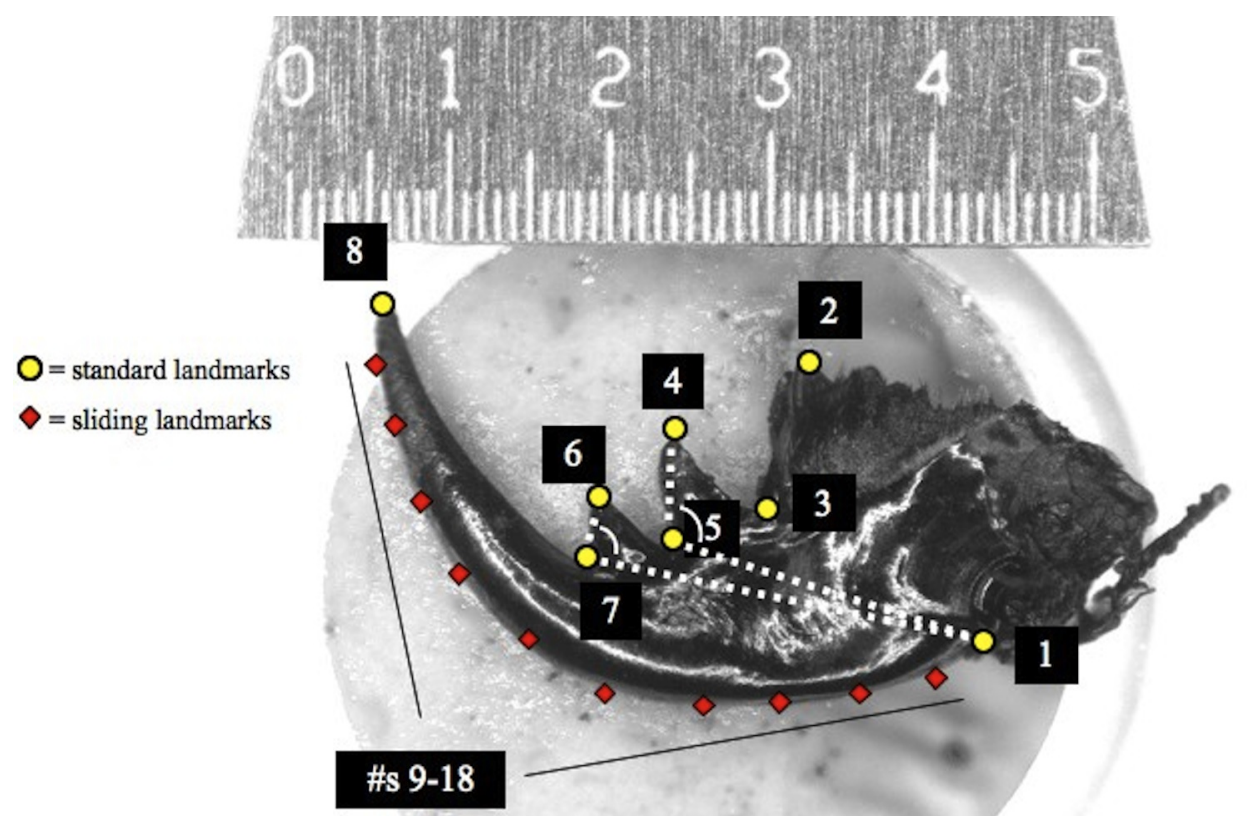

Figure 2.7: Geometric morphometric landmarks used in the mandibular analysis. Scale numbered in millimeters. See Table 3.2 for definitions. Dotted lines indicate measured angles for the proximal $(1,5,4)$ and distal $(1,7,6)$ terebral teeth. 


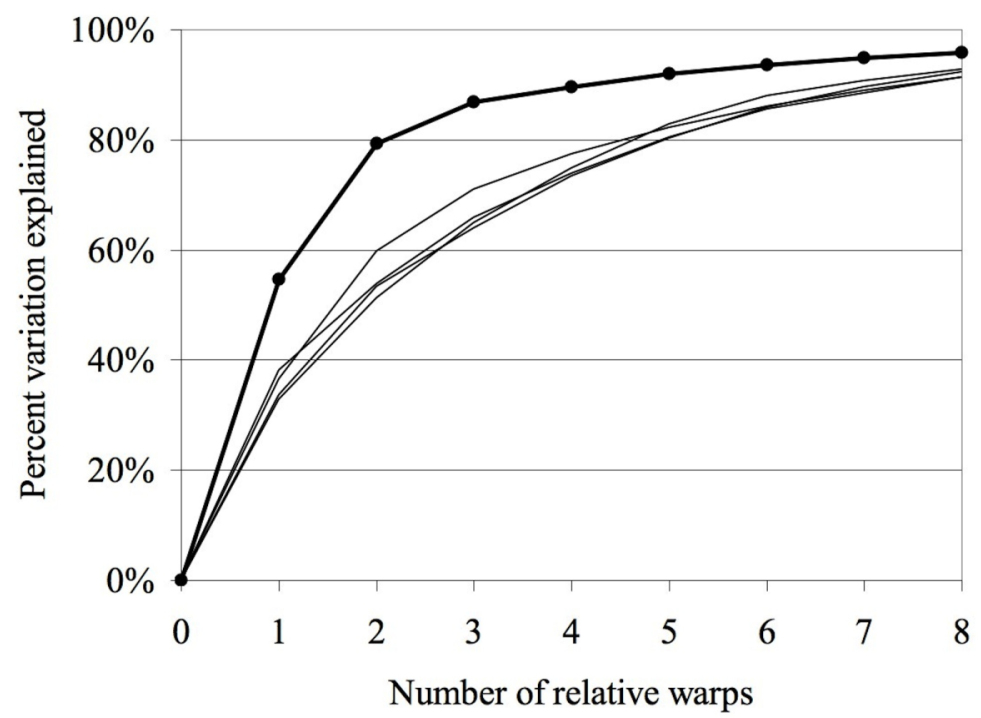

Figure 2.8: Percent variation among individuals explained by number of relative warps (by singular values, RWA analogue to PCA eigenvalues). The combined analysis results are represented by the thick line with points. The four single-category analyses had similar values, hence lines are not differentiated for clarity. 


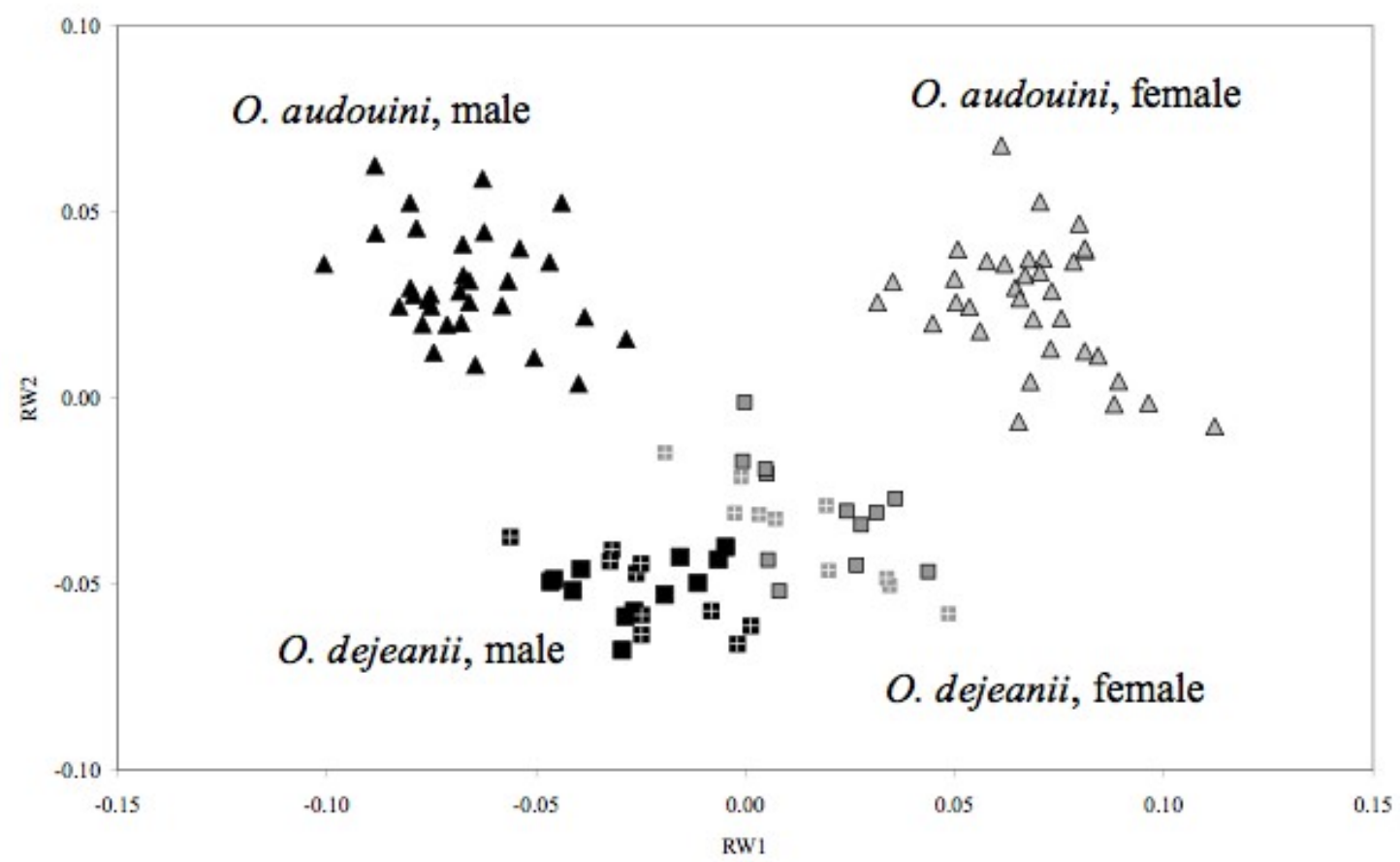

Figure 2.9: Relative warps analysis showing RW1 and RW2. RW1 separates the individuals primarily by sex. RW2 separates the individuals by species. Triangles represent $O$. audouini, Squares represent $O$. dejeanii. Black symbols indicate males, grey symbols indicate females. Square symbols with white crosses indicate $O$. dejeanii collected during the 2010 field season. All other specimens were collected during the 2012 field season. 


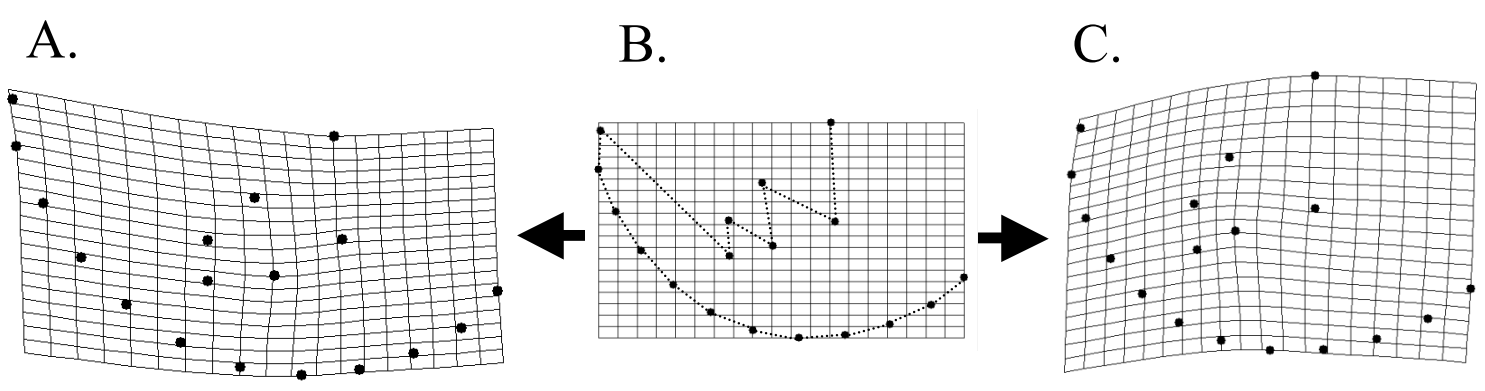

Figure 2.10: Thin-plate spline visualization of travel along RW1 ("sex"). (A) negative travel along RW1 results in a stereotypical non-specific "male" morphology with an elongated and straightened apical incisor, (B) consensus shape of a non-specific neuter (average shape, RW1 =0), (C) positive travel along RW1 results in a stereotypical nonspecific "female" morphology with a shorter and more curved apical incisor 


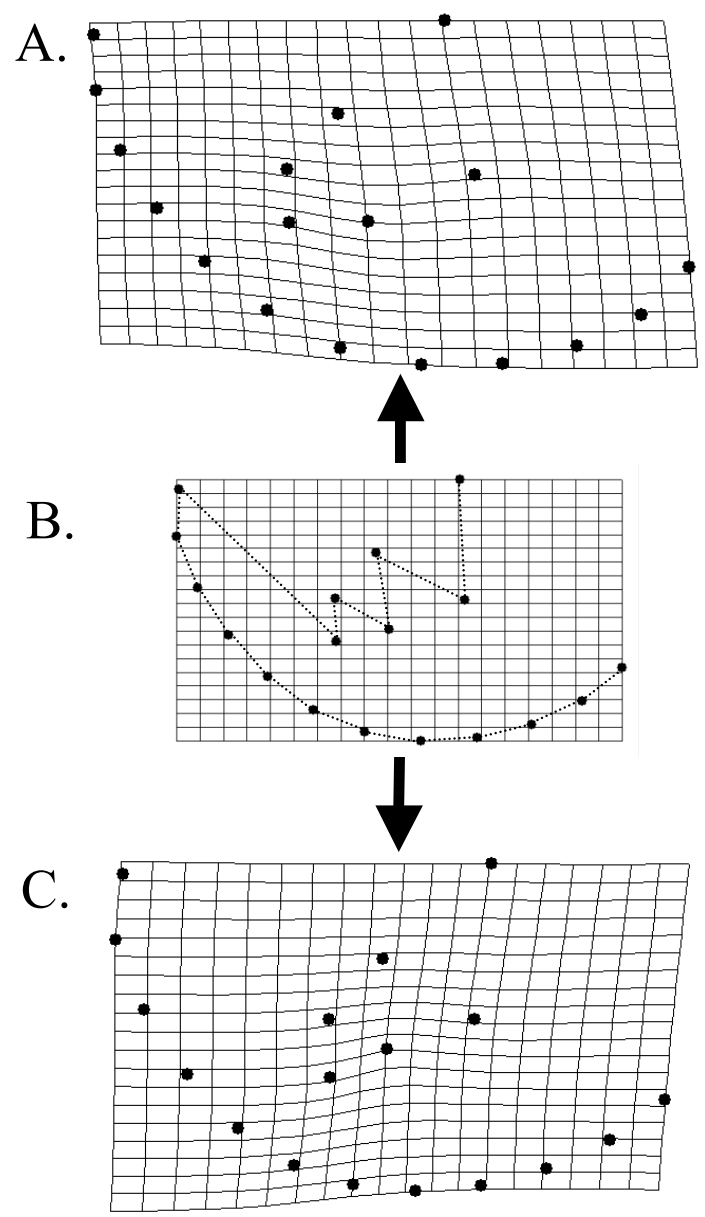

Figure 2.11: Thin-plate spline visualization of travel along RW2 ("species"). (A) positive travel along RW2 results in a stereotypical neuter $O$. audouini morphology with a more slender mandibular base and anteriorly-oriented terebral teeth, (B) consensus shape of a non-specific neuter (average shape, RW2 $=0$ ), $(\mathrm{C})$ negative travel along RW2 results in a stereotypical neuter $O$. dejeanii morphology with a more robust mandibular base and medially oriented terebral teeth. 


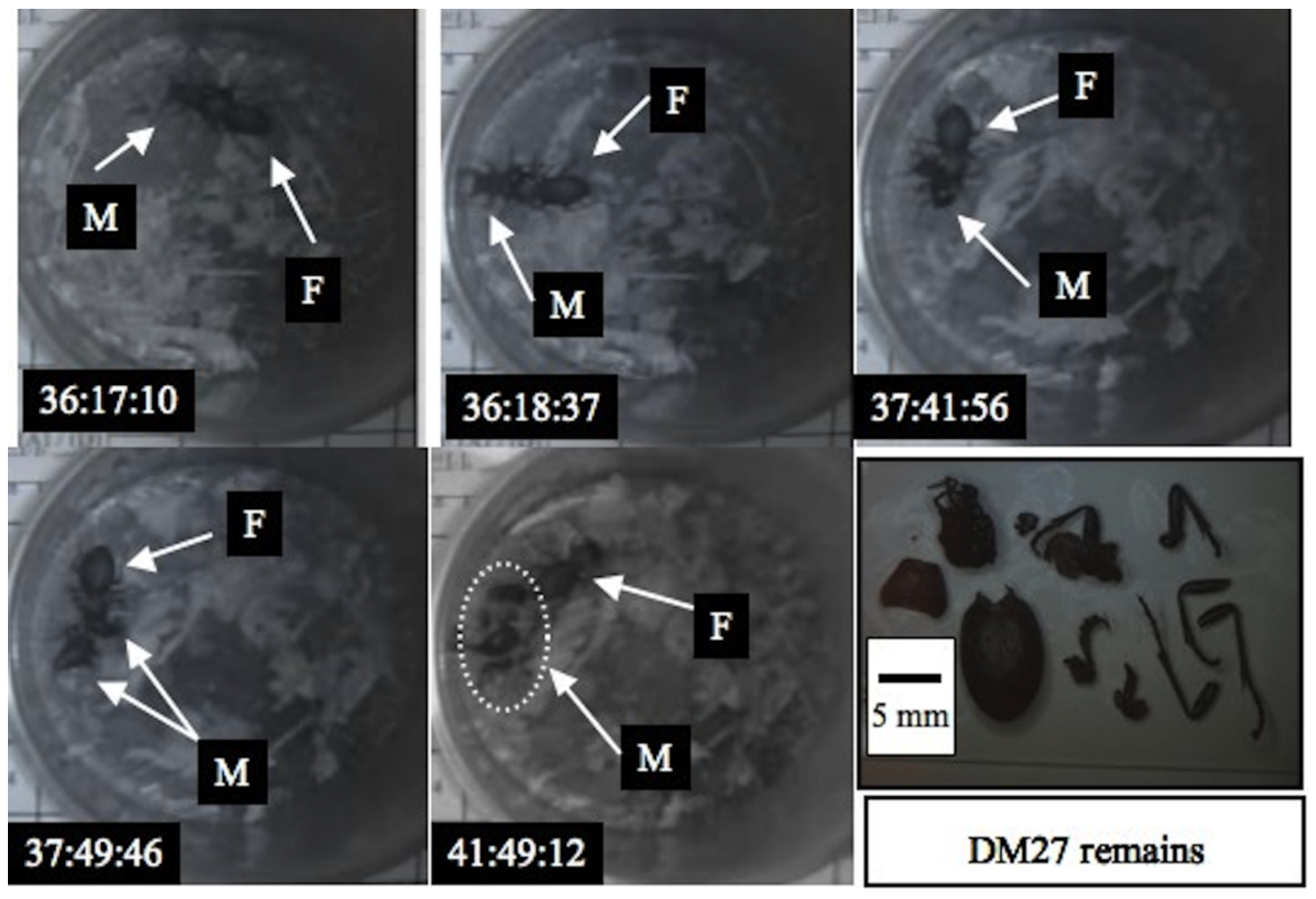

Figure 3.1: Screen-captures of post-copulatory intersexual cannibalism in Omus dejeanii and photograph of male remains (scale bar in photograph represents $5 \mathrm{~mm}$ ). 


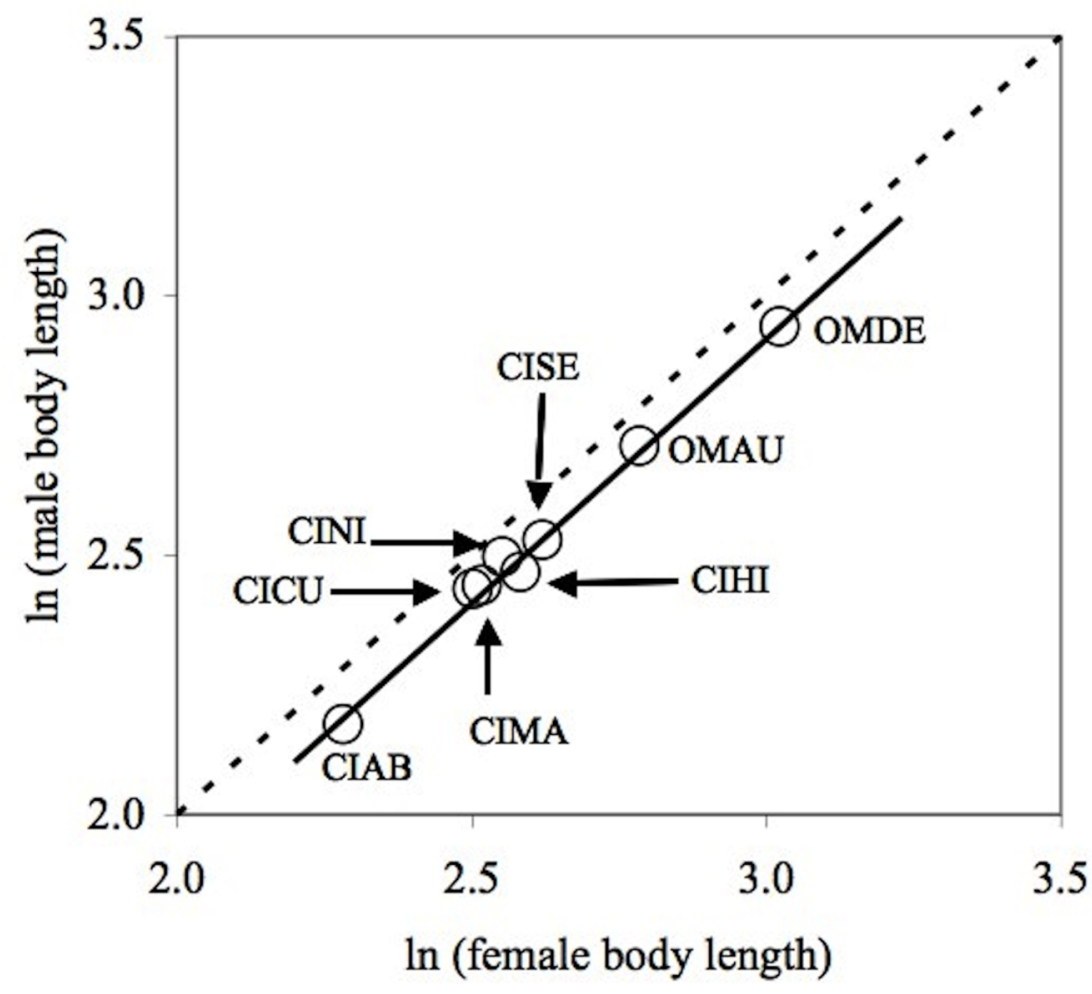

Figure 4.1: Trend of sexual size dimorphism across eight species of tiger beetles, reduced major axis regression of $\ln$ (male body length) onto $\ln$ (female body length). Species are abbreviated as per Table 4.2. Reduced major axis regression line: $\mathrm{y}=0.98 \mathrm{x}-0.14$, $\mathrm{r}^{2}=0.99$. Slope confidence interval (2.5-97.5\%): 0.89-1.07. Intercept confidence interval (2.5-97.5\%): $-0.08-0.35$. Dotted line indicates isometry $(\beta=1.0)$ for comparison. 


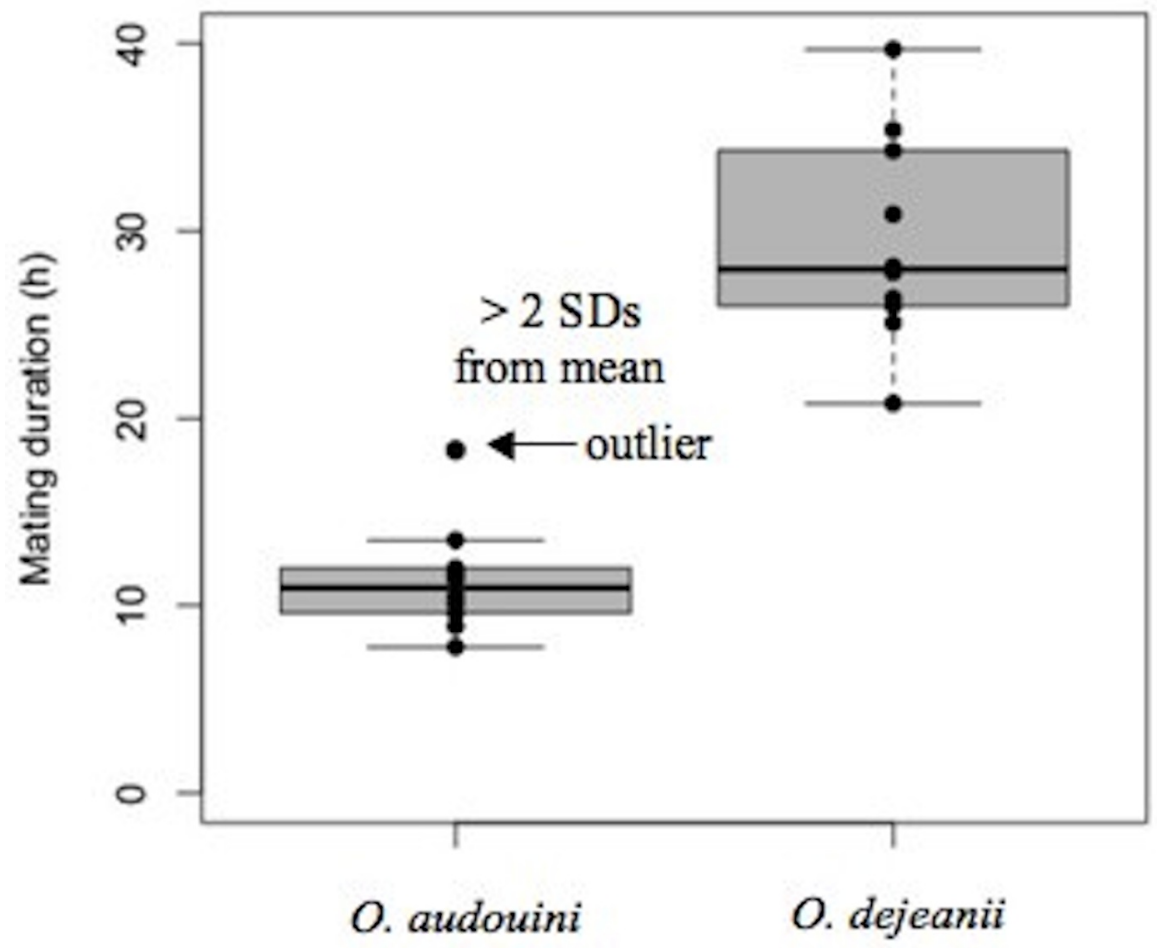

Figure 4.2: Box and whisker plot showing distribution of mating duration observations for $O$. audouini and $O$. dejeanii for time of initiation experiment (2011). Observation indicated by the arrow was a statistical outlier and not included in analyses. 


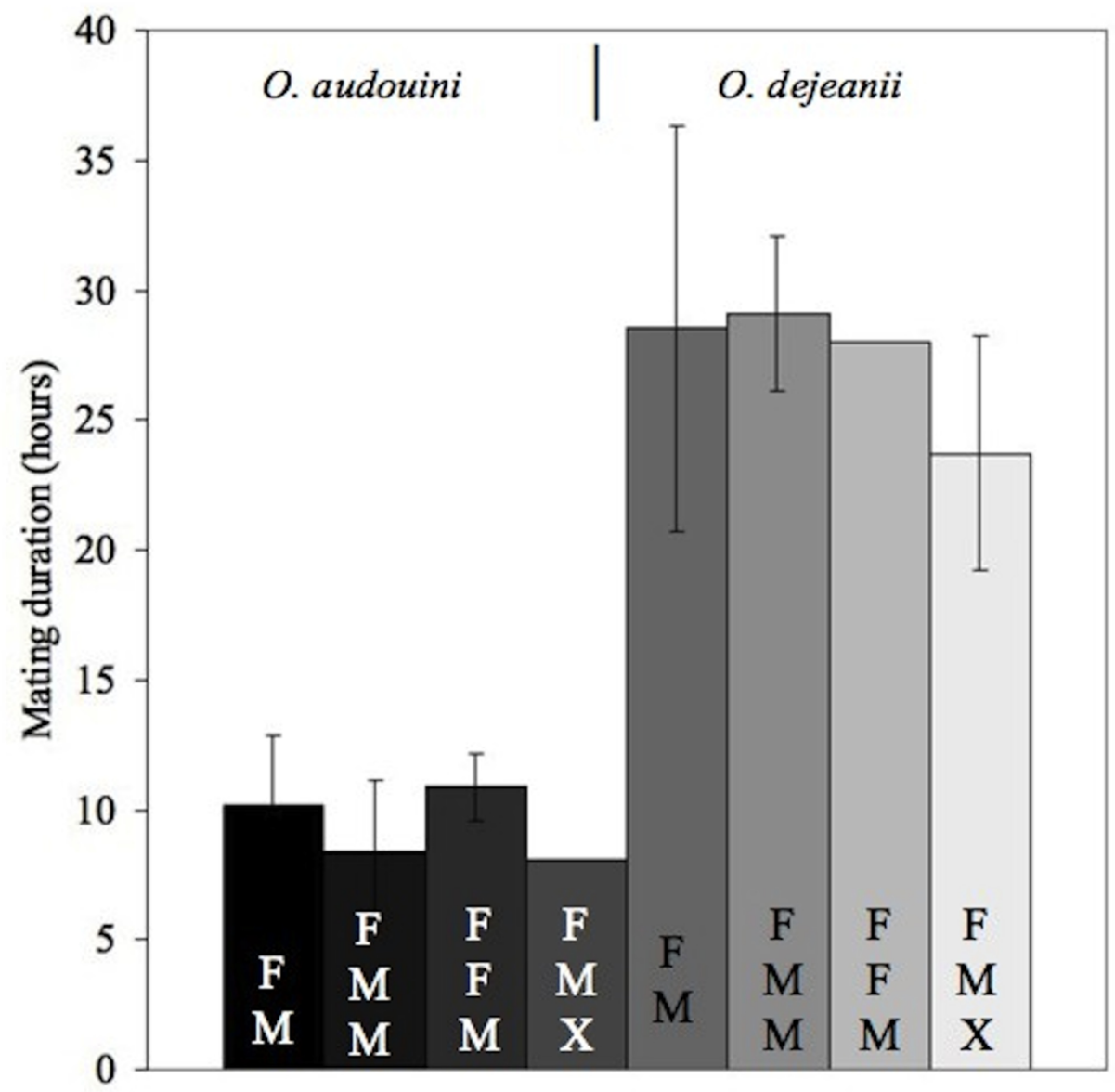

Figure 4.3: Mean mating duration (in minutes) for $O$. audouini and $O$. dejeanii under differing operational sex ratios. Bars represent $\mathrm{SD}$. FM = equal sex ratio, $\mathrm{FMM}=$ male biased sex ratio, FFM = female biased sex ratio, FMX = equal sex ratio but with a Scaphinotus sp. "witness". 


\section{Literature cited}

Abouheif, E., \& Fairbairn, D. J. (1997). A comparative analysis of allometry for sexual size dimorphism: assessing Rensch's rule. American Naturalist, 540-562.

Acorn, J. H., \& Ball, G. E. (1991). The mandibles of some adult ground beetles: structure, function, and the evolution of herbivory (Coleoptera: Carabidae). Canadian Journal of Zoology, 69(3), 638-650.

Adams, D. C., Rohlf, F. J., \& Slice, D. E. (2004). Geometric morphometrics: ten years of progress following the 'revolution'. Italian Journal of Zoology, 71(1), 5-16.

Alcock, J. (1994). Postinsemination associations between males and females in insects: the mate guarding hypothesis. Annual Review of Entomology, 39, 1-21.

Alcock, J. (2009) Animal behavior: An evolutionary approach (9th ed). Sinauer Associates, Inc, Sunderland, Massachusetts.

Aldridge, H. D. J. N., \& Rautenbach, I. L. (1987). Morphology, echolocation and resource partitioning in insectivorous bats. Journal of Animal Ecology, 763-778.

Altmann, J. (1974). Observational study of behavior: sampling methods. Behaviour, 227-267.

Andersson, M. (1994) Sexual selection. Princeton University Press, Princeton, New Jersey.

Angilletta Jr, M. J., \& Dunham, A. E. (2003). The temperature-size rule in ectotherms: simple evolutionary explanations may not be general. American Naturalist, 162(3), 332-342.

Arnqvist, G. R., \& Henriksson, S. T. E. F. A. N. (1997). Sexual cannibalism in the fishing spider and a model for the evolution of sexual cannibalism based on genetic constraints. Evolutionary Ecology, 11(3), 255-273.

Atkinson, D. (1996). Ectotherm life-history responses to developmental temperature. In Animals and temperature: phenotypic and evolutionary adaptation.:183-204. Johnston, I. A. \& Bennett, A. F. (Eds.). Cambridge University Press, New York, New York, USA.

Bagemihl, B. (1999). Biological exuberance: Animal homosexuality and natural diversity. New York: St. Martin's Press.

Baillie, J.E.M., Hilton-Taylor, C. and Stuart, S.N. (eds). (2004) 2004 IUCN Red List of Threatened Species. A Global Species Assessment. IUCN, Gland, Switzerland and Cambridge, UK.

Ball, G. E., Acorn, J. H., \& Shpeley, D. (2011). Mandibles and labrum-epipharynx of tiger beetles: basic structure and evolution (Coleoptera, Carabidae, Cicindelitae). ZooKeys, (147), 39-83.

Barraclough, T. G. \& Vogler, A. P. (2002). Recent diversification rates in North American tiger beetles estimated from a dated mtDNA phylogenetic tree. Molecular Biology and Evolution, 19, 1706-1716. 
Bartlett, J. (1987). Filial cannibalism in burying beetles. Behavioral Ecology and Sociobiology, 21(3), 179-183.

Bateman, A. J. (1948). Intra-sexual selection in Drosophila. Heredity, 2(3), 349-368.

Berglund, A., Rosenqvist, G., \& Svensson, I. (1986). Mate choice, fecundity and sexual dimorphism in two pipefish species (Syngnathidae). Behavioral Ecology and Sociobiology, 19(4), 301-307.

Birkhead, T. R. (1998). Cryptic female choice: criteria for establishing female sperm choice. Evolution, 1212-1218.

Birkhead, T. R. (2000). Promiscuity: an evolutionary history of sperm competition. Harvard University Press, Cambridge, Massachusetts.

Birkhead, T. R. (2010). How stupid not to have thought of that: post-copulatory sexual selection. Journal of Zoology, 281(2), 78-93.

Blanckenhorn, W. U. (2000). The evolution of body size: what keeps organisms small? Quartely Review Biology, 75, 385-407.

Blanckenhorn, W. U. (2005). Behavioral causes and consequences of sexual size dimorphism. Ethology 11, 977-1016.

Blanckenhorn, W. U., Dixon, A. F. G., Fairbairn, D. J., Foellmer, M. W., Gibert, P., van der Linde, K., Meier, R., Nylin, S., Pitnick, S., Schoff, C., Signorelli, M., Teder T. \& C. Wiklund, C. (2007a). Proximate causes of Rensch's rule: does sexual size dimorphism in arthropods result from sex differences in development time? American Naturalist, 169, 245-257.

Blanckenhorn, W. U., Meier, R. \& Teder, T. (2007b). Rensch's rule in insects: patterns among and within species. In Sex, size, and gender roles: evolutionary studies of sexual size dimorphism: 60-70. Fairbairn, D. J., Blanckenhorn, W. U. \& Székely, T., (Eds.). Oxford University Press, New York, New York.

Boggs, C. L. (1981). Nutritional and life-history determinants of resource allocation in holometabolous insects. American Naturalist, 692-709.

Bookstein, F. L. (1978). The measurement of biological shape and shape change. Lecture Notes in Biomathematics, Vol 24. Springer-Verlag, New York, New York.

Bookstein, F. L. (1986). Size and shape spaces for landmark data in two dimensions. Statistical Science, 181-222.

Bookstein, F. L. (1989). Principal warps: Thin-plate splines and the decomposition of deformations. Pattern Analysis and Machine Intelligence, IEEE Transactions on, 11(6), 567-585.

Bookstein, F. L., Chernoff, B., Elder, R. L., Humphries, J. M. Jr., Smith, G. R. \& Strauss, R. E. (1985). Morphometrics in evolutionary biology. Special publication 15, Academy of Natural Sciences of Philadelphia, Ann Arbor, Michigan. 
Brouat, C., Sennedot, F., Audiot, P., LeBlois, R. \& Rasplus, J. Y. (2003). Fine-scale genetic structure of two carabid species with contrasted levels of habitat specialization. Molecular Ecology 12, 1731-1745

Brown, W. L., \& Wilson, E. O. (1956). Character displacement. Systematic Zoology, 5(2), 49-64.

Buskirk, R. E., Frohlich, C., \& Ross, K. G. (1984). The natural selection of sexual cannibalism. American Naturalist, 612-625.

Cassola, F., \& Pearson, D. L. (2000). Global patterns of tiger beetle species richness (Coleoptera: Cicindelidae): their use in conservation planning. Biological Conservation, 95(2), 197-208.

Cazier, M. A. (1942). A monographic revision of the genus Omus (ColeopteraCicindelidae). PhD thesis (unpublished), University of California, Berkley.

Chapleau, K. M., \& Oswald, D. L. (2010). Power, sex, and rape myth acceptance: Testing two models of rape proclivity. Journal of Sex Research, 47(1), 66-78.

Chapman, A.D. (2009). Numbers of Living Species in Australia and the World, 2nd edition. Australian Biological Resources Study, Canberra.

Chiroro, P., Bohner, G., Viki, G. T., \& Jarvis, C. I. (2004). Rape Myth Acceptance and Rape Proclivity Expected Dominance Versus Expected Arousal as Mediators in Acquaintance-Rape Situations. Journal of Interpersonal Violence, 19(4), 427-442.

Christensen, B., \& Persson, L. (1993). Species-specific antipredatory behaviours: effects on prey choice in different habitats. Behavioral Ecology and Sociobiology, 32(1), 19.

Christy, J. H. (1995). Mimicry, mate choice, and the sensory trap hypothesis. American Naturalist, 171-181.

Cocroft, R. B., \& Rodriguez, R. L. (2005). The behavioral ecology of insect vibrational communication. Bioscience, 55(4), 323-334.

Connell, J. H. (1980). Diversity and the coevolution of competitors, or the ghost of competition past. Oikos, 131-138.

Connell, J. H. (1983). On the prevalence and relative importance of interspecific competition: evidence from field experiments. American Naturalist, 661-696.

Cornelisse, T. M. \& Hafernik, J. E. (2009). Effects of soil characteristics and human disturbance on tiger beetle oviposition. Ecological Entomology, 34, 495-503.

Cothran, R. D. (2004). Precopulatory mate guarding affects predation risk in two freshwater amphipod species. Animal Behaviour, 68(5), 1133-1138.

Cribb, B. W., Stewart, A., Huang, H., Truss, R., Noller, B., Rasch, R., \& Zalucki, M. P. (2008). Insect mandibles - comparative mechanical properties and links with metal incorporation. Naturwissenschaften, 95(1), 17-23. 
Daly, H. V. (1985). Insect morphometrics. Annual Review of Entomology, 30(1), 415438.

Darwin, C. (1859). On the origin of species by means of natural selection, or the preservation of favoured races in the struggle for life. John Murray, London, England. Facsimilie of the first edition published online: Wyhe, John van ed., 2002The Complete Work of Charles Darwin Online (http://darwin-online.org.uk/). accessed 25 Jan 2012.

Day, R. W., \& Quinn, G. P. (1989). Comparisons of treatments after an analysis of variance in ecology. Ecological Monographs, 59(4), 433-463.

Dayan, T., \& Simberloff, D. (2005). Ecological and community-wide character displacement: the next generation. Ecology Letters, 8(8), 875-894.

Drees, C., Matern, A., Rasplus, J., Terlutter, H., Assmann, T. \& Weber, F. (2008). Microsatellites and allozymes as the genetic memory of habitat fragmentation and defragmentation in populations of the ground beetle Carabus auronitens (Col., Carabidae). Journal of Biogeography, 35, 1937-1949

Dreisig, H. (1980). Daily activity, thermoregulation and water loss in the tiger beetle Cicindela hybrida. Oecologia, 44, 376-389.

Driscoll, D. A., Kirkpatrick, J. B., McQuillan, P. B., \& Bonham, K. J. (2010). Classic metapopulations are rare among common beetle species from a naturally fragmented landscape. Journal of Animal Ecology, 79(1), 294-303.

Eberhard, W. G. (1985). Sexual selection and animal genitalia. Harvard University Press, Cambridge, Massachusetts.

Eberhard, W. G. (1994). Evidence for widespread courtship during copulation in 131 species of insects and spiders, and implications for cryptic female choice. Evolution, $48,711-733$.

Eberhard, W. G. (1996). Female Control: Sexual selection by cryptic female choice. Princeton University Press, Princeton, N.J.

Elgar, M. A. \& Crespi, B. J. (1992). Ecology and evolution of cannibalism. In Elgar, M. A., \& Crespi, B. J. (eds) (1992). Cannibalism: ecology and evolution among diverse taxa. Oxford University Press, USA.

Elgar, M. A. (1992). Sexual cannibalism in spiders and other invertebrates. In Elgar, M. A., \& Crespi, B. J. (eds) (1992). Cannibalism: ecology and evolution among diverse taxa. Oxford University Press, USA.

Elton, C. S. (1927, this edition published in 2001) Animal Ecology. University of Chicago Press, Chicago, Illinois.

Elton, C. S., \& Miller, R. S. (1954). The ecological survey of animal communities: with a practical system of classifying habitats by structural characters. Journal of Ecology, 42(2), 460-496. 
Emlen, S. T., \& Oring, L. W. (1977). Ecology, sexual selection, and the evolution of mating systems. Science, 197(4300), 215-223.

Emlen, D. J., Marangelo, J., Ball, B., \& Cunningham, C. W. (2005). Diversity in the weapons of sexual selection: horn evolution in the beetle genus Onthophagus (Coleoptera: Scarabaeidae). Evolution, 59(5), 1060-1084.

Evans, M. E. G. (1965, June). The feeding method of Cicindela hybrida L. (Coleoptera: Cicindelidae). In Proceedings of the Royal Entomological Society of London. Series A, General Entomology (Vol. 40, No. 4-6, pp. 61-66). Blackwell Publishing Ltd.

Fairbairn, D. J. (1993). Costs of loading associated with mate-carrying in the waterstrider, Aquarius remigis. Behavioral Ecology, 4, 224-231.

Fairbairn, D. J. (1997). Allometry for sexual size dimorphism: pattern and process in the coevolution of body size in males and females. Annual Review of Ecology and Systematics, 659-687.

Fairbairn, D. J. (2007). Introduction: the enigma of sexual size dimorphism. In Sex, size, and gender roles: evolutionary studies of sexual size dimorphism: 60-70. Fairbairn, D. J., Blanckenhorn, W. U. \& Székely, T., (Eds.). Oxford University Press, New York, New York.

Fairbairn, D. J., Blanckenhorn, W. U. \& Székely, T., eds. (2007). Sex, size, and gender roles: evolutionary studies of sexual size dimorphism. Oxford University Press, New York, New York.

Farrell-Gray, C. C., \& Gotelli, N. J. (2005). Allometric exponents support a 3/4-power scaling law. Ecology, 86(8), 2083-2087.

Fenchel, T. (1975). Character displacement and coexistence in mud snails (Hydrobiidae). Oecologia, 20(1), 19-32.

Fielding, K. \& Knisley, C. B. (1995). Mating behavior in two tiger beetles, Cicindela dorsalis and C. puritana (Coleoptera; Cicindelidae). Entomological News 106(2), 61-67.

Figueirido, B., Palmqvist, P., \& Pérez-Claros, J. A. (2009). Ecomorphological correlates of craniodental variation in bears and paleobiological implications for extinct taxa: an approach based on geometric morphometrics. Journal of Zoology, 277(1), 70-80.

Fisher, R. A. (1915). The evolution of sexual preference. Eugenics Review, 7(3), 184192.

Fowler, D. W., Freedman, E. A., \& Scannella, J. B. (2009). Predatory functional morphology in raptors: interdigital variation in talon size is related to prey restraint and immobilisation technique. PloS one, 4(11), e7999.

Fox, L. R. (1975). Cannibalism in natural populations. Annual Review of Ecology and Systematics, 6, 87-106. 
Freitag, R. (1974). Selection for a non-genitalic mating structure in female tiger beetles of the genus Cicindela (Coleoptera: Cicindelidae). Canadian Entomologist 106, 561568.

Freitag, R. (1999). Catalogue of the tiger beetles of Canada and the United States. NRC Research Press, Ottawa, Ontario, Canada.

Freitag, R., Olynyk, J. E. \& Barnes, B. L. (1980). Mating behavior and genitalic counterparts in tiger beetles (Carabidae: Cicindelinae). International Journal of Invertebrate Reproduction, 2(2), 131-135.

Frost, S. R., Marcus, L. F., Bookstein, F. L., Reddy, D. P., \& Delson, E. (2003). Cranial allometry, phylogeography, and systematics of large-bodied papionins (primates: Cercopithecinae) inferred from geometric morphometric analysis of landmark data. Anatomical Record Part A: Discoveries in Molecular, Cellular, and Evolutionary Biology, 275(2), 1048-1072.

Futuyma, D. J., \& Moreno, G. (1988). The evolution of ecological specialization. Annual Review of Ecology and Systematics, 19, 207-233.

Galian, J., Hogan, J. E. \& Vogler, A. P. (2002). The origin of multiple sex chromosomes in tiger beetles. Molecular Biology and Evolution, 19(10), 1792-1796.

Gallup Jr, G. G., Burch, R. L., Zappieri, M. L., Parvez, R. A., Stockwell, M. L., \& Davis, J. A. (2003). The human penis as a semen displacement device. Evolution and Human Behavior, 24(4), 277-289.

Gamboa, G. J., Reeve, H. K., \& Pfennig, D. W. (1986). The evolution and ontogeny of nestmate recognition in social wasps. Annual Review of Entomology, 31(1), 431454.

Garnier, S., Alibert, P., Audiot, P., Prieur, B. \& Rasplus, J.Y. (2004). Isolation by distance and sharp discontinuities in gene frequencies: implications for the phylogeography of an alpine insect species, Carabus solieri. Molecular Ecology, 13, 1883-1897.

Gause, G. F. (1934). Experimental analysis of Vito Volterra's mathematical theory of the struggle for existence. Science, 79(2036), 16-17.

Gause, G. F. (1934, this edition 1964) the struggle for existence. Hafner Publishing Company, New York, New York.

Gillooly, J. F., Brown, J. H., West, G. B., Savage, V. M., \& Charnov, E. L. (2001). Effects of size and temperature on metabolic rate. Science, 293, 2248-2251.

Goldberg, D. E., \& Barton, A. M. (1992). Patterns and consequences of interspecific competition in natural communities: a review of field experiments with plants. American Naturalist, 771-801.

Gotelli, N. J. \& Ellison, A. M. (2004) A primer of ecological statistics. Sinauer Associates, Inc., Sunderland, Massachsetts, USA. 
Gould, S. J. \& Lewontin, R. C. (1979). The Spandrels of San Marco and the Panglossian Paradigm: A Critique of the Adaptationist Programme. Proceedings of the Royal Society of London. Series B, Biological Sciences, 205, 581-598.

Gould, S. J. (1966). Allometry and size in ontogeny and phylogeny. Biological Reviews, 41(4), 587-638.

Gould, S. J. (1974). The origin and function of' 'bizarre' structures: antler size and skull size in the 'Irish Elk,' Megaloceros giganteus. Evolution, 191-220.

Grant, P. R. (1986). Ecology and evolution of Darwin's finches. Princeton University Press, Princeton, New Jersey.

Grinnell, J. (1917). The niche-relationships of the California Thrasher. Auk, 34(4), 427433.

Gross, M. R. (1996). Alternative reproductive strategies and tactics: diversity within sexes. Trends in Ecology and Evolution, 11(2), 92-98.

Hardin, G. (1960). The competitive exclusion principle. Science, 131(3409), 1292-1297.

Hill, P. S. (2001). Vibration and animal communication: a review. American Zoologist, 41(5), 1135-1142.

Hoback, W. W., Golick, D. A., Svatos, T. M., Spomer, S. M. \& Higley, L. G. (2000). Salinity and shade preferences result in ovipositional differences between sympatric tiger beetle species. Ecological Entomology, 25, 180-187.

Hoback, W. W., Higley, L. G., \& Stanley, D. W. (2001). Tigers eating tigers: evidence of intraguild predation operating in an assemblage of tiger beetles. Ecological Entomology, 26(4), 367-375.

Horn, W. (1930). Notes on the races of Omus californicus and a list of the Cicindelidae of America north of Mexico (Coleoptera). Transactions of the American Entomological Society (1890-), 56(1), 73-86.

Hutchinson, G. E. (1957). Concluding remarks. Cold Springs Harbor Symposium of Quantitative Biology, 22, 415-427.

Hutchinson, G. E. (1959). Homage to Santa Rosalia or why are there so many kinds of animals? American Naturalist, 93, 145-159.

Johnson, K. (1988). Sexual selection in pinyon jays II: male choice and female-female competition. Animal Behaviour, 36, 1048-1053.

Kaulenas, M. S. (1992). Insect accessory reproductive structures. Springer-Verlag, New York, New York, USA.

Kavanaugh, D. H., \& LaBonte, J. R. (2008). Discovery of Nebria Brevicollis (Fabricius) (Coleoptera: Caribidae: Nebriini), a European Ground Beetle, Established in the Willamette Valley, Oregon. California Academy of Sciences. Proceedings of the California Academy of Sciences, Series 4, 59, 481-488. 
Keller, I., Nentwig, W. \& Largiader, C.R. (2004). Recent habitat fragmentation due to roads can lead to significant genetic differentiation in an abundant flightless ground beetle. Molecular Ecology, 13, 2983-2994.

Kim, K. W., Roland, C., \& Horel, A. (2000). Functional value of matriphagy in the spider Amaurobius ferox. Ethology, 106(8), 729-742.

Kingston, T., \& Rossiter, S. J. (2004). Harmonic-hopping in Wallacea's bats. Nature, 429(6992), 654-657.

Klowden, M. K. (2007). Physiological systems in insects. Academic Press/Elsevier, Burlington, MA.

Knisley, C. B. \& Haines, D. R. (2010). Distribution and conservation status of Omus submetallicus G. Horn and its confusion with Omus californicus lecontei G. Horn (Coleoptera: Carabidae). Coleopterists Bulletin, 64(3), 243-248.

Knisley, C. B. \& Juliano, S. A. (1988). Survival, development, and size of larval tiger beetles: effects of food and water. Ecology, 69(6), 1983-1992.

Knisley, C. B. \& Schultz, T. D. (1997). The biology of tiger beetles and a guide to the species of the South Atlantic states. Virginia Museum of Natural History, Martinsville, Virginia.

Knisley, C. B. (1987). Habitats, food resources, and natural enemies of a community of larval Cicindela in southeastern Arizona (Coleoptera: Cicindelidae). Canadian Journal of Zoology, 65(5), 1191-1200.

Koehl, M. A. R. (1996). When does morphology matter?. Annual Review of Ecology and Systematics, 501-542.

Kraus, B., \& Lederhouse, R. C. (1983). Contact guarding during courtship in the tiger beetle Cicindela marutha Dow (Coleoptera: Cicindelidae). American Midland Naturalist, 208-211.

Kritsky, G., \& Simon, S. (1995). Mandibular sexual dimorphism in Cicindela Linnaeus (Coleoptera: Cicindelidae). Coleopterists Bulletin, 143-148.

Kronfeld-Schor, N., \& Dayan, T. (2003). Partitioning of time as an ecological resource. Annual Review of Ecology, Evolution, and Systematics, 153-181.

LaBonte, J.R. \& Johnson, P. J. (1988). Millipede predation by Omus dejeanii Reiche. Cicindela, 20, 53-54.

Lack, D. (1947, this edition published 1961) Darwin's finches. Harper \& Brothers, New York, NY.

Lank, D. B., Smith, C. M., Hanotte, O., Burke, T., \& Cook, F. (1995). Genetic polymorphism for alternative mating behaviour in lekking male ruff. Nature, 378, 59-62.

Lawton, J. H. \& Strong, D. R. (1981). Community Patterns and Competition in Folivorous Insects. American Naturalist, 118, 317-338. 
Leffler, S. R. (1979). Tiger beetles of the Pacific Northwest. PhD thesis (unpublished). University of Washington, Seattle, WA.

Leffler, S. R., Nelson, R. E. \& van den Berghe, E. (1986). Color variation and sex ratio in Omus dejeani Reiche. Cicindela, 18, 7-11.

Lloyd, D. G., \& Webb, C. J. (1977). Secondary sex characters in plants. Botanical Review, 43(2), 177-216.

Lomolino, M. V. (1985). Body size of mammals on islands: the island rule reexamined. American Naturalist, 125(2), 310-316.

Lomolino, M. V., Riddle, B. R. \& Brown, J. H. (2006). Biogeography, 3rd ed. Sinauer Associates, Inc., Sunderland, Massachusetts.

Losos, J. B. \& Miles, D.B. 1994. Adaptation, constraint and the comparative method: Phylogenetic issues and methods. Pages 60-98 in Wainwright, P.C. and S.M. Reilly. 1994. Ecological morphology: integrative organismal biology. University of Chicago Press, Chicago, IL.

Lovei, G. L. \& Sunderland, K. D. (1996). Ecology and behavior of ground beetles (Coleoptera: Carabidae). Annual Review of Entomology, 41, 231-256.

Lovich, J. E., \& Gibbons, J. W. (1992). A review of techniques for quantifying sexual size dimorphism. Growth, development, and aging: GDA, 56(4), 269-281.

Luckinbill, L. S. (1974). The effects of space and enrichment on a predator-prey system. Ecology, 1142-1147.

MacArthur, R. H., \& Pianka, E. R. (1966). On optimal use of a patchy environment. American Naturalist, 603-609.

Maddison, D. R., Moore, W., Baker, M. D., Ellis, T. M., Ober, K. A., Cannone, J. J., \& Gutell, R. R. (2009). Monophyly of terrestrial adephagan beetles as indicated by three nuclear genes (Coleoptera: Carabidae and Trachypachidae). Zoologica Scripta, 38(1), 43-62.

Maddison, D., Baker, M. D., \& Ober, K. (1999). Phylogeny of carabid beetles as inferred from 18S ribosomal DNA (Coleoptera: Carabidae). Systematic Entomology, 24(2), 103-138.

Manning, A. (1967). The control of sexual receptivity in female Drosophila. Animal Behaviour, 15(2), 239-250.

Maser, C. \& Beer, F. M. (1971). Notes on the daily activity of Omus audouini and Omus dejeani. Cicindela 3, 52.

Maser, C. (1977a). Notes on Omus dejeani. Cicindela, 9(2), 35-38.

Maser, C. (1977b). Notes on Omus audouini. Cicindela, 9(3), 47-49.

Maser, C., \& Hooven, E. F. (1974). Notes on the behavior and food habits of captive Pacific Shrews, Sorex pacificus pacificus. Northwest Science, 48, 81-95. 
May, M. L., Pearson, D. L. \& Casey, T. M. (1986). Oxygen consumption of active and inactive adult tiger beetles. Physiological Entomology, 11, 171-179.

McIver, S. B. (1975). Structure of cuticular mechanoreceptors of arthropods. Annual Review of Entomology, 20(1), 381-397.

Mousseau, T. A. (1997). Ectotherms follow the converse to Bergmann's rule. Evolution, $51,630-632$.

Mury Meyer, E. J. (1981). The Capture Efficiency of Flickers Preying on Larval Tiger Beetles. Auk, 98, 189-191.

Owens, I. P., Burke, T., \& Thompson, D. B. (1994). Extraordinary sex roles in the Eurasian dotterel: female mating arenas, female-female competition, and female mate choice. American Naturalist, 76-100.

Parker, G. A. (1970). Sperm competition and its evolutionary consequences in the insects. Biological Reviews, 45(4), 525-567.

Parker, G. A. (1974). Courtship persistence and female-guarding as male time investment strategies. Behaviour 48, 157-184.

Parker, G. A., Baker, R. R. \& Smith, V. G. (1972). The origin and evolution of gamete dimorphism and the male-female phenomenon. Journal of Theoretical Biology, 36, 529-553.

Pearson, D. L. \& Cassola, F. (2005). A quantitative analysis of species descriptions of tiger beetles (Coleoptera: Cicindelidae), from 1758 to 2004, and notes about related developments in biodiversity studies. Coleopterists Bulletin, 59(2), 184-193.

Pearson, D. L. \& Juliano, S. A. (1991). Mandible length ratios as a mechanism for cooccurrence: evidence from a world-wide comparison of tiger beetle assemblages (Cicindelidae). Oikos, 61, 223-233.

Pearson, D. L. \& Mury, E. J. (1979). Character divergence and convergence among tiger beetles (Coleoptera: Cicindelidae). Ecology, 60, 557-566.

Pearson, D. L. \& Vogler, A. P. (2001). Tiger beetles: the evolution, ecology, and diversity of the Cicindelids. Cornell University Press, Ithaca, New York, USA.

Pearson, D. L. (1980). Patterns of Limiting Similarity in Tropical Forest Tiger Beetles (Coleoptera: Cicindelidae). Biotropica, 12, 195-204.

Pearson, D. L. (1990). The evolution of multi anti-predator characteristics as illustrated by tiger beetles (Coleoptera: Cicindelidae). Florida Entomologist, 67-70.

Pearson, D. L., \& Cassola, F. (2007). Are we doomed to repeat history? A model of the past using tiger beetles (Coleoptera: Cicindelidae) and conservation biology to anticipate the future. Journal of Insect Conservation, 11(1), 47-59.

Pearson, D. L., Knisley, C. B. \& Kazilek, C. J. (2006). A field guide to the tiger beetles of the United States and Canada. Oxford University Press, New York, New York. 
Pechenik, J. A. (2010). Biology of the invertebrates (6th ed), McGraw-Hill, Dubuque, Iowa.

Persson, L., \& Eklöv, P. (1995). Prey refuges affecting interactions between piscivorous perch and juvenile perch and roach. Ecology, 70-81.

Polis, G. A., Myers, C. A. \& Holt, R. D. (1989). The Ecology and Evolution of Intraguild Predation: Potential Competitors That Eat Each Other. Annual Review of Ecology and Systematics, 20, 297-330.

Pratt, R. Y. (1939). The mandibles of Omus dejeanii Riche as secondary sexual organs (Coleoptera, Cicindelidae.). Pan-Pacific Entomologist 15, 95-96.

Pyke, G. H. (1984). Optimal foraging theory: a critical review. Annual Review of Ecology and Systematics, 15, 523-575.

R Core Development Team (2011). R: A language and environment for statistical computing. R Foundation for Statistical Computing, Vienna, Austria. ISBN 3900051-07-0,http://www.R-project.org/.

Reeve, J. P. \& Fairbairn, D. J. (1996). Sexual Size Dimorphism as a Correlated Response to Selection on Body Size: An Empirical Test of the Quantitative Genetic Model. Evolution, 50, 1927-1938.

Rensch, B. (1960). Evolution above the species level. Columbia University Press, Morningside Heights, New York.

Renwick, J. A. A. (1989). Chemical ecology of oviposition in phytophagous insects. Experientia, 45, 223-228.

Richardson, R. K. (2010). Mandibular chirality in tiger beetles (Carabidae: Cicindelinae). Coleopterists Bulletin, 64(4), 386-387.

Rodriguez, R. L. (1998). Mating behavior of two Pseudoxychila beetles (Coleoptera: Cicindelidae). Canadian Entomologist, 130(06), 735-750.

Rodriguez, R. L. (1999). Spermatophore transfer and ejection in the beetle Pseudoxychila tarsalis (Coleoptera: Cicindelidae). Journal of the Kansas Entomological Society, 72(1), 1-9.

Rohlf, F. J. \& Archie, J. W. (1984). A comparison of Fourier methods for the description of wing shape in mosquitoes (Diptera: Culicidae). Systematic Biology, 33, 302-317.

Rohlf, F. J. \& Bookstein, F. L. (2003). Computing the uniform component of shape variation. Systematic Biology, 52, 66-69.

Rohlf, F. J. \& Marcus, L. F. (1993). A revolution morphometrics. Trends in Ecology and Evolution, 8, 129-132.

Rohlf, F. J. (1990). Morphometrics. Annual Review of Ecology and Systematics. 21, 299-316.

Rohlf, F. J. (1999). Shape statistics: Procrustes superimpositions and tangent spaces. Journal of Classification, 16, 197-223. 
Rohlf, F. J. (2010a). tpsDig, version 2.16. Department of Ecology and Evolution, State University of New York at Stony Brook.

Rohlf, F. J. (2010b). tpsRelw, version 1.49. Department of Ecology and Evolution, State University of New York at Stony Brook.

Rohlf, F. J. (2011). tpsRegr, v1.38. Department of Ecology and Evolution, State University of New York at Stony Brook.

Rohlf, F. J. (2012). tpsUtil, v1.49. Department of Ecology and Evolution, State University of New York at Stony Brook.

Rosenqvist, G. (1990). Male mate choice and female-female competition for mates in the pipefish Nerophis ophidion. Animal Behaviour, 39, 1110-1115.

Rowe, L. (1994). The costs of mating and mate choice in water striders. Animal Behaviour, 48(5), 1049-1056.

RStudio (2012). RStudio: Integrated development environment for R (Version 0.96.122) [Computer software]. Boston, MA. Retrieved 23 May 2012; Available from http://www.rstudio.org/

Ruedas, L. A., Demboski, J. R., \& Sison, R. V. (1994). Morphological and ecological variation in Otopteropus cartilagonodus Knock, 1969 (Mammalia: Chiroptera: Pteropodidae) from Luzon, Philippines. Proceedings of the Biological Society of Washington, 107(1), 1-16.

Saeki, Y., Kruse, K. C. \& Switzer, P. V. (2005). Physiological costs of mate guarding in the Japanese beetle (Popillia japonica Newman). Ethology, 111, 863-877.

Scheffer, T. H. (1911). Investigation of the mole. Transactions of the Kansas Academy of Science (1903), 119-131.

Schoener, T. W. (1974). Resource partitioning in ecological communities. Science, 185, 27-39.

Schoener, T. W. (1983). Field experiments on interspecific competition. American Naturalist, 122, 240-285.

Schultz, T. D., Quinlan, M. C., \& Hadley, N. F. (1992). Preferred body temperature, metabolic physiology, and water balance of adult Cicindela longilabris: a comparison of populations from boreal habitats and climatic refugia. Physiological Zoology, 226-242.

Shapiro, A. M., \& Porter, A. H. (1989). The lock-and-key hypothesis: evolutionary and biosystematic interpretation of insect genitalia. Annual Review of Entomology, 34(1), 231-245.

Shine, R. (1989). Ecological causes for the evolution of sexual dimorphism: a review of the evidence. Quarterly Review of Biology, 419-461.

Shivashankar, T. \& Pearson, D. L. (1994). A comparison of mate guarding among five syntopic tiger beetle species from peninsular India (Coleoptera: Cicindelidae).

Biotropica 26, 436-442. 
Shull, V. L., Vogler, A. P., Baker, M. D., Maddison, D. R., \& Hammond, P. M. (2001). Sequence alignment of $18 \mathrm{~S}$ ribosomal RNA and the basal relationships of adephagan beetles: evidence for monophyly of aquatic families and the placement of Trachypachidae. Systematic Biology, 50(6), 945-969.

Shuster, S. M. (1987). Alternative reproductive behaviors: three discrete male morphs in Paracerceis sculpta, an intertidal isopod from the northern Gulf of California. Journal of Crustacean Biology, 318-327.

Shuster, S. M., \& Wade, M. J. (1991). Equal mating success among male reproductive strategies in a marine isopod. Nature, 350(6319), 608-610.

Siemers, B. M., \& Schnitzler, H. U. (2004). Echolocation signals reflect niche differentiation in five sympatric congeneric bat species. Nature, 429(6992), 657-661.

Sih, A., Crowley, P., McPeek, M., Petranka, J., \& Strohmeier, K. (1985). Predation, competition, and prey communities: a review of field experiments. Annual Review of Ecology and Systematics, 16, 269-311.

Simberloff, D. \& Boecklen, W. (1981). Santa Rosalia Reconsidered: Size Ratios and Competition. Evolution, 35, 1206-1228.

Simmons, L. W. (2001). Sperm competition and its evolutionary consequences in insects. Princeton University Press, Princeton, New Jersey, USA.

Singer, T. L. (1998). Roles of hydrocarbons in the recognition systems of insects. American Zoologist, 38(2), 394-405.

Slice, D. E. (2001). Landmark coordinates aligned by Procrustes analysis do not lie in Kendall's shape space. Systematic Biology, 50, 141-149.

Slice, D. E. (2007). Geometric morphometrics. Annual Review of Anthropology, 36, 261-281.

Sota, T. \& Kubota, K. (1998). Genital Lock-and-Key as a Selective Agent against Hybridization. Evolution, 52, 1507-1513.

Stevens, L. (1992). Cannibalism in beetles. In Elgar, M. A., \& Crespi, B. J. (eds) (1992). Cannibalism: ecology and evolution among diverse taxa. Oxford University Press, USA.

Suckling, D. M., Gibb, A. R., Daly, J. M., Chen, X., \& Brockerhoff, E. G. (2001). Behavioral and electrophysiological responses of Arhopalus tristis to burnt pine and other stimuli. Journal of Chemical Ecology, 27(6), 1091-1104.

Sueur, J. (2002). Cicada acoustic communication: potential sound partitioning in a multispecies community from Mexico (Hemiptera: Cicadomorpha: Cicadidae). Biological Journal of the Linnean Society, 75(3), 379-394.

Suzuki, S., Kitamura, M., \& Matsubayashi, K. (2005). Matriphagy in the hump earwig, Anechura harmandi (Dermaptera: Forficulidae), increases the survival rates of the offspring. Journal of Ethology, 23(2), 211-213. 
Takami, Y. (2007). Spermatophore displacement and male fertilization success in the ground beetle Carabus insulicola. Behavioral Ecology, 18(3), 628-634.

Talarico, F., Romeo, M., Massolo, A., Brandmayr, P., \& Zetto, T. (2007). Morphometry and eye morphology in three species of Carabus (Coleoptera: Carabidae) in relation to habitat demands. Journal of Zoological Systematics and Evolutionary Research, 45(1), 33-38.

Teder, T. \& Tammaru, T. (2005). Sexual size dimorphism within species increases with body size in insects. Oikos, 108, 321-334.

Thiele, H. U. (1977). Carabid beetles in their environments: a study on habitat selection by adaptations in physiology and behaviour. Springer-Verlag, Berlin.

Thompson, D. W. (1917, this edition published in 1969). On growth and form. Cambridge University Press, New York, New York.

Thornhill, R. \& Alcock, J. (1983). The evolution of insect mating systems. Harvard University Press, Cambridge, Massachusetts.

Thornhill, R. (1976). Sexual Selection and Paternal Investment in Insects. American Naturalist, 110, 153-163.

Thornhill, R. (1983). Cryptic female choice and its implications in the scorpionfly Harpobittacus nigriceps. American Naturalist, 765-788.

Tizo-Pedroso, E. and Del-Claro, K. (2005). Matriphagy in the Neotropical Pseudoscorpion Paratemnoides nidificator (Balzan 1888) (Atemnidae). Journal of Arachnology, 33, 873-877.

Toft, C. (1985). Resource Partitioning in Amphibians and Reptiles. Copeia,1985, 1-21.

Toyama, M. (1999). Adaptive advantages of maternal care and matriphagy in a foliage spider, Chiracanthium japonicum (Araneae: Coubionidae). Journal of Ethology, 17(1), 33-39.

Triplehorn, C. A. \& Johnson, N. F. (2007). Borror and DeLong's introduction to the study of insects, 7th ed. Thomson Brooks/Cole, Belmont California, USA.

Trivers, R. L. (1972) 'Parental investment and sexual selection', in B. Campbell (ed.) Sexual selection and the descent of man, 1871-1971. Aldine-Atherton. Chicago, Illinois.

Truman, J. W., \& Riddiford, L. M. (1999). The origins of insect metamorphosis. Nature, 401, 447-452.

van den Berghe, E. P. (1990). On the habits and habitat of Omus (Coleoptera: Cicindelae). Cicindela 22, 61-68

van den Berghe, E. P. (1994). Omus cazieri, a new species from southern Oregon (Coleoptera: Cicindelae). Cicindela 26, 61-68.

Van Valen, L. M. (1973). A new evolutionary law. Evolutionary Theory, 1, 1-30. 
Vandermeer, J. H. (1972). Niche Theory. Annual Review of Ecology and Systematics, 3, $107-132$

Vie, J.C., Hilton-Taylor, C. and Stuart, S.N. (eds) (2009) Wildlife in a Changing World - An analysis of the 2008 IUCN Red List of Threatened Species. IUCN, Gland, Switzerland.

Vogler, A. P. \& Pearson, D. L. (1996). A molecular phylogeny of the tiger beetles (Cicindelidae): congruence of mitochondrial and nuclear rDNA data sets. Molecular Phylogenetics and Evolution, 6(3), 321-338.

Vogler, A. P. \& Welsh, A. (1997). Phylogeny of North American Cicindela tiger beetles inferred from multiple mitochondrial DNA sequences. Molecular Phylogenetics and Evolution, 8(2), 225-235.

Vogler, A. P., Cardoso, A., \& Barraclough, T. G. (2005). Exploring rate variation among and within sites in a densely sampled tree: species level phylogenetics of north american tiger beetles (genus Cicindela). Systematic Biology, 54(1), 4-20.

Wainwright, P. C. \& Reilly, S. M. (1994). Ecological morphology: integrative organismal biology. University of Chicago Press, Chicago, IL.

Ward, A. B., Weigl, P. D., \& Conroy, R. M. (2002). Functional morphology of raptor hindlimbs: implications for resource partitioning. Auk, 119(4), 1052-1063.

Watson, P. J., Arnqvist, G. \& Stallmann, R. R. (1998). Sexual conflict and the energetic costs of mating and mate choice in water striders. American Naturalist, 151, 46-58.

Weckerly, F. W. (1998). Sexual-size dimorphism: influence of mass and mating systems in the most dimorphic mammals. Journal of Mammalogy, 33-52.

Welch, B. L. (1947). The generalization of 'Student's' problem when several different population variances are involved. Biometrika, 34, 28-35

Wilcox, R. S. (1984). Male Copulatory Guarding Enhances Female Foraging in a Water Strider. Behavioral Ecology and Sociobiology, 15, 171-174.

Willis, H. L. (1967). Bionomics and zoogeography of tiger beetles of saline habitats in the central United States (Coleoptera: Cincindelidae). Kansas University Science Bulletin, 48, 145-313.

Wilson, D. S. (1975). The adequacy of body size as a niche difference. American Naturalist, 109, 769-784.

Zacharuk, R. Y. (1980). Ultrastructure and Function of Insect Chemosensilla. Annual Review of Entomology, 25, 27-47.

Zelditch, M.L., D.L. Swiderski, H.D. Sheets and W.L. Fink. (2004). Geometric morphometrics for biologists: A primer. Elsevier Academic Press, San Diego, California.

Zuk, M. \& Kolluru, G. R. (1998). Exploitation of Sexual Signals by Predators and Parasitoids. Quarterly Review of Biology, 73, 415-438. 


\section{Appendix A: Gross morphometric data as CSV file.}

$\mathrm{SPSX}=$ species $\&$ sex combined, $\mathrm{SP}=$ species $(\mathrm{A}=O$. audouini, $\mathrm{D}=O$. dejeanii $), \mathrm{SX}=$ sex $(\mathrm{F}=$ female, $\mathrm{M}=$ male $), \mathrm{ID}=$ unique specimen $\mathrm{ID}$ code, $\mathrm{YEAR}=$ year of capture. All other header abbreviations are given in Table 2.4 .

SPSX , SP , SX , ID , YR , BL , AN , WS , PL , PW , EL , EW, TF , TI , MN , HW

$\mathrm{AF}, \mathrm{A}, \mathrm{F}, 25.09,2009,16.4,9.1,3.2,3.65,4.35,9.5,6.1,5.1,4.8,2.8,3.85$

$\mathrm{AF}, \mathrm{A}, \mathrm{F}, 26.09,2009,15.6,8.8,3.15,3.75,4.3,9.75,6,5.2,5.2,2.8,3.85$

$\mathrm{AF}, \mathrm{A}, \mathrm{F}, 27.09,2009,16.1,8.6,3.05,3.5,4.15,8.8,6,5.1,4.6,2.65,3.8$

$\mathrm{AF}, \mathrm{A}, \mathrm{F}, 28.09,2009,16.3,8.6,3.2,3.55,4.1,9.6,6.25,4.8,4.6,2.8,3.85$

$\mathrm{AF}, \mathrm{A}, \mathrm{F}, 29.09,2009,16.6,8.8,3.15,3.65,4.15,9.7,6.1,5.1,4.9,2.9,3.9$

$\mathrm{AF}, \mathrm{A}, \mathrm{F}, 30.09,2009,16.4,8.9,3.4,4.05,4.5,9.6,6.3,5.3,5,2.85,4.05$

$\mathrm{AF}, \mathrm{A}, \mathrm{F}, 31.09,2009,16.4,9.3,3.2,3.75,4.5,9.8,6.25,5.3,5.2,2.9,4.05$

$\mathrm{AF}, \mathrm{A}, \mathrm{F}, 36.09,2009,15.9,8.5,3.2,3.55,4.25,9.25,6,5,5,2.75,3.8$

$\mathrm{AF}, \mathrm{A}, \mathrm{F}, 37.09,2009,15,8.3,3.1,3.6,4.15,9.3,5.8,4.8,4.8,2.7,3.75$

$\mathrm{AF}, \mathrm{A}, \mathrm{F}, 39.09,2009,15.4,8.4,3.1,3.75,4.3,9,5.7,4.9,4.7,2.65,3.85$

$\mathrm{AF}, \mathrm{A}, \mathrm{F}, 40.09,2009,15.4,8.4,3.2,3.7,4.3,8.95,6.1,5.1,5,2.75,3.95$

$\mathrm{AF}, \mathrm{A}, \mathrm{F}, 41.09,2009,16.1,8.7,3.2,3.75,4.3,9.3,6.1,5,4.9,2.8,3.9$

$\mathrm{AF}, \mathrm{A}, \mathrm{F}, 42.09,2009,16,8.7,3.15,3.65,4.4,9.05,6,4.8,4.8,2.75,3.9$

$\mathrm{AF}, \mathrm{A}, \mathrm{F}, 43.09,2009,15.3,8.1,3,3.45,4.15,8.85,5.75,4.8,4.8,2.6,3.7$

$\mathrm{AF}, \mathrm{A}, \mathrm{F}, 44.09,2009,15.2,8.4,3.25,3.55,4.3,9.1,5.6,5,4.8,2.69,3.9$

$\mathrm{AF}, \mathrm{A}, \mathrm{F}, 45.09,2009,15.4,8.1,3.05,3.5,4.15,8.9,5.65,4.8,4.6,2.74,3.7$

$\mathrm{AF}, \mathrm{A}, \mathrm{F}, 47.09,2009,16.2,8.6,3.15,3.7,4.25,9.6,6.2,5.1,5,2.9,3.95$

$\mathrm{AF}, \mathrm{A}, \mathrm{F}, 48.09,2009,15.7,8.2,3.1,3.65,4.3,9.15,5.8,4.9,4.7,2.67,3.9$

$\mathrm{AF}, \mathrm{A}, \mathrm{F}, 49.09,2009,16.6,8.5,3,3.65,4.15,9.25,6,5,4.6,2.75,3.85$

$\mathrm{AF}, \mathrm{A}, \mathrm{F}, 50.09,2009,15.6,8.1,3.1,3.65,4.3,9.3,6,4.9,5,2.75,3.75$

$\mathrm{AF}, \mathrm{A}, \mathrm{F}, 51.09,2009,15.9,8.7,3.25,3.75,4.55,9.85,6.2,5.1,5.1,2.8,3.95$

$\mathrm{AF}, \mathrm{A}, \mathrm{F}, 52.09,2009,16.2,9.4,3.25,3.75,4.4,9.7,6.25,5.1,4.8,2.85,3.95$

$\mathrm{AF}, \mathrm{A}, \mathrm{F}, 53.09,2009,16.9,8.9,3.3,3.75,4.55,9.8,6.15,5.3,5,2.85,3.95$

$\mathrm{AF}, \mathrm{A}, \mathrm{F}, 54.09,2009,16,8.7,3.2,3.7,4.35,9.6,6.1,5.1,5.1,2.85,4$

$\mathrm{AF}, \mathrm{A}, \mathrm{F}, 57.09,2009,16,8.8,3,3.4,4.3,9.1,5.8,5,4.8,2.8,3.75$

$\mathrm{AF}, \mathrm{A}, \mathrm{F}, 64.09,2009,15.7,8.8,3.15,3.65,4.4,9.05,5.85,5.1,4.9,2.75,3.85$

$\mathrm{AF}, \mathrm{A}, \mathrm{F}, 65.09,2009,16.7,8.5,3.15,3.8,4.4,9.4,5.95,5.1,4.9,2.75,3.9$

$\mathrm{AF}, \mathrm{A}, \mathrm{F}, 67.09,2009,15.6,8.2,3.25,3.6,4.4,9.2,6,4.9,4.9,2.8,3.9$

$\mathrm{AF}, \mathrm{A}, \mathrm{F}, 68.09,2009,14.9,7.9,2.95,3.35,4.1,8.9,5.55,4.6,4.7,2.6,3.65$

$\mathrm{AF}, \mathrm{A}, \mathrm{F}, 71.09,2009,15.5,8.3,3.1,3.55,4.15,9.1,5.75,4.8,4.8,2.7,3.8$

$\mathrm{AF}, \mathrm{A}, \mathrm{F}, 74.09,2009,15,7.9,3,3.6,4.15,8.7,5.65,4.6,4.6,2.65,3.65$

$\mathrm{AF}, \mathrm{A}, \mathrm{F}, 76.09,2009,16.4,8.8,3.1,3.7,4.25,9.2,6,5,5.2,2.95,3.9$

$\mathrm{AF}, \mathrm{A}, \mathrm{F}, 77.09,2009,16.2,8.5,3.15,3.55,4.25,9.55,6.05,5,4.8,2.74,3.8$

$\mathrm{AF}, \mathrm{A}, \mathrm{F}, 79.09,2009,15.9,8.7,3.15,3.7,4.35,9.3,6.05,4.9,4.9,2.85,3.85$

$\mathrm{AF}, \mathrm{A}, \mathrm{F}, 80.09,2009,15.9,8.6,3.35,3.8,4.45,9.35,6.1,5,5.1,2.8,3.9$

$\mathrm{AF}, \mathrm{A}, \mathrm{F}, 84.09,2009,16.4,8.6,3.15,3.7,4.3,9.25,6,4.8,4.7,2.8,3.8$

$\mathrm{AF}, \mathrm{A}, \mathrm{F}, 109.09,2009,16.4,8.8,3.3,3.95,4.55,9.8,6.3,5.1,4.9,2.9,4$

$\mathrm{AF}, \mathrm{A}, \mathrm{F}, 112.09,2009,14,7.8,2.95,3.55,4,8.35,5.4,4.6,4.3,2.65,3.6$

$\mathrm{AF}, \mathrm{A}, \mathrm{F}, 113.09,2009,16,8.7,3.25,3.8,4.3,9.45,6.25,5.1,5.1,2.8,3.9$

$\mathrm{AF}, \mathrm{A}, \mathrm{F}, 114.09,2009,15.8,8,3.15,3.5,4.35,9.2,6,5.1,4.8,2.7,3.95$

$\mathrm{AF}, \mathrm{A}, \mathrm{F}, 116.09,2009,16.9,8.6,3.15,3.7,4.25,9.1,6,4.8,4.8,2.7,3.85$

$\mathrm{AF}, \mathrm{A}, \mathrm{F}, 117.09,2009,15.7,8.5,3.1,3.55,4.3,9.1,5.8,4.9,4.9,2.7,3.75$

$\mathrm{AF}, \mathrm{A}, \mathrm{F}, 118.09,2009,15.3,8.6,3.1,3.55,4.15,8.9,5.8,5,5.2,2.7,3.8$

$\mathrm{AF}, \mathrm{A}, \mathrm{F}, 120.09,2009,15.9,8.8,3.15,3.7,4.3,9.3,5.95,5.1,5.2,2.85,3.85$

$\mathrm{AF}, \mathrm{A}, \mathrm{F}, 123.09,2009,15.4,8.3,3.3,3.6,4.25,9.2,5.8,5,5.1,2.8,3.75$

$\mathrm{AF}, \mathrm{A}, \mathrm{F}, 124.09,2009,14.8,8.1,2.9,3.4,4,8.7,5.55,4.7,4.7,2.8,3.6$

$\mathrm{AF}, \mathrm{A}, \mathrm{F}, 126.09,2009,16.5,8.8,3.2,3.7,4.45,9.8,6.2,5.3,5,2.9,3.85$

$\mathrm{AF}, \mathrm{A}, \mathrm{F}, 132.09,2009,16.5,8.5,3.15,3.6,4.45,9.1,5.85,5.2,4.9,2.75,3.8$

$\mathrm{AF}, \mathrm{A}, \mathrm{F}, 133.09,2009,16,8.9,3.25,3.6,4.4,9.35,6.15,5,5,2.85,3.4$ 
$\mathrm{AF}, \mathrm{A}, \mathrm{F}, 135.09,2009,16.2,9.2,3.5,3.8,4.55,9.6,6.45,5.2,5.1,2.65,4$ $\mathrm{AF}, \mathrm{A}, \mathrm{F}, 138.09,2009,16.4,8.5,3.4,3.7,4.4,9.9,6.25,5.1,5.1,2.85,3.9$ $A F, A, F, 139.09,2009,16,9.2,3.2,3.55,4.15,9.6,6.15,5.1,5.1,2.8,3.9$ $\mathrm{AF}, \mathrm{A}, \mathrm{F}, 140.09,2009,16.6,8.7,3.3,3.8,4.5,9.75,6.3,5.3,5.3,2.9,4$ $\mathrm{AF}, \mathrm{A}, \mathrm{F}, 141.09,2009,16.1,8.2,3.3,3.65,4.5,9.8,6.2,5,4.7,2.8,3.9$

$\mathrm{AF}, \mathrm{A}, \mathrm{F}, 144.09,2009,16.2,8.6,3.3,3.75,4.3,9.65,6.3,5.1,4.9,2.75,3.95$ $\mathrm{AF}, \mathrm{A}, \mathrm{F}, 145.09,2009,15,7.9,3,3.35,4.05,8.65,5.3,4.5,4.5,2.6,3.6$ $\mathrm{AF}, \mathrm{A}, \mathrm{F}, 5.1,2010,15.2,8.4,2.9,3.55,3.75,8.2,5.15,4.6,4.5,3.15,3.55$ $\mathrm{AF}, \mathrm{A}, \mathrm{F}, 21.1,2010,17.2,9.2,3.2,3.75,4.45,9.55,6.1,5,4.7,2.95,3.9$ $\mathrm{AF}, \mathrm{A}, \mathrm{F}, 22.1,2010,17.1,8.7,3.25,3.85,4.45,9.55,6.05,5.2,5.1,3,3.9$ $\mathrm{AF}, \mathrm{A}, \mathrm{F}, 23.1,2010,16.7,8.6,3.25,3.7,4.45,9.2,6.15,5.1,5.1,2.9,3.95$ $\mathrm{AF}, \mathrm{A}, \mathrm{F}, 24.1,2010,17.5,9,3.35,3.85,4.55,10,3.35,5.1,4.9,2.95,4.15$ $\mathrm{AF}, \mathrm{A}, \mathrm{F}, 25.1,2010,15.7,8.2,3.1,3.5,4.2,8.95,5.85,4.9,4.8,2.85,3.75$ $\mathrm{AF}, \mathrm{A}, \mathrm{F}, 27.1,2010,16.5,8.7,3.15,3.75,4.3,9.65,6.25,5.1,5,2.8,4$ $\mathrm{AF}, \mathrm{A}, \mathrm{F}, 28.1,2010,15,8.1,3,3.6,4.05,8.95,5.8,4.9,4.7,2.65,3.75$ $\mathrm{AF}, \mathrm{A}, \mathrm{F}, 29.1,2010,16.4,8.8,3.1,3.7,4.35,9.55,6.1,5.1,5.1,2.8,3.9$ $\mathrm{AF}, \mathrm{A}, \mathrm{F}, 35.1,2010,16.4,8.8,3.1,3.65,4.3,9.3,5.95,5.1,4.9,2.9,3.9$ $\mathrm{AF}, \mathrm{A}, \mathrm{F}, 100.1,2010,16,9.2,3.25,3.65,4.35,9.35,6.1,5.1,5,2.85,4.05$ $\mathrm{AF}, \mathrm{A}, \mathrm{F}, 101.1,2010,17.3,8.7,3.2,3.65,4.3,9.35,6.2,5.1,4.9,2.95,4.05$ $\mathrm{AF}, \mathrm{A}, \mathrm{F}, 102.1,2010,16.7,8.4,3.1,3.6,4.1,9.1,5.8,4.9,4.9,2.75,3.7$ $\mathrm{AF}, \mathrm{A}, \mathrm{F}, 103.1,2010,17,9,3.1,3.65,4.35,9.15,6.1,5.1,5.1,2.8,3.9$ $A M, A, M, 32.09,2009,14.7,8.5,3.05,3.7,4,8.85,5.6,4.4,4.7,3.05,3.65$ $\mathrm{AM}, \mathrm{A}, \mathrm{M}, 33.09,2009,15.8,8.8,3,3.65,4.05,8.75,5.55,4.9,4.9,3.05,3.65$ $\mathrm{AM}, \mathrm{A}, \mathrm{M}, 34.09,2009,15.3,8.6,3.05,3.6,4,8.75,5.6,4.7,4.7,3.05,3.7$ $\mathrm{AM}, \mathrm{A}, \mathrm{M}, 55.09,2009,15,8.5,2.95,3.6,3.95,8.75,5.2,4.9,4.7,3.13,3.75$ $\mathrm{AM}, \mathrm{A}, \mathrm{M}, 56.09,2009,14.5,8.7,2.9,3.35,3.8,8.6,5.15,4.8,4.8,3.04,3.6$ $\mathrm{AM}, \mathrm{A}, \mathrm{M}, 58.09,2009,15.3,8.9,2.8,3.55,3.9,8.55,5.3,4.6,4.9,3.05,3.55$ $\mathrm{AM}, \mathrm{A}, \mathrm{M}, 59.09,2009,15.7,9.4,3.15,3.75,4.25,9.05,5.9,5,5,3.2,3.9$ $A M, A, M, 61.09,2009,14.6,8.5,2.75,3.45,3.8,8.6,5.3,4.7,4.8,3.05,3.55$ $A M, A, M, 62.09,2009,14.9,8.7,3,3.6,4.05,8.45,5.4,4.9,5,3.1,3.65$ $\mathrm{AM}, \mathrm{A}, \mathrm{M}, 63.09,2009,14.4,8.7,2.95,3.5,3.8,8.4,5.1,4.7,4.9,2.95,3.55$ $\mathrm{AM}, \mathrm{A}, \mathrm{M}, 69.09,2009,14.2,7.9,2.85,3.55,3.75,8.3,5.1,4.6,4.6,2.95,3.45$ $A M, A, M, 70.09,2009,14.7,9,2.95,3.65,4.05,8.8,5.3,4.9,4.9,3.1,3.7$ $\mathrm{AM}, \mathrm{A}, \mathrm{M}, 72.09,2009,14.9,8.2,3,3.45,3.9,8.3,5.2,4.6,4.7,3.1,3.55$ $A M, A, M, 73.09,2009,14.8,8.3,3,3.7,4.05,8.45,5.45,4.7,4.9,3.2,3.65$ $\mathrm{AM}, \mathrm{A}, \mathrm{M}, 75.09,2009,14.2,8.2,2.85,3.6,3.85,8.05,5.05,4.8,4.7,3.1,3.6$ $A M, A, M, 78.09,2009,15.1,8.8,3,3.7,4.15,9.05,5.65,5,5,3.1,3.65$ $\mathrm{AM}, \mathrm{A}, \mathrm{M}, 110.09,2009,15.7,9.1,3.05,3.65,4.1,9.1,5.5,4.9,4.8,3.13,3.75$ $A M, A, M, 111.09,2009,15.4,8.7,2.95,3.65,4,8.95,5.5,5,4.6,3.21,3.7$ $\mathrm{AM}, \mathrm{A}, \mathrm{M}, 115.09,2009,14.8,8.8,3,3.6,3.95,8.95,5.45,4.8,4.7,3.15,3.65$ $\mathrm{AM}, \mathrm{A}, \mathrm{M}, 119.09,2009,14,8.5,2.9,3.35,3.9,8.95,5.15,4.8,4.8,3.04,3.6$ $\mathrm{AM}, \mathrm{A}, \mathrm{M}, 121.09,2009,14.4,9,3.1,3.55,4.2,9,5.65,5,5.2,3.1,3.7$ $\mathrm{AM}, \mathrm{A}, \mathrm{M}, 122.09,2009,13.8,8.4,2.75,3.3,3.85,8.15,5.25,4.6,4.8,3.05,3.55$ $A M, A, M, 125.09,2009,15,8.9,2.95,3.6,3.95,8.55,5.35,4.7,4.8,3.15,3.65$ $\mathrm{AM}, \mathrm{A}, \mathrm{M}, 127.09,2009,14.3,8.4,2.85,3.55,3.85,8.55,5.05,4.7,4.9,2.9,3.5$ $\mathrm{AM}, \mathrm{A}, \mathrm{M}, 128.09,2009,13.9,8.4,2.9,3.05,3.7,8.55,5.1,4.6,4.5,3.05,3.5$ $\mathrm{AM}, \mathrm{A}, \mathrm{M}, 129.09,2009,14.8,8.6,2.9,3.65,4,8.75,5.35,4.7,4.9,3.2,3.65$ $A M, A, M, 130.09,2009,14.7,9.1,3,3.55,4,8.7,5.55,4.9,5.2,3,3.7$ $\mathrm{AM}, \mathrm{A}, \mathrm{M}, 131.09,2009,14,8.4,2.85,3.4,3.8,8.25,4.8,4.6,4.7,3.1,3.45$ $\mathrm{AM}, \mathrm{A}, \mathrm{M}, 134.09,2009,14.4,9,2.8,3.45,3.7,8.15,5.15,4.6,4.4,3,3.5$ $\mathrm{AM}, \mathrm{A}, \mathrm{M}, 136.09,2009,13.5,8.7,2.75,3.25,3.55,8.55,5.2,4.7,4.8,3,3.4$ $\mathrm{AM}, \mathrm{A}, \mathrm{M}, 137.09,2009,14.9,8.7,3.1,3.5,4.1,8.8,5.6,4.9,4.9,3.3,3.75$ $\mathrm{AM}, \mathrm{A}, \mathrm{M}, 142.09,2009,13.4,8.5,2.8,3.45,3.85,8.25,5.1,4.7,4.6,2.95,3.6$ $\mathrm{AM}, \mathrm{A}, \mathrm{M}, 143.09,2009,13.6,8.1,2.9,3.55,3.85,8.55,5.1,4.6,4.4,3,3.5$ $\mathrm{AM}, \mathrm{A}, \mathrm{M}, 146.09,2009,14.9,8.4,3.1,3.6,4.05,9,5.7,5,5.1,3.15,3.7$ $\mathrm{AM}, \mathrm{A}, \mathrm{M}, 147.09,2009,14.6,8.7,3,3.55,3.95,8.65,5.4,4.9,4.7,3.1,3.5$ $\mathrm{AM}, \mathrm{A}, \mathrm{M}, 4.1,2010,15.8,9,3,3.6,4.1,8.65,5.5,4.8,4.8,3.15,3.7$ 
$\mathrm{AM}, \mathrm{A}, \mathrm{M}, 15.1,2010,14,8.2,2.9,3.45,4,8.25,5.3,4.5,4.3,3.15,3.5$

$\mathrm{AM}, \mathrm{A}, \mathrm{M}, 16.1,2010,15.5,8.9,3.1,3.7,3.95,8.9,5.7,5,4.9,3.35,3.7$

$\mathrm{AM}, \mathrm{A}, \mathrm{M}, 17.1,2010,14.4,8.4,2.75,3.4,3.75,7.95,5,4.4,4.5,2.95,3.4$

$\mathrm{AM}, \mathrm{A}, \mathrm{M}, 18.1,2010,15.7,8.9,3,3.65,4,8.95,5.8,5.1,5.1,3.2,3.65$

$\mathrm{AM}, \mathrm{A}, \mathrm{M}, 19.1,2010,15.2,9.1,3,3.55,4,8.15,5.55,4.7,4.7,3.05,3.6$

$\mathrm{AM}, \mathrm{A}, \mathrm{M}, 20.1,2010,14.9,9.1,3,3.65,4.1,8.65,5.6,4.9,5,3.1,3.75$

$\mathrm{AM}, \mathrm{A}, \mathrm{M}, 30.1,2010,15.7,9,3,3.55,3.85,8.45,5.4,4.9,4.9,3,3.6$

$\mathrm{AM}, \mathrm{A}, \mathrm{M}, 32.1,2010,14.5,8.8,3,3.45,4,8.3,5.15,4.6,4.8,3.1,3.6$

$\mathrm{AM}, \mathrm{A}, \mathrm{M}, 40.1,2010,15.2,9,2.95,3.6,4.05,8.7,5.5,4.9,4.9,3.2,3.7$

$\mathrm{AM}, \mathrm{A}, \mathrm{M}, 45.1,2010,14.6,8.8,2.9,3.55,4,8.25,5.3,4.8,4.8,3.1,3.6$

$\mathrm{AM}, \mathrm{A}, \mathrm{M}, 46.1,2010,15.4,9.2,3,3.8,4.25,8.8,5.65,4.9,5.1,3.25,3.85$

$\mathrm{AM}, \mathrm{A}, \mathrm{M}, 53.1,2010,14.9,8.7,2.85,3.45,3.95,8.5,5.3,4.8,4.8,3.05,3.65$

$\mathrm{AM}, \mathrm{A}, \mathrm{M}, 54.1,2010,14.4,8.1,2.65,3.1,3.7,8.15,5,4.4,4.4,3.1,3.4$

$\mathrm{AM}, \mathrm{A}, \mathrm{M}, 62.1,2010,16.1,9.4,3.15,3.8,4.2,9.15,5.4,5.2,5.2,3.2,3.95$

$\mathrm{AM}, \mathrm{A}, \mathrm{M}, 63.1,2010,15.5,8.2,2.85,3.35,3.8,8.25,5.1,4.5,4.6,3.15,3.55$

$\mathrm{AM}, \mathrm{A}, \mathrm{M}, 64.1,2010,14.2,8.8,2.8,3.25,3.75,8.1,5.1,4.7,4.7,3.1,3.4$

$\mathrm{AM}, \mathrm{A}, \mathrm{M}, 65.1,2010,14.7,8.2,2.75,3.5,3.85,8.5,5.25,4.6,4.8,3.1,3.55$

$\mathrm{AM}, \mathrm{A}, \mathrm{M}, 66.1,2010,14.7,8.6,2.7,3.35,3.8,8,5.1,4.7,4.7,3.1,3.45$

$\mathrm{AM}, \mathrm{A}, \mathrm{M}, 67.1,2010,15.2,8.5,2.75,3.5,3.85,8.25,5.25,4.7,4.8,3.05,3.55$

$\mathrm{AM}, \mathrm{A}, \mathrm{M}, 68.1,2010,15.5,9,3,3.75,4.2,8.95,5.65,5,5,3.2,3.8$

$\mathrm{AM}, \mathrm{A}, \mathrm{M}, 69.1,2010,15.1,9,2.95,3.6,4.1,8.6,5.45,4.9,4.8,3.2,3.7$

$\mathrm{AM}, \mathrm{A}, \mathrm{M}, 70.1,2010,14.9,8.8,2.9,3.45,3.9,8.6,5.4,4.8,4.8,3.25,3.65$

$\mathrm{AM}, \mathrm{A}, \mathrm{M}, 71.1,2010,14.6,8.4,2.95,3.5,4,8.25,5.3,4.7,4.7,3.1,3.55$

$\mathrm{AM}, \mathrm{A}, \mathrm{M}, 79.1,2010,16.1,9.4,2.9,3.7,3.95,9.25,5.4,5,5.1,3.2,3.6$

$\mathrm{AM}, \mathrm{A}, \mathrm{M}, 80.1,2010,15.2,8.5,2.85,3.5,4.05,8.75,5.45,4.7,4.7,3.15,3.6$

$\mathrm{AM}, \mathrm{A}, \mathrm{M}, 81.1,2010,14.8,8.6,2.75,3.45,3.9,8.1,5.25,4.6,4.6,2.95,3.5$

$\mathrm{AM}, \mathrm{A}, \mathrm{M}, 85.1,2010,15.2,8.6,2.95,3.5,4.05,8.7,5.45,4.8,4.6,3.15,3.65$

$\mathrm{AM}, \mathrm{A}, \mathrm{M}, 86.1,2010,14.5,8.7,2.8,3.5,3.95,8,5.3,4.7,4.8,3.05,3.55$

$\mathrm{AM}, \mathrm{A}, \mathrm{M}, 105.1,2010,14.6,8.9,2.8,3.35,3.8,8.55,5.45,4.8,4.8,3.15,3.55$

$\mathrm{AM}, \mathrm{A}, \mathrm{M}, 116.1,2010,15.3,8.6,2.75,3.3,3.85,8.1,5.15,4.6,4.6,3.15,3.5$

$\mathrm{AM}, \mathrm{A}, \mathrm{M}, 117.1,2010,15.3,9.1,3,3.5,3.95,8.65,5.15,4.9,4.7,3.2,3.6$

$\mathrm{AM}, \mathrm{A}, \mathrm{M}, 118.1,2010,15,8.7,2.95,3.5,3.9,8.5,5.25,4.7,4.7,3.1,3.6$

$\mathrm{AM}, \mathrm{A}, \mathrm{M}, 120.1,2010,15.6,9.1,3,3.65,4.05,8.95,5.55,4.9,4.7,3.2,3.7$

$\mathrm{AM}, \mathrm{A}, \mathrm{M}, 129.1,2010,15.2,8.8,2.8,3.5,3.7,8.3,5.15,4.7,4.6,3.05,3.55$

$\mathrm{DF}, \mathrm{D}, \mathrm{F}, 81.09,2009,21.2,10.7,3.8,4.45,6.6,11.25,8,6.4,6.4,4.29,5.25$

$\mathrm{DF}, \mathrm{D}, \mathrm{F}, 85.09,2009,19.4,11.6,3.7,4.3,6.25,11.15,7.5,6.1,6.1,4.18,4.9$

$\mathrm{DF}, \mathrm{D}, \mathrm{F}, 87.09,2009,18.8,10.1,3.6,4.25,6.25,10.35,7.55,5.8,5.8,4.08,4.85$

$\mathrm{DF}, \mathrm{D}, \mathrm{F}, 88.09,2009,20.5,10.9,3.5,4.7,6.7,11.65,8,6.3,6.4,4.35,5.3$

$\mathrm{DF}, \mathrm{D}, \mathrm{F}, 91.09,2009,21.2,11,3.9,4.6,6.6,11.5,8.05,6.5,6.7,4.45,5.35$

$\mathrm{DF}, \mathrm{D}, \mathrm{F}, 92.09,2009,20.5,11.3,3.85,4.55,6.45,11.55,8.05,6.4,6.3,4.3,5.2$

$\mathrm{DF}, \mathrm{D}, \mathrm{F}, 93.09,2009,21.9,10.8,3.9,4.55,6.5,11.6,8.1,6.3,6.3,4.41,5.3$

$\mathrm{DF}, \mathrm{D}, \mathrm{F}, 94.09,2009,20,10.4,3.8,4.5,6.6,11.5,7.9,6,6,4.2,5.3$

$\mathrm{DF}, \mathrm{D}, \mathrm{F}, 95.09,2009,20.7,10.3,3.85,4.5,6.7,11.95,8.1,6.4,6.6,4.06,5.35$

$\mathrm{DF}, \mathrm{D}, \mathrm{F}, 96.09,2009,20.2,10.5,3.75,4.45,6.4,11.75,8,6.4,6.4,4.35,5.3$

$\mathrm{DF}, \mathrm{D}, \mathrm{F}, 100.09,2009,21,10.9,3.9,4.6,6.9,12.15,8.1,6.6,6.7,4.36,5.4$

$\mathrm{DF}, \mathrm{D}, \mathrm{F}, 103.09,2009,21.2,11.2,4.1,4.6,6.7,12.15,8.45,6.5,6.6,4.54,5.45$

$\mathrm{DF}, \mathrm{D}, \mathrm{F}, 105.09,2009,20.3,10.7,3.9,4.55,6.45,11.6,8.05,6.4,6.5,4.45,5.3$

$\mathrm{DF}, \mathrm{D}, \mathrm{F}, 107.09,2009,18.6,10.4,3.7,4.25,6.1,11.15,7.7,6.1,6.2,4.13,5.05$

$\mathrm{DF}, \mathrm{D}, \mathrm{F}, 108.09,2009,20.5,11.1,4,4.55,6.65,11.7,8.05,6.4,6.4,4.27,5.45$

$\mathrm{DF}, \mathrm{D}, \mathrm{F}, 251.09,2009,20,10.4,3.7,4.45,6.25,11.35,7.65,6.2,6.3,4.3,5.15$

$\mathrm{DF}, \mathrm{D}, \mathrm{F}, 252.09,2009,20.3,11.3,3.85,4.7,6.45,11.3,7.65,6.4,6.4,4.35,5.25$

$\mathrm{DF}, \mathrm{D}, \mathrm{F}, 3.1,2010,20.6,11.2,3.95,4.6,6.75,11.2,8.1,6.4,6.8,4.3,5.4$

$\mathrm{DF}, \mathrm{D}, \mathrm{F}, 7.1,2010,20.8,11.1,4,4.6,6.75,12.2,8.2,6.4,6.5,4.7,5.4$

$\mathrm{DF}, \mathrm{D}, \mathrm{F}, 8.1,2010,21.2,10.8,4,4.65,6.8,11.65,8.2,6.5,6.5,4.5,5.5$

$\mathrm{DF}, \mathrm{D}, \mathrm{F}, 9.1,2010,21.3,10.8,3.85,4.45,6.65,11.5,8.2,6.2,6.5,4.35,5.25$

$\mathrm{DF}, \mathrm{D}, \mathrm{F}, 26.1,2010,20.1,10.9,3.85,4.6,6.55,11.55,8.25,6.2,6.3,4.45,5.3$

$\mathrm{DF}, \mathrm{D}, \mathrm{F}, 33.1,2010,19.8,10.4,3.75,4.05,6.45,11.3,8.1,6.1,6.4,4.3,5.15$ 
$\mathrm{DF}, \mathrm{D}, \mathrm{F}, 34.1,2010,20,10.2,3.8,4.5,6.5,11.2,8,6.1,6.1,4.35,5.1$ $\mathrm{DF}, \mathrm{D}, \mathrm{F}, 47.1,2010,20.3,10.5,3.65,4.35,6.15,11.05,7.55,6,6,4.2,5.05$ $\mathrm{DF}, \mathrm{D}, \mathrm{F}, 49.1,2010,19.9,10.6,3.8,4.5,6.4,11.1,7.95,6.2,6.2,4.25,5.15$ $\mathrm{DF}, \mathrm{D}, \mathrm{F}, 50.1,2010,19.2,10.2,3.5,4.3,6.15,10.65,7.75,5.8,6.1,4.1,4.9$ $\mathrm{DF}, \mathrm{D}, \mathrm{F}, 72.1,2010,21,10.9,3.9,4.7,6.6,12,8.35,6.4,6.7,4.35,5.35$ $\mathrm{DF}, \mathrm{D}, \mathrm{F}, 87.1,2010,19.6,10.5,3.75,4.25,6.4,11.05,7.7,6.1,6.3,4.15,5.05$ $\mathrm{DF}, \mathrm{D}, \mathrm{F}, 88.1,2010,20,11,3.8,4.65,6.65,11.3,7.95,6.3,6.5,4.3,5.3$ $\mathrm{DF}, \mathrm{D}, \mathrm{F}, 90.1,2010,20.5,10.6,3.75,4.5,6.5,11.1,7.8,6.2,6.4,4.3,5.25$ $\mathrm{DF}, \mathrm{D}, \mathrm{F}, 125.1,2010,20.5,10.6,3.75,4.55,6.55,11.7,8,6.4,6.4,4.35,5.25$ $\mathrm{DF}, \mathrm{D}, \mathrm{F}, 126.1,2010,20.2,10.8,3.8,4.5,6.4,11.3,8.1,6.3,6.5,4.35,5.25$ $\mathrm{DF}, \mathrm{D}, \mathrm{F}, 133.1,2010,21.2,11,3.85,4.6,6.65,11.9,8.05,6.2,6.2,4.3,5.35$ $\mathrm{DF}, \mathrm{D}, \mathrm{F}, 137.1,2010,21,11,3.7,4.45,6.4,10.95,7.75,6.1,6.1,4.3,5.15$ $\mathrm{DF}, \mathrm{D}, \mathrm{F}, 139.1,2010,20.3,11.1,3.7,4.3,6.3,11.35,8.05,6.3,6.5,4.4,5.15$ $\mathrm{DF}, \mathrm{D}, \mathrm{F}, 145.1,2010,20.2,10.4,3.8,4.5,6.25,10.95,7.8,6.2,6.1,4.25,5.1$ $\mathrm{DF}, \mathrm{D}, \mathrm{F}, 146.1,2010,21.2,10.9,3.75,4.6,6.35,11.6,7.9,6.4,6.4,4.45,5.15$ $\mathrm{DF}, \mathrm{D}, \mathrm{F}, 150.1,2010,20.5,11.2,4,4.55,6.7,11.45,8.2,6.5,6.5,4.45,5.4$ $\mathrm{DF}, \mathrm{D}, \mathrm{F}, 151.1,2010,20.2,11.3,4,4.55,6.8,11.75,8.05,6.4,6.4,4.45,5.35$ $\mathrm{DF}, \mathrm{D}, \mathrm{F}, 153.1,2010,19.6,11,3.9,4.45,6.55,11.15,7.75,6.4,6.4,4.3,5.15$ $\mathrm{DF}, \mathrm{D}, \mathrm{F}, 154.1,2010,19.4,11.1,3.8,4.3,6.35,11.3,7.85,6.3,6.3,4.1,5$ $\mathrm{DF}, \mathrm{D}, \mathrm{F}, 156.1,2010,20.7,11.4,4.05,4.6,6.6,11.8,8.3,6.5,6.5,4.3,5.4$ $\mathrm{DM}, \mathrm{D}, \mathrm{M}, 82.09,2009,19.5,11,3.7,4.6,6.2,11.1,7.45,6.1,6.4,4.51,4.95$ $\mathrm{DM}, \mathrm{D}, \mathrm{M}, 83.09,2009,18.1,11.4,3.5,4.25,5.65,10.6,7.25,5.7,6.1,4.17,4.65$ $\mathrm{DM}, \mathrm{D}, \mathrm{M}, 86.09,2009,19,11.2,3.6,4.45,5.95,10.8,7.4,6,6,4.38,4.9$ DM , D , M , 89.09,2009, 17.8, 10.4,3.5,4.15,5.9,10.6,6.8,5.7,6.1,4.11,4.6 $\mathrm{DM}, \mathrm{D}, \mathrm{M}, 90.09,2009,17.9,10.6,3.55,4.45,5.75,10.25,7.2,5.7,6.1,4.15,4.75$ $\mathrm{DM}, \mathrm{D}, \mathrm{M}, 97.09,2009,18.2,10.5,3.5,4.45,5.8,10.5,7.15,6,6.3,4.16,4.75$ $\mathrm{DM}, \mathrm{D}, \mathrm{M}, 98.09,2009,18,10.5,3.6,4.3,5.85,10.25,7.2,5.7,6,4.07,4.75$ $\mathrm{DM}, \mathrm{D}, \mathrm{M}, 99.09,2009,18.8,10.9,3.6,4.55,6.05,10.8,7.2,6.2,6.4,4.45,4.95$ $\mathrm{DM}, \mathrm{D}, \mathrm{M}, 101.09,2009,20.3,10.8,3.6,4.35,5.9,10.7,7.45,6,6.1,4.15,4.75$ $\mathrm{DM}, \mathrm{D}, \mathrm{M}, 102.09,2009,17.7,10.3,3.4,4.15,5.7,10.3,7,5.7,6,4.2,4.55$ $\mathrm{DM}, \mathrm{D}, \mathrm{M}, 104.09,2009,19,10.7,3.55,4.45,5.85,10.6,7.3,5.8,6.1,4.25,4.7$ $\mathrm{DM}, \mathrm{D}, \mathrm{M}, 106.09,2009,18.6,10.7,3.45,4.15,5.7,10.05,6.9,5.8,6.3,4.16,4.65$ $\mathrm{DM}, \mathrm{D}, \mathrm{M}, 253.09,2009,18.1,10.9,3.5,4.3,5.8,10.3,7,5.9,6.4,4.4,4.7$ $\mathrm{DM}, \mathrm{D}, \mathrm{M}, 254.09,2009,21.2,10.9,3.75,4.5,6.55,11.5,7.9,6.3,6.6,4.55,5.2$ $\mathrm{DM}, \mathrm{D}, \mathrm{M}, 1.1,2010,19.4,11.2,3.6,4.6,6.05,10.65,7.3,6.2,6.6,4.35,5.05$ $\mathrm{DM}, \mathrm{D}, \mathrm{M}, 2.1,2010,18.1,10.1,3.4,4.25,5.55,10.25,6.9,5.6,5.7,4.2,4.6$ $\mathrm{DM}, \mathrm{D}, \mathrm{M}, 6.1,2010,20.4,10.5,3.6,4.4,6.1,10.95,7.35,6.2,6.2,4.3,5$ DM , D , M , 10.1,2010,19,11.2,3.6,4.45,5.95,10.75, 7.45,5.9,6,4.4,4.85 $\mathrm{DM}, \mathrm{D}, \mathrm{M}, 11.1,2010,19.4,11.5,3.6,4.5,6,10.3,7.35,6,6.3,4.55,4.8$ $\mathrm{DM}, \mathrm{D}, \mathrm{M}, 12.1,2010,19,11.2,3.5,4.5,5.95,10.65,7.15,5.9,6.2,4.35,4.7$ $\mathrm{DM}, \mathrm{D}, \mathrm{M}, 13.1,2010,18.9,11.1,3.5,4.45,6,10.7,7.25,6.1,6.5,4.3,4.95$ $\mathrm{DM}, \mathrm{D}, \mathrm{M}, 14.1,2010,18.9,11,3.5,4.6,6.15,10.55,7.4,5.9,6.2,4.55,4.95$ $\mathrm{DM}, \mathrm{D}, \mathrm{M}, 31.1,2010,19.1,10.5,3.4,4.45,5.7,10.45,7.25,5.8,6.2,4.15,4.65$ $\mathrm{DM}, \mathrm{D}, \mathrm{M}, 51.1,2010,18.7,10.6,3.6,4.45,5.85,10.5,7.35,6,6.3,4.45,4.85$ $\mathrm{DM}, \mathrm{D}, \mathrm{M}, 52.1,2010,19.4,10.9,3.6,4.45,5.9,10.8,7.45,6.1,6.2,4.3,5$ $\mathrm{DM}, \mathrm{D}, \mathrm{M}, 56.1,2010,18.9,11.1,3.5,4.5,6.25,10.25,7.1,6,6.3,4.35,5$ $\mathrm{DM}, \mathrm{D}, \mathrm{M}, 73.1,2010,18.6,11.3,3.65,4.55,6.25,10.65,7.3,6.1,6.3,4.3,5$ $\mathrm{DM}, \mathrm{D}, \mathrm{M}, 74.1,2010,18,10.3,3.5,4.25,5.9,10.5,7.05,5.7,6.2,4.2,4.65$ $\mathrm{DM}, \mathrm{D}, \mathrm{M}, 75.1,2010,18,10.4,3.45,4.25,5.6,10.15,7.1,5.7,5.9,4.2,4.6$ $\mathrm{DM}, \mathrm{D}, \mathrm{M}, 76.1,2010,18.3,10.3,3.45,4.15,5.65,10.35,6.95,5.9,6.1,4.15,4.75$ $\mathrm{DM}, \mathrm{D}, \mathrm{M}, 77.1,2010,19.5,10.6,3.5,4.4,6,10.75,7.4,5.9,6.2,4.3,4.8$ $\mathrm{DM}, \mathrm{D}, \mathrm{M}, 78.1,2010,18.6,10.2,3.3,3.95,5.55,10,7,5.7,5.7,4,4.35$ $\mathrm{DM}, \mathrm{D}, \mathrm{M}, 89.1,2010,19.2,11.4,3.65,4.6,6.25,11.3,7.35,6,6.2,4.35,5$ $\mathrm{DM}, \mathrm{D}, \mathrm{M}, 91.1,2010,18.9,11.4,3.55,4.4,5.9,10.8,7.1,6.1,6.3,4.25,4.8$ $\mathrm{DM}, \mathrm{D}, \mathrm{M}, 92.1,2010,17.6,10.4,3.4,4.1,5.55,10,7,5.7,5.8,4.1,4.6$ $\mathrm{DM}, \mathrm{D}, \mathrm{M}, 93.1,2010,18.4,11,3.55,4.3,5.75,10.6,7.2,5.8,6.2,4.15,4.8$

$\mathrm{DM}, \mathrm{D}, \mathrm{M}, 94.1,2010,18.6,11,3.5,4.4,6.05,10.65,7.2,6,6.2,4.25,4.9$ 
DM , D , M , 95.1,2010,18.5, 11.1,3.5,4.3, 5.8, 10.55, 7.2,6,6.2,4.15,4.75

$\mathrm{DM}, \mathrm{D}, \mathrm{M}, 96.1,2010,18.3,11,3.55,4.4,5.9,10.55,7.25,5.9,6.2,4.3,4.85$ $\mathrm{DM}, \mathrm{D}, \mathrm{M}, 97.1,2010,18.6,10.7,3.6,4.4,6,10.55,7.2,5.9,6.1,4.3,4.7$

$\mathrm{DM}, \mathrm{D}, \mathrm{M}, 98.1,2010,19.3,10.5,3.5,4.15,5.8,10.45,7,5.8,6.1,4.2,4.75$

$\mathrm{DM}, \mathrm{D}, \mathrm{M}, 99.1,2010,18.5,11,3.6,4.35,5.9,10.3,7.2,5.8,6.1,4.35,4.9$

$\mathrm{DM}, \mathrm{D}, \mathrm{M}, 119.1,2010,18.7,10.4,3.45,4.2,5.6,10.1,6.7,5.6,5.9,4.5,4.5$

$\mathrm{DM}, \mathrm{D}, \mathrm{M}, 121.1,2010,19,10.6,3.5,4.35,5.75,10.5,7.05,5.9,6.1,4.75,4.75$

$\mathrm{DM}, \mathrm{D}, \mathrm{M}, 122.1,2010,20,10.9,3.6,4.35,5.8,10.8,7.1,6,6.2,4.8,4.8$

$\mathrm{DM}, \mathrm{D}, \mathrm{M}, 123.1,2010,18.8,10.9,3.6,4.4,5.9,10.5,7.4,6,6.3,4.8,4.8$

$\mathrm{DM}, \mathrm{D}, \mathrm{M}, 134.1,2010,18.5,10.5,3.45,4.15,5.7,10.2,6.8,5.8,6,4.1,4.65$

DM, D , M , 149.1,2010,18.6,10.8,3.55,4.35,5.75,10.7, 7.15,6,6.2,4.5, 4.9

$\mathrm{DM}, \mathrm{D}, \mathrm{M}, 152.1,2010,18.2,11.3,3.7,4.55,6.1,10.7,7.3,6,6.2,4.3,4.9$

$\mathrm{DM}, \mathrm{D}, \mathrm{M}, 155.1,2010,17.2,10.3,3.45,4.15,5.65,10,6.8,5.6,6,4.1,4.6$ 
Appendix B: Data and computer files for geometric morphometric analysis of mandibles.

B.1) TPS-format file of raw landmark coordinates for left mandible of 108 specimens of Omus.

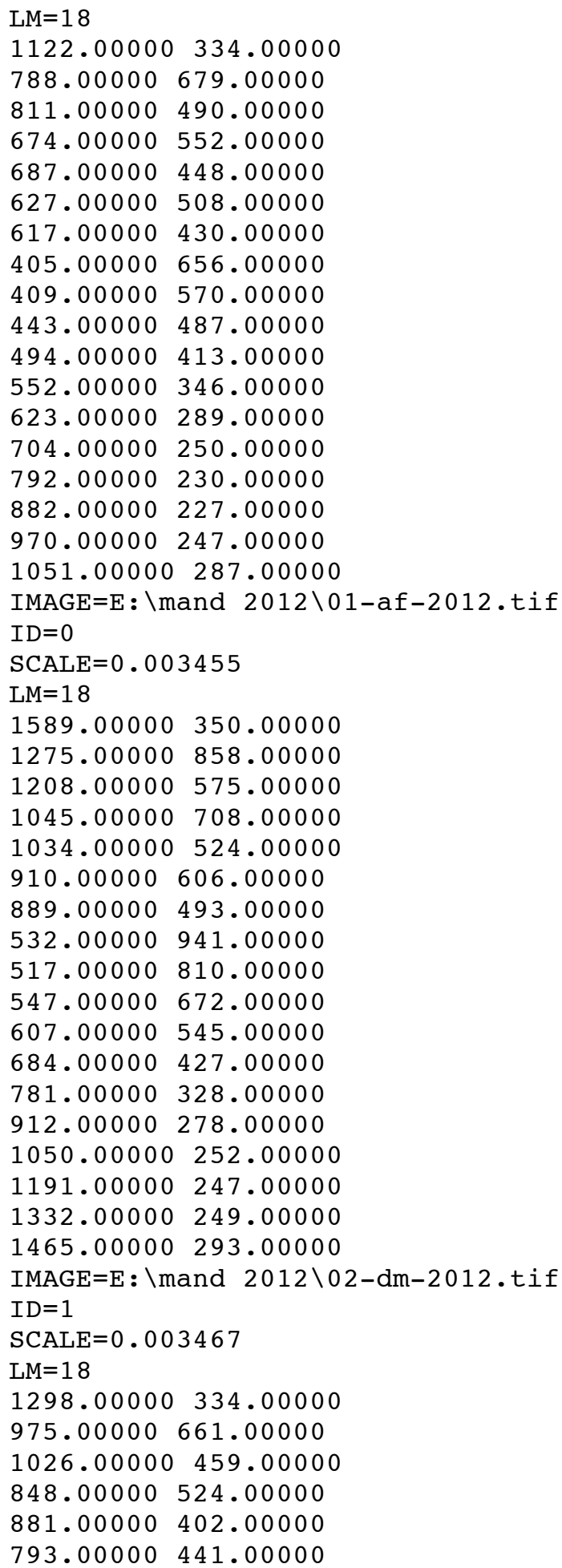




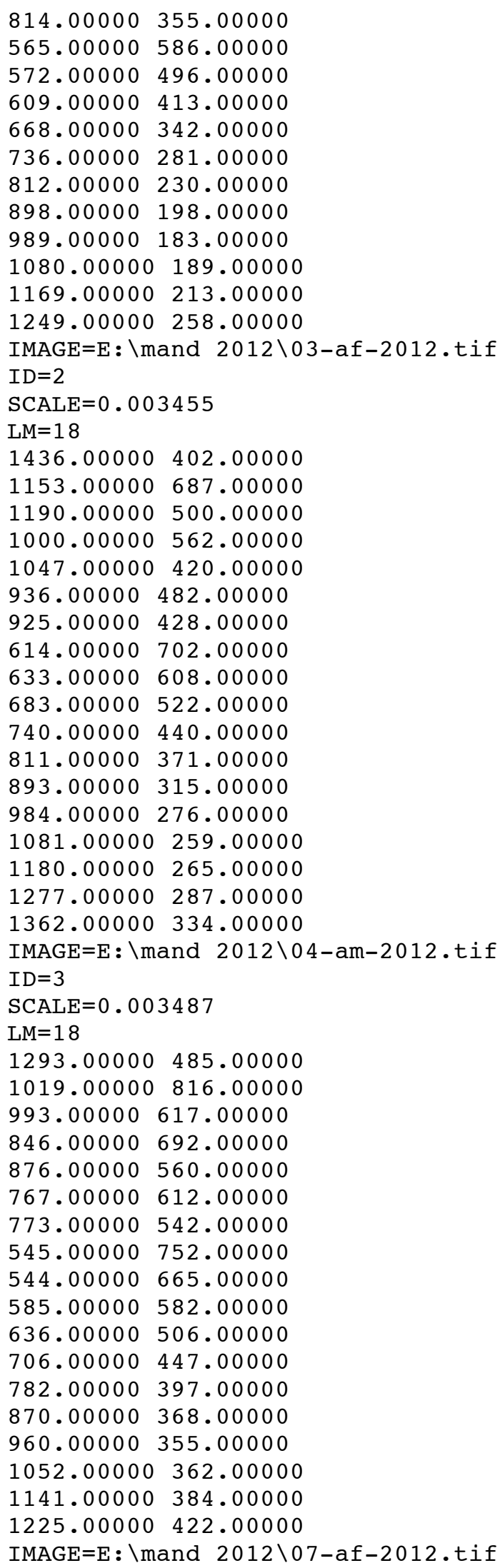




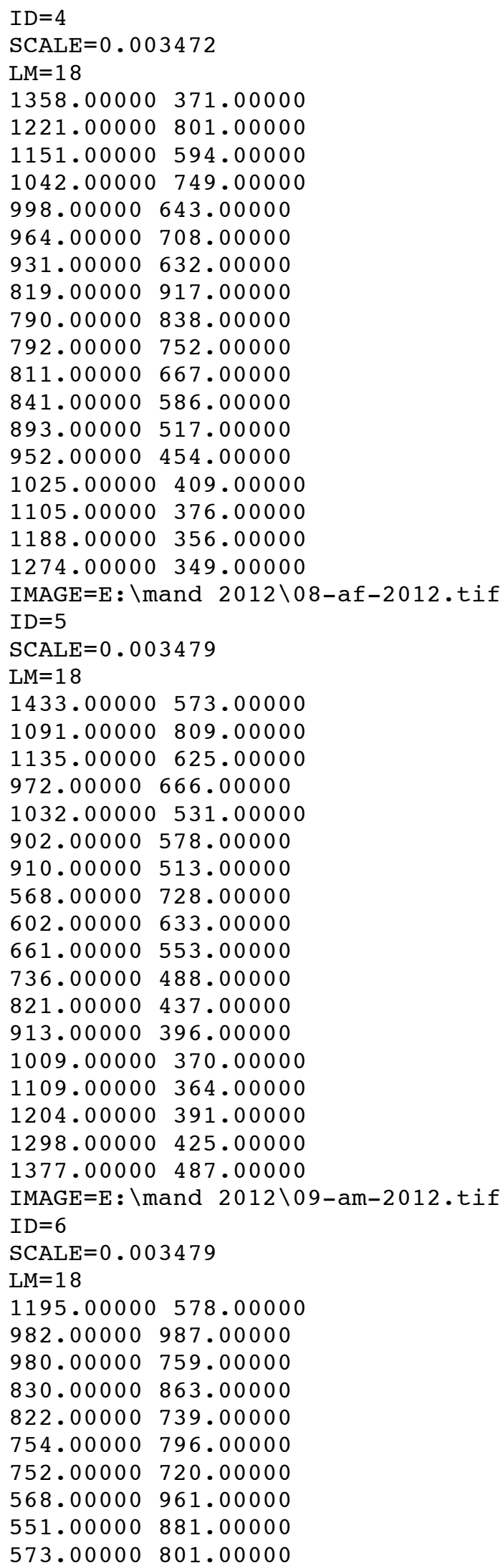




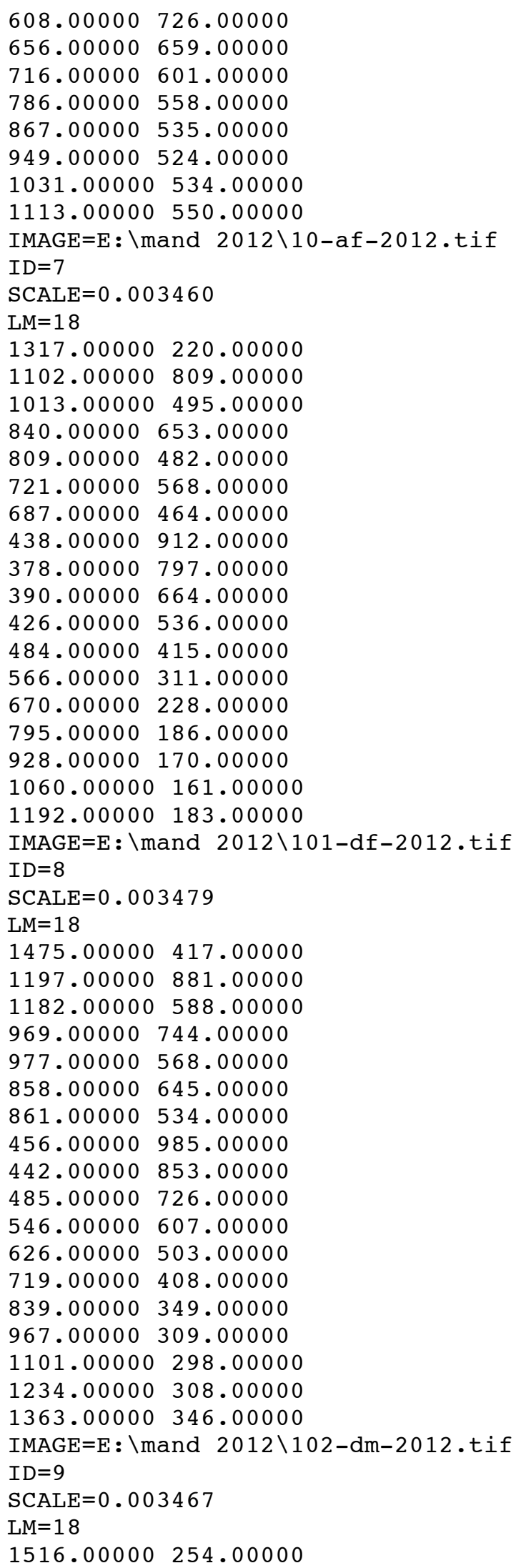




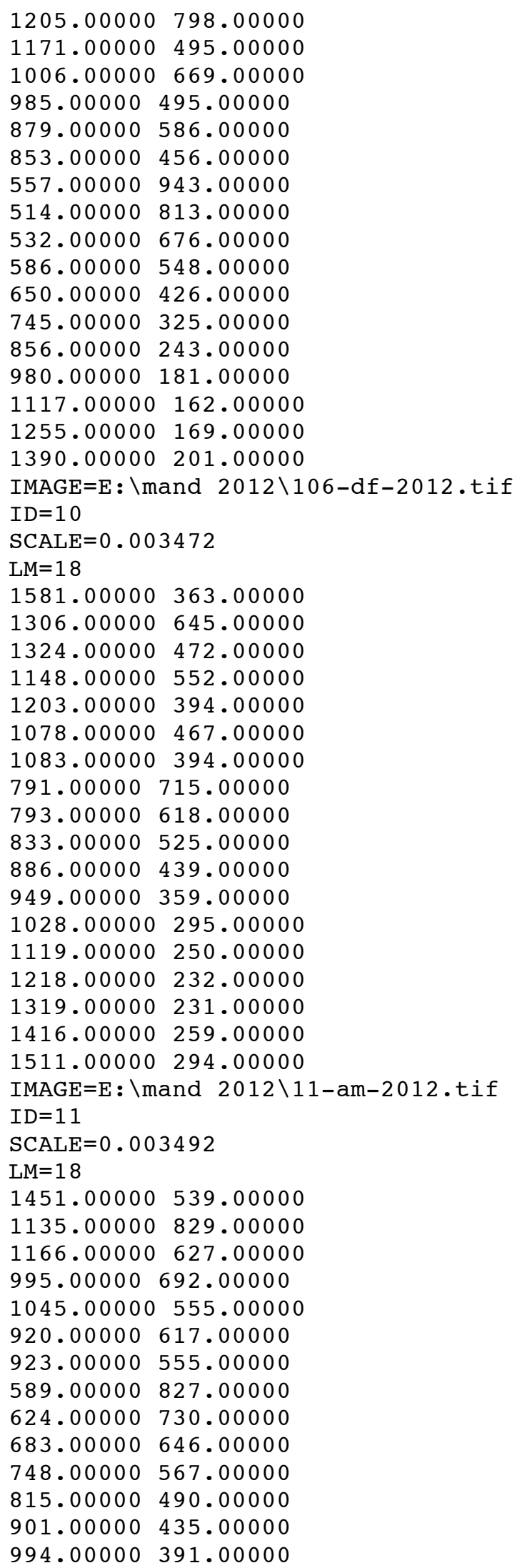




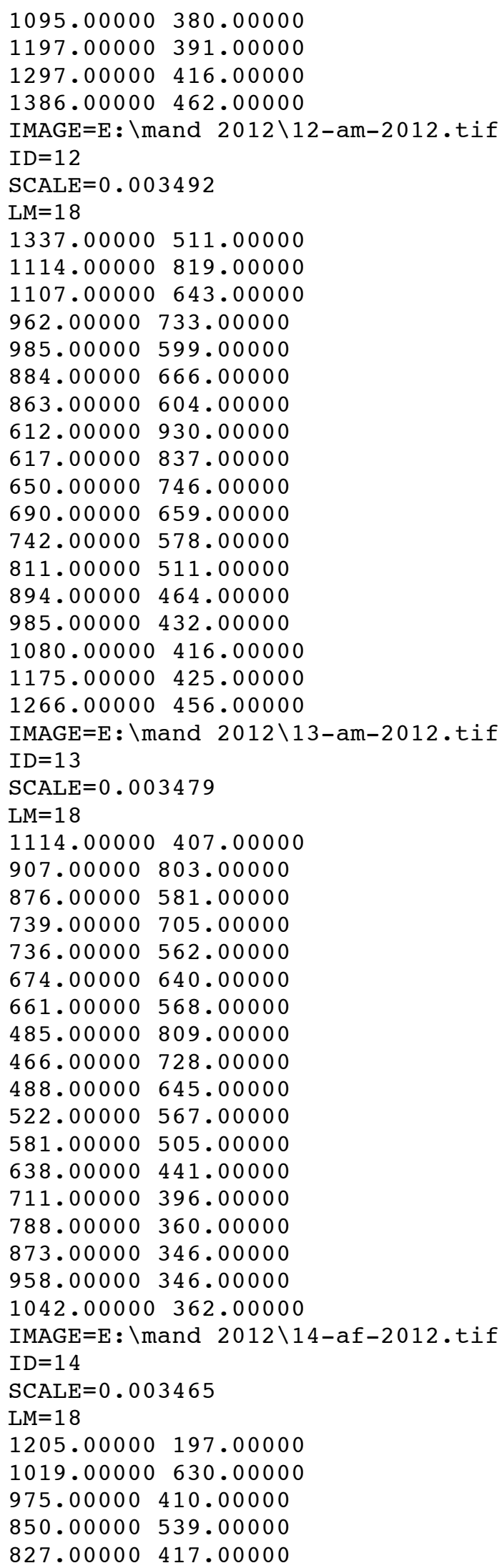




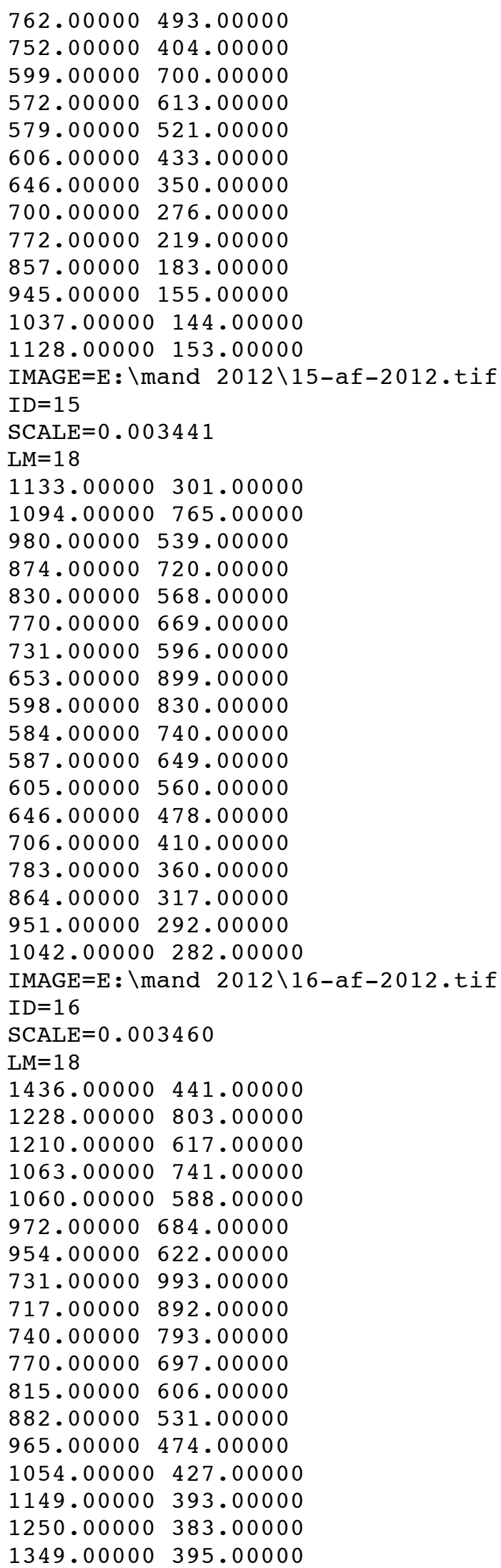




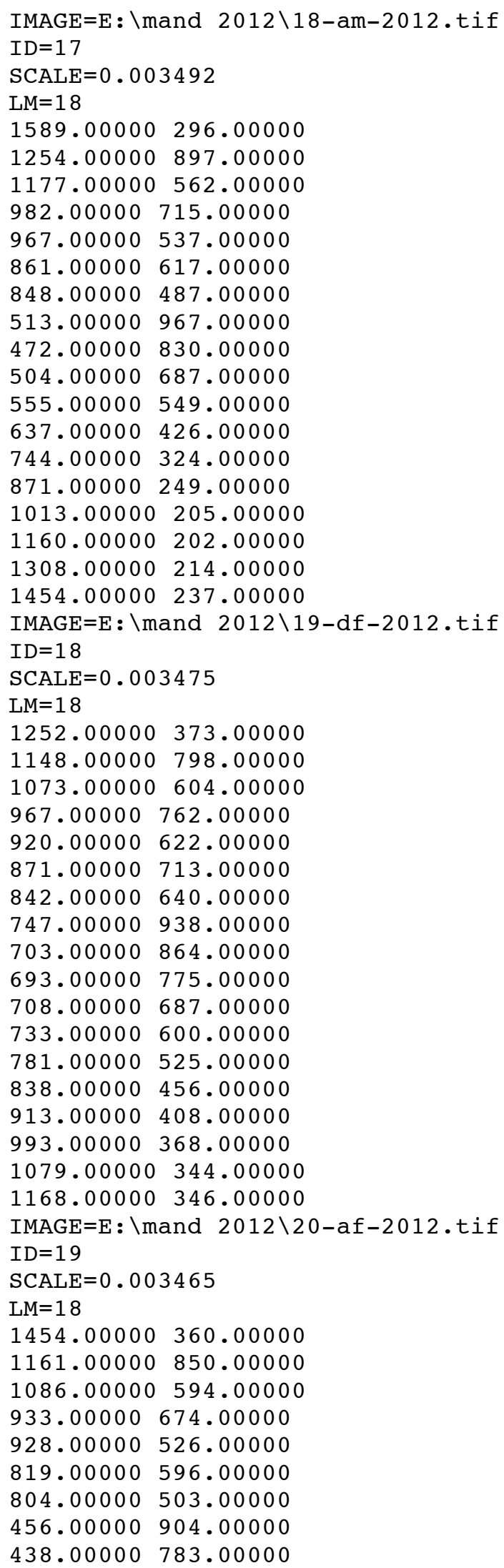




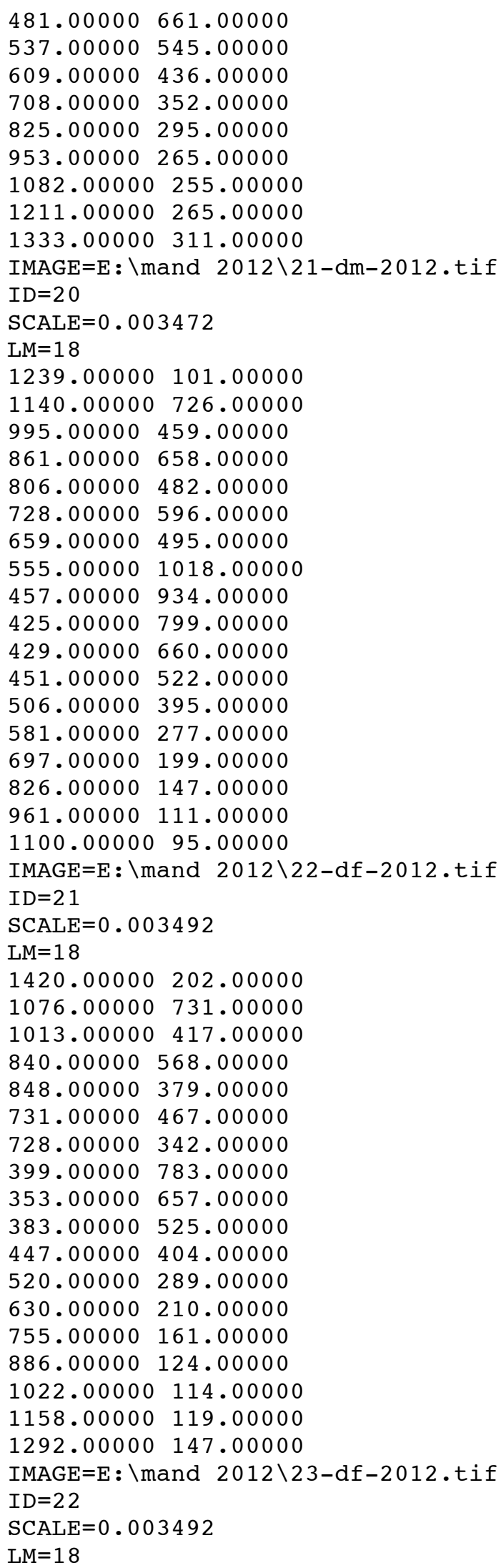




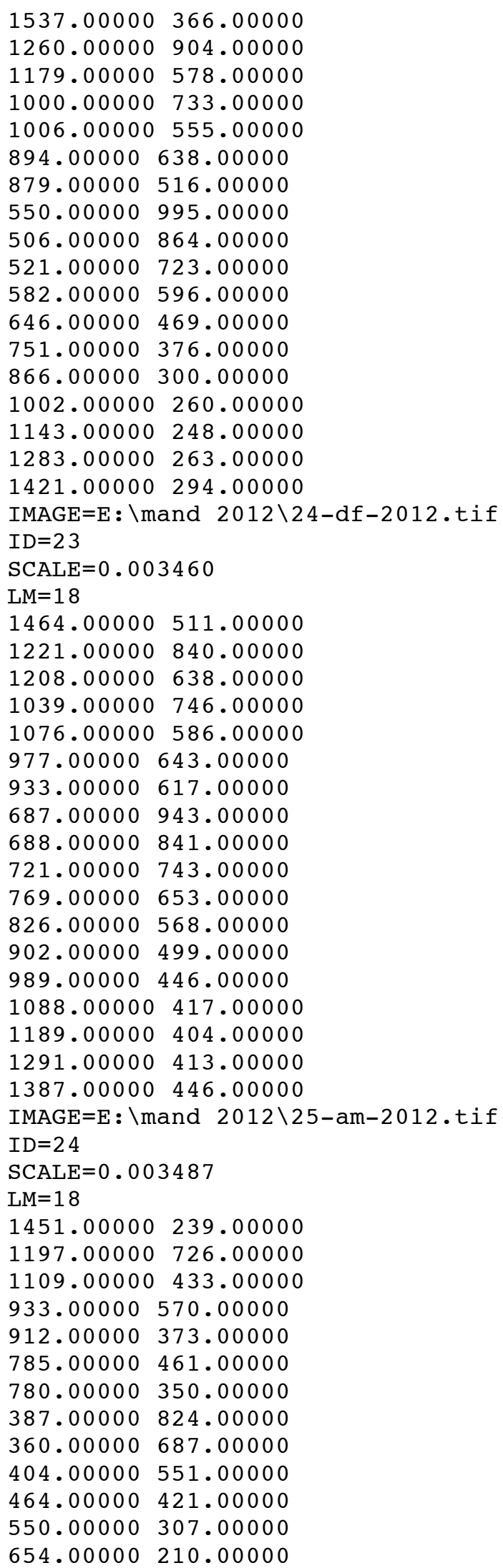




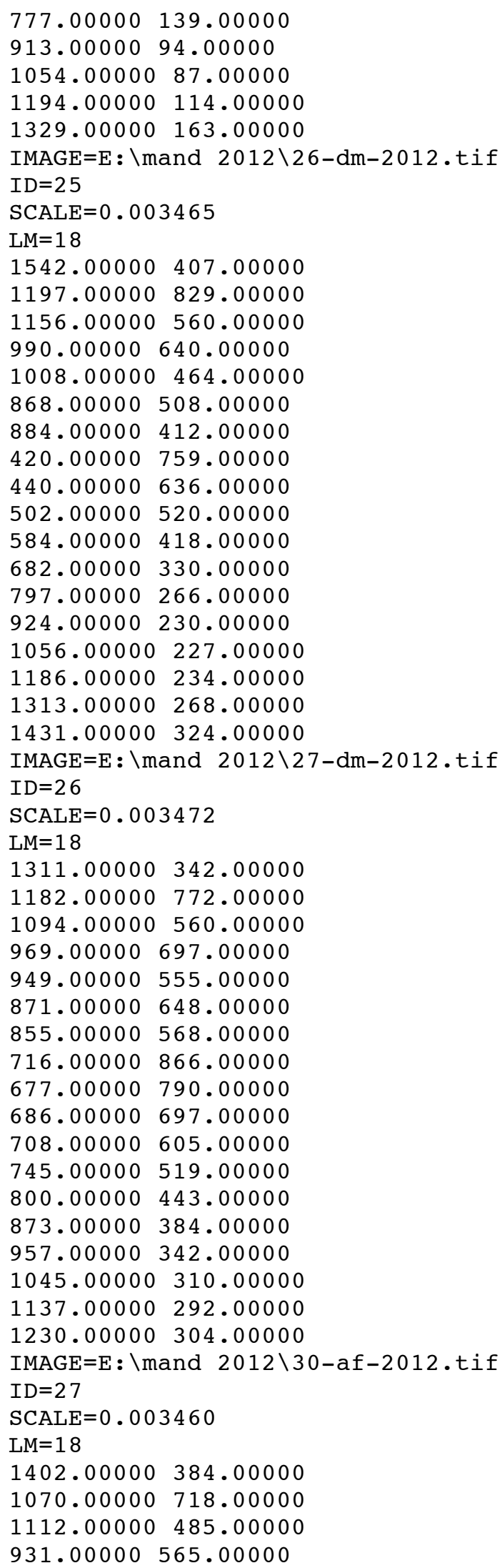




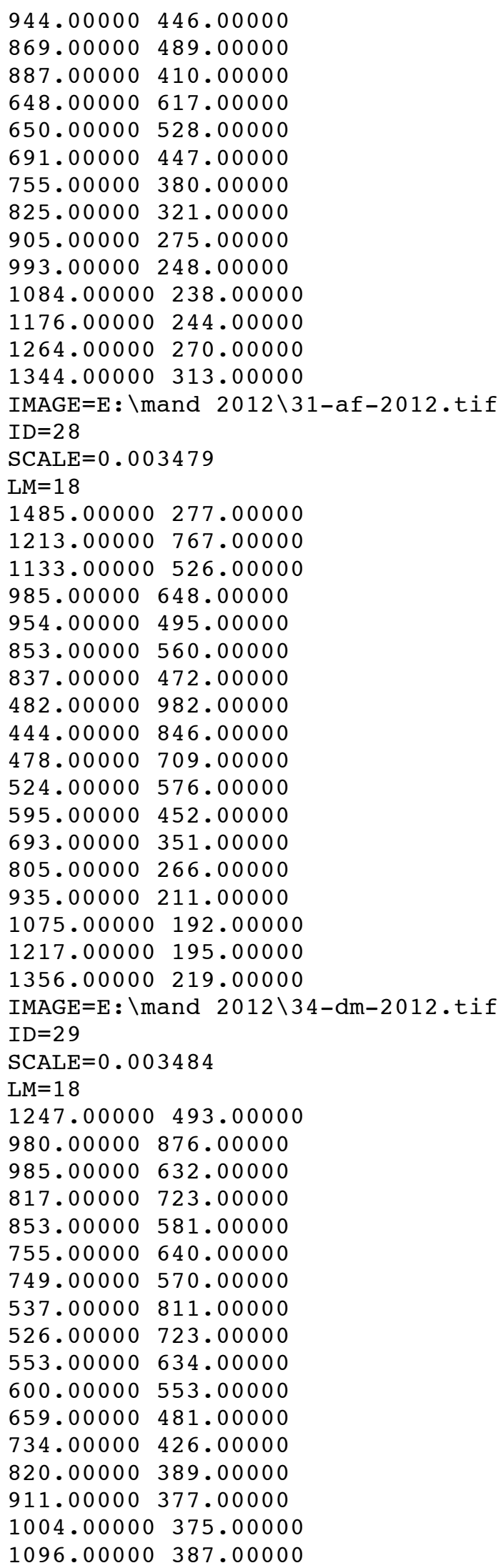




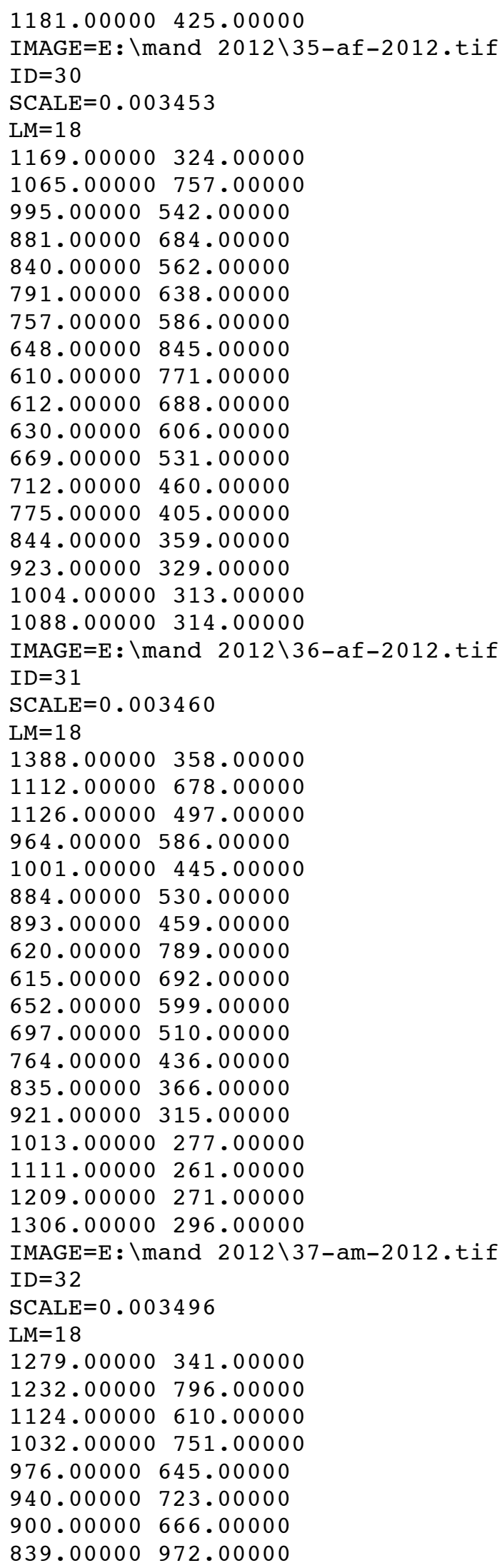




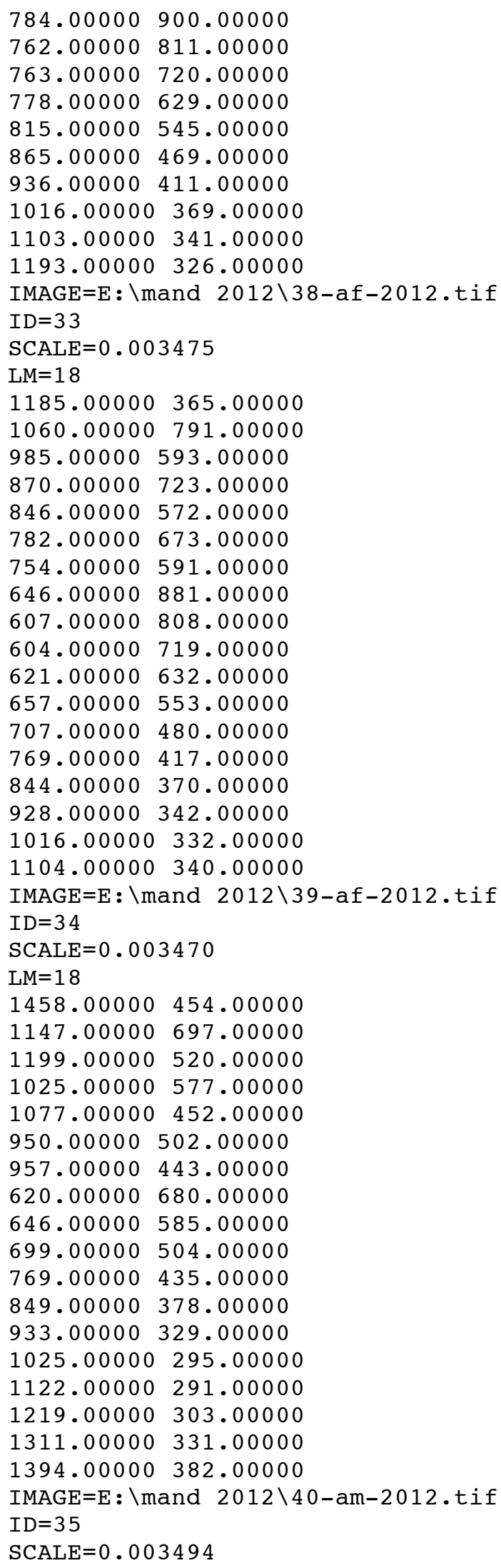




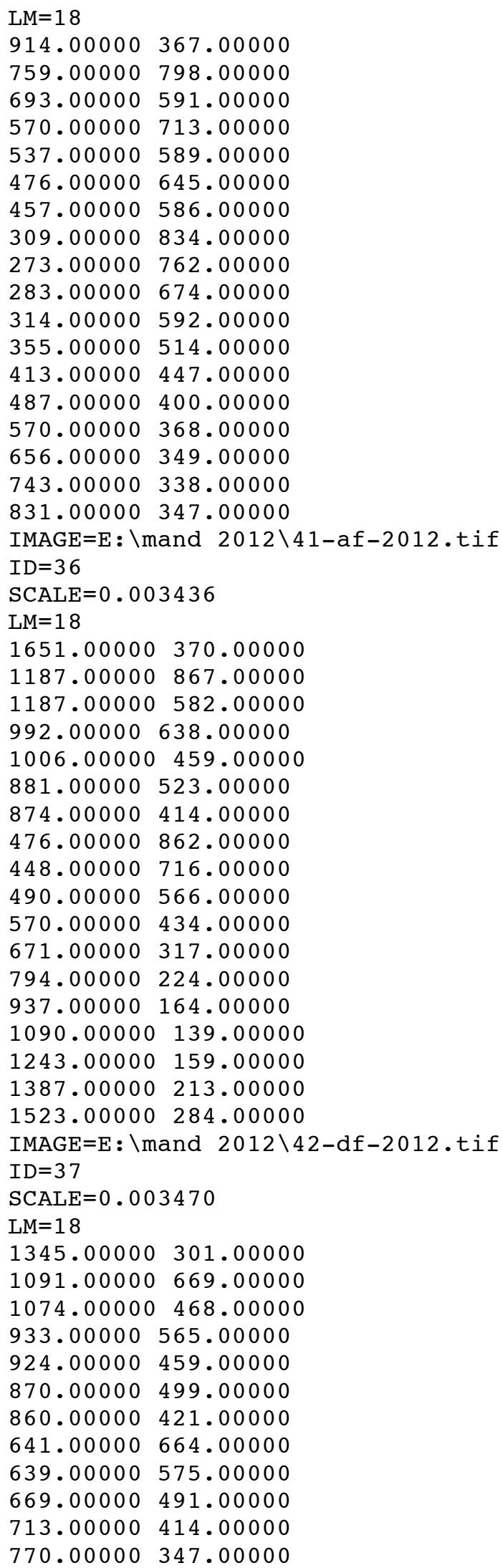




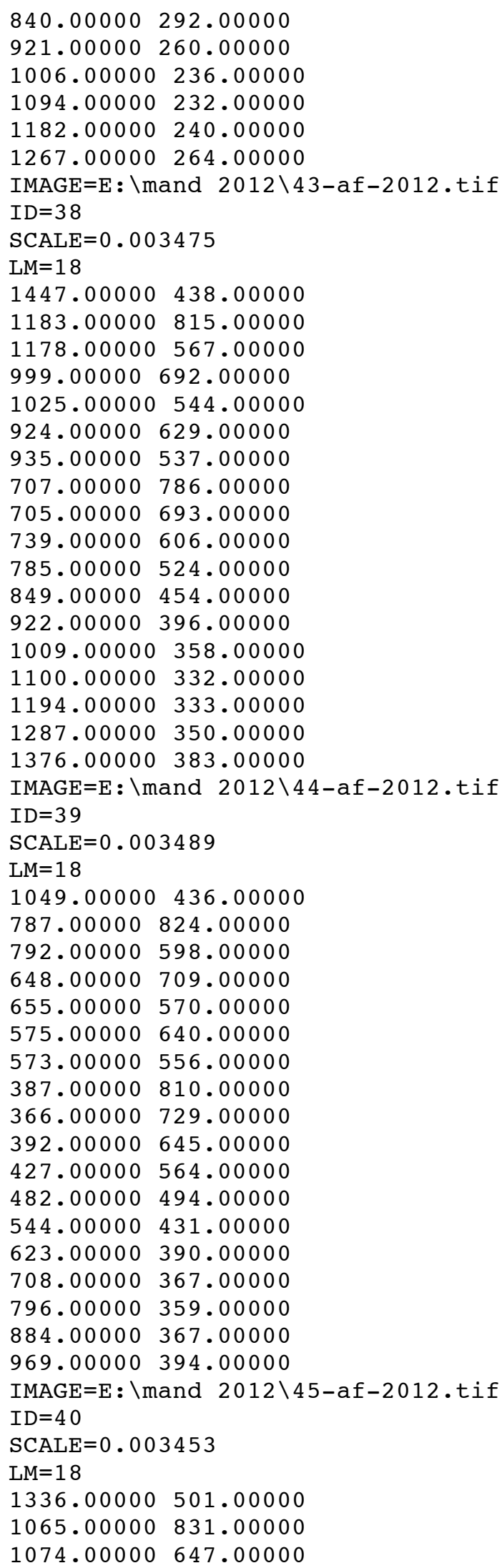




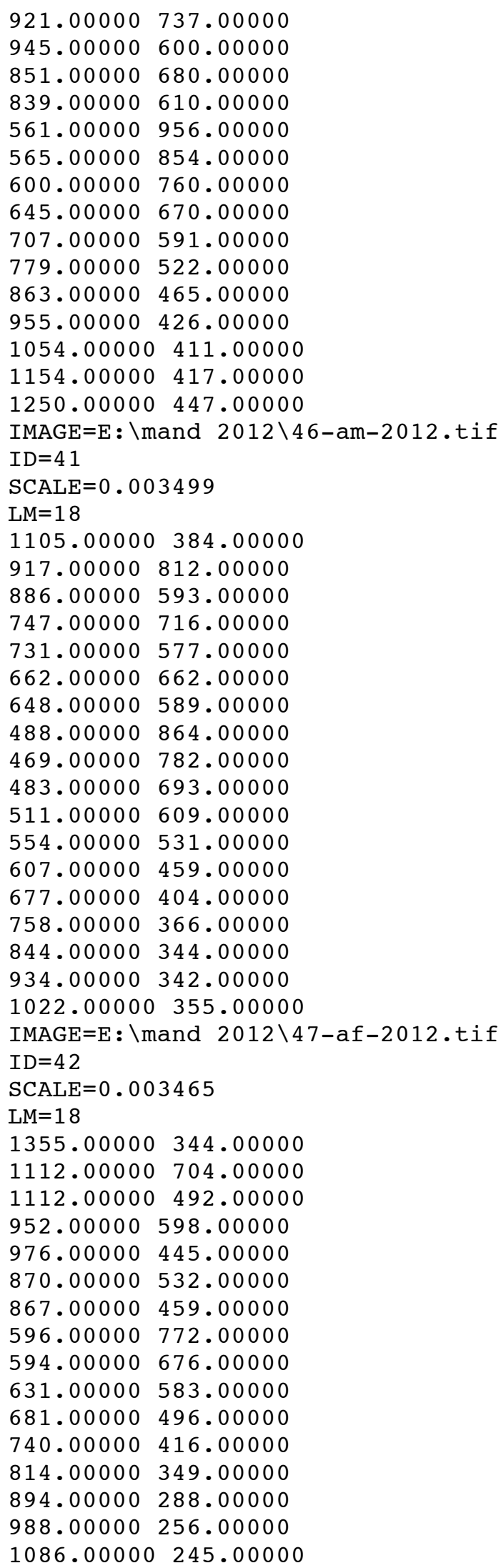


$1186.00000 \quad 254.00000$

$1282.00000 \quad 281.00000$

IMAGE $=\mathrm{E}: \backslash$ mand $2012 \backslash 48-$ am-2012.tif

$I D=43$

SCALE $=0.003479$

$\mathrm{LM}=18$

1331.00000282 .00000

$1103.00000 \quad 666.00000$

$1084.00000 \quad 461.00000$

$935.00000 \quad 572.00000$

$940.00000 \quad 426.00000$

855.00000506 .00000

$860.00000 \quad 424.00000$

655.00000690 .00000

636.00000607 .00000

$655.00000 \quad 518.00000$

$699.00000 \quad 440.00000$

$752.00000 \quad 365.00000$

$814.00000 \quad 298.00000$

893.00000251 .00000

981.00000226 .00000

$1071.00000 \quad 217.00000$

1163.00000221 .00000

$1252.00000 \quad 240.00000$

IMAGE $=E: \backslash \operatorname{mand} 2012 \backslash 49-a f-2012$.tif

$\mathrm{ID}=44$

SCALE $=0.003465$

$\mathrm{LM}=18$

$1169.00000 \quad 436.00000$

1006.00000869 .00000

$973.00000 \quad 638.00000$

827.00000768 .00000

811.00000638 .00000

742.00000711 .00000

738.00000633 .00000

$570.00000 \quad 897.00000$

550.00000813 .00000

$564.00000 \quad 729.00000$

595.00000650 .00000

$636.00000 \quad 575.00000$

$690.00000 \quad 512.00000$

$754.00000 \quad 456.00000$

$833.00000 \quad 424.00000$

$917.00000 \quad 405.00000$

$1002.00000 \quad 402.00000$

$1087.00000 \quad 407.00000$

IMAGE $=E: \backslash$ mand $2012 \backslash 51-a f-2012$.tif

$\mathrm{ID}=45$

SCALE $=0.003475$

$\mathrm{LM}=18$

$1171.00000 \quad 308.00000$

938.00000718 .00000

914.00000511 .00000

$785.00000 \quad 636.00000$

$778.00000 \quad 499.00000$

$719.00000 \quad 570.00000$

$702.00000 \quad 499.00000$ 


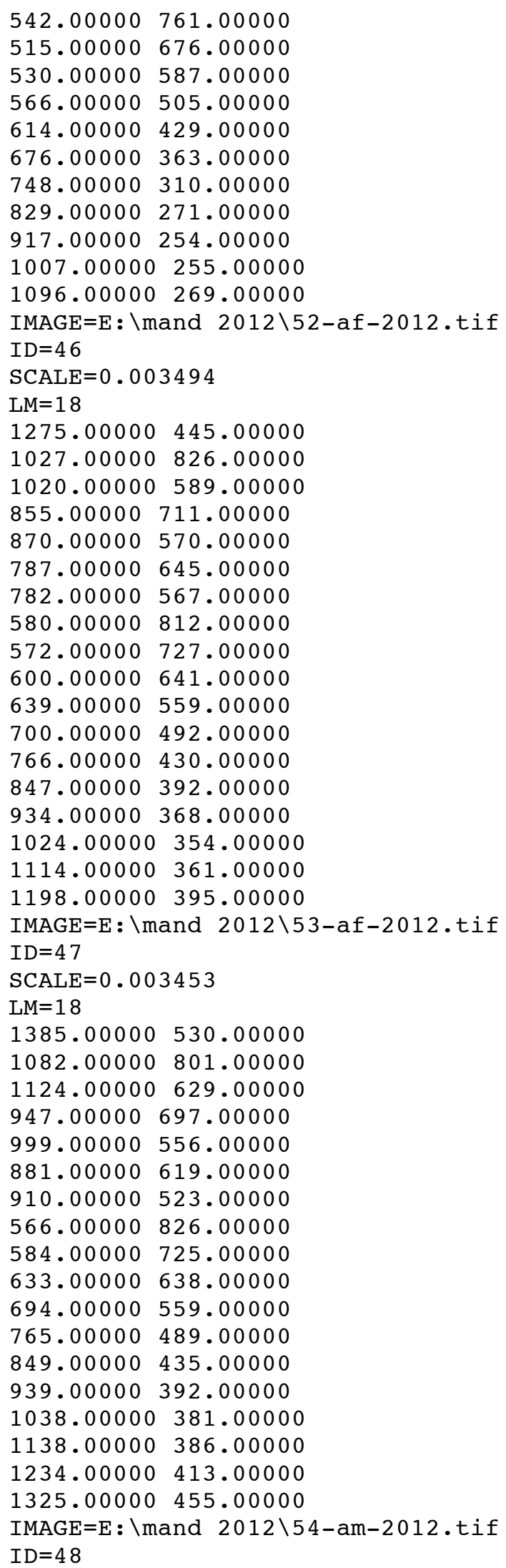




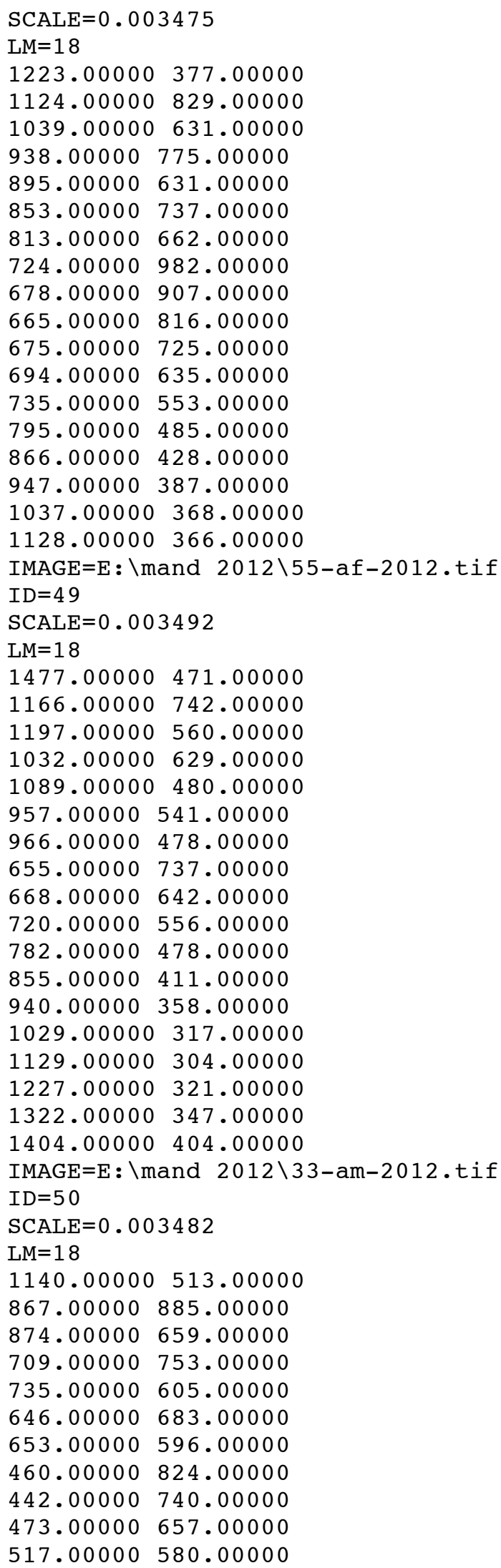




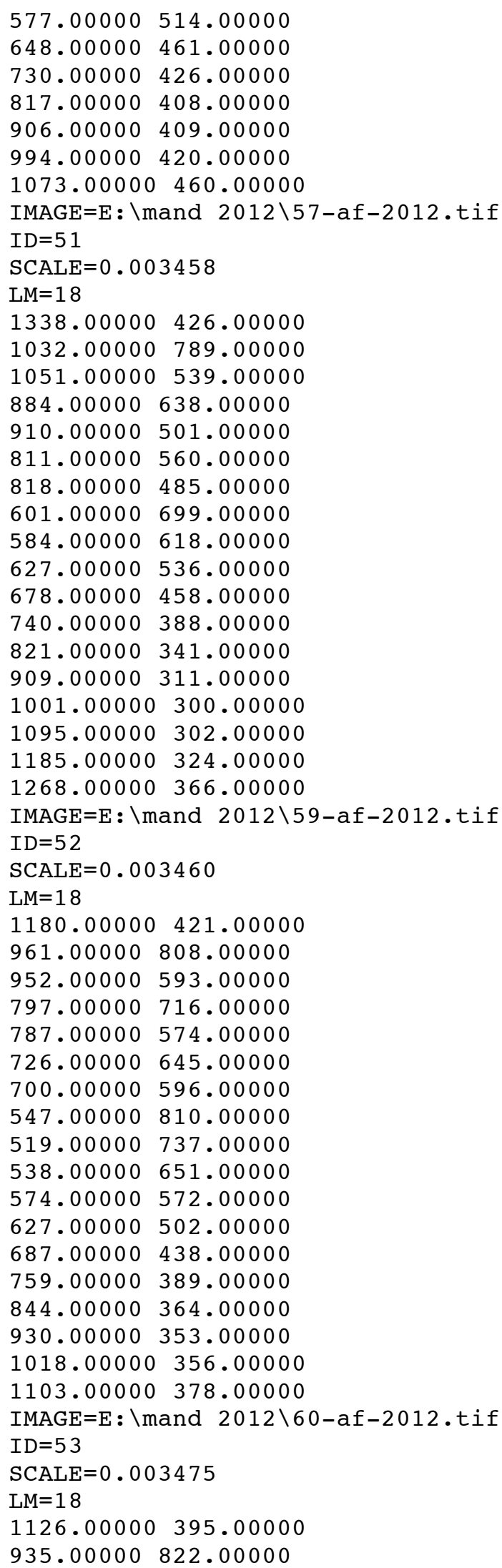




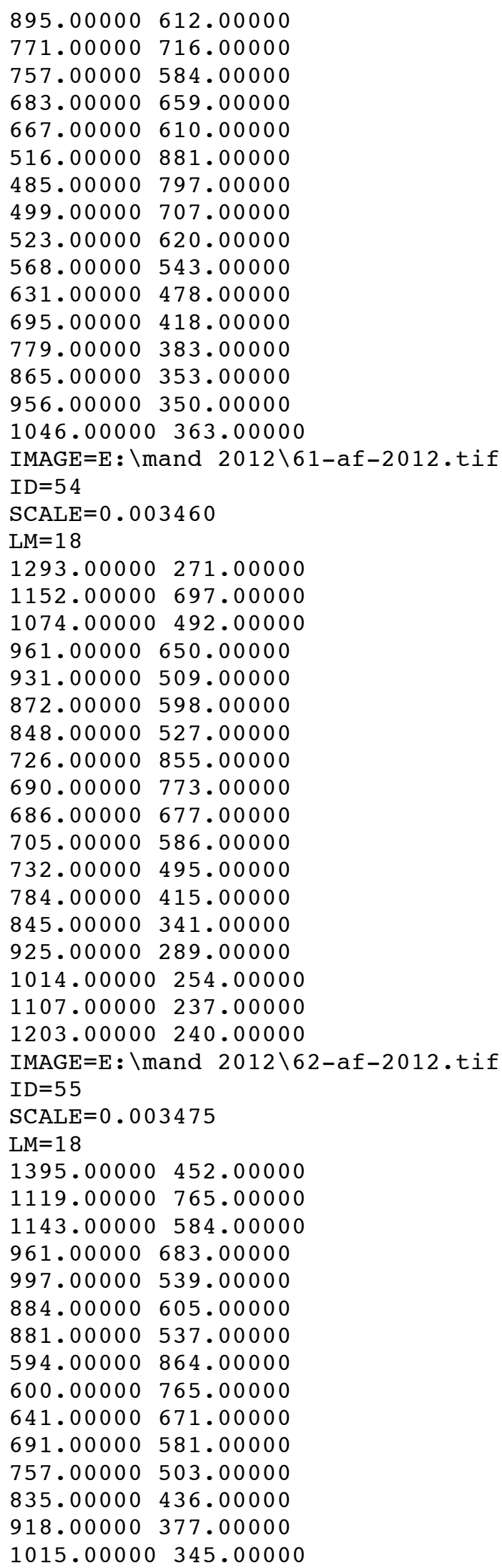




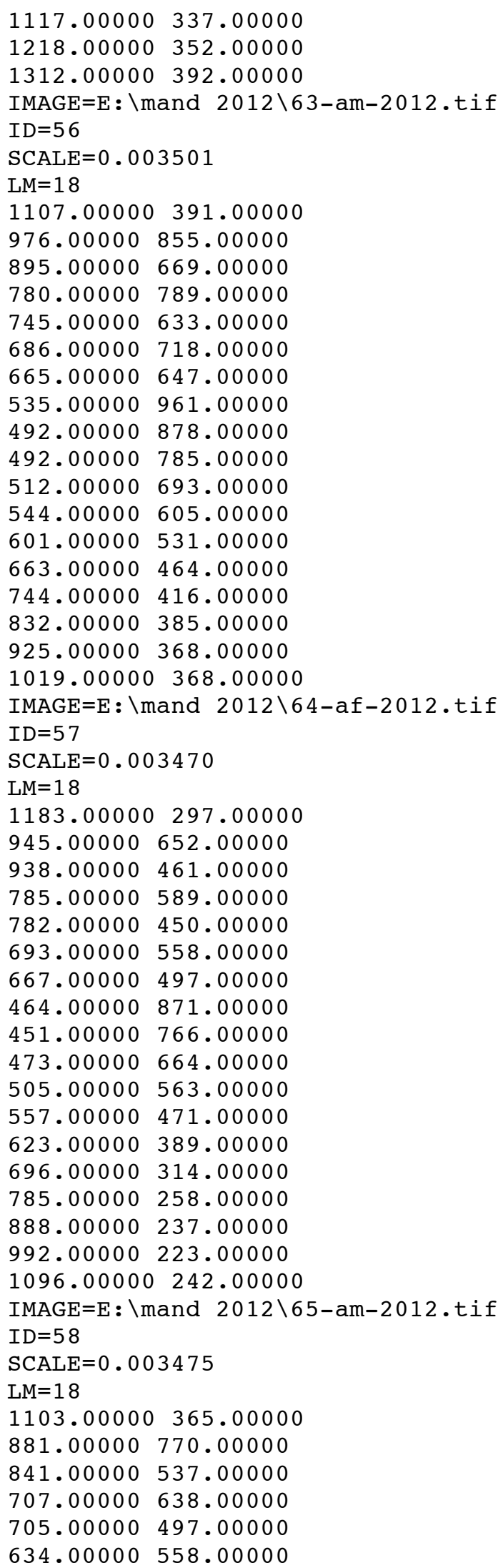




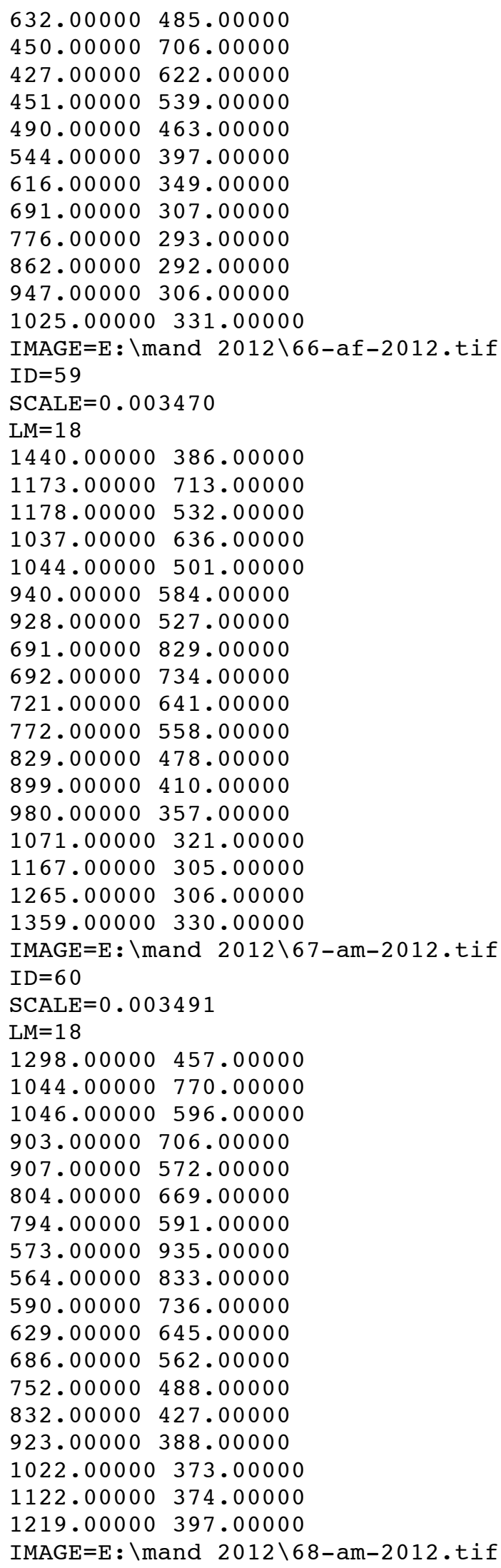




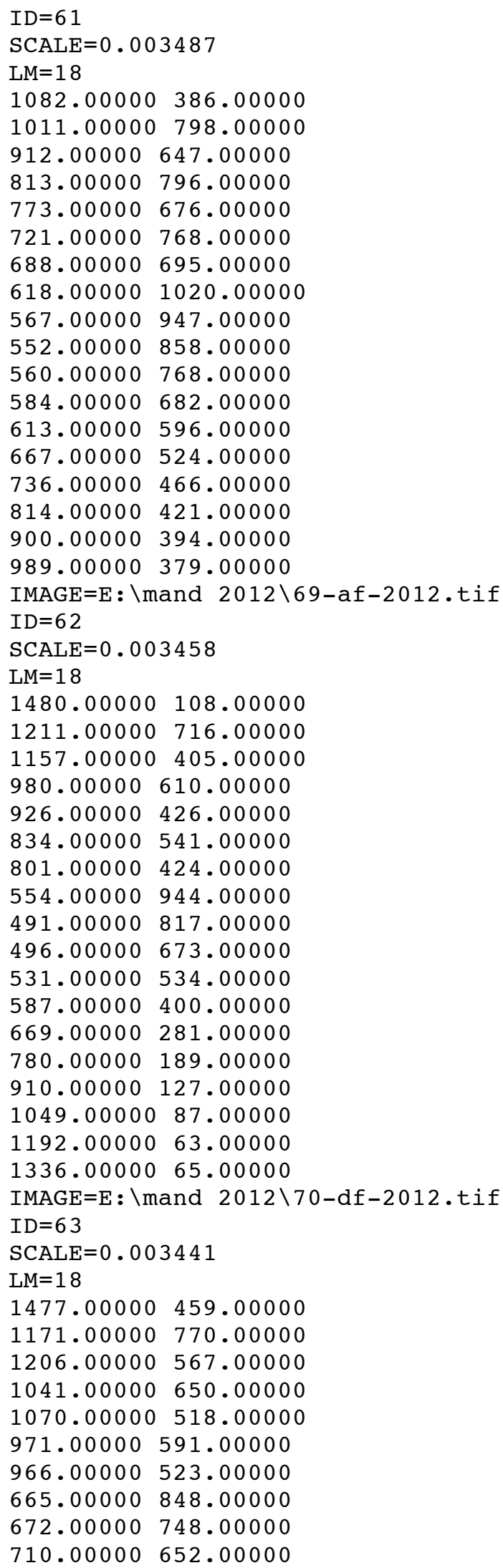




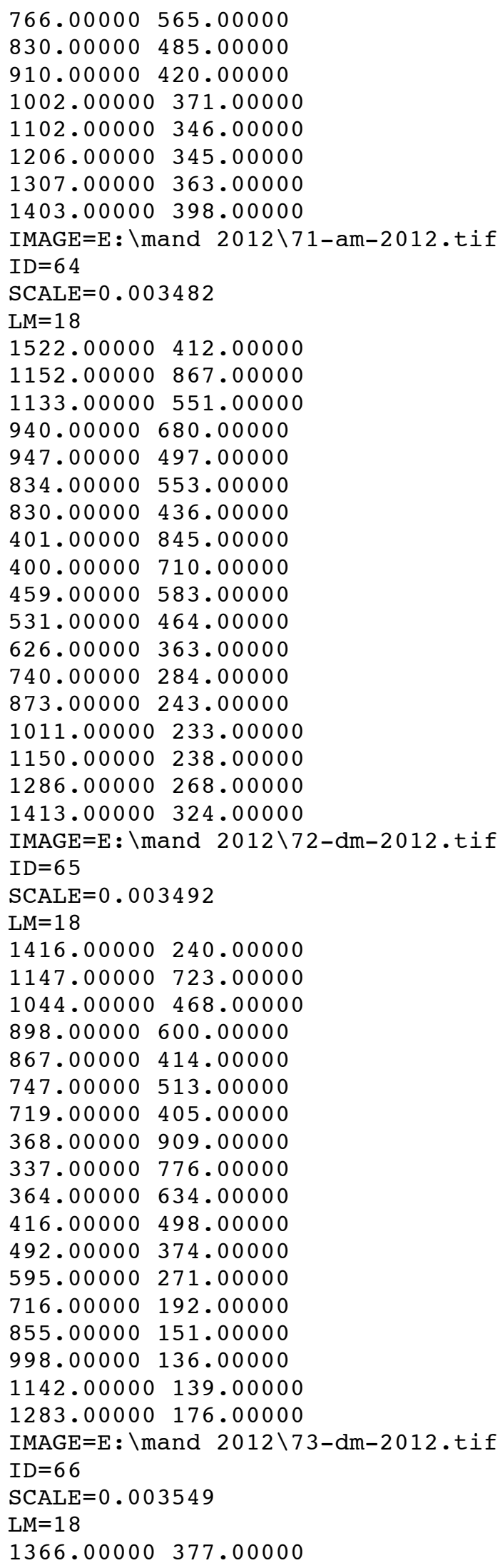




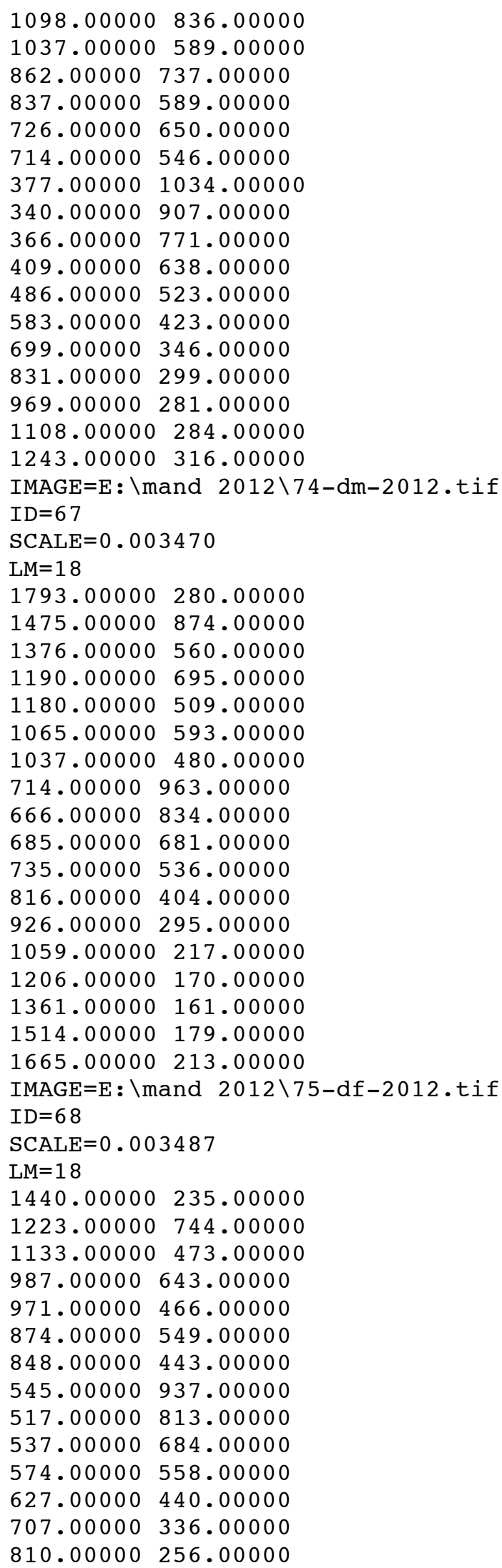




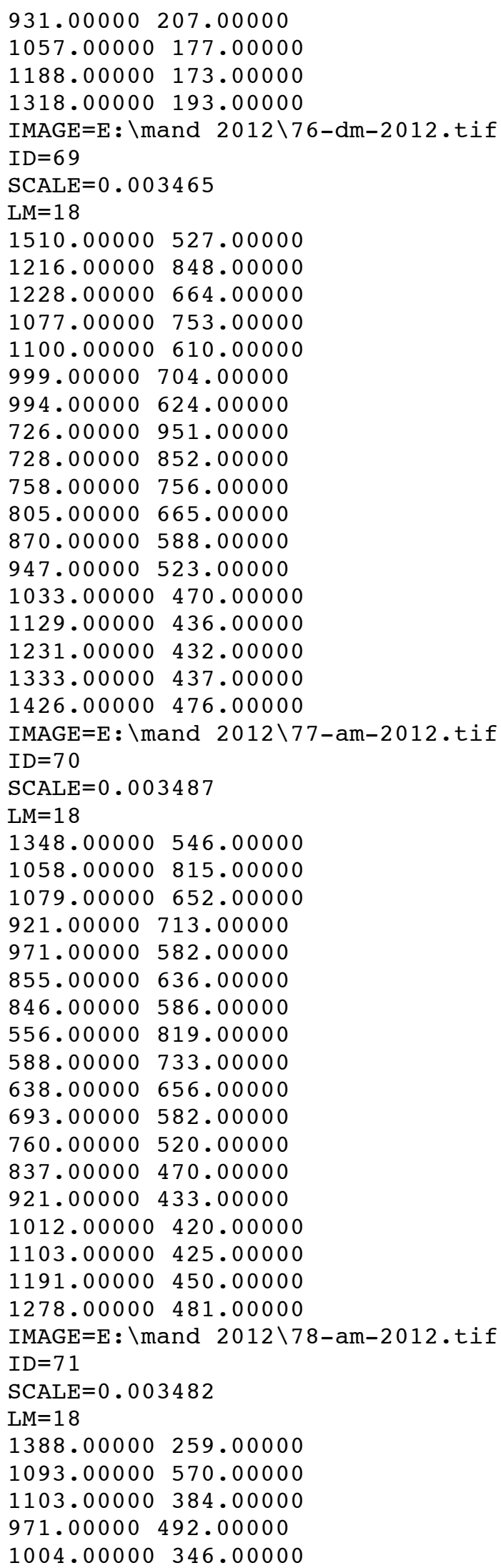




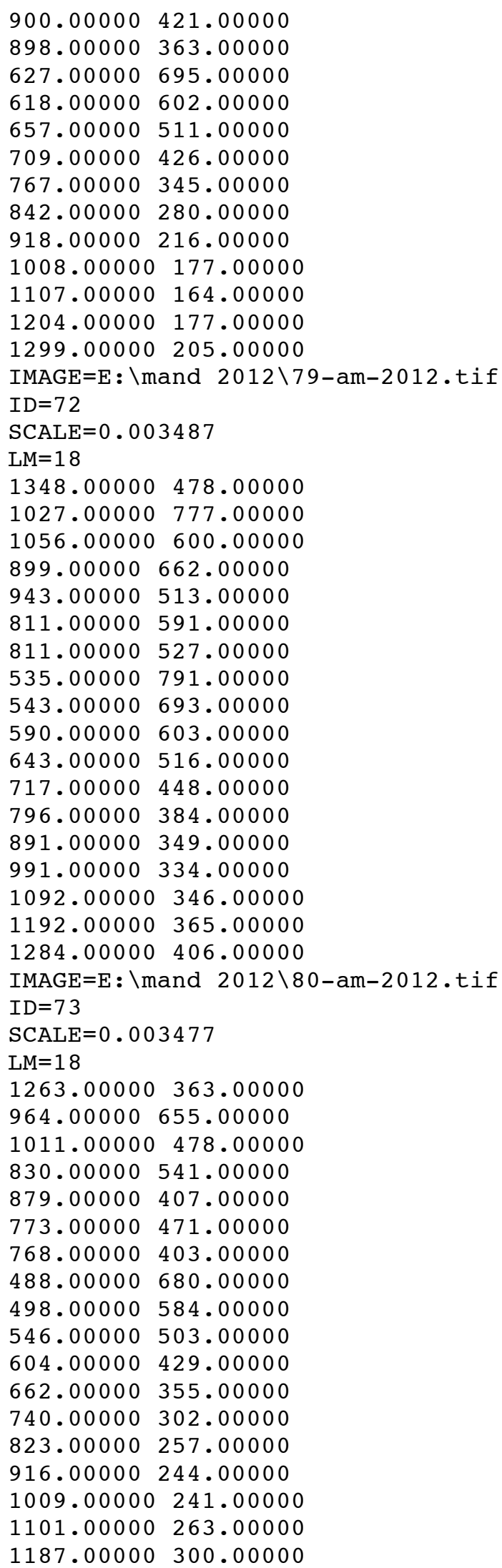




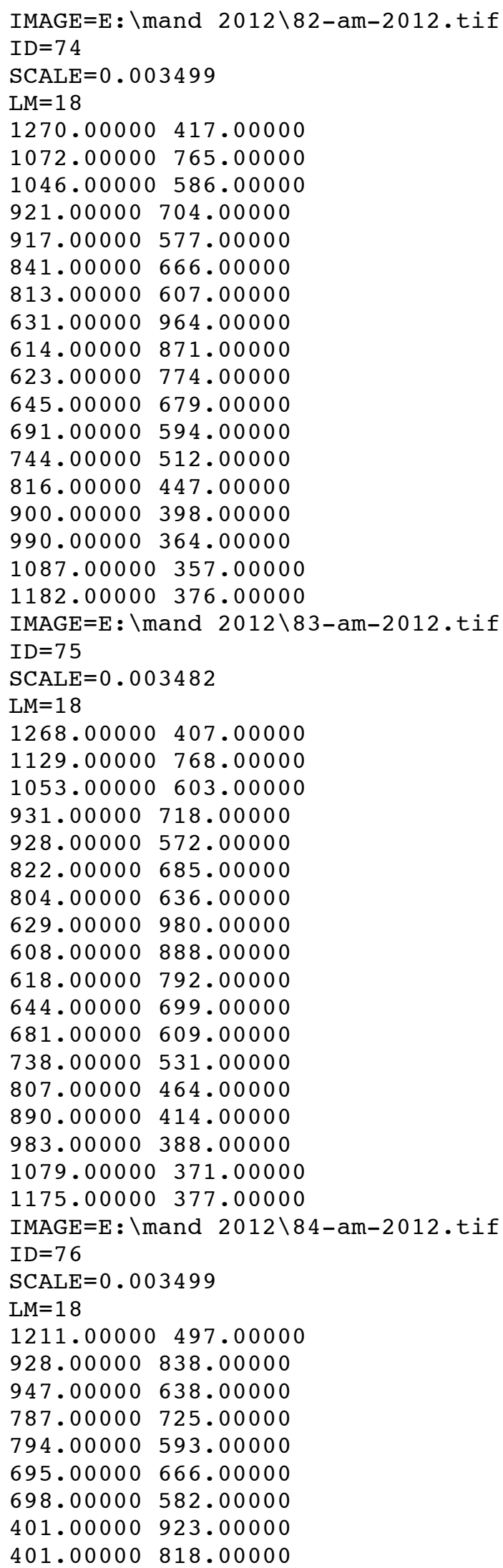




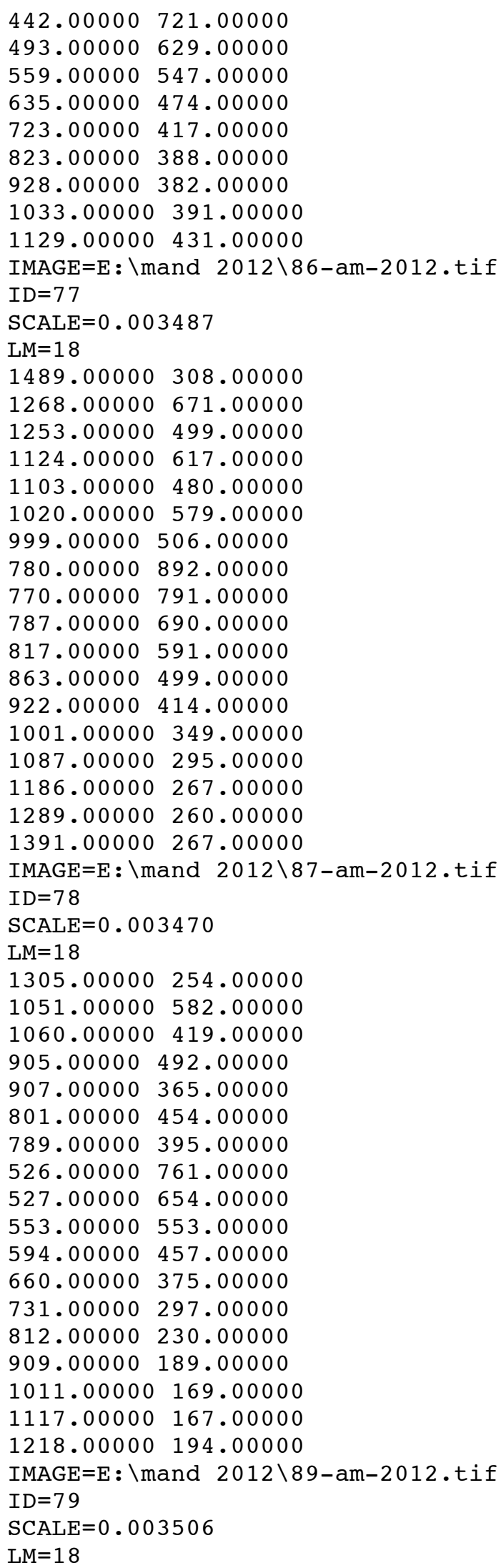




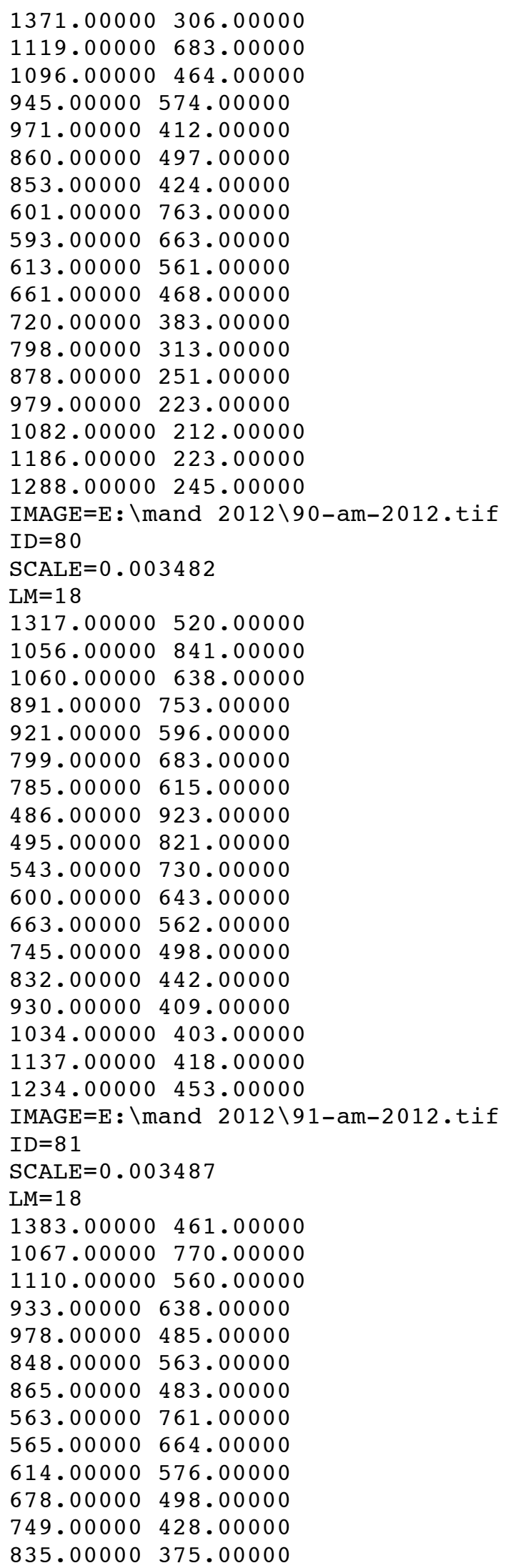




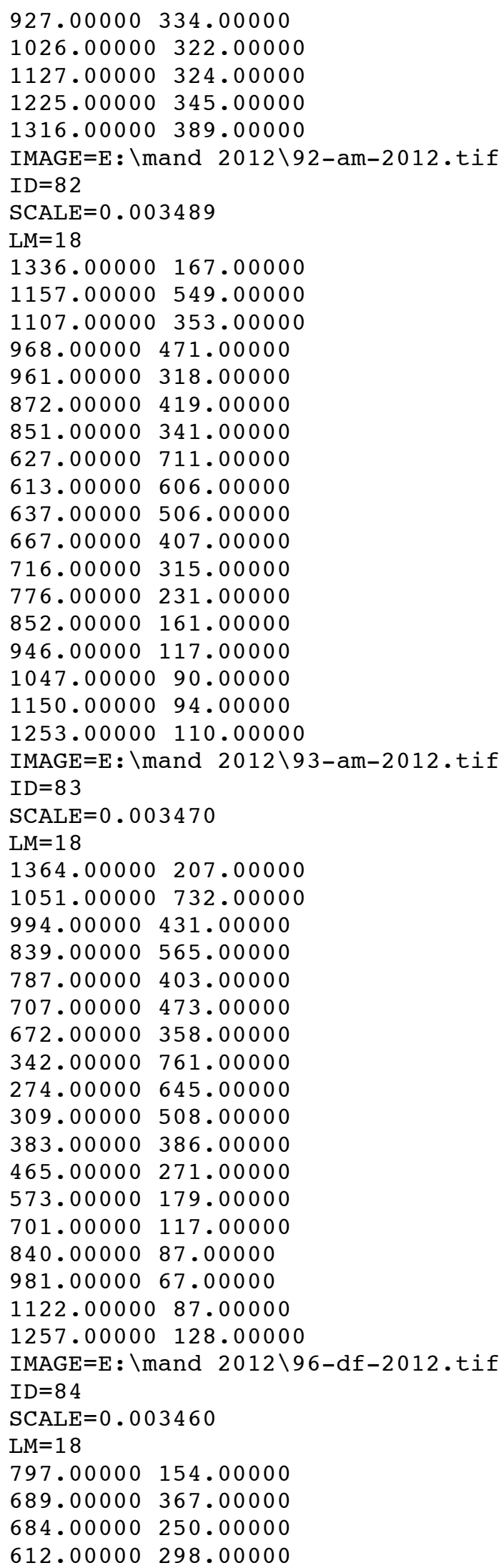




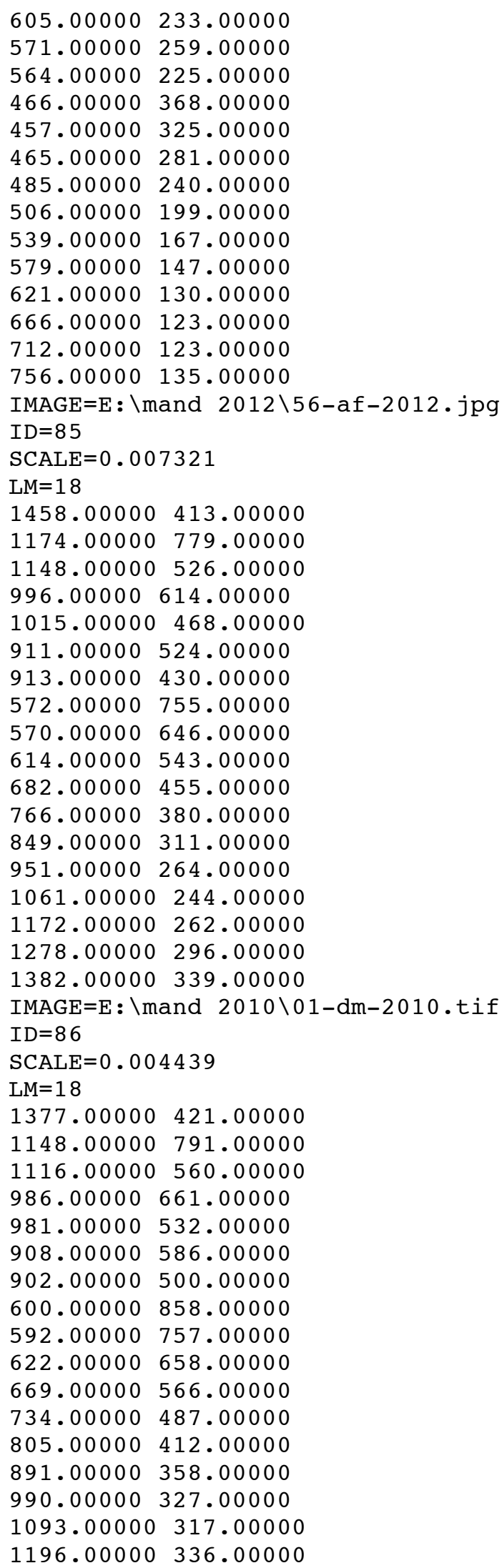




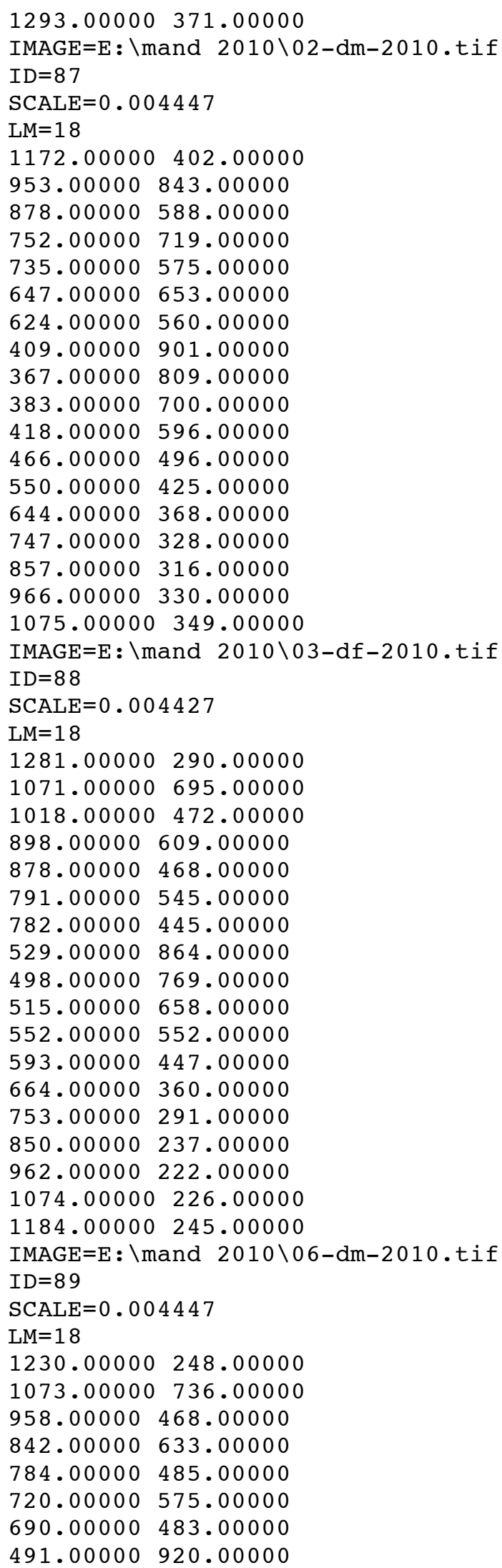




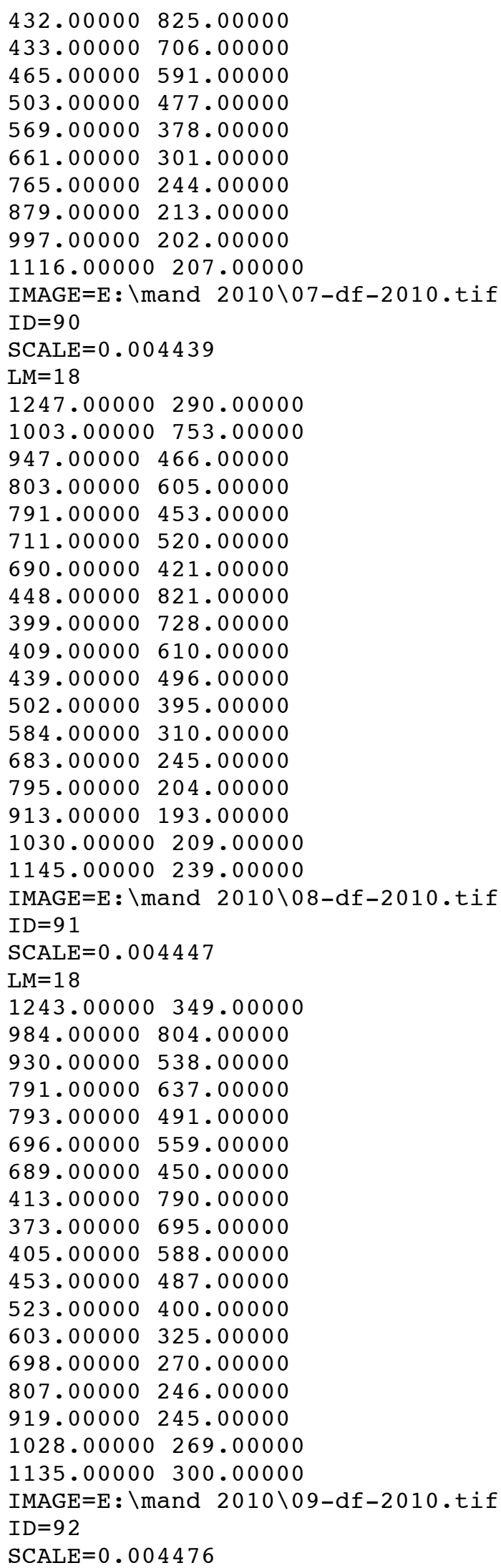




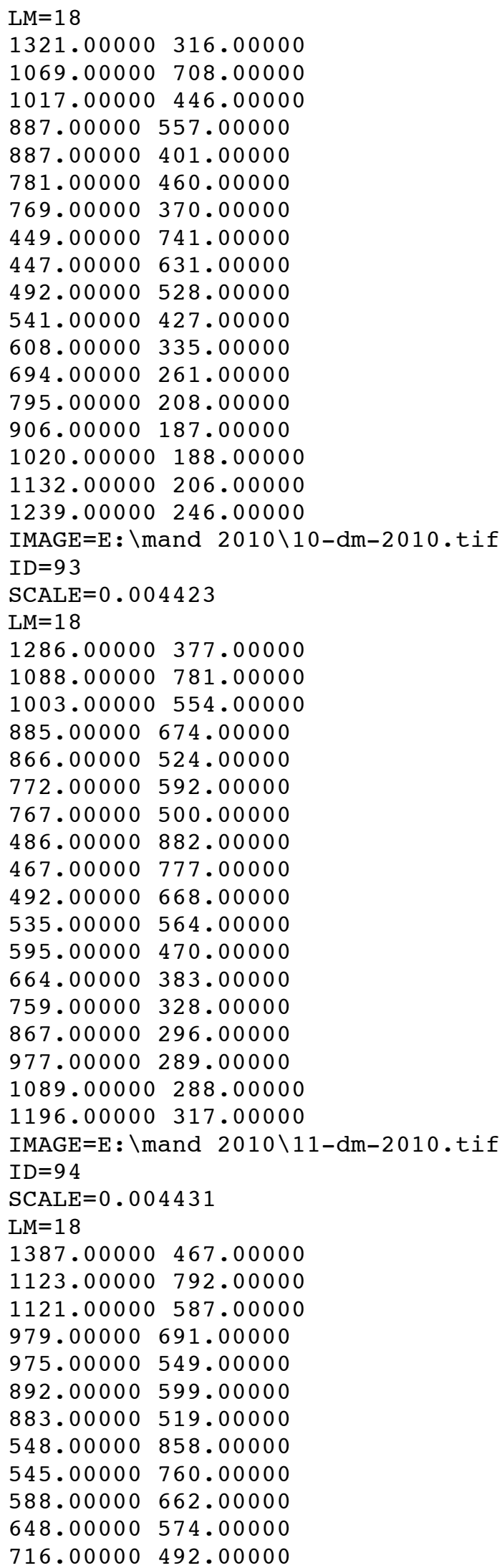




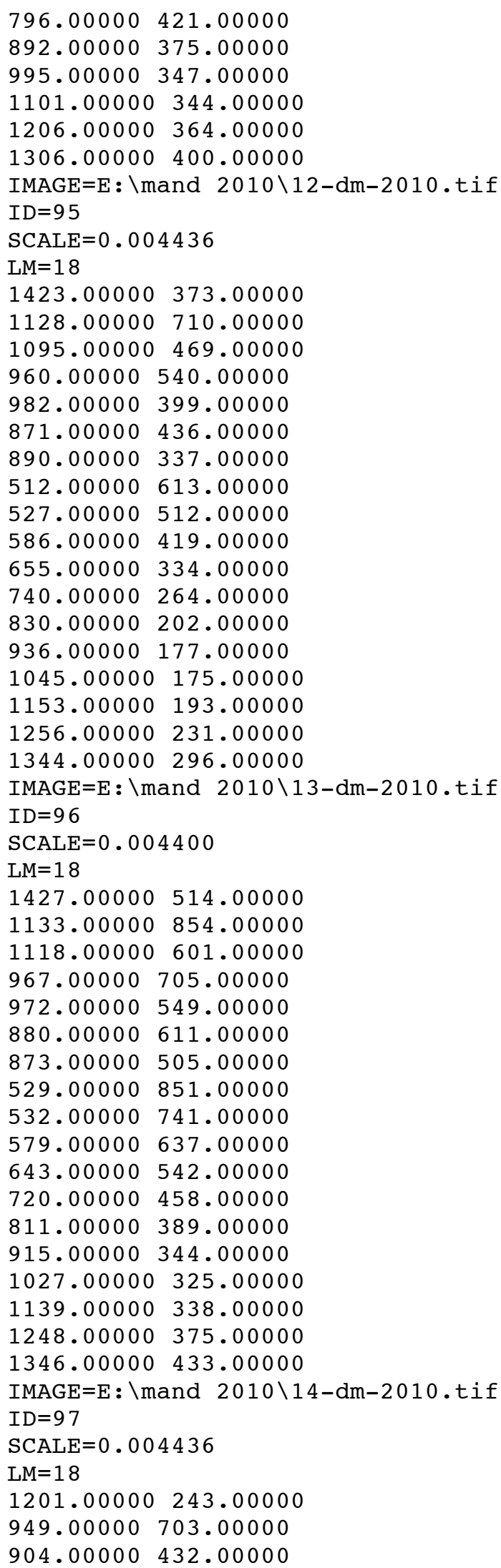




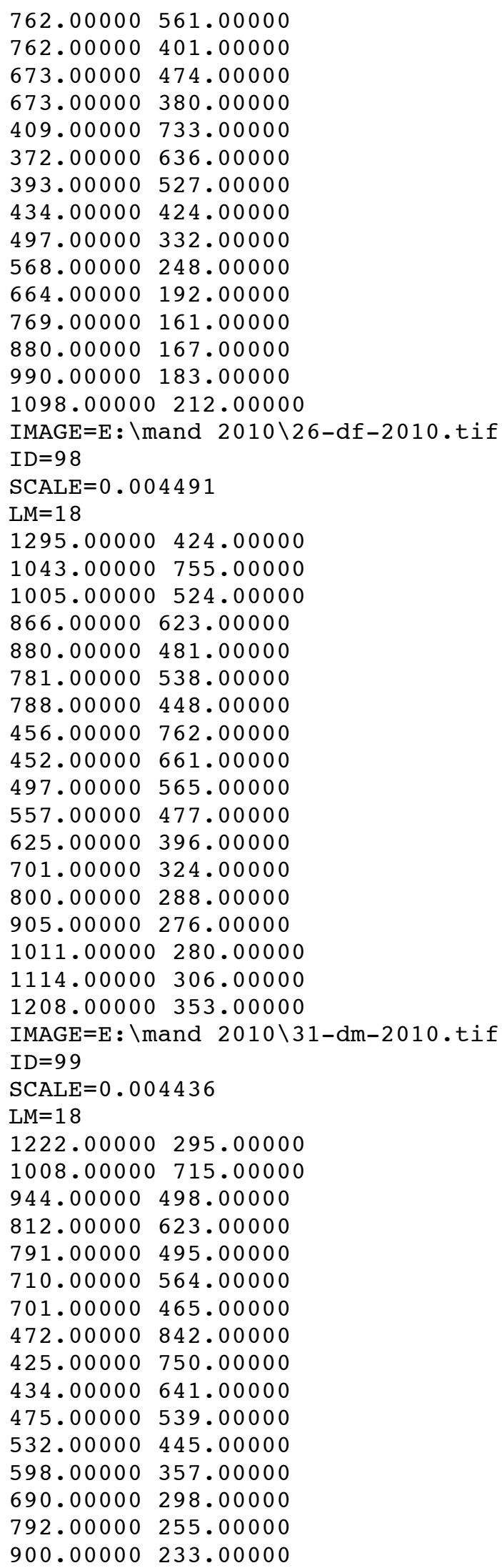




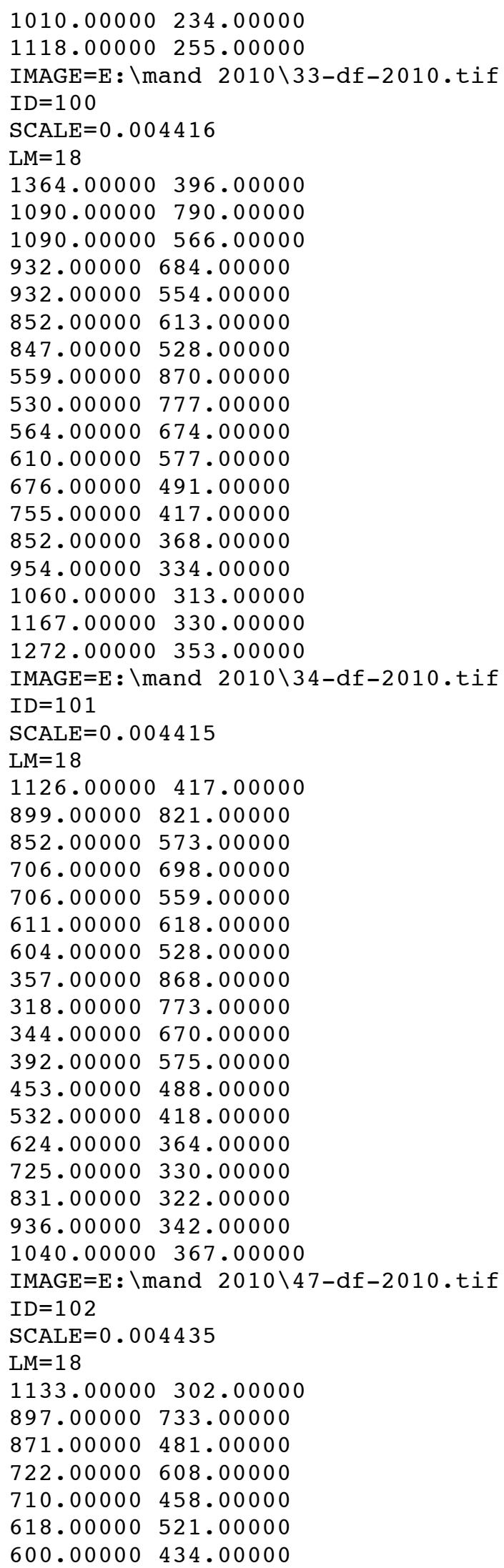




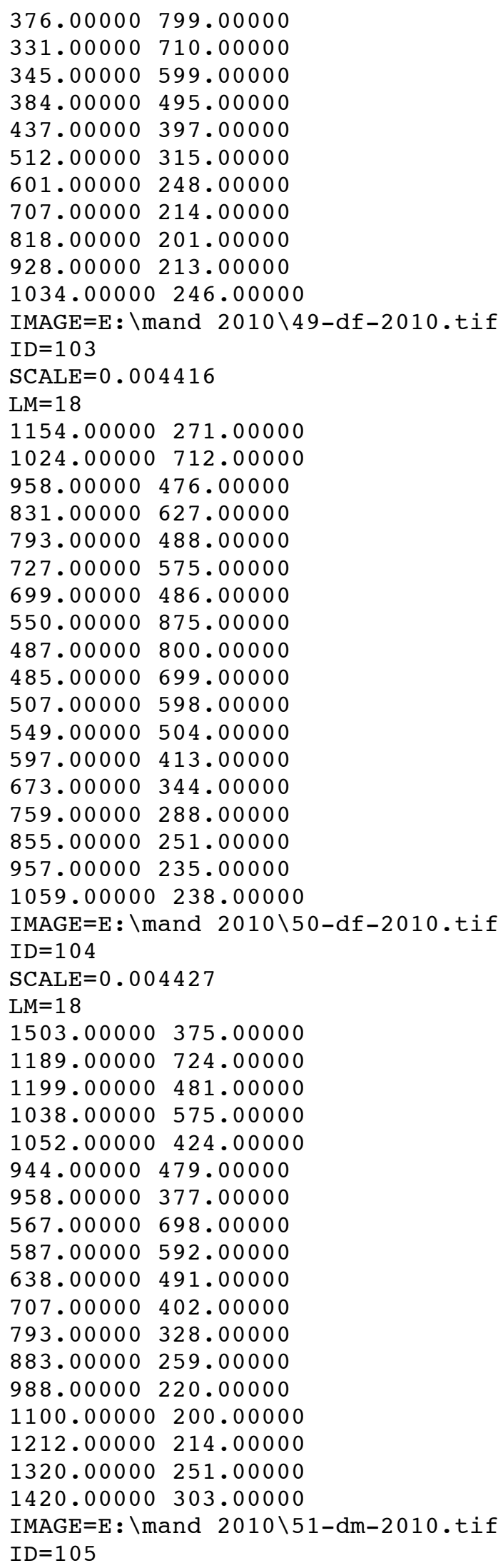




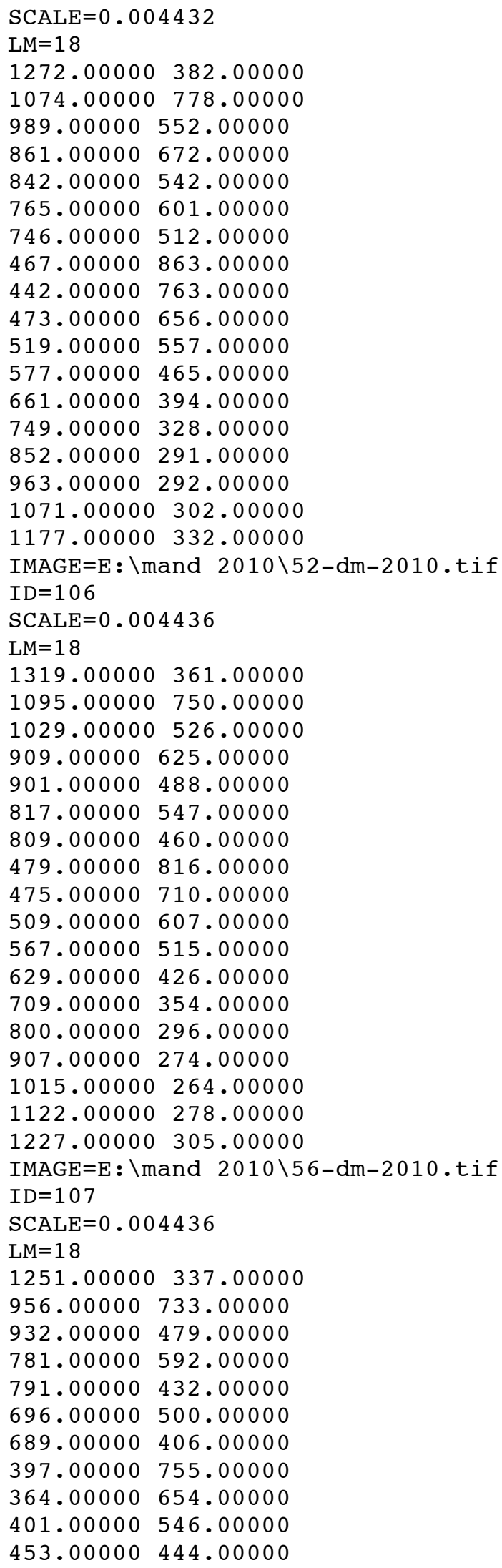




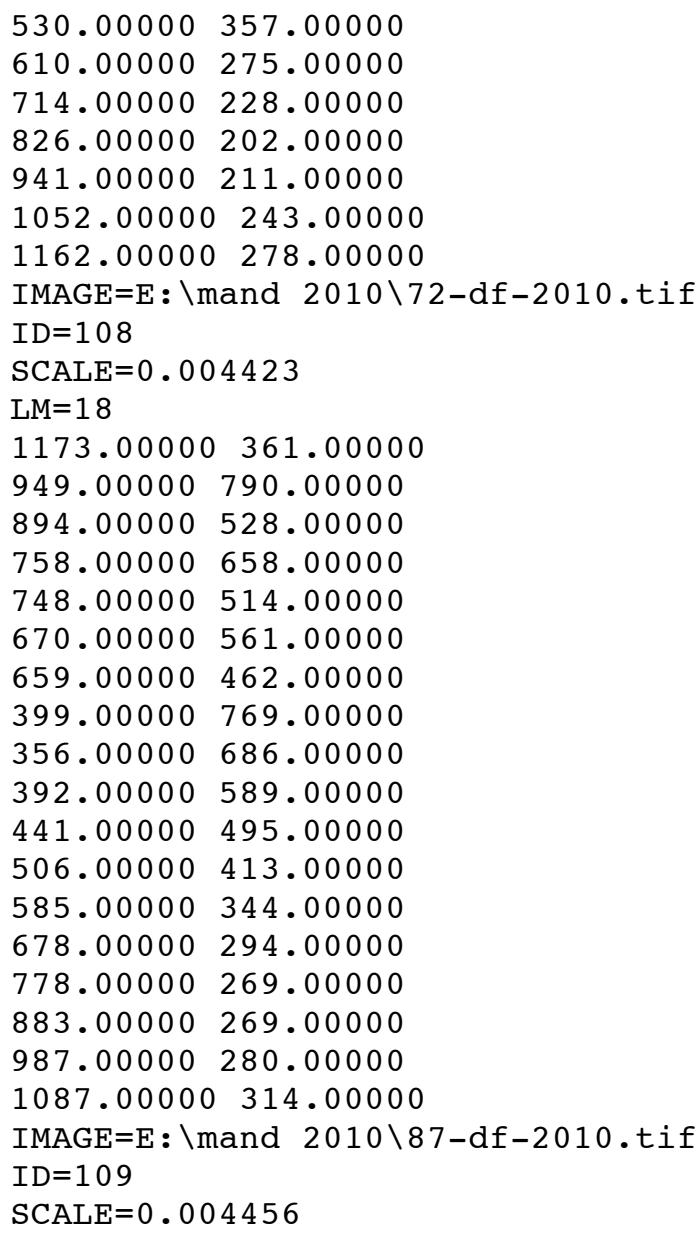

B.2) NTS format "sliders" file indicating sliding or "semi-"landmarks for the tps file given above.

" Sliders file

$\begin{array}{llll}1 & 10 & 3 & 0\end{array}$

$899 \quad 10$

$9 \quad 10 \quad 11$

$\begin{array}{lll}10 & 11 & 12\end{array}$

$\begin{array}{lll}11 & 12 & 13\end{array}$

$\begin{array}{lll}12 & 13 & 14\end{array}$

$13 \quad 14 \quad 15$

$\begin{array}{lll}14 & 15 & 16\end{array}$

$\begin{array}{lll}15 & 16 & 17\end{array}$

$\begin{array}{lll}16 & 17 & 18\end{array}$

$\begin{array}{lll}1 & 18 & 17\end{array}$ 
Appendix C: Data used for mating duration analyses in CSV file format.

A number of variables were included in the datafiles but were not used for analysis due to subsequent absence of meaningful or relevant trends or changes in experimental design.

C.1) Data for effects of time of initiation and baseline mating duration experiments (2011). $\mathrm{F}=$ individual female ID, $\mathrm{M}=$ individual male ID, $\mathrm{SP}=$ species $(\mathrm{a}=$ O. audouini, $\mathrm{d}=O$. dejeanii). All other headers are variables defined in Table 4.1 and text of Chapter 4.

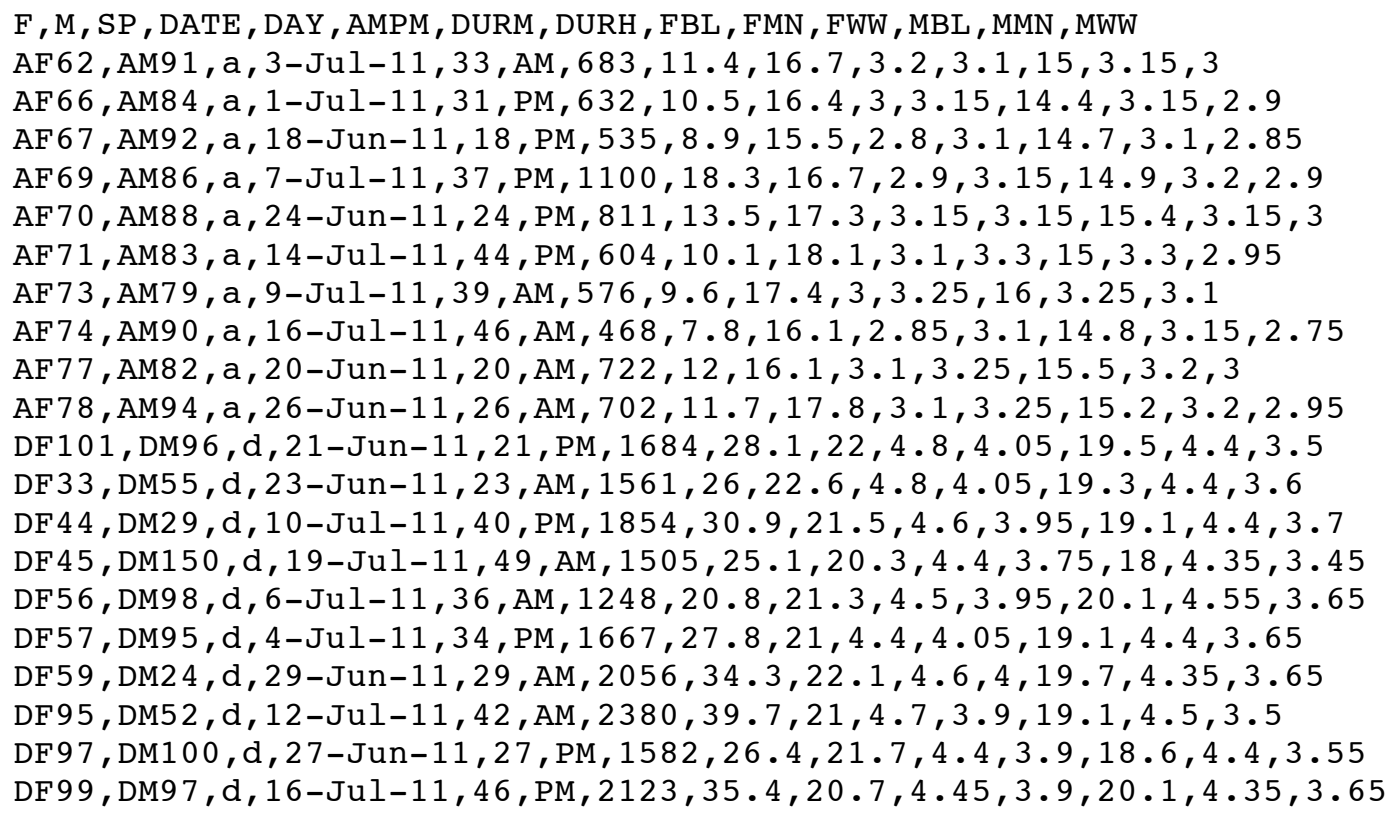

C.2) File name and associated file number (for use with Appendix C.3 below)

FILE \# FILE

$1 \quad 20120711-153011$

20120712-154133

20120806-143111

20120725-140810

20120725-140810

20120719-124128

20120722-144858

$20120712-154133$

$20120727-154707$

20120711-153011

20120727-154707

20120719-124128

20120722-144858

20120723-151431

20120802-120647

20120801-093305

20120801-093305

$20120802-120647$

20120804-140413 
$20120730-110139$

$20120709-140355$

$20120709-140355$

20120716-141416

$20120716-141416$

$20120730-110139$

C.3) Data used for effects of food deprivation on mating duration with morphometric data (2012). FLCD = file code (file number as given in Appendix C.2 above). All other headers are defined in text of Chapter 4.

FLCD , SP , FID , MID , Fst , Mst , DURM , DURH , FAGE , FACC , FPEL , FHW , FPW , FPL , FWW , FEW , FE $\mathrm{L}, \mathrm{FMN}, \mathrm{MAGE}, \mathrm{MACC}, \mathrm{MPEL}, \mathrm{MHW}, \mathrm{MPW}, \mathrm{MPL}, \mathrm{MWW}, \mathrm{MEW}, \mathrm{MEL}, \mathrm{MMN}, \mathrm{AED}$

$1, A, 53,9,48,1,637.7,10.6,41,7,12.7,3.95,4.3,3.6,3.1,5.85,9.1,2.78,57,23$ $, 12.7,3.8,4.05,3.5,3.05,5.5,9.2,3.2,6.004$

$2, A, 49,12,24,1,941.4,15.7,42,8,12.95,4,4.4,3.7,3.15,5.95,9.25,2.79,57,2$ $3,12.7,3.8,4.1,3.65,3.1,5.5,9.05,3.05,6.117$

$3, \mathrm{~A}, 35,13,1,1,712.4,11.9,36,7,12.95,4,4.45,3.75,3.2,5.8,9.2,2.72,49,20$, $12.2,3.6,3.95,3.45,2.85,5.6,8.75,3.1,5.979$

$4, \mathrm{~A}, 61,18,24,48,712,11.9,55,21,13,3.85,4.3,3.5,3.15,6.1,9.5,2.73,63,9,1$ $1.75,3.6,3.85,3.45,2.85,5.1,8.3,2.93,5.677$

$5, \mathrm{~A}, 55,25,24,48,494.6,8.2,55,21,13.1,3.85,4.35,3.65,3.2,6.15,9.45,2.77$, $49,13,12.15,3.6,4.1,3.55,2.95,5.45,8.6,3.11,6.047$

$6, \mathrm{~A}, 45,33,48,24,469,7.8,49,15,13.05,4,4.45,3.45,3.2,6.2,9.6,2.68,42,8,1$ $2.1,3.65,4,3.6,2.9,5.45,8.5,3.16,6.016$

$7, \mathrm{~A}, 60,40,48,48,628.2,10.5,52,18,12.65,3.75,4.2,3.5,3.15,5.8,9.15,2.69$, $53,17,12.3,3.75,4,3.55,3,5.35,8.75,3.04,0$

$8, \mathrm{~A}, 20,46,1,24,526.9,8.8,42,13,12.9,3.95,4.3,3.65,3.1,5.95,9.25,2.67,55$ $, 26,12.35,3.75,4.2,3.7,3.1,5.55,8.65,3.17,6.007$

$9, \mathrm{~A}, 62,48,1,24,748.6,12.5,57,23,13.1,3.95,4.45,3.5,3.15,6.1,9.6,2.85,62$ $, 8,11.8,3.45,3.7,3.45,2.85,5.35,8.35,3,5.76$

$10, A, 14,54,1,48,510.3,8.5,41,14,12.75,3.85,4.25,3.55,3.15,6,9.2,2.73,67$ $, 40,11.55,3.5,3.7,3.5,2.85,4.95,8.05,2.97,5.703$

$11, A, 51,65,48,1,770.3,12.8,57,23,13,3.9,4.25,3.75,3.15,6.15,9.25,2.7,41$

$, 7,11.9,3.5,3.85,3.45,2.85,5.2,8.45,3.1,5.818$

$12, A, 64,77,48,24,706,11.8,49,15,13.25,3.95,4.5,3.75,3.25,6.15,9.5,2.79$,

$62,38,12.45,3.75,4.15,3.6,3.1,5.65,8.85,3.19,5.776$

$13, \mathrm{~A}, 8,78,48,48,727.8,12.1,52,25,12.15,3.8,4.15,3.45,3,5.6,8.7,2.71,42$, $15,12.1,3.7,3.95,3.55,2.95,5.5,8.55,3.22,5.83$

$14, \mathrm{~A}, 3,80,1,1,751.4,12.5,53,26,13.25,4,4.45,3.75,3.35,6.15,9.5,2.69,41$, $14,12.05,3.65,4,3.55,2.95,5.3,8.5,3.09,5.755$

$15, \mathrm{~A}, 56,82,24,24,350.2,5.8,63,29,12.85,4.05,4.4,3.75,3.2,6.15,9.1,2.86$, $52,16,11.15,3.3,3.65,3.3,2.65,4.85,7.85,2.9,5.404$

$16, \mathrm{~A}, 69,84,24,1,495.6,8.3,62,28,13.05,3.8,4.35,3.6,3.1,5.95,9.45,2.71,6$ $3,9,12.1,3.7,4,3.55,2.95,5.4,8.55,3.27,5.997$

$17, \mathrm{~A}, 30,87,1,48,502.5,8.4,62,32,13.1,3.8,4.3,3.65,3.1,5.95,9.45,2.8,55$, $26,12.4,3.6,4,3.55,3,5.4,8.85,3.11,5.971$

$18, \mathrm{~A}, 36,89,24,24,305.4,5.1,63,34,11.5,3.65,4.05,3.5,2.95,5.75,8,2.56,52$ $, 23,11.95,3.6,3.85,3.45,2.9,5.3,8.5,3.12,5.961$

$19, \mathrm{D}, 24,21,24,24,1044.9,17.4,65,36,15.95,5.2,6.5,4.4,3.75,7.8,11.55,4.1$ $4,65,36,13.8,4.6,5.5,3.95,3.3,6.75,9.85,3.93,7.068$

$20, \mathrm{D}, 101,26,1,1,1658,27.6,60,5,15.85,5.3,6.6,4.5,4,8.1,11.35,4.01,60,31$ $, 15.1,4.9,6.05,4.5,3.65,7.5,10.6,4.27,7.903$

$21, \mathrm{D}, 42,27,24,1,2153.6,35.9,39,5,16.7,5.55,7.05,4.75,4.2,8.2,11.95,4.49$

$, 39,10,13.9,4.5,5.5,4.05,3.3,6.85,9.85,4.18,0$ 
$22, \mathrm{D}, 70,34,1,24,2409.5,40.2,39,3,16.5,5.55,6.7,4.7,3.8,8.2,11.8,4.34,39$ $, 10,14.85,4.85,5.85,4.2,3.5,7.15,10.65,4.32,7.939$

$23, \mathrm{D}, 19,72,48,1,1693.1,28.2,46,17,16.25,5.4,6.65,4.7,4,8.45,11.55,4.42$, $46,10,15.05,4.7,5.9,4.5,3.45,7.2,10.55,4.21,7.722$

$24, \mathrm{D}, 75,74,1,48,1725.6,28.8,46,10,16.05,5.5,6.85,4.55,4,8.25,11.5,4.56$, $46,10,14.65,4.65,5.8,4.15,3.55,7.1,10.5,4.15,7.652$

$25, \mathrm{D}, 96,76,48,48,1301.8,21.7,60,6,16,5.45,6.6,4.65,4,8.1,11.35,4.15,60$, $24,13.85,4.65,5.55,4.15,3.4,6.7,9.7,3.97,7.543$

C.4) Data used for comparing effects of operational sex ratio and food deprivation on mating duration (2012). File $=$ file name, $\mathrm{EXP}=$ experiment type $(\mathrm{osr}=$ operational sex ratio, feed $=$ food deprivation), Type $=$ type of experiment (explained in text of Chapter 4), $\mathrm{SP}=$ species $(\mathrm{a}=O$. audouini, $\mathrm{d}=O$. dejeanii $)$, Bottom $=$ individual specimen ID for mounted beetle, Top $=$ individual specimen ID for mounting beetle, MAX $=$ longest single mounting event (in minutes), TOTAL = total time spent in mount for pair for duration of recording period (in minutes).

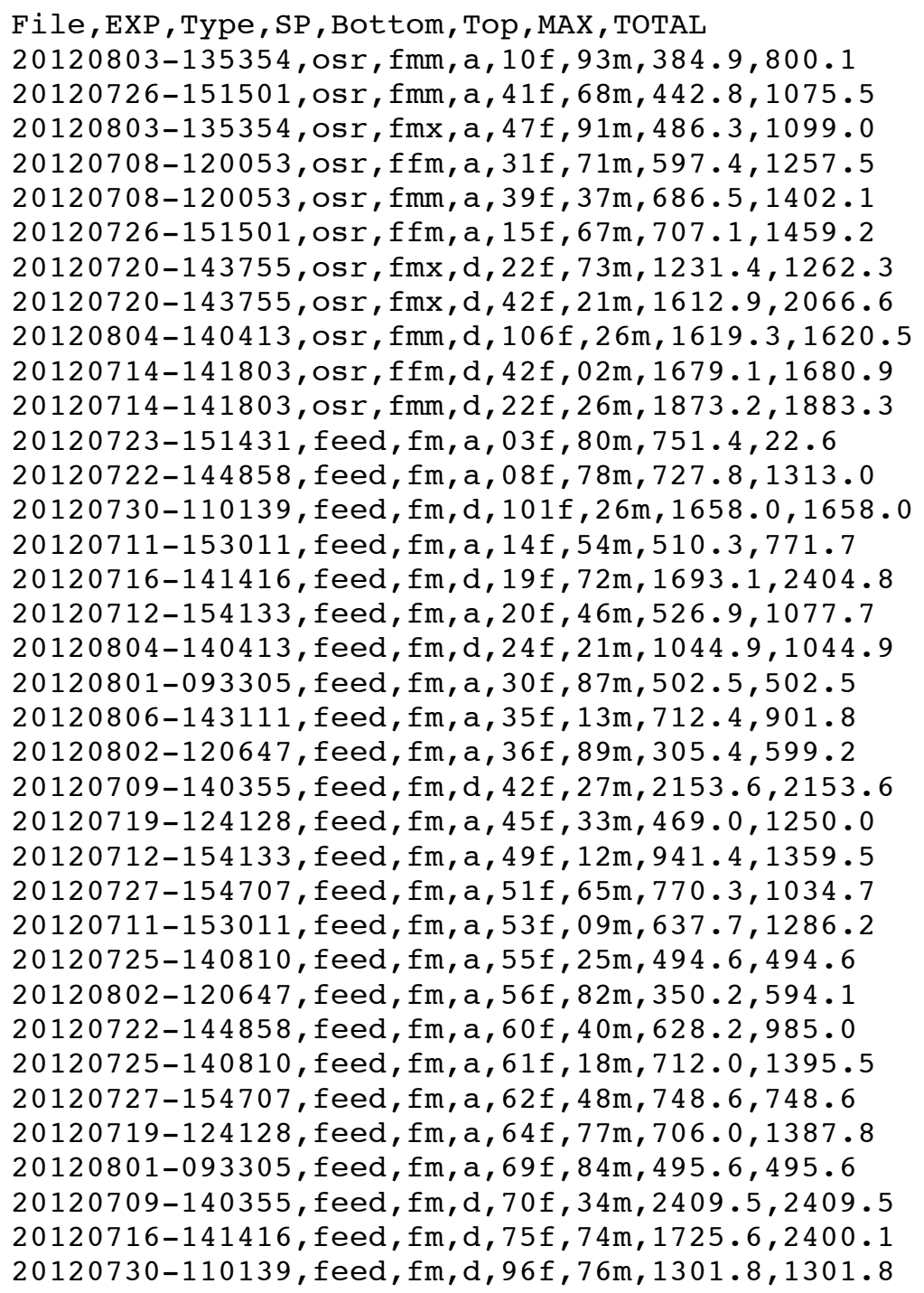




\section{Appendix D: Laboratory husbandry of wild-caught Omus audouini and O. dejeanii}

Beetles were maintained individually in commercially available food-grade containers purchased from a local restaurant supply retailer. For 2011, Omus dejeanii were held in $12 \mathrm{oz}$ lidded "deli-style" containers while O. audouini were held in $9 \mathrm{oz}$ drinking cups covered with dome lids with $c a .2-3 \mathrm{~cm}$ of native soil as a substrate and a surface cover of native forest floor litter (predominately scales of Douglas-fir cones, leaf fragments and fir needles). Prior experiences found that the tarsal pads of male $O$. audouini allowed them to adhere to and climb-up smooth polyethylene. Dome lids with wide holes were found to be adequate covers; the males were unable to support themselves along the underside of the lid while the wide hole allowed for suitable ventilation. This was not an issue for $O$. dejeanii due to their larger body mass; they were unable to support themselves along the vertical surface but the tarsal pads were observed to cling to the surface of the walls of the enclosure.

It was found that male tarsal pads of Omus do not adhere well to either polypropylene or polystyrene containers making these preferred materials for enclosures for the aforementioned reason in addition to their availability, low-cost and durability. For 2012, both species were maintained individually in 12 oz polypropylene containers with a substrate of $c a 2-3 \mathrm{~cm}$ of native soil, enough forest litter (mostly leaf and bark fragments) to lightly cover the bare soil surface and a loosely fitted lid.

Beetles were maintained on a 13/9h light/dark cycle approximately matching the natural seasonal light cycle with a mean ambient high/low temperature cycle of $24 / 21^{\circ} \mathrm{C}$. Captive Omus were fed ad libitum five to six times a week on organic, hormone-free low fat ground beef (5-10\%), which was most often readily accepted when either directly presented via forceps or when left on the substrate. The diet was supplemented weekly with a small piece $\left(\mathrm{ca} 0.3 \mathrm{~cm}^{3}\right)$ of commercial spirulina-based cricket food under the assumption that wild caught prey would be "gut-loaded" and provide a certain degree of plant-based nutritional supplements. These supplements were often at least partially, if not mostly, ingested but it is uncertain whether they where consumed due to perceived nutritional content or moisture content. Uneaten food was removed prior to offering new food to reduce the growth of fungus and bacteria. Humidity and ingestible water was 
maintained by regular misting with distilled water and the addition of $2-3 \mathrm{~cm}^{3}$ of polyacrylamide gel to each enclosure and replenished or replaced as needed.

Handling of the beetles was kept to a minimum. If being transferred between enclosures, the beetles were gently motivated to self-ambulate into a small transfer enclosure (a small, plastic 2 oz cup with steep sides). Occasionally an instrument (spoon or forceps) would be needed to gently probe and direct the movement of the individual into the transfer cup. The temperament of individual beetles was found to differ, with some regularly attacking foreign objects while other individuals had a regular response to run and or hide in the substrate.

Occasionally, it was necessary to cause a beetle to release its bite, either from an object, another beetle or, on rare occasions, the finger of the researcher. It was found that these species of Omus will generally bite with vigor and persistence and do not readily release their grip. Forcing the beetle to disengage would likely result in mandibular disarticulation and probably death of the specimen. It was found that gently spraying the beetle with a misting bottle of distilled water was the most reliable method to cause individuals to self-disengage while avoiding undue injury. 


\section{Appendix E: Microdissection of Omus aedagus}

While the processes involved in the measuring of external anatomy of animals is often self-evident, I found frusteratingly few resources to guide me on the extraction of internal structures. While male tiger beetle anatomy has been well described (Horn 1930, Freitag et al. 1980), the process of removing the genitalia are rarely given in detail and, furthermore, the images given are disembodied and without positional context. As a consequence I was motivated to develop an explicit protocal governing the process of male genitalia extraction starting from either a freshly killed or EtOH-preserved specimen to fully disembodied organ.

Specimen: \#34, Omus dejeanii (male)

Collected: 29 June 2012, Powell Butte Nature Park, Portland, Oregon.

Collection method: Dry pitfall trap

Specimen storage: $70 \%$ ethanol (pre- and post-dissection)

Dissected: 16 Aug 2012 by R.K. Richardson (Ruedas Lab, Biology Department, Portland State University)

All images taken by R. K. Richardson.

The following protocols apply to both Omus dejeanii and O. audouini. The specimen of $O$. dejeanii was used for this document due to its greater size and, hence, greater visibility. These protocols may be general enough to apply to general carabid beetle aedagus dissection/extraction.

Materials: Iris/cuticle scissors, forceps, entomology pins/fine-tipped probes, dissecting microscope, fine scalpel (e.g. \#11; optional) 


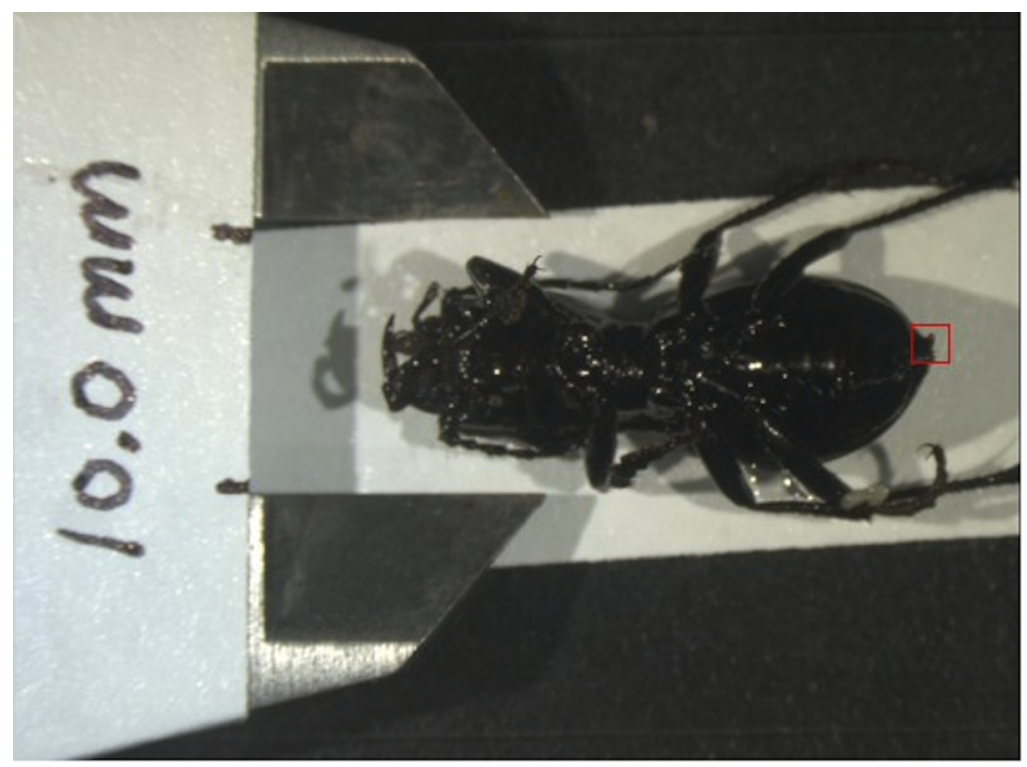

Figure E.1: Male Omus dejeanii, ventral side up. Distal end of aedagus highlighted by box (aedague $=$ male intromittent organ, technically penis and parameres together).

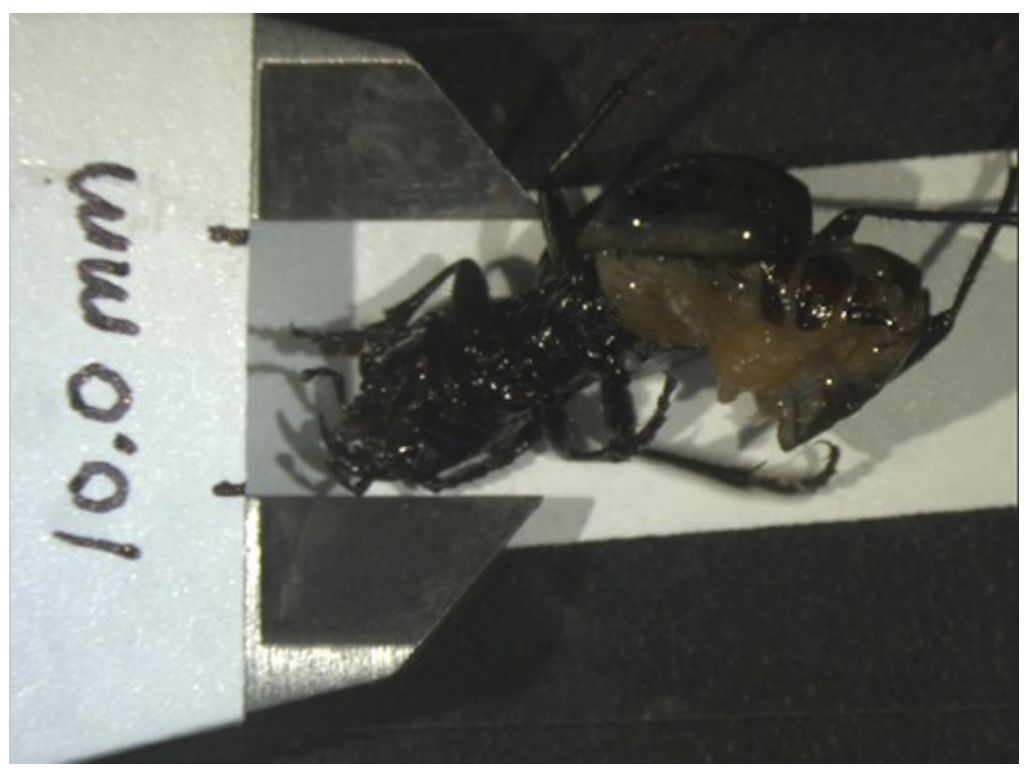

Figure E.2: Abdomen is carefully cut along the lateral edges using iris/cuticle scissors 


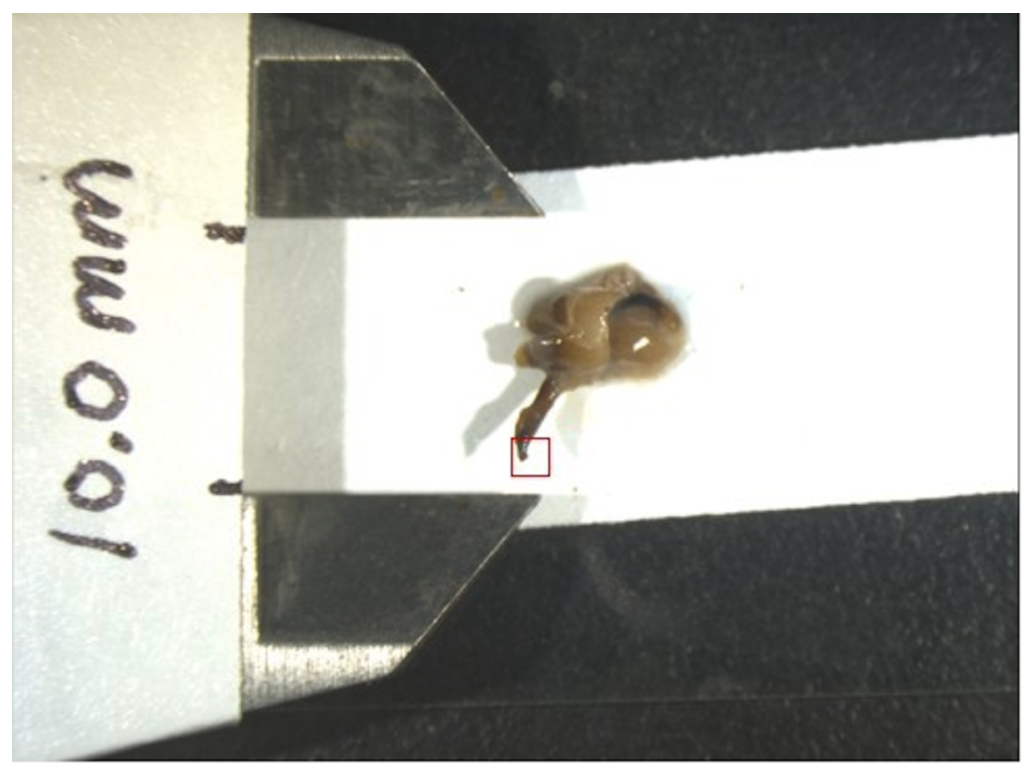

Figure E.3: Male organs and associated visceral tissues. Distal tip of aedagus highlighted by box for positional context. Use entomology pins/needle probe and scissors to separate tissues. There is very little need for any actual cutting - instead use blunt dissection methods to separate tissues.

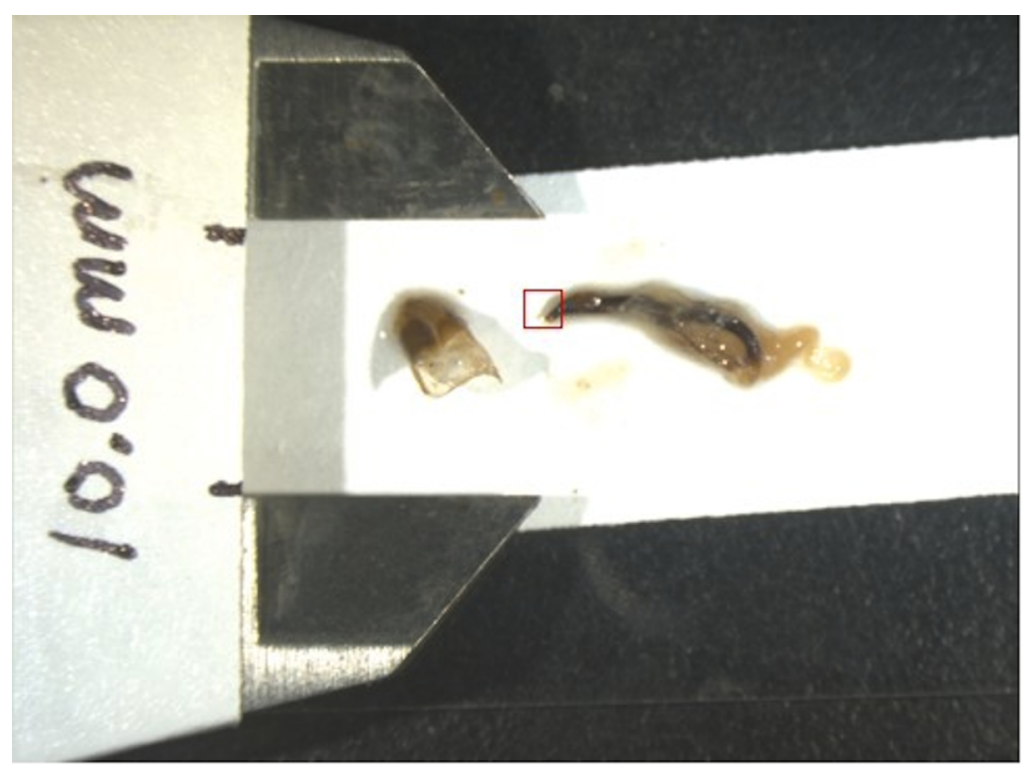

Figure E.4: Aedagus shown in connective tissue sheath. Distal tip highlighted by box for positional context. Use scissors to carefully cut along the sheath and remove aedagus. Stabilize with pins as needed. 


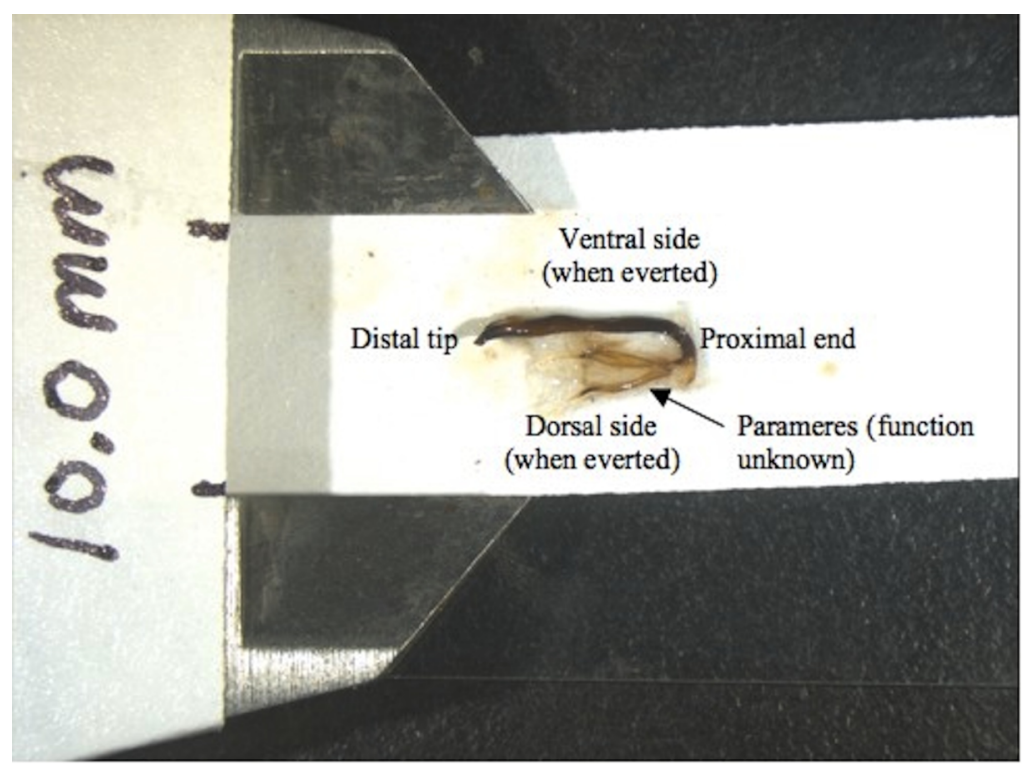

Figure E.5: Fully extracted and cleaned aedagus. 